\title{
A Case-Based Assistant for Diagnosis and Analysis of Dysmorphic Syndromes
}

\author{
Carl David Evans
}
A dissertation submitted in partial fulfillment
of the requirements for the degree of
Doctor of Philosophy
of the
University of London

Department of Computer Science
University College London

1994 
ProQuest Number: 10017424

All rights reserved

INFORMATION TO ALL USERS

The quality of this reproduction is dependent upon the quality of the copy submitted.

In the unlikely event that the author did not send a complete manuscript and there are missing pages, these will be noted. Also, if material had to be removed, a note will indicate the deletion.

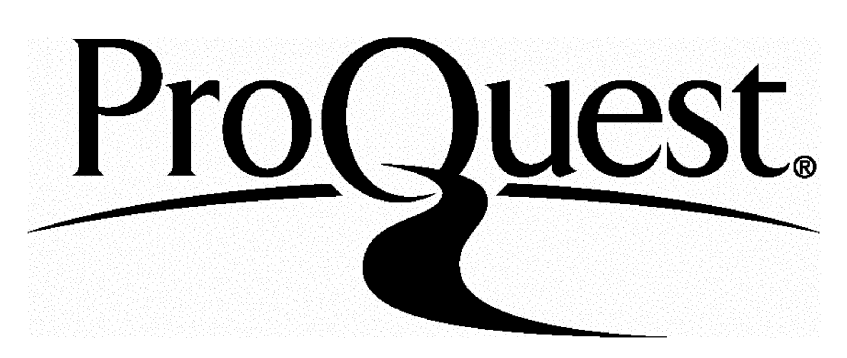

ProQuest 10017424

Published by ProQuest LLC(2016). Copyright of the Dissertation is held by the Author.

All rights reserved.

This work is protected against unauthorized copying under Title 17, United States Code. Microform Edition (c) ProQuest LLC.

\author{
ProQuest LLC \\ 789 East Eisenhower Parkway \\ P.O. Box 1346 \\ Ann Arbor, MI 48106-1346
}




\section{Abstract}

Dysmorphology is a field of medicine which has as one of its concerns the diagnosis of children born with multiple malformations. A pattern of malformations recognised as occurring together and thought to be pathogenetically related is collectively called a dysmorphic 'syndrome'. Abnormalities (dysmorphic features) may pertain to clinical, radiological, biochemical, histological or chromosomal defects. This diversity is reflected in the source of diagnostic expertise, which is relatively sparse and is provided by specialists from varying disciplines such as clinical medicine, genetics and radiology.

Approximately 8 in 1000 of children are born with multiple malformations, and about half of these infants will be linked with a chromosomal disorder (diagnosed by performing a karyotype). Of the rest, diagnosis (to recognised syndromes) is more difficult and is a task performed by experienced specialists. Diagnosis is not always possible. About forty per cent of cases remain undiagnosed with respect to known disorders, and recognition of new syndromes is an important facet of dysmorphology. To assist such investigation, the physician has at hand reference sources such as journals, syndrome compendia, and more recently, computer databases. Whilst databases may assist diagnosis, the functions performed by specialists that invovle learning new syndromes have not been automated to any degree. This aspect provides the focus for this research.

The diagnosis and learning tasks of dysmorphology map intuitively with ideas from artificial intelligence: case-based reasoning (CBR) and learning (CBL). The thesis reports on the utilisation of a case-based approach in order to develop a diagnostic aid with an explicit goal of automating the learning aspect of dysmorphology. The initial focus of the research concerns the development of a case-based learning algorithm which simulates the learning processes that exist in dysmorphology, and which provides the basis for a dynamic case-based architecture. The thesis proceeds in view of relevant problems and issues highlighted by these experiments when viewed in the context of developing a realistic model for a CBR system within dysmorphology. This includes an investigation of a model for similarity assessment and the interdependent design issues of a case representation and case memory. An interactive CBR model is proposed that by default assists in diagnosis through CBR, but further extends the scope of syndrome database through its learning capability. 


\section{Acknowledgements}

I would like to thank a number of people at the department of computer science at UCL. I am particularly grateful to Elpida Keravnou and John Washbrook for their guidance and support throughout the period of this research. Also, I would also like to express thanks to Derek Long, Felicity Dams and Michael Luck for their invaluable assistance whilst preparing this thesis.

This research would not have been possible without the help a number of medical specialists who have found the time to assist me in my work. For this I am very grateful to Dr. Christine Hall ${ }^{1}$, Dr. J. A. Maat-Kient ${ }^{2}$, Professor David L. Rimoin ${ }^{3}$, and Professor Robin Winter ${ }^{4}$.

This research has been supported by funding from the Science and Engineering Research Council.

\footnotetext{
${ }^{1}$ Great Ormond Street Hospital, London.

${ }^{2}$ On temporary visit to the Institute of Child Health, London.

${ }^{3}$ Cedars-Sinai Medical Center, UCLA.

Institute of Child Health, London.
} 


\section{Contents}

1 Introduction to Dysmorphology 14

1.1 Thesis Overview . . . . . . . . . . . . . . . . . 14

1.2 An Introduction to Dysmorphology . . . . . . . . . . . . . . . 16

1.3 Down Syndrome (Trisomy 21 Syndrome) . . . . . . . . . . . . . . 19

1.3.1 Diagnosticity and Occasional Features . . . . . . . . . . 21

1.3.2 Additional Diagnostic Information . . . . . . . . . . . 23

1.3.3 Etiology, Prognosis and Genetic Counseling . . . . . . . . . . . 24

1.4 Performance Tasks in Dysmorphology . . . . . . . . . . . 25

1.4 .1 Diagnosis . . . . . . . . . . . . . . . . 25

1.4 .2 Research . . . . . . . . . . . . . . . . . . 29

1.5 Conclusions and Goals of the Research . . . . . . . . . . . . . . . 31

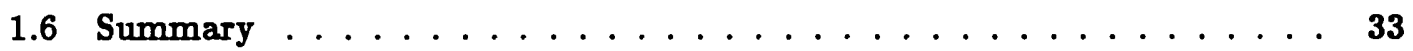

2 Dysmorphology, Computer Systems and AI 35

2.1 Introduction . . . . . . . . . . . . . . . . . . 35

2.2 The London Dysmorphology Database . . . . . . . . . . . . 36

2.2.1 LDDB System Goals . . . . . . . . . . . . . . . 37

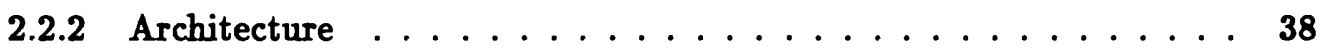

2.2.3 Mode of Operation . . . . . . . . . . . . . . 39

2.2 .4 Discussion . . . . . . . . . . . . . . . 42

2.3 The Skeletal Dysplasia Diagnostician . . . . . . . . . . . . . . 43

2.3.1 SDD System Goals . . . . . . . . . . . . . . . 43

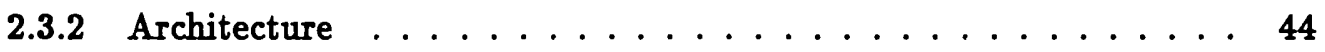

2.3.3 Mode of Operation . . . . . . . . . . . . . . . 46

2.3.4 Discussion . . . . . . . . . . . . . . . 48 
2.4 Related Work . . . . . . . . . . . . . . . . 50

2.4 .1 On-Line Databases . . . . . . . . . . . . . 50

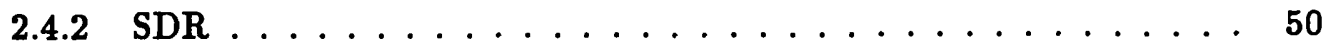

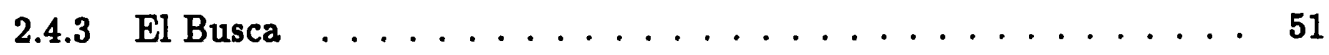

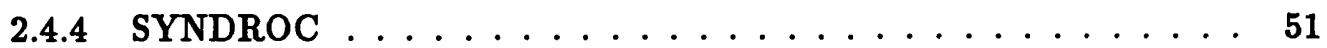

2.4 .5 Weiner . . . . . . . . . . . . . . . . 52

2.4 .6 GENDIAG $\ldots \ldots \ldots \ldots \ldots \ldots \ldots \ldots \ldots$

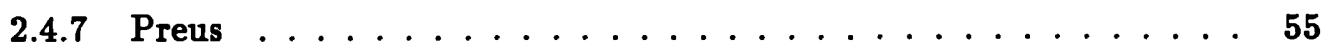

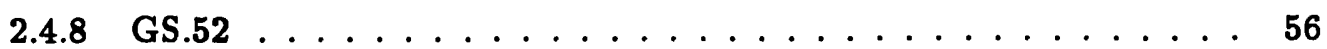

2.4 .9 DYSMOR ................... 57

2.4.10 Visual Reference Systems . . . . . . . . . . . 57

2.4 .11 Discussion . . . . . . . . . . . . . . 58

2.5 Modelling Procedures and Aspects of Dysmorphology . . . . . . . 60

2.6 Case-Based Reasoning and Learning . . . . . . . . . . . . 61

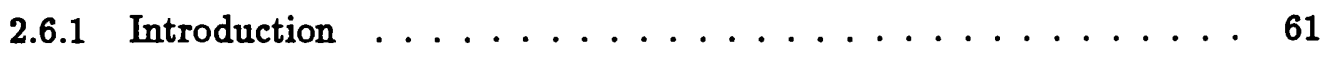

2.6.2 A General CBR Model . . . . . . . . . . . . . . . 6 63

2.7 Incremental Concept Formation . . . . . . . . . . . . . . . 66

2.8 Conclusions and Philosophy of Approach . . . . . . . . . . . 71

2.9 Summary . . . . . . . . . . . . . . . . . 74

3 Case Representation and Memory Organisation 75

3.1 Introduction . . . . . . . . . . . . . . 75

3.2 System Software . . . . . . . . . . . . . . 77

3.3 Case Representation $\ldots \ldots \ldots \ldots \ldots \ldots \ldots$

3.3.1 Case Structure . . . . . . . . . . . . . . 80

3.3.2 Case Generation and Storage . . . . . . . . . . 82

3.3.3 Generic Access and Utility Functions . . . . . . . . . . . . . 83

3.4 Memory Organisation and Indexing . . . . . . . . . . . . . . 85

3.4.1 Indexing in Distributed Case Memory: An Extended Example . . 87

3.4 .2 Discussion . . . . . . . . . . . . . . . . . . . 91

3.5 Case-Based Learning with Distributed Memory . . . . . . . . . . . . 92

3.6 Conclusions . . . . . . . . . . . . . . . . . . . 95

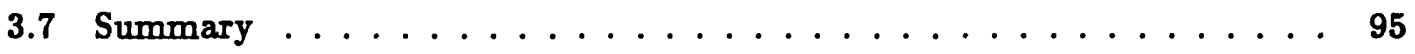


4 Experiments with a Case-Based Learning Algorithm 97

4.1 Introduction . . . . . . . . . . . . . . . . 97

4.2 Discrimination Networks . . . . . . . . . . . . . . . 98

4.3 The UNIMEM Algorithm . . . . . . . . . . . . . . . . . 101

4.4 Experiments with UNIMEM . . . . . . . . . . . . . . . . . . . 105

4.4.1 Acrocephalosyndactyly Data Set . . . . . . . . . . . . 105

4.4.2 Skeletal Dysplasias Data Set . . . . . . . . . . . . . 106

4.4.3 Chromosomal Abnormality Test Cases . . . . . . . . . . . . 106

4.4 .4 Results ....................... 110

4.5 Discussion . . . . . . . . . . . . . . . . . . . 122

4.6 A Critique of UNIMEM . . . . . . . . . . . . . . . . 125

4.7 Conclusions . . . . . . . . . . . . . . . . . 127

4.8 Summary . . . . . . . . . . . . . . . . . . 129

5 Integrating Domain Knowledge within the CBL Model 131

5.1 Introduction . . . . . . . . . . . . . . . . . . . . . . . 131

5.2 The LDDB Model of Diagnostic Significance . . . . . . . . . . . . . 132

5.3 The W-UNIMEM Algorithm . . . . . . . . . . . . . 133

5.4 Results . . . . . . . . . . . . . . . . . . 138

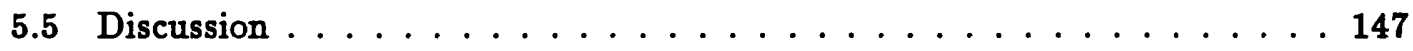

5.6 Conclusions . . . . . . . . . . . . . . . . . . . 149

5.7 Summary . . . . . . . . . . . . . . . . . 152

6 A General Theory of Similarity $\quad 154$

6.1 Introduction . . . . . . . . . . . . . . . . . 154

6.2 Tversky's Theory of Similarity . . . . . . . . . . . . . 155

6.2.1 The Contrast Model . . . . . . . . . . . . . . . . 157

6.2 .2 Asymmetry and Focus . . . . . . . . . . . . . 157

6.2 .3 Similarity versus Difference . . . . . . . . . . . . 158

6.2.4 Similarity in Context . . . . . . . . . . . . . . . . 158

6.2.5 The Diagnosticity Principle . . . . . . . . . . . . . . . 159

6.2 .6 Discussion . . . . . . . . . . . . . . . . . 159

6.3 Integrating the Contrast Model within CBL . . . . . . . . . . . . . 162

6.3.1 The Extended Contrast Model . . . . . . . . . . . . . 163 
6.3.2 The C-UNIMEM Algorithm . . . . . . . . . . . . . . . 164

6.3 .3 Results ........................ 166

6.3.4 Discussion . . . . . . . . . . . . . . . . 175

6.4 A Critique of the CBL Model . . . . . . . . . . . . . 183

$6.5 \mathrm{CBR}$ and General Similarity Assessment . . . . . . . . . . . 187

6.6 Conclusions . . . . . . . . . . . . . . . . . . . 190

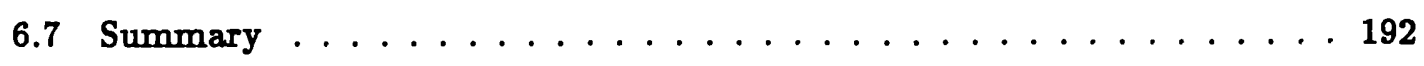

7 A CBR Assistant for Diagnosis and Analysis of Dysmorphic Syndromes 194

7.1 Introduction . . . . . . . . . . . . . . . . . . . 194

7.2 A CBR Assistant Model and Procedure . . . . . . . . . . . . . 197

7.3 Ranking Hypotheses with the Contrast Model . . . . . . . . . . . 199

7.3.1 Experiments with the Ranking Model . . . . . . . . . . . 201

7.3.2 Discussion . . . . . . . . . . . . . . . . . 204

7.4 Case-Based Learning with the Case-Based Assistant Model . . . . . . . 208

7.4 .1 Interactive CBL Procedure . . . . . . . . . . . . 210

7.4 .2 Discussion . . . . . . . . . . . . . . . 213

7.4 .3 Additional Functionality . . . . . . . . . . . . 215

7.5 Additional Utilities . . . . . . . . . . . . . . . . . 216

7.5.1 The Indexing Model . . . . . . . . . . . . . . . 216

7.5.2 Editing the Diagnostic Model . . . . . . . . . . . . 217

7.6 Conclusions . . . . . . . . . . . . . . . . 217

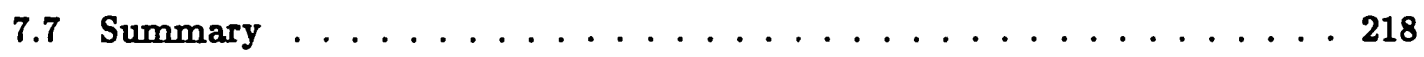

8 Conclusions and Future Work $\quad 220$

8.1 Introduction . . . . . . . . . . . . . . . . . 220

8.2 Relationship to other work . . . . . . . . . . . . . . 221

8.2.1 Computer Systems in Dysmorphology . . . . . . . . . . 221

$\mathbf{8 . 2 . 2}$ CBR Models in Medicine . . . . . . . . . . . . . 222

8.3 Contributions to Research . . . . . . . . . . . . . . . 224

8.3.1 Contribution to Dysmorphology . . . . . . . . . . . . 224

8.3.2 Contribution to Case-Based Reasoning . . . . . . . . . . . . 226

8.4 Critical Analysis $\ldots \ldots \ldots \ldots \ldots \ldots \ldots$

8.4 .1 Case-Based Learning . . . . . . . . . . . . . . 231 
8.4.2 The CBR Assistant Model . . . . . . . . . . . . . . . 232

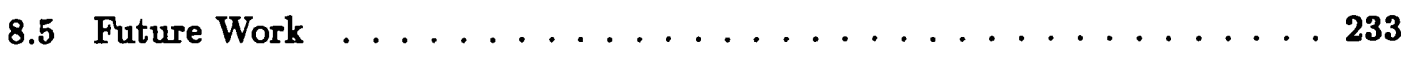

A Terms and Procedures in Dysmorphology 235

A.1 Terms of Anatomical Development . . . . . . . . . . . 235

A.1.1 Individual Alterations of Form or Structure . . . . . . . 235

A.1.2 General Terminology . . . . . . . . . . . . . . 237

A.1.3 Patterns of Morphologic Defects . . . . . . . . . . 237

A.2 General Principles of Dysmorphology . . . . . . . . . . . . . 239

A.2.1 Nonspecificity of Individual Defects . . . . . . . . . . 239

A.2.2 Variance in Expression . . . . . . . . . . . . 239

A.2.3 Heterogeneity . . . . . . . . . . . . . 239

A.2.4 Etiology . . . . . . . . . . . . . . . 240

A.3 Diagnostic Procedures and Investigations . . . . . . . . . . 240

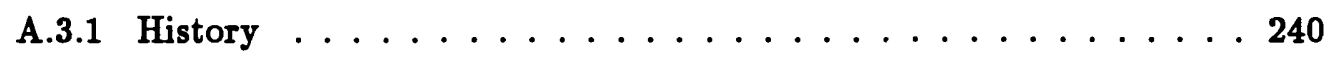

A.3.2 Basic Investigations . . . . . . . . . . . . . 240

A.3.3 The Diagnostic Procedure . . . . . . . . . . . . . 241

B CLOS Constructs for LDDB Representation of Eye Anomalies 244

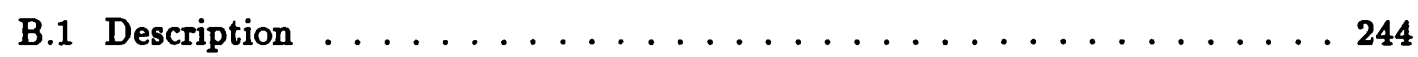

B.2 Eyes Object Declaration . . . . . . . . . . . . . . . . . 245

B.3 Eyes Object Generation Macro . . . . . . . . . . . . . . . 246

C Case Ordering Considerations for the C-UNIMEM Algorithm 247

C.1 Introduction . . . . . . . . . . . . . . . . . . 247

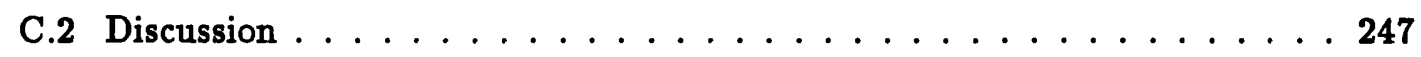

D Trace of Ranking and CBL Procedure with Case 564525 256 


\section{List of Figures}

1.1 Dysmorphology task analysis diagram. . . . . . . . . . . . 26

2.1 The LDDB Architecture. $\ldots \ldots \ldots \ldots \ldots \ldots \ldots$

2.2 The SDD Architecture. . . . . . . . . . . . . . . . 44

2.3 The diagnostic cycle of SDD. . . . . . . . . . . . . . . 47

2.4 Syndrome computer systems by category. . . . . . . . . . . . . . 59

2.5 The general CBR model. . . . . . . . . . . . . . . . . . . 65

2.6 A concept hierarchy in dysmorphology. . . . . . . . . . . . . . . 68

3.1 The four levels of syndrome nomenclature. . . . . . . . . . . . . 76

3.2 Software components of the general CBR architecture. . . . . . . . 78

3.3 Object case structure. . . . . . . . . . . . . . . 79

3.4 Different representations of a case co-exist. . . . . . . . . . . 81

3.5 Object structure is hidden from calling procedures via the utility programs. 84

3.6 Distributed storage of a case. . . . . . . . . . . . . 86

3.7 Object retrieval functions. . . . . . . . . . . . . . . . . 89

3.8 Retrieval of syndromes with (eyes iris coloboma), (neck general abnormal) and (neurology general abnormal) . . . . . . . . . . . . . 90

3.9 Hierarchical linkage of header objects. . . . . . . . . . . . . . . . . . 94

4.1 A sample discrimination hierarchy. . . . . . . . . . . . . . . . . . 99

4.2 The top level UNIMEM algorithm. . . . . . . . . . . . . . . . . . . . 102

4.3 The main control functions in UNIMEM. . . . . . . . . . . . . . . . 103

4.4 Idiogram of the number 5 chromosome. . . . . . . . . . . . . . . 108

4.5 Network A.1: Concept hierarchy generated by the Acrocephalosyndactyly data set. . . . . . . . . . . . . . . . . . . 111 
4.6 Network S.1: Concept hierarchy generated by the skeletal dysplasias data set. . . . . . . . . . . . . . . . . . . . . . . . . 112

4.7 Mean GAF achieved by UNIMEM. . . . . . . . . . . . . . . . 115

4.8 Network C5.1: Concept hierarchy generated by cases with a duplication in the number 5 chromosome $(77$ cases $) \ldots \ldots$. . . . . . . . . . 119

4.9 Network C2.1: Concept hierarchy generated by cases with a deletion in the number 2 chromosome (72 cases). . . . . . . . . . . . . 120

4.10 Diagnostic search with Network A.1 . . . . . . . . . . . . . . 124

5.1 Modified (weighted) UNIMEM algorithm. . . . . . . . . . . . . . 137

5.2 Network A.2: Revised concept hierarchy generated by the Acrocephalosyndactyly data set. . . . . . . . . . . . . . . . 142

5.3 Network S.2: Revised concept hierarchy generated by the skeletal dysplasias data set. . . . . . . . . . . . . . . . . . 143

5.4 Network C5.2: Revised concept hierarchy generated by cases with a duplication in the number 5 chromosome. . . . . . . . . . . . . . . . . 144

5.5 Network C2.2: Revised concept hierarchy generated by cases with a deletion in the number 2 chromosome. . . . . . . . . . . . . . . . 145

5.6 Comparative mean GAF achieved by UNIMEM and W-UNIMEM. . . . 146

5.7 A general CBL system . . . . . . . . . . . . . . . . 150

6.1 Top level C-UNIMEM algorithm and generalise function. . . . . . . . 165

6.2 Evaluation contrast parameters: Acrocephalosyndactyly group. . . . . . . 166

6.3 Generalisation contrast parameters: Acrocephalosyndactyly group. . . . . 167

6.4 Network A.3: Concept hierarchy generated by the Acrocephalosyndactyly data set. . . . . . . . . . . . . . . . . . . . 167

6.5 Evaluation contrast parameters: skeletal dysplasias group. . . . . . . . . 169

6.6 Generalisation contrast parameters: skeletal dysplasias group. . . . . . . . 170

6.7 Network S.3: Concept hierarchy generated by the skeletal dysplasias data

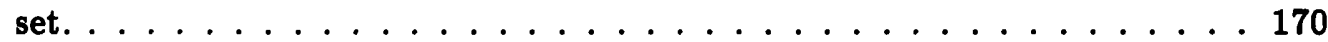

6.8 Comparative mean GAF achieved by UNIMEM, W-UNIMEM and CUNIMEM. . . . . . . . . . . . . . . . . . . . . . 171

6.9 Network C5.3: Concept hierarchy generated by cases with a duplication in the number 5 chromosome. . . . . . . . . . . . . . . . 173 
6.10 Network C2.3: Concept hierarchy generated by cases with a deletion in the number 2 chromosome. . . . . . . . . . . . . . . . . 174

6.11 Network W.1: Concept nodes formed by Clinician A and Clinician B with

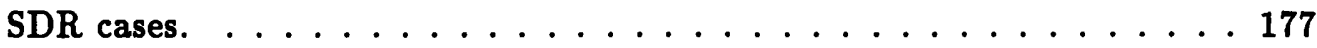

6.12 Network H.1: Concept nodes formed by Clinician C with SDR cases. . . . 179

6.13 Mean GAF for Networks S.1, S.2, S.3, W.1 and H.1 . . . . . . . . 181

7.1 A general CBR model for dysmorphology. . . . . . . . . . . . . . 198

7.2 Ranking contrast parameters. . . . . . . . . . . . . . . 201

7.3 Application of CBL to localised sections of the case-base. . . . . . . . 214

A.1 Phenotype analysis in dysmorphology. . . . . . . . . . . . 238

C.1 Network R.1 . . . . . . . . . . . . . . . . . . . . . . . 249

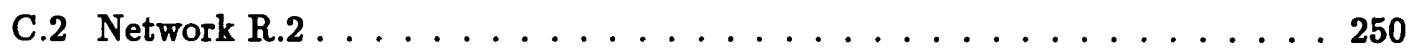

C.3 Network R.3 . . . . . . . . . . . . . . . . . . . . . . . 251

C.4 Mean $G A F$ for Networks A.3, R.1, R.2 and R.3 . . . . . . . . . . 255 


\section{List of Tables}

1.1 Main abnormalities of Down syndrome. . . . . . . . . . . . 20

2.1 Clinical regions represented by LDDB. . . . . . . . . . . . . . 39

2.2 LDDB representation of eye abnormalities. . . . . . . . . . . . 40

2.3 Syndromes returned by feature set (07.06.03 AND 15.01 AND 32.01). . . 41

2.4 Comparative procedures of dysmorphology, CBR and incremental concept formation. . . . . . . . . . . . . . . 71

3.1 Number of stored objects per clinical region for 1885 LDDB syndromes. . $\quad 88$

4.1 Five objects defined by attributes: colour, shape, size and orientation. . . 100

4.2 Acrocephalosyndactyly and Acrofacial Dysostoses data set. . . . . . . . 107

4.3 Symbolic feature descriptions for Case 7877 . . . . . . . . . . . . . 107

4.4 Skeletal dysplasias data set. . . . . . . . . . . . . . . . . . 107

4.5 Radiological items represented in SDR. . . . . . . . . . . . . . . 108

4.6 Node descriptions for Network A.1 . . . . . . . . . . . . . . . 113

4.7 Description of Node N21 in Network C5.1. . . . . . . . . . . . 121

4.8 Hypothetical case with craniosynostosis. . . . . . . . . . . . . 123

4.9 Revised node descriptions for Network A.1 . . . . . . . . . . . 125

5.1 Parameter settings for Acrocephalosyndactyly, $\operatorname{Dup}(5)$ and $\operatorname{Del}(2)$ data sets.139

5.2 Parameter settings for skeletal dysplasias data set. . . . . . . . . . . 139

5.3 Node N4, N4.1, N5 and N5.1 descriptions from Network S.2 . . . . . . . 148

6.1 Contrast between Case 5754 and Node N5 of Network A.3. . . . . . . . 168

6.2 Clinicians A and B group descriptions of SDR cases. . . . . . . . . . 178

6.3 Clinician $\mathrm{C}$ group descriptions of SDR cases. . . . . . . . . . . . 180 
7.1 Position of correct diagnosis in ranked differential for Acrocephalosyndactyly and Acrofacial Dysostosis cases. . . . . . . . . . . . 202

7.2 Position of correct diagnosis in ranked differential for Acrocephalosyndactyly and Acrofacial Dysostosis cases with $E_{t}=20.0 \ldots \ldots 203$

7.3 Position of correct diagnosis in ranked differential for Nager and TreacherCollins cases with $\theta=1.5 \ldots \ldots \ldots$. . . . . . . . . . . . . . . . .

7.4 Case 8967 (Treacher-Collins syndrome). . . . . . . . . . . . . 207

7.5 Ranked differential diagnosis for Case 8967. . . . . . . . . . . . . 207

7.6 Case 564525 dysmorphic features. . . . . . . . . . . . . . . . . . 211

7.7 Memory items which list craniosynostosis, abnormal fingers, and general eye abnormalities. . . . . . . . . . . . . . . . . . 212

C.1 Four case orderings for Acrocephalosyndactyly data set. . . . . . . . . 248

C.2 Node descriptions for Network R.1 . . . . . . . . . . . . . . . . 252

C.3 Node descriptions for Network R.2. . . . . . . . . . . . . . 253

C.4 Node descriptions for Network R.3. . . . . . . . . . . . . . . . . . . . 254 


\section{Chapter 1}

\section{Introduction to Dysmorphology}

\subsection{Thesis Overview}

A field of artificial intelligence (AI) that has grown in popularity over the last decade is case-based reasoning (CBR). Case-based reasoning is linked with the sub-field of AI known as machine learning because it is a model for knowledge-based systems that are 'dynamic'. That is to say, CBR systems update their knowledge-bases, and are thus viewed to learn. A crucial feature of CBR systems is that they comprise, amongst other functional units, a 'case memory' which stores actual cases, or exemplars, with which to compare and solve new problem situations.

Case-based reasoning has been applied to many fields, however, medicine is often cited as a classic CBR domain. This premise is due to the fact that many medical fields involve diagnosis by comparison with previous patients, or a set of prototypical patient symptoms (i.e., generalised cases). Thus, problems are addressed (but not always solved) by analysis against precedents, i.e., a case-based reasoning approach. The dysmorphology domain is one field of medicine that utilises such a process in diagnosis. Diagnosis of an affected infant is performed through comparison with previous patients or prototype entities known as syndromes. However, dysmorphology is a field of medicine in which knowledge of the domain is incomplete. Consequently, it is a field in which specialists perform a dual role involving both diagnosis and research. Research in dysmorphology pertains to the task of learning new dysmorphic syndromes (i.e., disorders that were previously unclassified, or undefined). A tenet of this thesis is that the tasks of diagnosis and research in dysmorphology can be mapped to theoretical ideas of case-based reasoning 
and learning, and this thesis makes such a mapping. The work described in this thesis involves the development of a case-based reasoning system that is designed to assist a specialist in dysmorphology, both in diagnosis and learning new syndromes. Diagnostic computer systems in dysmorphology are not new. However, the work described in this thesis is distinct in that it specifically addresses the research oriented learning aspect of the field.

The remainder of this chapter introduces the principal aspects (and problems) of diagnosis and research in the dysmorphology domain. This allows the specific goals of the thesis to be expressed with regard to the key medical performance tasks that have provided the motivation for this research. Chapter 2 reviews previous computer applications in dysmorphology. A comprehensive review of case-based reasoning was considered inappropriate due to the (increasing) wealth of CBR reference material that is available. An up to date review of computer systems in dysmorphology was considered, therefore, to provide a more worthwhile contribution to the thesis ${ }^{1}$. The basic principles of CBR are presented at the end of Chapter 2 with reference to the relevant aspects of the dysmorphology domain.

Chapter 3 is perhaps the most technical chapter in the thesis in that it refers to some important aspects of the system code (written in Common Lisp). This chapter describes the underlying architecture that provides the platform for the later work. However, its design does constitute an important aspect to the work. One of the problems inherent within dysmorphology is the lack of availability of uniform data. Chapter 3 describes an architecture that has been specifically designed to operate with non-uniform data originating from different sources.

Chapters 4 through 6 focus on case-based learning (CBL). More specifically they describe the development and enhancement of a case-based learning algorithm designed to operate with real data from the dysmorphology domain. The aim, in this instance, is to develop a system capable of assisting an expert user specifically with regard to the research aspect of dysmorphology, i.e., learning new syndromes. The very real-world nature of the data (reflecting the fact that an attempt is being made to map CBR to a real-world domain) presents a number of practical issues with regard to case-based learning, and these are discussed accordingly throughout these three chapters.

Whilst Chapters 4 through 6 focus on learning procedures in particular, it is impor-

\footnotetext{
${ }^{1}$ The most recent review of computers in dysmorphology, by Diliberti [16], is somewhat out of date.
} 
tant to remember that the primary task for a physician is diagnosis. Therefore, if an application aims to provide practical assistance to a specialist, the diagnostic task should not be overlooked. This work does have such a practical aim, and with this goal in mind Chapter 7 presents a general CBR model designed to provide assistance in both diagnosis and research. This model incorporates the work reported by Chapters 3 through 6. Finally, Chapter 8 concludes the thesis by summarising the relevant contributions to the designated areas of research, and discussing the potential for future work within this field.

\subsection{An Introduction to Dysmorphology}

Dysmorphology is that field of medicine which has as its concern the diagnosis of children born with multiple malformations. To this end dysmorphology involves the study of defects in morphogenesis, the various processes occurring during the development of the form and organs of the body. A pattern of malformations recognised as occurring together and thought to be pathogenetically related is called a syndrome. A number of generic terms used interchangeably to describe this entity, such as multiple congenital anomaly syndrome and dysmorphic syndrome. Malformations, which are also referred to as dysmorphic features, abnormalities or anomalies, are not necessarily visible physical defects. They may pertain to clinical, radiological, biochemical, histological or chromosomal defects. This diversity is reflected by the relevant diagnostic expertise, which is sparse and provided by specialists from varying disciplines such as clinical medicine, genetics and radiology.

It has been estimated that 8 in 1000 of children are born with multiple malformations [85]. Furthermore, about half of these infants will be linked to a chromosomal disorder recognised by performing a karyotype ${ }^{2}$. The diagnostic process effectively terminates if a recognised chromosomal abnormality is found, as this will be used to categorise the condition. If no chromosomal abnormality can be isolated, and the karyotype appears normal, the task of diagnosis becomes more difficult. The main issues concerning diagnosis will be discussed later in this chapter. At this stage it is suffice to say that diagnosis is performed by specialists who have at hand not only modern medical techniques, such

\footnotetext{
${ }^{2}$ A photomicrograph arranged to show the chromosomes in descending order of sire. Special staining of chromosomes delineates bands of various width on the regions, or loci, of the chromosomes which facilitates analysis of potential abnormalities.
} 
as chromosomal analysis, biochemical testing and diagnostic imaging, but also much experience in examining and diagnosing patients with dysmorphic syndromes.

There are currently over two and a half thousand recognised non-chromosomal syndromes (although there is a belief within the field that a number of these may eventually be linked with a chromosomal defect, but as yet cannot be so categorised due to the limitations of chromosomal analysis). Diagnosis of children with a normal karyotype is achieved by matching the patient with one of the recorded syndromes. In order to do this, the pattern of anomalies exhibited by the infant must be determined and then used to link the patient to a recognised disorder. For some cases, a firm diagnosis may be quickly established by an experienced specialist, particularly if the condition is characterised by one or more extremely significant anomalies. For other cases, an initial examination may result in a differential diagnosis, which comprises a list of dysmorphic syndromes that are characterised by features exhibited by the patient. Further investigation may allow the correct syndrome to be isolated ${ }^{3}$, or enable the differential diagnosis to be refined to the most probable candidates. This leads to an important aspect of the dysmorphology domain, certain syndromes are thought to be related. They are perceived to be related in the sense that they exhibit similarities in their pattern of anomalies. Related syndromes are described by dysmorphology specialists as forming families. It is not true to say that a differential diagnosis will only, or usually, occur between syndromes of the same family. A differentiation will be necessary when a patient exhibits sufficient similarity to more than one syndrome irrespective of whether those syndromes are thought to be related. However, it is important to appreciate that some disorders appear to form groups. A situation may arise in which it is difficult to diagnose a patient to an individual syndrome, but there is evidence to link the patient to the more general group. In such a case, the disease would remain undiagnosed until there was greater understanding of how individual syndromes may be distinguished within the syndrome family.

As indicated above, not all individuals with multiple malformations can be diagnosed precisely. In fact, the two and a half thousand or so known syndromes only account for about sixty per cent of cases. Of the rest, some patients may be recorded as isolated cases that bare little resemblance to any of the known syndromes, whereas others may display some degree of similarity to a known syndrome, or syndrome family, but not sufficient to

\footnotetext{
${ }^{3}$ It is possible for more than one syndrome to be exhibited by an infant, however, this occurrence is rare.
} 
establish a diagnosis. This phenomenon may be due to the lack of a specific abnormality known to have particular relevance to the disorder under consideration, or perhaps may be due to a finding which is not apparent in the syndrome description. Either way, there is sufficient dissimilarity to cause doubt about the match between the patient under examination and the syndrome under consideration. This aspect of dysmorphology results in a secondary role for dysmorphologists, that of researcher. Formal recognition of new syndromes is an active process within dysmorphology. However, due to the sparsity of expertise, and the paucity of individual patients, recognition and registration of new syndromes tends to take place gradually over many years. Reference materials including books $[30,75,85,51,22]$, journals $[92,93,94,95,96]$ and computer databases ${ }^{4}$ are available which document individual cases as well as providing comprehensive diagnostic compendia of known syndromes. These endeavour to assist specialists in investigating a potential diagnosis, and to some extent can be an important aid when the relationship between syndromes is under scrutiny.

As a final comment on this brief overview of dysmorphology, it is important that one does not overlook the question of purpose, i.e., what is the aim of diagnosing a patient as having a particular syndrome, and what are the benefits of recognising a new syndrome? Within dysmorphology these questions have implications further than the more straightforward answer of enabling a plan of management or course of treatments for the patient. For certain known conditions a firm diagnosis may provide a prognosis. If the disorder is less common, a prognosis is not always possible, and certainly becomes more difficult. Recognition, categorisation and documentation of new disorders will help some way towards increasing knowledge and perhaps ultimately allow a prognosis for a greater number of dysmorphic syndromes. The dysmorphologist has one further important role, that of genetic counsellor. With the more common syndromes, genetic counseling may involve informing the parents about the expected development of the disease, and perhaps informing them of the risk of recurrence if they were to have another child. In all cases, whether a course of treatment is possible, limited, or cannot be formulated, the parents of the child will at least seek a name for the condition. Diagnosis may provide the name of a known syndrome. If the case is undiagnosed, or is associated with a rare syndrome, the physician may have greater difficulty in indicating a specific cause, or etiology, for the disorder. The ability to understand in some way, even merely by a label, can be of

\footnotetext{
${ }^{4}$ A review of compater applications is given in Chapter 2.
} 
great importance to the parents. It can provide a means by which to communicate about the disease, or perhaps to examine the literature themselves.

The following section is intended, by way of an example, to draw a more detailed picture of the kind of conditions described by terms such as congenital anomaly syndrome and dysmorphic syndrome. One of the most common and widely recognizable syndromes is Trisomy 21 syndrome, or Down syndrome. A description presents the main diagnostic signs of this condition as presented in one of the standard syndrome compendia, Smith's Recognizable Patterns of Buman Malformation [30]. Along with a description of the disease, some of the important issues concerning diagnosis are addressed which are relevant to Down syndrome, but are applicable to dysmorphic syndromes in general.

\subsection{Down Syndrome (Trisomy 21 Syndrome)}

Down syndrome is one of the oldest recorded patterns of multiple anomalies, and one of the most common (an incidence of 1 in 660 newborns). Down syndrome etiology is associated with a chromosomal defect, specifically trisomy ${ }^{5}$ of chromosome 21 . As this defect would be apparent when examining a karyotype, which would in turn provide sufficient proof for a diagnosis of Down syndrome, a diagnostic procedure involving examination of dysmorphic features may not be necessary. However, there is no significant difference in how chromosomal and non-chromosomal syndromes are documented, apart from the fact that with chromosomal disorders the etiology is apparent and with nonchromosomal syndromes it is not. Discussion of diagnostic procedures with respect to dysmorphic features is valid for all syndromes.

On examination of a patient with Down syndrome, a dysmorphologist may record a variety of anomalies such as congenital mental retardation (which can range from moderate to severe), a sloping forehead, low set ears, small ear canals, oriental appearance of the eyes, gray or very light spots at periphery of iris (known as Brushfield Spots), short broad hands with a single palmar crease (or simian crease ${ }^{6}$ ), a flat nose or absent bridge and generally dwarfed physique. These signs would all support the diagnosis. In the literature, syndrome descriptions are documented more systematically and may include clinical and radiological photographs of recorded patients with family histories. Table 1.1

\footnotetext{
${ }^{5}$ Having three of a particular chromosome instead of the usual two.

'A simian crease is a fusion of two of the transverse creases on the palm of the hand into one.
} 


\begin{tabular}{|c|c|}
\hline General & $\begin{array}{l}\text { Hypotonia (relaxcation of muscles and pressure of arteries), relatively } \\
\text { small stature with awkward gait, a tendency to keep mouth open and } \\
\text { protrude the tongue, hyperflexibility of the joints, and diastasis recti } \\
\text { (a lateral separation of the two halves of the rectus abdominal muscles). }\end{array}$ \\
\hline $\begin{array}{l}\text { Central Nervous } \\
\text { System }\end{array}$ & Mental deficiency, or retardation. \\
\hline Craniofacial & $\begin{array}{l}\text { Brachycephaly (a short head) with a relatively flat back part of } \\
\text { the skull, mild microcephaly (abnormal smallness of the head) with } \\
\text { upslanting palpebral fissures (the opening between the eyelids), a } \\
\text { thin cranium with late closure of fontanels (unossified space or } \\
\text { soft spot lying between the cranial bones of the skull), hypoplasia } \\
\text { (underdevelopment) to aplasia (the failure to develop normally) of } \\
\text { the frontal sinuses, a short hard palate, a small nose with a low } \\
\text { nasal bridge, and inner epicanthal folds (vertical folds of skin } \\
\text { extending from the root of the nose to the inner end of the eyebrow). }\end{array}$ \\
\hline Eyes & $\begin{array}{l}\text { Brushfield's spots with peripheral hypoplasis of the iris, opacity of } \\
\text { the fine lens, and refractive errors in the lens. }\end{array}$ \\
\hline Ears & $\begin{array}{l}\text { Generally small, sometimes prominent, with small or perhaps absent ear } \\
\text { lobes, and an overfolding of the upper helix part of the external ear. }\end{array}$ \\
\hline Teeth & $\begin{array}{l}\text { Hypoplasia, irregular placement, and fewer caries (areas of decay) } \\
\text { than usual. }\end{array}$ \\
\hline Neck & Appears short. \\
\hline Hands & $\begin{array}{l}\text { Relatively short metacarpals and phalanges (bones in the fingers), } \\
\text { clinodactyly (lateral deflection of the fingers), and a simian crease. }\end{array}$ \\
\hline Feet & $\begin{array}{l}\text { Wide gap between the first and second toes, a plantar crease between the } \\
\text { first and second toes (on the sole of the foot), and dermal ridge patterning } \\
\text { in the hallucal (big toe) area of the sole. }\end{array}$ \\
\hline Pelvis & $\begin{array}{l}\text { Hypoplasia, flared iliac wings (widest part of the pelvic bones), and a } \\
\text { shallow acetabular angle. }\end{array}$ \\
\hline Cardiac & $\begin{array}{l}\text { Atrioventricularis communis (the failure of the division of the } \\
\text { atrioventricular canal in the embryo), ventricular septal defect (defect } \\
\text { in the septum, or wall, between the left and right ventricles of the heart), } \\
\text { patent ductus arteriosus (a wide opening between the main pulmonary } \\
\text { artery and the aorta), auricular septal defect (a defect in the wall of the } \\
\text { atrium, or upper chamber of each half of the heart), and aberrant } \\
\text { subclavian artery (abnormal deviation of the artery beneath the clavicles). }\end{array}$ \\
\hline Skin & $\begin{array}{l}\text { Loose folds at the back of the neck, cutis marmorata (purplish } \\
\text { discoloration of the skin on exposure to cold), dry skin, and hyperkeratotic } \\
\text { skin (overgrowth of the outer layer of skin) with time. }\end{array}$ \\
\hline Hair & Fine, soft and often sparse, straight pubic hair at adolescence. \\
\hline Genitalia & $\begin{array}{l}\text { In males a relatively small penis. Hypogonadism (defective internal } \\
\text { secretion of the gonads). }\end{array}$ \\
\hline
\end{tabular}

Table 1.1: Main abnormalities of Down syndrome. 
lists the main abnormalities associated with Down syndrome.

\subsubsection{Diagnosticity and Occasional Features}

Table 1.1 provides a comprehensive set of anomalies that anfant with Down syndrome might exhibit. A pattern of features from this set may together distinguish Down syndrome from other known disorders. A patient may not necessarily exhibit all the abnormalities listed, although a sufficiently conclusive subset of typical features must generally be evident in order to establish a diagnosis. The significance of each individual feature with respect to the diagnosis will vary. Some features are more important than others in order to establish, or distinguish, a syndrome, and therefore carry greater diagnostic weight. Indeed, an abnormality may be so diagnostic to a particular syndrome that it may be considered essential for that particular diagnosis. The existence of very diagnostic features may enable a specialist to focus very quickly on a particular syndrome. However, it is important to note that generally a set of features will together support the diagnosis (hence the term 'pattern' is used). An isolated anomaly might not be particularly diagnostic, but when seen along with certain other abnormalities its significance may increase. Thus, the diagnostic weight, or diagnosticity, of an individual feature can vary according to its known relationship with other anomalies.

There are no firm rules that govern diagnosticity, and it is a concept that suffers naturally from differences in interpretation. Winter and Baraitser suggest a mechanism for diagnosticity in terms of what they call good and poor 'handles' [84, 85]. Handles refer to specific dysmorphic features. Some basic rules proposed by Winter and Baraitser in order to determine what may or may not constitute a good handle are listed in Appendix A. This approach was introduced to complement the usage of their computer system, the London Dysmorphology Database [86]. The idea of diagnostic handles can be construed as a search strategy in which a handle provides a good index rather than a specific evaluation of diagnostic significance. For instance, a good handle should be a feature that is fairly infrequent across different syndromes so that a set of potential diagnoses that is small enough to be manageable may be found. Smith's compendium [30] also uses a statistical appraisal of dysmorphic features, and quotes the incidence of specific anomalies with respect to a specific disorder. Emphasis tends to be put on features with a high incidence. Thus, for both approaches, a feature that is frequent within a specific condition, but is rare elsewhere, will naturally carry a high diagnostic significance for 
that disorder. However, a correlation between frequency and diagnosticity cannot always be made, and should not be assumed. For example, mental retardation can occur in over six hundred different syndromes and so provide a poor handle by which to search for relevant syndromes. Conversely, it is such an important feature that it may be essential to the diagnosis of certain conditions.

This tentative link between diagnostic significance and incidence of occurrence is further demonstrated by what are termed occasional features. A pattern of malformations may occasionally cause other anomalies, sometimes thought of as a kind of developmental noise. The list of main abnormalities in Table 1.1 consists of defects that occur in at least 25 per cent and usually more than 50 per cent of patients. Smith denotes occasional anomalies as having a frequency of 1 to 25 per cent (most commonly 5 to 10 per cent), although anomalies with greater incidence may also be classed as occasional (a set of occasionals is often listed along with the main diagnostic pattern). The existence of an occasional feature for a patient will be of no surprise to an experienced physician who has seen many infants with Down syndrome. Occasional features are not random for a particular syndrome, and they do carry some diagnostic value, or weight, but this tends to be relatively low and can only be positive. That is to say, occasional features can only add support to a possible diagnosis. They do not carry enough weight in order for their absence to count against a diagnosis. Below is a list of occasional features that are specific to Down syndrome with an indication of their incidence.

- Seizures (less than 5 per cent).

- Strabismus, or squinting (33 per cent).

- Cataract (1.3 per cent).

- Nystagmus, an involuntary movement of the eyeball (15 per cent).

- Incomplete fusion of the vertebral arches of the lower spine (37 per cent).

- Cryptorchidism, a condition in which there is a failure of the testicles to descend into the scrotum ( 27 per cent from birth to nine years and 14 per cent after 15 years).

- Leukemia (approximately 1 per cent). 
As stated earlier, to formulate a diagnosis the dysmorphologist must use the pattern of patient findings to reference and match the patient with known syndromes (this may involve techniques such as diagnostic imaging along with clinical examination). An expert may be particularly familiar with certain syndromes or syndrome families, which may enable a differential diagnosis to be compiled almost immediately. Otherwise, the physician may have to search the literature for a match. Either way, to establish a match, the subject (the patient) and the referent (the syndrome) must match across a conclusive pattern of anomalies. A diagnostic set is a conclusive pattern of anomalies exhibited by the infant that can provide the necessary proof, or confidence, for a diagnosis. The anomalies that comprise the diagnostic set tend to be principal features ${ }^{7}$ of relatively high diagnosticity, although occasional features may be included. Smith's compendium notes the following set of principal features for Down syndrome based on high incidence in recorded cases. The list is intended as a guide for neonatal examination of the infant. Hypotonia (evident in $80 \%$ of cases), a poor Moro reflex ${ }^{8}$ ( $85 \%$ ), hyperflexibility of joints $(80 \%)$, excess skin on the back of the neck (80\%), a flat facial profile $(90 \%)$, slanted palpebral fissures ( $80 \%$ ), anomalous external ears (60\%), dysplasia ${ }^{9}$ of the pelvis $(70 \%)$, dysplasia of the midphalanx of the fifth finger $(60 \%)$, and a simian crease $(45 \%)$. For a conclusive diagnosis of Down syndrome a physician would expect to establish a diagnostic set comprising of anomalies from these principal features.

\subsubsection{Additional Diagnostic Information}

There are other contextual factors which may also provide diagnostic pointers. For instance, the sex of an infant can be very discriminating (some syndromes are known to have sex linkage with respect to inheritance, for example, Coffin-Lowry syndrome [51]). Age can also be important. The status of a child at presentation (or examination) refers to whether the infant is alive or not. The circumstances of a fatality (e.g., neonatal death or abortion) may have relevance to a diagnosis. If a child is alive, or has lived for a significant period beyond birth, then age can also be an important diagnostic pointer (as some syndromes are distinguished as surviving groups).

\footnotetext{
${ }^{7}$ Winter and Baraitser think in terms of hard features as abnormalities with high diagnostic significance, whilst soft features are equivalent to secondary and occasional abnormalities.

'A defensive reflex consisting of the infant's drawing of its arms across its chest in an embracing manner in response to stimuli produced by striking the surface on which the infant rests.

${ }^{\circ}$ A dysplasia refers to the abnormal development of the respective tissue.
} 
A further factor concerns the use of normal features during diagnosis. When considering a diagnosis, a physician will not generally refer to normal patient findings unless there is a direct conflict with syndrome expectations (for instance, if an abnormality is common in a syndrome, but the organ is normal for the patient). Thus, the existence of a normal finding in the patient can create a kind of negative diagnostic value, especially if a known anomaly is regarded as essential, thus ruling out the particular syndrome under consideration (this would only be true for principal features, occasional features may only carry positive diagnosticity). Whilst normality can, therefore, be (negatively) diagnostic, a problem may arise if a feature is minimally normal or abnormal. Exclusion of the correct diagnosis may occur because of an incorrect interpretation of a minor abnormality. It should be noted that there is some debate amongst dysmorphology specialists regarding this matter [61].

\subsubsection{Etiology, Prognosis and Genetic Counseling}

Establishing an etiology for a condition will, to a great extent, depend on the respective diagnosis. It follows also that a diagnosis will increase the chance of a prognosis and enable the physician to provide some degree of genetic counseling to the parents of the infant. A diagnosis of a condition such as Down syndrome will immediately suggest an etiology and enable a substantial level of counseling since it is a relatively common disorder with much documentation. For less common conditions, the cause may be more difficult to establish, and both the prognosis and counseling given to parents will have less certainty. At the extreme end of the scale, for cases that have no diagnosis or belong to a very rare group with few or no references available, it may not be possible to establish a prognosis, and so the information that can be given to parents will be restricted, for instance, the physician may not be able to predict the risk of recurrence.

If a child has Down syndrome, the natural history, or the expected progression of the child physically and mentally with age, is well established. For example, the physician can predict growth rate (which is generally slow for the first eight years, but becoming more normal beyond with final height achieved at around fifteen years of age), sexual development (females can be fertile, but males are generally infertile) and the probable I.Q. range (25-50). The physician may also be able to indicate the chances of early mortality (for example, 44 per cent of individuals with cardiac anomalies die in infancy, although between infancy and the first 40 years of age the mortality rate is not much 
greater than normal). The parents may be informed of the risk of recurrence which is generally around 1 per cent, although the age of the mother may be important. For example, the incidence for a maternal age between 15 and 29 years is 1:1500, whereas the incidence for a maternal age from 35 to 39 is 1:270.

\subsection{Performance Tasks in Dysmorphology}

This section provides an overview of the processes that are intrinsic to the two key tasks performed by dysmorphology specialists: diagnosis of an affected infant to a recognised syndrome, and investigation of new syndromes and existing syndrome categories. Figure 1.1 gives a diagrammatic representation of the main processes at work when performing these tasks.

\subsubsection{Diagnosis}

Diagnosis is in essence a classification task. The goal of diagnosis is to accurately classify a patient with reference to known syndrome categories. Classification implies a process of matching, or similarity assessment. Much of this skill emanates from general medical knowledge in combination with experience specific to the domain. To an experienced specialist some syndromes will be instantly recognisable and will not need a detailed analysis of the dysmorphic features (although an investigation will be required for confirmation). This ability will reflect the dysmorphologist's specialist medical background. For example, a radiology specialist will be familiar with syndromes that principally affect the skeleton, whereas a geneticist will tend to know about syndromes associated with a chromosomal disorder.

As mentioned above, during the initial examination of a patient the dysmorphologist may find an abnormality that is particularly diagnostic to a known syndrome or syndrome family. If instant recognition fails, the examiner must determine and record the specific individual dysmorphic features. Whilst recording the pattern of anomalies exhibited by the child, the physician may need to quantify certain features. That is to say, the physician must decide what features are truly abnormal and give an accurate associated description. At this point, an important problem within dysmorphology is highlighted, there is no universal representation of abnormal features [8]. Different experts interpret features differently. Both Smith [30], and Winter and Baraitser [85] stress the importance 
of establishing early on what are definite abnormalities with accurate descriptions. For example, hypertelorism ${ }^{10}$ can easily be confused with telecanthus (in which case the eyes appear widely spaced due to the laterally displaced inner canthal margins ${ }^{11}$ ), ears may be described as low set, but could actually only appear low set because they are posteriorly rotated [85]. The interpretation of anomalies has a far reaching effect in dysmorphology through documentation of individual cases. A physician with a rare case at hand may

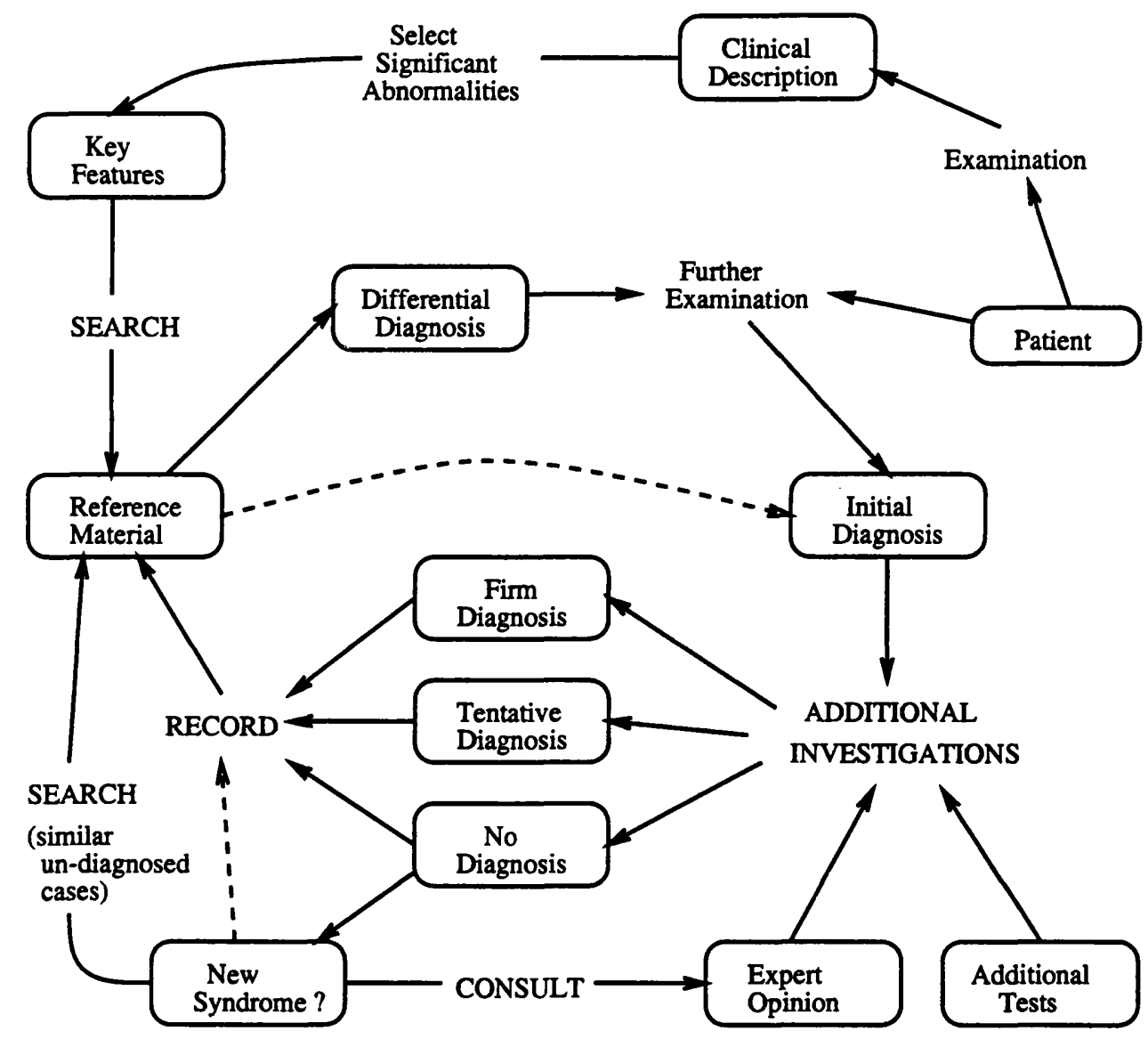

Figure 1.1: Dysmorphology task analysis diagram.

have to search the literature to find another similar case. Accurate documentation of anomalies is crucial to establish the similarity between two such cases (documentation will generally include photographs, which can ease this problem somewhat). Although some of the available texts and computer systems do attempt to standardise a nomenclature for abnormal features, the onus remains with each dysmorphologist to interpret abnormalities

\footnotetext{
${ }^{10}$ Abnormally large width between the eyes.

${ }^{11}$ The canthus refers to the angle at either end of the slit between the eyelids.
} 
correctly when recording a case.

The determination of patient anomalies may also involve detecting feature dependencies. It has been emphasised already that both patient and syndrome descriptions are defined in terms of patterns of anomalies. The importance of an individual feature may depend on its relationship with other anomalies in the overall feature set. Feature dependencies may be due to direct clinical or radiological relationships which would be known to medical experts. For instance, an infant may be described as having both short ribs and elongated clavicles. A radiology specialist would know that this correlation is usual. Of these features perhaps only one will be recorded which would probably the primary feature, short ribs. Then in any future references to this case, the secondary feature would need to be inferred by the examining physician. So as well as a non-uniformity in feature descriptions, there exists also a non-uniformity in case descriptions. That is to say, the detail in documented patient descriptions varies greatly. Some patients are recorded merely in terms of one or two principal abnormalities, whilst some case descriptions contain a more comprehensive list of dysmorphic features that were noted during examination. This type of non-uniformity can further complicate the task of matching rare cases.

Dependencies between anomalies may also pertain to observations of co-occurring features that have been recorded for certain conditions, or a number individual cases. These correlations may or may not have relevance to the diagnosis under consideration, and the examining physician may be required to examine the family history of the patient in order to establish any significance. Certain features may be evident as harmless traits in some families, for example, big thumbs and toes, which is a combination seen in Rubenstein-Taybi syndrome [85]. If a patient has big thumbs and toes it must be established whether or not these features were evident in normal, healthy ancestors before they are included in the overall pattern of anomalies (and therefore given diagnostic relevance). If the family history dictates that these features can exist normally, then the physician must carefully establish the other known features for Rubenstein-Taybi syndrome before proposing that diagnosis.

A clinical (or radiological) examination will hopefully determine a pattern of anomalies that is in some way ranked by diagnostic importance. The principal features will then be used to link the infant with known disorders, or perhaps more general syndrome families. Such a link may be created by searching through the relevant literature, or 
may result from specific knowledge, or experience, of the examining physician. If one particular syndrome stands out as the primary candidate, perhaps due to the existence of one or two particularly significant abnormalities, then further investigation may be focussed on establishing that diagnosis. For instance, further examination may be biased in the sense that there is a diagnosis 'in mind', and the physician may look for specific anomalies due to the diagnosis under consideration. This can lead to a quick diagnosis if the physician's suspicions are confirmed (by the existence of the relevant diagnostic set). However, if the respective anomalies cannot be found, the physician may be forced to reassess the proposed diagnosis. This can result in a reappraisal of the significance of anomalies recorded on initial examination.

If the recorded pattern of abnormalities matches a number of syndromes, then it may be necessary for the physician to refine this list. Further examination may take place, perhaps with greater significance placed on secondary and occasional features. This iterative process of examination and re-examination is ultimately geared towards proposing the most accurate form of diagnosis given the extent of the information at hand. For some affected infants, no diagnosis may be possible. In some cases, perhaps only a differential diagnosis is proposed. If the physician has a degree of certainty, then a firm, or tentative, diagnosis may be put forward.

At this point, given some form of initial diagnosis, the examining physician may seek further evidence through other means. Additional investigations can take on a number of guises. For example, initial clinical examination may have suggested a syndrome with a known skeletal anomaly, and so the physician may want to examine relevant $x$-rays, or consult with a radiologist. The cooperation between dysmorphologists specialising in different medical disciplines is an important necessity in diagnosis. Certain features may require the opinion of a specialist in a relevant field of medicine. For example, the

\section{Addendum}

It should be noted that whilst two primary tasks have been identified, i.e., diagnosis and research, the task of therapy following diagnosis is of critical importance, but extremely difficult in this field. 


\subsubsection{Research}

The role of research in dysmorphology is associated with the ever changing nature of the field and an associated desire to learn and understand more about it. Syndromes that once were thought to be very different, and were consequently classified separately, sometimes emerge as different manifestations of the same condition, or as the same condition at different points in its natural history. Alternatively, conditions that appear the same may, at a later date, be found to have very different underlying dynamics and prognoses. By monitoring both diagnosed and undiagnosed cases over time, the dysmorphologist may be able to spot previously unregistered correlations and establish previously unknown etiologies. Documenting the development of affected infants may enable more accurate prognosis and counseling in future.

Along with the compendia cited earlier, which are designed with an emphasis on diagnosis, the dysmorphologist also has at hand numerous research publications that can assist investigation $[92,93,94,95,96]$. These journals provide a medium by which to communicate with other specialists. There are also a number of annual conferences and workshops for medical specialists to attend. Communication is crucial in order to allow practitioners to announce new findings, discuss potential links between rare cases, and ultimately propose new syndromes (or establish revisions of known categories).

One of the results of documentation, analysis and communication with respect to cases of multiple malformations has been a nomenclature for syndromes and syndrome families. That is to say, there has been some thought towards higher order syndrome taxonomies. However, in keeping with the uncertain and ever changing nature of the field, nomenclatures are not interpreted rigorously. Compendia such as Smith's are structured in a way that reflects syndrome nomenclature. In Smith's book, syndromes are grouped across twenty or more general categories such as chromosomal abnormality syndromes, syndrome characterised by a very small stature, syndromes with facial defects as a major feature, craniosynostosis ${ }^{12}$ syndromes and osteochondrodysplasias ${ }^{13}$. Specialists within radiology have denoted a more specific classification of osteochondrodysplasias [69]. This working group has defined a taxonomy consisting of three general groups: defects of the tubular (and flat) bones and/or axial skeleton, disorganised development of cartilagenous

\footnotetext{
${ }^{12}$ Premature closure of the skull sutures

${ }^{13}$ Skeletal dysplasias pertaining to bone and cartilage. Dysplasias can also affect single bones.
} 
and fibrous components of the skeleton, and idiopathic osteolyses ${ }^{14}$. Within the first group affecting tubular bones, twenty four sub-categories have been defined. Similarly osteolyses are divided along four sub-groups. An important point about these syndrome groupings (which has relevance to issues discussed in Chapter 2) is that they effectively provide a category hierarchy. However, although a hierarchical classification of syndromes is conceivable, there is some reluctance to adhere to hierarchical schemas. The existence of syndrome clusters is generally accepted, but individual syndromes are not necessarily mutually exclusive. That it is to say, some syndromes may belong to more than one higher order category. It would seem also that hierarchies pertain only to relatively well understood syndrome families.

The specific procedures of learning involve the revision of a syndrome description or a higher order syndrome category. Such revision can occur in a number of ways. One process is the straightforward addition of a new disorder to the syndrome nomenclature, either with or without reference to existing categories. A new syndrome definition may be based on a link between two or more undiagnosed infants that stand isolated with reference to the existing syndrome nomenclature. It is important to note that some known syndromes are based on the descriptions of only a few cases (an example of this instance is Pfeiffer syndrome). These are precisely the syndromes where diagnosis is the most difficult. A new syndrome may also be defined with reference to a known disorder or family of disorders. If an individual has similarity to a known syndrome family, but not specifically to one of the family members, then it may be tentatively linked with the syndrome group until there is a greater understanding of that group. As further similar cases are registered and placed in the same vicinity of the nomenclature, a new, distinct syndrome may be formed within the syndrome family.

Category information may also be revised as more infants become diagnosed. The level of detail of syndrome descriptions can vary according to the number of diagnosed children. This is evident with the more common conditions for which statistical information is sufficient to provide accuracy (and confidence) in the syndrome description used for diagnosis. Descriptions of syndrome families also change over time. The definition of a syndrome is formed as a generalisation of the features by which diagnosed cases are adjudged to be similar. As more cases become registered, it may become apparent to the investigator that the group may be divided along certain traits, whilst still demonstrating

\footnotetext{
${ }^{14} \mathrm{~A}$ softening and destruction of bone tissue without recognised cause.
} 
some higher order relationship. Thus, knowledge may be derived in two directions: by generalising across a set of similar cases in order to establish a diagnostic set ${ }^{15}$, and by increasing the specificity amongst a set of related cases.

\subsection{Conclusions and Goals of the Research}

Dysmorphology is a complex and diverse medical domain. The state of the domain is incomplete, or weak, in the sense that there are many unknown cases and the level of understanding of dysmorphology is not total. It is very much a dynamic field of medicine with knowledge about syndromes increasing gradually over time. Thus, dysmorphology is not merely a domain for medical diagnosis, but also one of medical research. Practitioners, whether performing diagnoses or acting as researcher, are medical experts who rely on both general and specialised medical knowledge, and experience.

There has been a natural progression from texts to computer systems in order to provide support and assistance to the dysmorphologist. The following chapter reviews computer applications dedicated to dysmorphology. These vary in design and consequently in their scope. Some are merely syndrome databases. Some, on the other hand, employ artificial intelligence techniques in order to aid diagnosis, and may therefore be regarded as expert systems. One aspect common to all these computer systems is that they address the primary task of diagnosis, either through an automated diagnostic procedure or simply through a look-up facility. Some address the potential of using computer systems to learn new syndromes. However, none have specifically tackled this problem. Such investigation is left as an implicit task for the user.

The general theme of this research is to increase the scope of assistance available to a specialist in dysmorphology from a computer system. However, it is the learning aspect of the domain, i.e., the recognition of new syndromes and the revision of syndrome categories, that provides the specific focus of this work. In particular, the thesis involves the development of a computer system that utilises 'case-based reasoning'. Case-based reasoning (CBR) is a field of artificial intelligence (AI) linked to machine learning. An important feature of CBR systems is that they have the ability to learn automatically. That is to say, they can update their knowledge, which is stored in the form of specific and generalised cases, dynamically. A specific aim of the work is to automate the

\footnotetext{
${ }^{15}$ Generalisation may infer links between previously distinct syndromes.
} 
learning process of dysmorphology by designing and implementing a CBR system. Thus, unlike previous applications, this work specifically addresses the secondary learning task performed by dysmorphology specialists. The ability of a CBR system to learn automatically depends to a great extent on the structure of the case database, or case-base, and the methods for assessing the similarity between individual cases. To this end, CBR has much overlap with another field of machine learning called 'incremental concept formation'. Following the review of dedicated computer systems in Chapter 2, the main features of these two AI disciplines are discussed with reference to the dysmorphology domain.

It has been stated that the main focus of research is an automated, case-based reasoning approach to the learning task of dysmorphology. With this in mind, a background goal of the work is to design a practical solution which enables the primary task of diagnosis to be performed as well as specifically automating the learning process involved with the proposal of new syndromes. An important tenet of this research is that both diagnosis and learning can be represented using case-based reasoning. Furthermore, the same underlying case-based system architecture can be utilised by both 'calling procedures': object retrieval (i.e., retrieving similar syndrome or case entities from memory), and a learning CBR program (called case-based learning, or CBL). Chapter 3 describes this architecture, which lays the foundation for the subsequent chapters specific to case-based learning, and the general CBR model which is discussed in Chapter 7.

Whilst aspects of dysmorphology map well with facets of case-based reasoning, the complexities of the domain provide a challenge to some of CBR's underlying assumptions, particularly with respect to learning processes that rely on a single category hierarchy. Chapters 4 through 6 describe the application of a case-based learning program to dysmorphology. Chapter 4 reports on the implementation of an algorithm taken from the machine learning literature. The results from this experiment drive the work reported in Chapters 5 and 6, which describe extensions to the learning algorithm to improve its performance. Chapter 5 describes how the performance of the algorithm is improved when additional domain knowledge is available. Chapter 6 demonstrates how a similarity model taken from the psychology literature can be also integrated within the system in order to enhance the similarity assessment functions that are essential to the learning process. These three chapters are specific to a stand-alone case-based learning program which, whilst reported in the context of a general CBR solution, provides a specific focus 
on machine learning. They assess, and enhance, a recognised learning algorithm with respect to a real-world, and incomplete medical domain. Added to this, Chapter 6 considers (and answers) some of the open issues of similarity assessment with regard to CBR in the light of the work that has been done.

Finally, Chapter 7 describes a case-based reasoning system that facilitates diagnostic assistance as well as the research objective of learning new syndromes. This design provides a general CBR solution, derived from the work involved with learning algorithms (Chapters 4, 5 and 6), and the case-based architecture described in Chapter 3.

\subsection{Summary}

The following points summarise the main characteristics of the dysmorphology domain and goals of the thesis.

- The domain is incomplete. The acquisition of knowledge concerning dysmorphic syndromes is ongoing and is performed by experienced specialists of varying medical backgrounds.

- Two performance tasks exist: diagnosis and research. Both involve a process of assessing the similarity between two entities. Diagnosis involves matching a patient to known syndrome malformation patterns. Research involves comparing undiagnosed infants with a view to learning new syndromes.

- Whilst syndrome category structure is very loosely defined, generally a matter of interpretation, and not rigidly adhered to by domain experts, syndromes do appear to form groups, or families. The domain is not yet at a stage at which a single hierarchical categorisation can be defined.

- Previous computer systems dedicated to dysmorphology have addressed only the diagnostic task. The research task has not specifically been automated to any degree. This work addresses learning in dysmorphology. More specifically it involves the design and implementation of a computer system that automates this mode of operation.

- Previous computer systems comprise standard database and knowledge engineering approaches to design. This work utilises a case-based reasoning design, which 
maps well with facets of the dedicated domain and facilitates automated learning of syndromes and revision of syndrome categories.

- The main thrust of the work is to design and implement a model of case-based reasoning, specifically focussed at addressing the learning task. A standard CBL model is taken as an initial starting point, implemented, tested with data from the dysmorphology domain, and extended in order to improve performance.

- The complexities of the dysmorphology domain expose problems with regard to the case-based learning model and the theoretical assumptions on which it is founded. These issues are addressed, and solved by a more general case-based reasoning system that incorporates the CBL program, but also offers functions aimed at providing asisstance with the primary task of diagnosis. This approach is driven by a desire to provide a case-based system that offers a practical solution with regard to both diagnosis and research in dysmorphology.

\section{Addendum}

Some of the computer systems reviewed in Chapter 2 have employed statistical techniques. Certain problems that underline these techniques are discussed. This thesis describes work that has adopted CBR as its underlying model rather than statistical methods, which is enlarged upon in Section 2.6. 


\section{Chapter 2}

\section{Dysmorphology, Computer Systems and AI}

\subsection{Introduction}

The utilisation of reference materials (books, journals and computer systems) is an important, if not crucial, step in both diagnosis and research. The general structure of syndrome compendia has already been described in the previous chapter. This chapter reviews computer applications within dysmorphology. Some of these applications are essentially computerised equivalents of compendia, i.e., database systems. A typical database system would offer increased efficiency in a search for possible syndromes or similarly affected infants (if stored in the database) by using a relevant keyword indexing mechanism. A number of researchers, however, have endeavoured to extend the basic performance goal of a computer system beyond that of a straightforward look-up facility. In such cases energy has been directed towards modelling some of the processes at work during diagnosis.

It has already been concluded that dysmorphology is a difficult domain for medical diagnosis because knowledge of the domain is incomplete. Consequently, difficulties arise when attempting to model the procedures undertaken by medical specialists in order to capture them in a computerised format. There are no firm rules governing diagnosis, and there is much reliance on experience. Thus, the ensuing knowledge elicitation, however performed, may in itself prove a very difficult task. The strength of the resulting model will depend on the scope of the performance goal. If one is aiming to build a general 
diagnostic system, applicable across all known syndromes, then one can probably expect to establish a weak diagnostic model which at best provides a manageable differential diagnosis (which is small enough to refine through further examination). This is the realistic scope of a database application. On the other hand, as has already been indicated, syndromes do appear to form groups which may be described or specified across certain chromosomal, clinical or radiological criteria. If a system is intended to follow a diagnosis procedure for a specific, distinctive group, then it may be feasible to acquire sufficient knowledge from a specialist who is particularly familiar with that group in order to create a strong diagnostic model. This would allow the development of a more intelligent system that produces greater accuracy in the differential diagnosis.

The following sections describe two systems in detail: the aforenamed London Dysmorphology Database which, in terms of operation, typifies a database application, and the Skeletal Dysplasia Diagnostician [15, 36], a model-based expert system which demonstrates a knowledge engineering approach to development of a system dedicated to the diagnosis of a specific group of syndromes. These two systems correspond to the two above mentioned extremes in terms of system design and operation. Other computer applications are subsequently discussed with reference to these two systems. The topic of discussion will then switch to theoretical ideas from within artificial intelligence (AI) that are relevant to this research, and in particular to the system design issues that have been highlighted.

\subsection{The London Dysmorphology Database}

Diagnostic aids such as reference texts and computer systems have arisen due to both the sparsity of expertise and the necessity to cope with the increasing number of dysmorphic syndromes. For database systems, two motivating factors have influenced development: portability and upgradability. The general increase in use of computers, particularly personal computers, has meant that a system designed using well established software is inherently portable and can be readily distributed to medical centres around the world. The only requirements for a consultant to run the system would be a PC and a licence to run the software. This type of system gains an advantage over reference books in that upgradability is easier (and potentially more regular). New versions of the database can be simply distributed to licenced users on disk format. 
The London Dysmorphology Database (LDDB), developed by Winter and Baraitser $[86,89,55]$, was designed with these factors in mind ${ }^{1}$. It is a comprehensive reference facility which holds information about known dysmorphic syndromes and individual cases. The system runs on a personal computer and has been developed using a standard database language ${ }^{2}$. Updates to the database are distributed annually [54].

\subsubsection{LDDB System Goals}

The main aim of LDDB is to provide a comprehensive diagnostic aid. The key to achieving this is to store the necessary diagnostic features for as many of the recognised dysmorphic syndromes as possible. The database currently holds entries for around two and a half thousand non-chromosomal syndromes. Furthermore, syndrome records are linked to corresponding reference entries stored separately within the database. These point to articles from the medical literature concerning the respective syndromes and are intended to encourage the user to refer to other, original research material which might prove valuable in establishing a diagnosis. This helps to put emphasis on the point that LDDB really is a diagnostic aid. Its authors do not make any claims that the system performs diagnosis, rather, very much the opposite. The system is intended for use by specialists in dysmorphology who have the necessary experience to direct the search for potential diagnoses through their own decision making.

A secondary function, and another motivating factor in the design of LDDB, is to provide a look-up facility for rare individual cases. Along with syndrome records, the database also holds entries for affected infants with no recorded diagnosis. Rare individual cases are held in order to facilitate finding a match, or similarity, between two or more such infants. The system does not provide any explicit mechanism for doing this, and it is an investigation left for the human expert to explore. However, the intent is to provide the starting point for such investigation. Subscribers to LDDB are actively encouraged to build up their own patient database by storing in the system details of any infants that they have had contact with, particularly undiagnosed cases. Feedback concerning new cases can therefore be redistributed with new updates to the database. In this way the likelihood of finding a match between individually rare cases, and ultimately in learning

\footnotetext{
${ }^{1}$ The authors of LDDB have recognised that, even with more widespread use of personal computers, a book format still retains some value, and their compendium [85] is actually an off-shoot of LDDB.

${ }^{2}$ Early versions were written in dBaseII. The current version has been developed using CLIPPER.
} 
new syndromes, is increased.

\subsubsection{Architecture}

The operation of LDDB is governed by the manner in which syndromes and cases are represented and stored. Figure 2.1 shows the basic architecture of LDDB. Each recorded

Full Syndrome Name

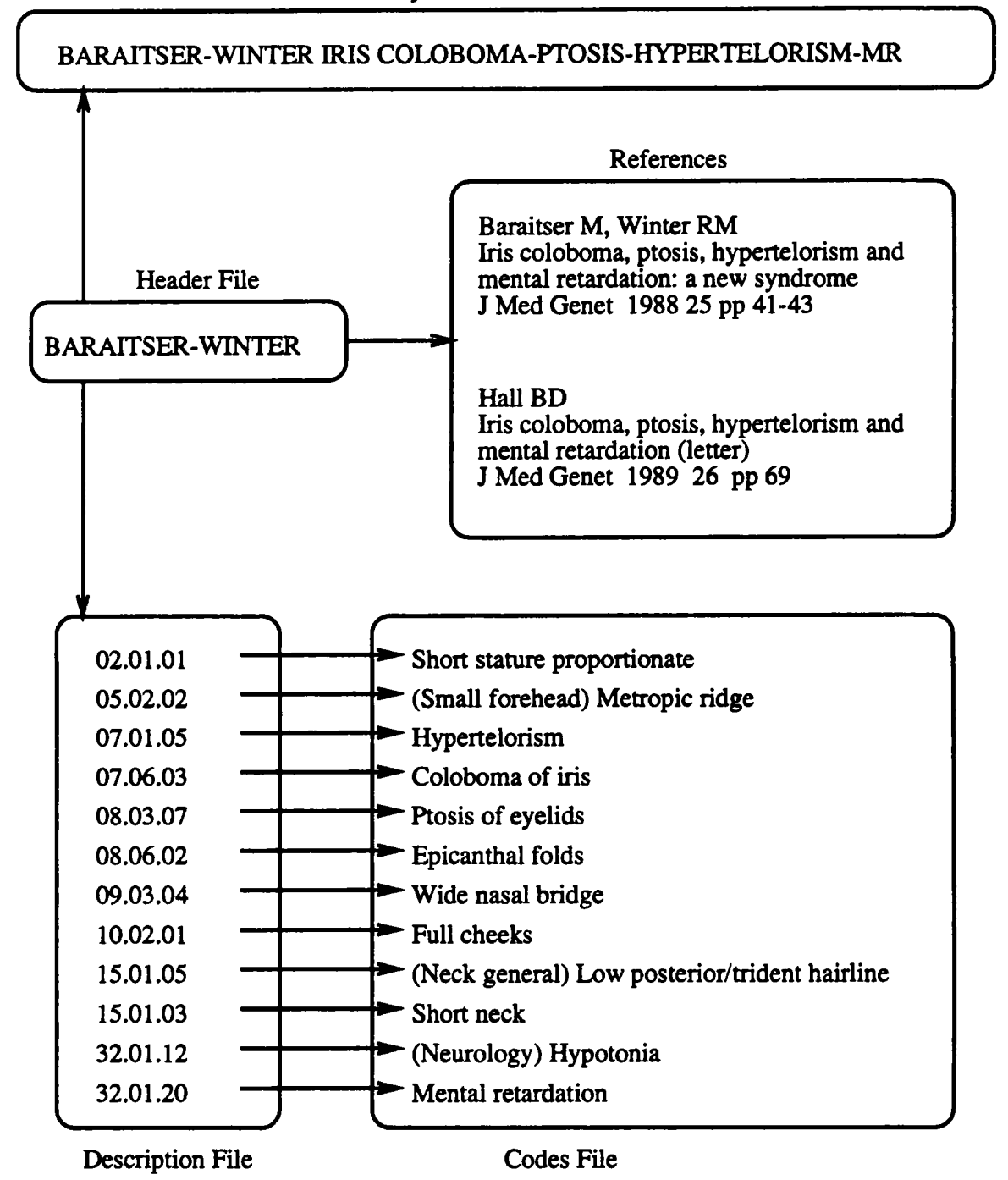

Figure 2.1: The LDDB Architecture.

syndrome, or case, essentially consists of a simple list of dysmorphic features. A header file contains a unique identifier for each syndrome which links to both a description file and a references file (the header file contains a shortened syndrome mnemonic, but links also to a file storing the full syndrome name). The description file lists, for each syndrome 

01. build
13. teeth
25. lower limbs
02. stature
14. voice
26. feet
03. cranium
15. neck
27. blood vessels
04. hair
16. back \& spine
28. endocrine
05. forehead
17. thorax
29. haematology
06. ears
18. abdomen
30. muscles
07. eyes
19. pelvis
31. joints
08 . eye structures
20. genitalia
32. neurology
09. nose
21. urinary system
33. skeletal
10. face
22. upper limbs
34. skin
11. mouth
23. hands
35. gestation/delivery
12. oral region
24. nails

Table 2.1: Clinical regions represented by LDDB.

identifier, a set of numerically coded dysmorphic features. This list may incorporate features of varying diagnosticity (i.e., both principal and occasional features), but has no indication of diagnosticity, or whether a feature is common or rare in a syndrome [89]. Feature codes are translated into textual descriptions via a separate codes file. The numeric coding is central to the operation of the system as it provides the mechanism for search. The codes file consists of an extensive list of possible clinical abnormalities. This master list, which includes over 1200 abnormalities, is subgrouped into thirty five general clinical regions, i.e., organs and parts of the body (see Table 2.1).

Features are arranged in a three-level numeric code. Table 2.2 shows the breakdown of eye anomalies as represented in LDDB. The first level corresponds to the general clinical region (e.g., eyes), the second level refers to a subdivision of that region, (e.g., iris), and the third level refers to a specific abnormality (e.g., coloboma ${ }^{3}$ ).

\subsubsection{Mode of Operation}

The operation of LDDB is based on keyword search, or indexing. The three level dysmorphic feature codes provide the indexes with which the database of syndromes is searched.

\footnotetext{
${ }^{3}$ A lesion or defect of the eye, usually a fissure or cleft.
} 


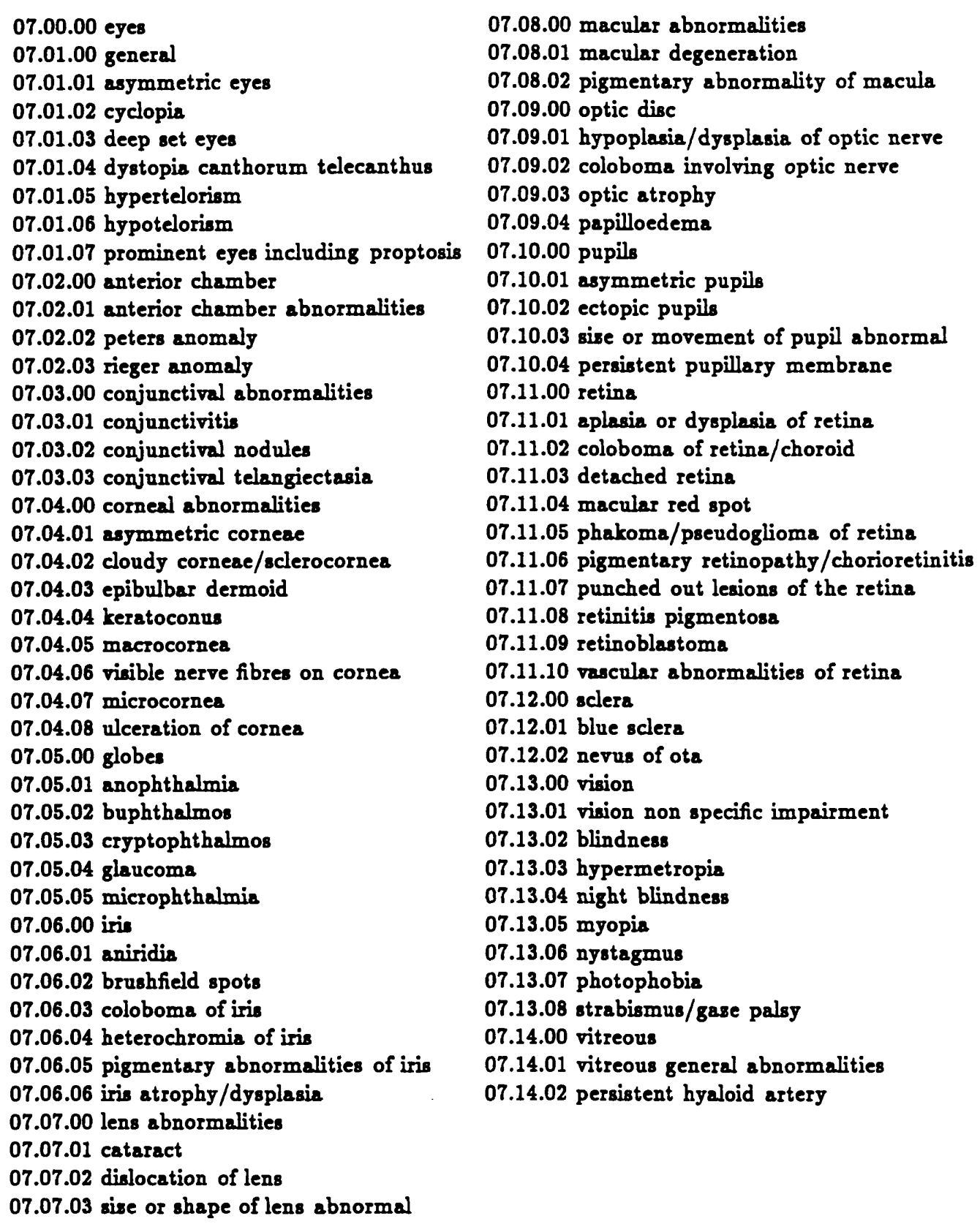

07.08.00 macular abnormalities

07.08.01 macular degeneration

07.08.02 pigmentary abnormality of macula

07.09.00 optic disc

07.09.01 hypoplasia/dysplasia of optic nerve

07.09.02 coloboma involving optic nerve

07.09.03 optic atrophy

07.09.04 papilloedema

07.10 .00 pupils

07.10.01 asymmetric pupils

07.10.02 ectopic pupils

07.10.03 sire or movement of pupil abnormal

07.10.04 persistent pupillary membrane

07.11 .00 retina

07.11 .01 aplasia or dyeplesia of retine

07.11.02 coloboma of retine/choroid

07.11.03 detached retina

07.11.04 macular red spot

07.11.05 phakoma/pseudoglioma of retina

07.11.06 pigmentary retinopathy/chorioretinitis

07.11.07 punched out lesions of the retina

07.11 .08 retinitis pigmentosa

07.11 .09 retinoblastoma

07.11.10 vascular abnormalities of retina

07.12 .00 sclera

07.12.01 blue sclera

07.12.02 nerus of ota

07.13 .00 vision

07.13.01 vision non specific impairment

07.13.02 blindness

07.13.03 hypermetropia

07.13.04 night blindness

07.13.05 myopis

07.13.06 nystagmus

07.13.07 photophobia

07.13.08 strabismus/gase palsy

07.14 .00 vitreous

07.14.01 vitreous general abnormalities

07.14.02 persistent hyaloid artery

Table 2.2: LDDB representation of eye abnormalities. 
1. BARAITSER-WINTER IRIS COLOBOMA-PTOSIS-HYPERTELORISM-MR

2. FRANCOIS [1973] AGEN CORPUS CALLOSUM-COLOBOMA-VERMIS APLASIA

3. FUJIMOTO BRANCHIO-OCULO-FACIAL

4. GARDNER [1983] THROMBOCYTOPENIA-MULTIPLE MALFORMATION

5. HANSON [1976] COLOBOMAS-UNILOBAR LUNGS-AV SEPTUM DEFECT

Table 2.3: Syndromes returned by feature set (07.06.03 AND 15.01 AND 32.01).

The rationale behind the design of the retrieval program is that the user can ask the system for a list of syndromes (or actual patient cases) that contain in their description a combination of specified features. The idea is that the system should be used by an experienced physician who will be able to select the features most likely to lead to a diagnosis and form questions around these. For example, the physician may want to construct a query based on a question such as, "What syndromes can have a cleft palate and either anal atresia or Hirschsprung's disease?" [89]. The system allows the user to construct boolean combinations of features (or rather their respective codes) through a menu driven interface. The authors have tried to emphasise the use of sensible feature combinations in order to return a manageable, short list of possible syndromes. What is deemed sensible is clearly a matter for the human expert to decide.

The authors' of LDDB suggest that good practice is to base a search around one good handle together with a general clinical feature [89]. The ability to search on general features is a useful one because features can show considerable variability among different cases of the same syndrome. This also relates to the problem of variance, or non-uniformity, of feature descriptions (a matter of interpretation) inherent within documented cases. LDDB facilitates the use of general feature search through its three-level representation of abnormalities. The user may enter feature combinations that consist of full (or tight) codes, e.g., 07.06.03, which describe a specific anomaly, and general (or loose) codes, e.g., 15.01, which point to the clinical region and specific subdivision, but not to a specific abnormality. A loose code will match any codes of that particular subdivision, for example, 15.01 will match any codes of type 15.01.??, where ?? corresponds to 00 through 99. The skill then becomes one of finding a useful tightness or looseness to the indexing feature set. Table 2.3 shows the list of syndromes returned using the described technique with an index set of 07.06 .03 (coloboma of iris), 15.01 
(neck, general abnormality) and 32.01 (neurology, general abnormality).

\subsubsection{Discussion}

It should be emphasised that LDDB is very much a tool for use by an experienced dysmorphology specialist. LDDB does not, and is not meant to, make a diagnosis. It does not perform any intelligent matching of entities, it merely 'looks-up' syndromes with specified boolean combinations of features. Diagnosticity of listed features is not defined for individual syndromes. Such information has been avoided due to the fact that it is difficult to assess for many of the less common syndromes where only a few cases have been described. Interpretation of feature diagnosticity is effectively performed by the selection of feature indexes, or handles. Construction of the indexing feature set is thus a crucial step in successful operation of the system. A further ramification is that the list of syndromes returned is not ranked in order of likelihood, or probability of occurrence (in order to do this the system would require some form of similarity assessment based on diagnostic significance). Selected syndromes are returned in alphabetic order.

One criticism of LDDB concerns the inflexibility of the feature representation. The master list corresponds to only one interpretation of how abnormalities may be described. Other specialists may disagree with this master list. This problem is not unique to LDDB. A natural development of the application of computer systems to dysmorphology has been to address this problem of feature representation, but there is no universally accepted schema. When searching with LDDB, if the exact feature required is not apparent, users are forced to select the nearest listed feature that best matches the case finding. To some extent the ability to form loose searches eases this problem. However, when adding to a patient database users are also bound by the Winter-Baraitser representation.

A problem with the representation of syndromes is the lack of certainty with respect to individual features. An abnormality simply exists, or not, within a syndrome description. A problem occurs then if a feature has been found in 90 per cent of cases of a particular syndrome, but not in the object case. Belief about that syndrome is diminished but should not be eliminated. Because LDDB utilises an indexing search method to select candidate syndromes (rather than a general matching method which would allow for some uncertainty) the user must be aware that a syndrome can be omitted unless a relevant index is used.

The success of LDDB is to some extent reliant on its comprehensibility. Its primary 
goal as a diagnostic aid is to allow the user, an experienced physician, to confidently establish a manageable differential. Such confidence will be promoted by a system which is comprehensive, up to date, and universally accepted as an accurate reference source. LDDB has managed to achieve these goals better than most. There are plans to expand the system even further in future versions by adding chromosomal syndromes to the database. Also, a library of clinical photographs has recently been compiled and made available on compact disc format. This contains over 5000 photographs and $\mathbf{x}$-rays which are intended to illustrate the syndromes listed in LDDB.

\subsection{The Skeletal Dysplasia Diagnostician}

The Skeletal Dysplasia Diagnostician ${ }^{4}$ (SDD) is a model-based expert system dedicated to the diagnosis of skeletal dysplasias ${ }^{5}$ which form a group of around 140 syndromes. A dysplasia, by definition, is different from a syndrome (see Appendix A), but for the purpose of this discussion a dysplasia is taken to be a type of malformation syndrome.

\subsubsection{SDD System Goals}

The overall strategy of SDD is to focus towards a diagnosis through consultation with the user. To achieve this, the system incorporates a number of independent bodies of knowledge which correspond to detailed information about individual syndromes, domain specific medical knowledge, and general radiology. Consequently, if sufficient information about the patient is available (input by the user) SDD is designed to formulate an initial differential, process the differential further by generating relevant questions about the patient with respect to the specific syndromes contained within the differential, present these questions to the user, and process the replies in order to assess the active hypotheses. Thus, the intelligent creation of a sensible, refined differential is a system function rather than a human task. A consequence of this is that the system is intended for use by both dysmorphology specialists and general radiologists.

Whilst the specific goal of SDD is to provide a diagnosis, it also aims to provide a relatively generic diagnostic model. The emphasis of this research extends to broader

\footnotetext{
4A co-operation between University College London and Great Ormond Hospital.

${ }^{B}$ Syndromes characterised by abnormal development of bone tissue, which can be generalised or local to specific regions of the skeleton.
} 
issues concerning knowledge engineering, and in particular to knowledge acquisition and knowledge representation.

\subsubsection{Architecture}

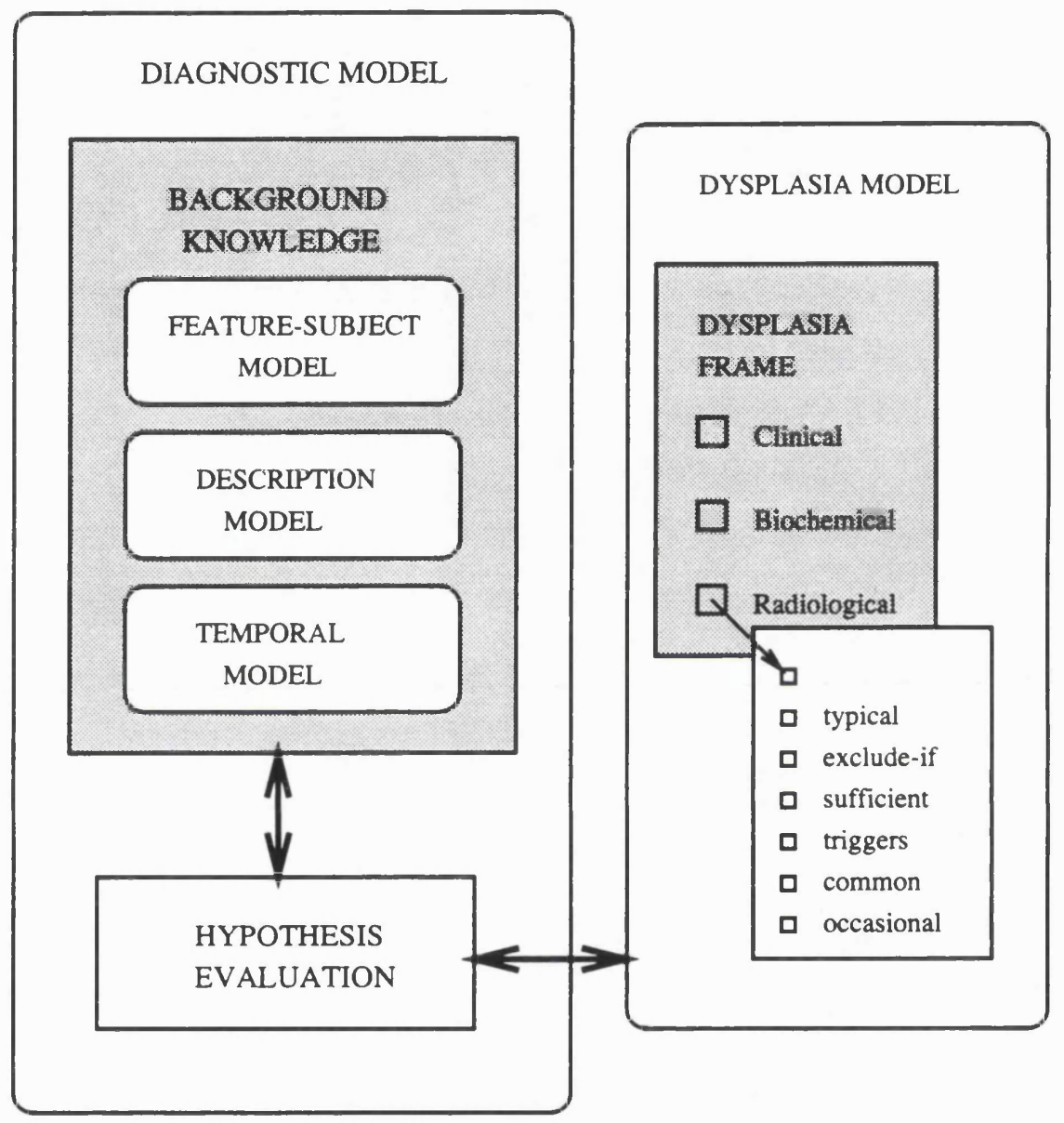

Figure 2.2: The SDD Architecture.

A number of research papers describe the components of the SDD system architecture in detail $[15,36,37,34,35]$. A detailed analysis of all aspects of the system is not considered necessary to this thesis and the following discussion gives a high level overview of SDD.

SDD has been described by the authors as a co-operating architecture [35] in which independent bodies of dedicated knowledge interact during the operation of diagnosis. Figure 2.2 shows an abstracted view of SDD's knowledge components. The dysplasia model is a static knowledge base incorporating a substantial amount of encoded information about each stored syndrome. The diagnostic model constitutes the dynamics of 
the system. It uses the background knowledge-base and controls hypothesis evaluation during the diagnostic cycle.

The dysplasia model represents expert domain knowledge. Such knowledge is facilitated by syndrome frames which have encoded into them six distinct sets of abnormalities of varying diagnostic significance:

1. Typical. These are rare features (less than 1 per cent), but conclusive. Their absence should not count against a possible diagnosis.

2. Exclude-if. Sufficiently diagnostic that they will exclude the dysplasia.

3. Sufficient. A group of features that collectively will strongly indicate the dysplasia (but not sufficient for confirmation).

4. Triggers. There are two subsets: primary triggers, which are highly diagnostic and immediately suggest the dysplasia as a possibility, and secondary triggers, features which are used to discriminate between competing possibilities.

5. Common. Features observed to occur in the majority of cases of the syndrome and thus would be expected to be evident.

6. Occasional. Features which, if they are present, support the hypothesis, but if they are not present do not count against it.

Each set type has a different role to play in the overall diagnostic process. Along with the six categories, features are listed for expected clinical findings, biochemical findings and radiological findings (which are in turn grouped by skeletal regions, e.g., spine, chest and skull).

The background knowledge-base stores foundational radiological, clinical and biochemical knowledge, i.e., expertise that would be expected of a non-specialist physician (some of which is considered to be of a common-sense nature [37]). The background knowledge-base is designed to incorporate three independent bodies of information: a feature-subject model of anatomy and physiology, a description model and a temporal model. For the purpose of this discussion the feature-subject model merits some analysis.

A subject pertains to an anatomical component of the body or skeleton (similar to LDDB's thirty-five clinical regions, but with higher granularity), and an associated feature refers to an abnormality, or condition. The feature-subject model is a rich source of 
information concerning certain types of relationships that occur between feature-subjects. For example, taxonomic information is held such as "dorsal vertebrae are a component-of the thoracic spine which is in turn part-of the spine". Other type of relationships held are linguistic (these are descriptive synonyms for abnormalities and case findings) and inferential ${ }^{6}$ (for example, if the dorsal spine is abnormal then this implies that the spine is abnormal). Relationship knowledge is stored and manipulated using a combination of predicate rules and semantic network structures, and an associated reasoning process.

Within the context of the diagnostic cycle the feature-subject model is responsible for:

- Recognising abnormal and normal findings and contextual information.

- Detecting dependencies and conflicts between listed findings.

- Controlling additional information volunteered during interactive session.

- Organising questions based on findings.

\subsubsection{Mode of Operation}

The first phase of operation creates the initial differential. General information such as patient age and sex is entered followed by the case findings (clinical data and abnormalities exhibited by x-rays). A finding is represented as a subject described by a component-value pair along with a temporal description ${ }^{7}$. For example,

(femora length-long from-3-to-15-years)

(vertebrae height-short from-birth)

In the above examples, femora and vertebrae refer to anatomical subjects, length-long and height-short are component values, and the third components refer to qualitative temporal expressions. The findings are used to identify possible syndromes, or hypotheses. The syndrome database is searched in an indexing mode, but unlike LDDB, SDD syndrome frames have specific indexes against which to search, that is their primary triggers.

\footnotetext{
-An example of a common-sense inference is "if an component is absent then all its parts are absent".

${ }^{7}$ Whilst the mechanics of representation for temporal knowledge is incorporated in SDD the temporal reasoning functionality [34] was not implemented at the time of writing.
} 
The second phase of operation is designed to explore and refine the differential. The system utilises computed ratios [35] between the matching and unmatching anomalies (these are dysmorphic features that are encoded within the syndrome frame that match or negate case findings) in order to rank and cluster the initial differential diagnosis. Clusters are sets of possible hypotheses with highly overlapping expectations, defined by common secondary triggers. Within each cluster, the possible hypotheses are ranked from the

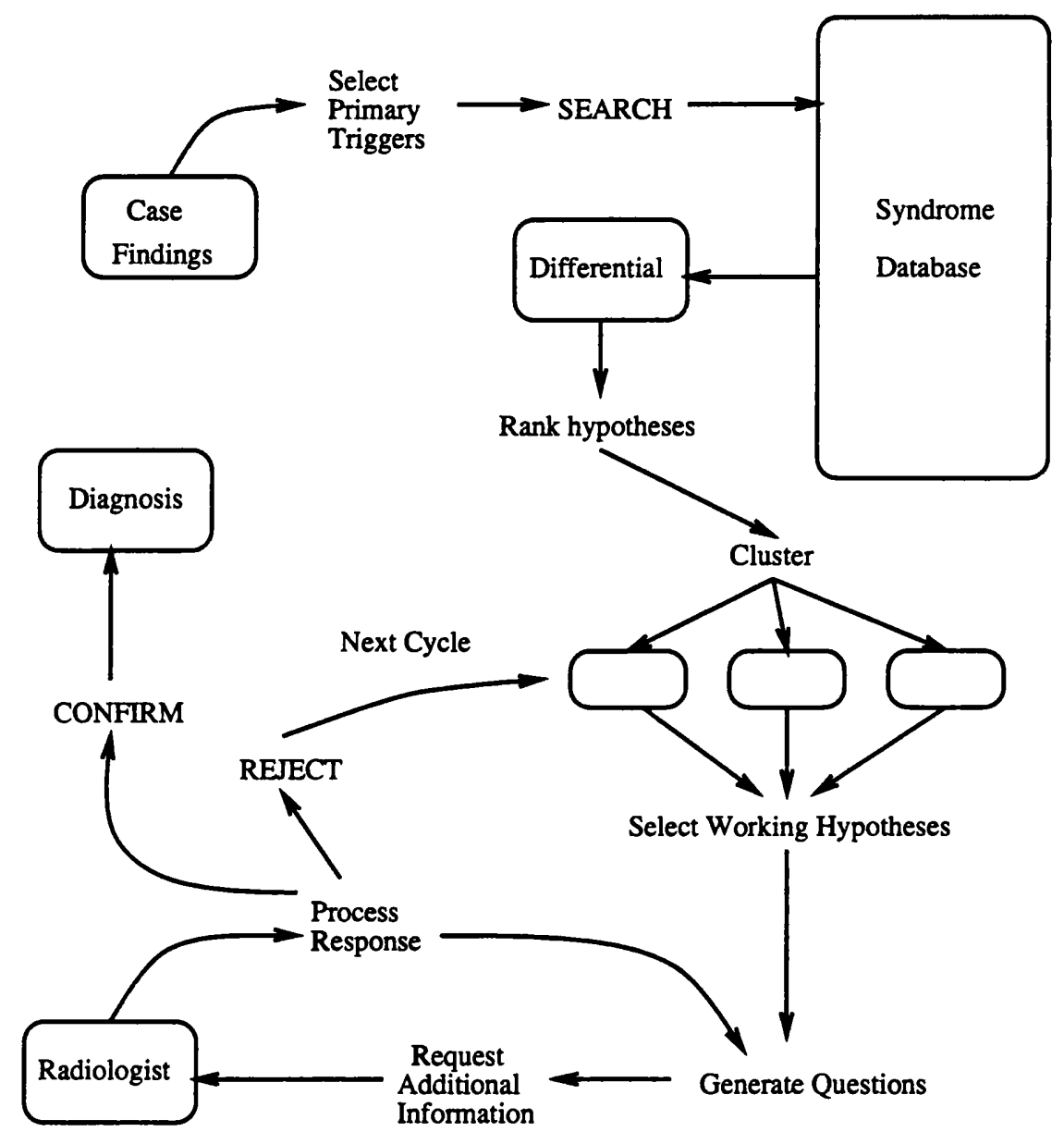

Figure 2.3: The diagnostic cycle of SDD.

perspective of: explanatory power, a ratio between expected features (common features and occasionals encoded within the syndrome frame) that match the case findings, and observations that refute the hypothesis; and sufficient-group match, a ratio between the sufficient set of the syndrome and the corresponding observations. The highest ranked possibilities from each cluster are processed first. A possible hypothesis which matches 
on typical features, or satisfies what is taken to be a reasonable ${ }^{8}$ subset of the current set of hard ${ }^{\theta}$ abnormalities is promoted to a 'working hypothesis'.

The next phase of the diagnostic cycle is a consultation with the physician. The system uses its knowledge of syndrome expectations in conjunction with background knowledge with respect to case findings in order to produce a list of questions, which are put to the user. For example, the working hypothesis may suggest (through knowledge of feature relationships) abnormalities not evident in the findings list. The physician may be asked to indicate whether or not these are present, and may be required to re-examine the $\mathbf{X}$-rays if the relevant information is not at hand. The questions are designed to produce a decision, either in support or against the current working hypothesis. A firm decision may produce a diagnosis. A negation (which ocurs when new evidence counts against the working hypothesis) may result in the demotion of the current working hypothesis back to a possible hypothesis or an exclusion, in which case the syndrome is dropped from further processing. During this final phase the system cycles through all possible hypotheses until a firm decision, and hopefully a diagnosis, is reached.

\subsubsection{Discussion}

SDD offers a very different philosophy, in terms of system goals and design, than that of LDDB. The SDD project is very much involved with research into knowledge engineering (which includes knowledge acquisition and representation, model-based design and temporal reasoning) and the presentation of ideas and results to the $\mathrm{AI}$ community, intermediate goals other than the ultimate aim of producing an accurate diagnostic system for skeletal dysplasias.

SDD is different in scope to LDDB in that it is dedicated to a distinct syndrome group. The tenet in this instance is that sufficient, transferable expertise exists about the group in order to facilitate a feasible knowledge engineering task. Once this premise is accepted, design and development is a matter of eliciting the relevant diagnostic knowledge and compiling this into a working, and accurate model. For instance, knowledge of feature diagnosticity is sufficient within the dysplasia group that six distinct abnormality sets can be defined and collated for all syndromes. This information provides a measure of

\footnotetext{
- An empirically defined numeric score.

'All primary triggers with the exception of specific abnormalities identified through knowledge elicitation.
} 
certainty with respect to features in the syndrome descriptions that does not exist in LDDB.

A problem highlighted earlier with LDDB is that its feature representation is inflexible and does not cater for the variance in interpretation. The user of LDDB can bypass this problem to some extent by using loose indexes. This type of imprecision is dealt with in SDD by knowledge of linguistic relationships (held in the feature-subject model). Knowledge about possible synonyms exists in order to translate findings to a common representation that can map case findings to features encoded within the dysplasia frames.

One of the most important differences between LDDB and SDD is that SDD performs similarity assessment in order to refine the differential, and ultimately propose a diagnosis. To achieve this, there is some reliance on numeric ratios (i.e., sufficiency set and explanatory power). A numerical approach is common (see Section 2.4), but not ideal. Experts find it difficult to agree on a definitive relationship between diagnostic weight and frequency of occurrence (the example of mental retardation cited earlier demonstrates this problem), and do not comfortably express abnormalities in terms of numeric values. However, designing a similarity metric on purely qualitative measures would be difficult given the inexact nature of the expertise. The use of numerical measures for diagnostic power is a compromise if such knowledge is readily available. A strength of SDD is that numeric comparison is merely part of the overall hypothesis evaluation, whereas in other systems it constitutes the entire similarity assessment.

Another distinction between SDD and LDDB is that the latter stores undiagnosed cases. SDD does not store cases at all. This suggests a difference in the overall objectives of the two systems. SDD operates within a strong domain theory. That is to say, a relatively well understood branch of dysmorphology. Knowledge of skeletal dysplasias is sufficiently complete that a database of detailed prototypical ${ }^{10}$ syndrome frames may be compiled. Its aim is diagnosis with respect to this fairly static knowledge. The goals of LDDB are broader and weaker. It aims to provide an initial differential across a large spectrum of syndromes. Undiagnosed cases are stored within LDDB to provide some focus on learning more about the domain.

The SDD project is ongoing, and further work is intended to extend the system to include an image database comprising $x$-rays relating to the relevant skeletal dysplasias.

\footnotetext{
${ }^{10}$ The syndrome records of LDDB may also be described as a list of prototypical features, but without the indication of diagnostic power that is held in SDD
} 


\subsection{Related Work}

There are a number of independent systems dedicated to some aspect of dysmorphology. The following section provides an overview of this work. The two systems described above have been selected in order to demonstrate the diversity in design. The systems described below overlap in many aspects, both with each other and the two aforementioned systems. These are discussed with reference to any significant differences in approach.

\subsubsection{On-Line Databases}

The Birth Defects Information Service [13] was one of the earliest information retrieval type applications specific to malformation syndromes. The original development of BDIS involved an on-line database accessible via modem link. A later version (Micro BDIS) is based on a personal computer [14]. A text specific to inherited diseases, McKusick's Mendelian Inheritance in Man [51], has been accessible as an on-line database since 1987 (On-Line Mendelian Inheritance in Man, OMIM [67]).

These systems perform a very similar function to LDDB, i.e., provide a centralised reference source designed with a view to regular updates [19]. As with LDDB, a differential is obtained through a user selected index set, but BDIS also provides a ranking algorithm in order to grade the candidate hypotheses. This is facilitated through diagnostic weights assigned (selectively) to features according to frequency and specificity. A score is summed for each candidate based on the overlapping features with the case and the associated weights. With the same misgivings as the developers of LDDB perhaps, the researchers involved with BDIS have stressed the limitations of the ranking algorithm. It is intended to assist the user only in narrowing the range of possibilities rather than suggesting the most likely diagnosis. Furthermore, the authors have indicated the difficulties in assigning subjective weights to features based on frequency.

\subsubsection{SDR}

The Skeletal Dysplasia Registry (SDR) is a recent development designed specifically for diagnosis and research of skeletal dysplasias ${ }^{11}$. It has comparable architecture to that of LDDB (designed using PC based DBMS software) and a similar mode of operation (boolean search based on abnormal features). As with LDDB, this system is intended for

\footnotetext{
${ }^{11}$ Developed by D.L. Rimoin and co-workers at the Cedars-Sinai Medical Center, UCLA.
} 
world-wide distribution, and, perhaps even more so than LDDB, there is an emphasis on research of unknown syndromes. SDR contains a database of classic skeletal dysplasias (overlapping with the 140 or so that SDD covers), and a large patient database of diagnosed and undiagnosed cases (over 4000) compiled from many institutions around the world. Whilst LDDB accounts for many more syndromes, SDR provides very comprehensive details concerning each case such as the referring physician and their affiliated hospitals.

\subsubsection{El Busca}

El Busca ${ }^{12}$ is a database application with a reference file of two hundred syndromes and a master list of 175 abnormal features [64]. A feature of this work that overlaps with BDIS is that abnormalities are assigned an associated diagnostic weight expressed as a direct function of frequency. Each feature is weighted by the difference between the number of syndromes including that feature and the total number of syndromes in the reference file. Therefore, the weight of each abnormality is inversely related to its frequency amongst the syndromes, and is meant to express its predictive value. Thus, a feature that occurs in only five of the two hundred syndromes is considered, in terms of frequency, highly predictive. System operation employs a standard boolean search and up to fifteen features may be entered. The system then selects candidates having three or more of these features. The candidates are then ranked according to an absolute value, resulting from adding the weights of those features of the case that overlap with the syndrome. The top twelve hypotheses are then presented to the physician. In tests, success was defined by the inclusion of the correct diagnosis in the top twelve ranked hypotheses.

\subsubsection{SYNDROC}

The SYNDROC program developed by Schorderet is one of two systems that have utilised a Bayesian formula in order to rank a differential diagnosis $[66,68]$. SYNDROC has a smaller, but similar syndrome database structure to that of LDDB (initial research was conducted with a database of around 300 syndromes which was later expanded to over 1000 , including a small percentage of chromosomal syndromes), and includes a similar

\footnotetext{
${ }^{12} \mathrm{El}$ Busca has not been adopted as a diagnostic tool and has not been developed any further.
} 
master list of abnormal features, called signs. The system can store cases in very much the same way as LDDB, with essentially the same goal in mind. Furthermore, as with LDDB, SYNDROC allows boolean combinations of features to be selected by the user (no diagnostic weights are provided) in order to probe the database. The main difference from LDDB, and similar databases, is that an attempt has been made with SYNDROC to rank the differential in order of probability. To achieve this the system has employed a modified version of Bayes' formula (Equation 2.1 shows the correct definition of Bayes' rule).

$$
P(D / F)=\frac{P(F / D) \times P(D)}{P(F)}
$$

Bayes' rule states that the probability $P(D / F)$ that a patient has a disease, given the presence of a particular set of findings, is equal to the probability $P(F / D)$ of the occurrence of this set of findings given the disease, multiplied by the frequency $P(D)$ of that disease in the population and divided by the incidence $P(F)$ of the findings in the general population [12]. Bayes' theorem makes certain assumptions, however, which make its applicability to dysmorphology questionable. The disease set is assumed to be exhaustive $\left(\sum P\left(D_{i}\right)=1\right)$ and exclusive (i.e., only one disease is present). Additionally, the formula assumes that all signs are independent of each other. In practice, the parameters of the formula will only be partially known, especially for the less common syndromes. To cater for this, Schorderet employed a subjective value instead of $P(F / D)$, and calculated $P(F)$ from the database (the program is thus called pseudo-Bayesian).

Once the patient findings have been entered, SYNDROC calculates a pseudo-Bayesian probability (called a certainty coefficient which ranges from 0 to 1 ) against every syndrome held within the database. The differential diagnosis consists of those syndromes lying between the highest score and a lower limit of half of this highest score. Initial results using 100 firmly diagnosed test cases (against a database of around 300 syndromes) showed a high level of performance (over 90 per cent agreement with a medical geneticist). However, as the database grew the authors have indicated that the estimation of $P(F / D)$ became increasingly difficult and system performance decreased.

\subsubsection{Weiner}

Weiner et al. has also used a pseudo-Bayesian formula to rank the differential diagnosis [82, 81]. As with LDDB and SYNDROC, the database contains patient records along with around 250 syndromes. There are some differences to both LDDB and SYNDROC, 
however. In this model, diagnostic value is indicated for syndrome features. Four levels are defined: essential, prevalent (features that occur in the majority of diagnosed cases), occasional, and rare. Features are coded to a master list as before, but in this instance there is no layering (thus, no loosening of feature sets is possible).

The modified Bayesian formula used by Weiner et al. is described below. As with SYNDROC it is applied exhaustively, but in this instance it is applied twice: once to calculate a probability for, or in support of the syndrome $\left(P\left(F_{j} / D_{i}\right)\right)$, based on case findings $(j)$ and syndrome features that match (where $i$ denotes an individual syndrome), and again to calculate a probability against the syndrome $\left(P\left(F_{j} /-D_{i}\right)\right)$, based on syndrome features that are absent in the case findings. The likelihood $\left(l_{i j}\right)$ of a feature for a given syndrome is denoted by Equation 2.2 .

$$
l_{i j}=\frac{P\left(F_{j} / D_{i}\right)}{P\left(F_{j} /-D_{i}\right)}
$$

The overall likelihood $(L)$ of the disease $(D$ vs. $-D)$ is then denoted by the product of the individual feature likelihoods (Equation 2.3),

$$
L_{i}=\prod_{j} l_{j}\left(\frac{D_{i}}{-D_{i}}\right)
$$

and the syndrome probability is then given by Equation 2.4 .

$$
P\left(D_{i} / F\right)=\frac{L_{i}}{\left(L_{i}+1\right)}
$$

A syndrome scoring a probability of 0.85 or greater is included in the differential. To achieve greater accuracy, the system contains a table of numeric values to account for the diagnostic significance of features. These are defined according to the diagnostic level (essential, prevalent, occasional and rare) and whether the match is for the syndrome or against it. The table is used to increase or decrease the relative contribution of each feature (by a factor ranging between 0 and 1 ) with respect to the overall probability score for a syndrome. For example, if an essential feature (which is defined for a syndrome) is not evident in the case findings then its contribution will by multiplied by a factor of zero, thus resulting in an overall probability of zero, hence ruling out the syndrome. If a feature is defined as an occasional for a syndrome, and the calculation is in support of the syndrome $(P(F / D))$, the multiplying factor can be relatively high if it exists. If the calculation is against the syndrome $(P(F /-D))$, and the feature does not exist, then the multiplying factor should be relatively low. This demonstrates the fact that occasionals 
can add support to a diagnosis if they exist, but should not count against a match if they don't.

Results from this work have been documented in which 49 test cases were used, with 34 having no recorded diagnosis [81]. For the 15 known cases there was agreement with a human expert. In 15 of the 34 unknown cases the system did not reach a diagnosis. Of the other 19 cases the system indicated a differential which proved instrumental in finding a correct diagnosis (that is to say, it is implied that further investigation lead to confirmation of a syndrome listed in the differential).

High accuracy has been reported for both SYNDROC and the work by Weiner and researchers. However, these results were based on experiments that incorporated a relatively small database and distinct test set (i.e., test cases ranged across non-related syndromes). Schorderet has indicated that an increase in the number of potential syndromes has a detrimental effect on system performance [66]. It would also appear that a practical application of a Bayesian formula to the dysmorphology field is questionable given the constraints of the theory and the sparseness of data.

It is interesting that although a boolean search capability is apparently available in both these systems, there is no comparison between the differential diagnoses derived by the pseudo-Bayesian methods and those arrived at through an indexing method as in LDDB. Instead, there has been a preference to apply the pseudo-Bayesian equation exhaustively across the database.

\subsubsection{GENDIAG}

In many ways, the GENDIAG program [23] is comparable to both SYNDROC and Weiner's system. It incorporates a database of syndromes and employs a computed probability theorem to rank the differential. In this instance the ranking formula is based on belief functions. As before, this work follows the assumption that a syndrome may be represented as a list of characteristic signs that have an associated indication of their frequency within the disorder. In order to apply the discriminating metric with accuracy, the program is, therefore, limited to the more common syndromes for which this statistical information is available.

GENDIAG has some important similarities to LDDB. It utilises a master list of features against which the expert user is obliged to represent the new patient. However, unlike LDDB, this master list indicates a diagnostic weight ranging from 3 (which is 
equivalent to a hard feature) to 1 ( the level of least significance). A second overlap with LDDB is that GENDIAG allows flexible mapping of case features to syndrome features through generalisation. This facility serves the same purpose as the layered coding representation in LDDB. In GENDIAG, equivalent feature descriptions are tied together within tree-like structures. For example, a very general feature description such as malformed ear will be linked to increasingly more specific anomalies such as malformed concha, malformed helix and flattened ear folds (which is the most specific). Finally, GENDIAG also stores relevant journal references within its database.

The mode of operation of GENDIAG is not unlike what has been described previously. The characteristic signs of a new case are identified and entered by an expert. The case features that, according to the master list, have a diagnostic weight of 3 are used to probe the syndrome database and return a differential diagnosis which is in turn ranked by the computed belief function of each hypothesis.

\subsubsection{Preus}

Although Preus et al. have not developed a diagnostic system as such, they have offered an alternative view towards syndrome classification through numerical methods $[57,58]$. The premise in using such techniques is that they will facilitate an unbiased (objective) categorisation of cases and confirm strong phenotype-karyotype correlations.

The main principle involved with this work is to generate clusters of cases across a multidimensional feature space. Individual cases are compared against all other cases along all possible features to form a multidimensional matrix, or array. This array then corresponds to a combinatorial mapping between the whole data set. For example, one study involved 75 cases with a master list of 178 possible abnormalities (all features noted across all 75 cases). The corresponding similarity matrix would have $75 \times 75 \times 178$ cells. Each cell of the matrix stores an associated value, or score, that represents the similarity between two different cases for one specific anomaly. Where cases do not match on a feature the associated cell will be empty or have a nil value. A numerical formula ${ }^{13}$ is then applied to the matrix in order to define boundaries between clusters of similar cases across multidimensional space. Each cluster will then hopefully represent a discrete class of syndrome.

\footnotetext{
${ }^{13}$ Preus has experimented with a number of numeric formulae, e.g., furthest neighbour and group average.
} 
The experimental approach has been to select a data set comprising of cases across a small number (2-4) of known syndromes allowing direct evaluation of the resulting clusters. To a great extent the same criticism as those aforementioned systems employing probability based formulas is valid here. The statistical information required for this approach is really only available in sufficient detail for common syndromes. With this work there is no diagnostic system, rather a statistical confirmation that cases form groups along phenotypes and a possible procedure for calculating a confidence level in a diagnosis (as with previous ranking formulae).

\subsubsection{GS.52}

Gierl and co-workers have developed an expert system [21] with a high level architecture similar to SDD (though not specific to one syndrome group). There exists a body of metaknowledge which controls a consultation with the physician (and which is comparable to the background knowledge model of SDD). The database stores patient data along with around 230 syndrome prototypes (which is similar in design to the dysplasia model of SDD, although SDD does not hold individual cases). A third part of the system incorporates a master list of about 800 signs along with syndrome-specific rules (cf., SDD's feature-subject model). Three system operations have been identified: patient data management, consultation, and knowledge acquisition. All new cases are stored in the database with respect to the master list of features. A diagnostic process is initiated through a consultation. An individual case is chosen from the database. The physician then selects an initial differential based on the listed signs (an automated search mechanism is apparently available, but this has not been described in the literature). A refining procedure then follows. The physician is given a choice regarding the mode of operation of GS.52. The user can apply the relevant syndrome rules to derive the most likely diagnosis. Alternatively, for relatively common disorders, GS.52 is another system that allows the differential to be ranked according to probability formulae $[77,63]$. A solely human assessment is also indicated by the authors as an alternative possibility. The final decision regarding the diagnosis is left to the expert.

A major thrust of this work has been the knowledge acquisition model. Specifically, in developing a semi-automated method to generate the syndrome prototypes. The syndrome prototypes are derived from the patient database using a combination of human guidance with respect to the use of a statistical formula [21], employed to calculate the 
diagnostic weight of individual features. Thus, whereas the database of prototypes is comparable to that of SDD and GENDIAG, the encoded significance of the listed anomalies is self-generated (GENDIAG and SDD elicited this knowledge from domain experts).

\subsubsection{DYSMOR}

The DYSMOR system, developed by Veloso et al. $[79,80]$, uses conditional rules in order to refine a differential diagnosis. As with LDDB, its database stores around 750 nonchromosomal syndromes, individual cases and journal references, and it utilises a master list of some 1400 signs.

The mode of operation is to input the features of a new case, select a differential list of hypotheses, and then allow the system to eliminate candidates by employing its expert knowledge. A frame representation is employed for syndrome records (cf., SDD). Incorporated into each syndrome frame is a list of expected abnormalities, and an associated body of conditional rules. For each candidate the associated rule-set is invoked in order to promote or eliminate it (e.g., syndrome $x$ if all the findings true). The rules drive a consultation during which the user may be required to confirm the existence, or absence, of certain specific anomalies. As with SDD the consultant may be required to re-examine the patient.

An interesting aspect of DYSMOR is that, whilst it does utilise diagnostic weights, significance is not stored with reference to individual signs. Instead a confidence score is attributed to each syndrome rule. However, as many of the rules are associated with specific anomalies, diagnostic weights are used in effectively in the same manner as in other systems, albeit indirectly.

\subsubsection{Visual Reference Systems}

Visual reference is useful in all aspects of diagnosis (e.g., radiology and chromosome analysis), and therefore it is not surprising that workers involved with both LDDB and SDD intend to incorporate an image database in future versions of their respective systems. To date, a couple of visual reference databases have been developed. Yamamoto et al. developed a syndrome database with associated pictorial images for each database entry $[91,90]$. Around 300 syndromes and associated pictures were compiled from standard texts such as Smith's compendium [30] and Goodman's The Malformed Infant and Child [22], along with specialist journals. Images were digitised and stored using an image 
scanner. A more recent innovation is demonstrated by the POSSUM system (Pictures of Standard Syndromes and Undiagnosed Malformations [7, 6, 72]) and its complement OSSUM (dedicated to the skeletal dysplasias group). These databases were developed using an interactive video recorder, storing digitised images (photographs of some 1500 patients) on an optical disk. Views include clinical photographs of the head, face, hands, feet etc., along with associated radiographic images. The operation of both these systems follows the boolean indexing approach, and like LDDB the POSSUM system has regular updates distributed on disk ${ }^{14}$.

Apart from the clear assistance it may give in diagnosis, a secondary advantage of visual reference is that it can ease the inherent problems with nomenclature and terminology. POSSUM incorporates images with its dictionary of traits (equivalent to LDDB's master list of feature descriptions), to which a physician can refer if in doubt about the interpretation, or description, of a specific anomaly [8]. This can generate a degree of consistency when new cases are added to the database, but as with LDDB, the user may feel constrained by the system definitions.

\subsubsection{Discussion}

Each of the systems described above may be classified with respect to three general categories: database systems, ranking systems, and expert systems. A fourth sub-category which may intersect any of the three main groups is visual systems. As can be seen from Figure 2.4, Ranking Systems are those that lie somewhere between a pure database application and an intelligent system (but by default are at least databases). Within this group, some have employed diagnostic weights and an associated summing algorithm to grade hypotheses, whereas others have adopted a probabilistic approach. There is inadequate data for a quantitative assessment of the relative performance of each of the systems mentioned, though some qualitative comparisons can be made. Dysmorphology database systems are designed with an emphasis on supplementary study of the literature. They will always produce a manageable differential with guidance from the physician. Ranking systems have managed high accuracy, but this measure is usually adjudged on whether or not the correct syndrome is present within the highest ranked section of the differential.

\footnotetext{
${ }^{14}$ Since the recent availability of the Dysmorphology Photo Library which is associated with LDDB, POSSUM and LDDB overlap in most aspects of use. Dr. Winter has indicated that POSSUM and LDDB carry equal stature, and a case study of POSSUM could have been used as an alternative to LDDB [88].
} 


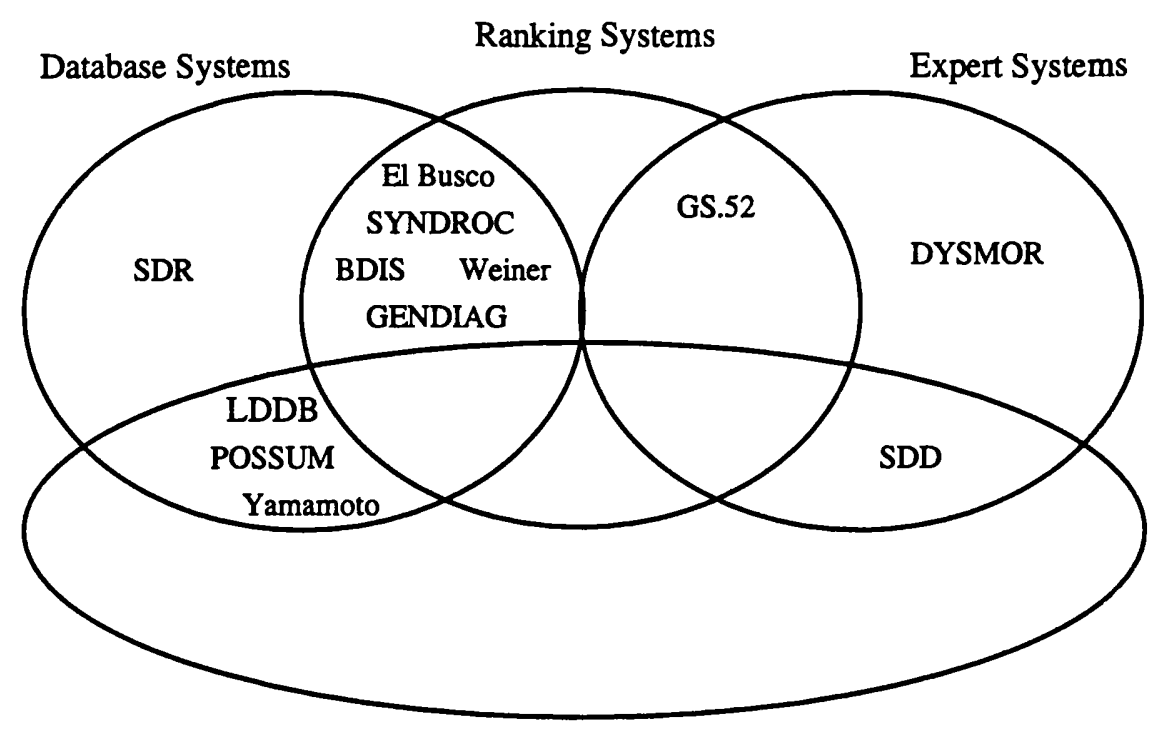

Visual Systems

Figure 2.4: Syndrome computer systems by category.

Furthermore, these results have evolved from experiments with common syndromes (the strategy of ranking a differential through probability has been noted as inappropriate for less common conditions [55]). It has not been demonstrated by how much these systems increase performance when compared to standard databases.

Similarly, it is uncertain for the moment how much improvement will be achieved by knowledge engineered systems in accurately refining a differential diagnosis, or proposing correct diagnoses. However, given the scope of a system such as SDD, which is dedicated to a single syndrome group and has involved radiology specialists throughout its development, significant accuracy can be expected. Perhaps one gain that expert systems will have is in their potential as teaching aids. As they effectively attempt to model diagnostic procedures, they may prove valuable in training students or general medical practitioners.

There are clear trade-offs between approaches. Ranking systems may increase performance slightly when applied to common syndromes for which sufficient statistical information is available, but suffer in scope compared to comprehensive databases such as LDDB, SDR and POSSUM (if a ranking system did store a comparable number of syndromes the ranking procedures involving probability formulae would still only be feasible for the common disorders). This is true also of expert systems. There is also a trade-off 
between ranking systems that use a probability formula to grade hypotheses, but which may not have a clear mapping to real world procedures, against a knowledge engineering approach which more accurately models the diagnostic process. The development cycle and cost of a knowledge-based system are significantly greater. This factor must be weighed against the ultimate performance when compared to the shorter development cycle of a ranking system that employs a 'plug-in' formula.

All the above systems have one aspect in common, they are all static systems designed primarily for diagnosis. That is to say, each system stores stable syndrome entities which are not updated during the diagnostic procedure. Furthermore, any new disorders are simply added to the database by the administrator. The recognition of new disorders is sometimes facilitated by the inclusion of case records, but detection of similarities between such cases is a human activity. In conclusion, no automated assistance is provided for the revision of known syndromes, or for establishing new syndromes ${ }^{15}$. However, this aspect of dysmorphology is clearly a very significant part of the field. Much effort is given to correlating patient data in order to derive a greater understanding of known conditions and establish new syndromes. This dilemma provides the stimulus for this work. The learning aspect of dysmorphology gives scope for a new contribution to the field. This thesis describes the development of a system that incorporates a dynamic database architecture intended to facilitate, to a greater extent, the learning aspect of dysmorphology.

\subsection{Modelling Procedures and Aspects of Dysmorphology}

A higher order objective of some computer systems dedicated to medical fields is to model some aspect of expert procedures. In a relatively complex medical domain, such as dysmorphology, there exist clear trade-offs at the outset of system design and development. The systems that have been described in this chapter demonstrate alternative research goals. Although the London Dysmorphology Database project has made no explicit attempt to model diagnostic procedures, its basic mode of operation (i.e., selection of indexes) does implicitly provide a minimal diagnostic model. The Skeletal Dysplasias Diagnostician project, on the other hand, has spent significant effort in designing its

\footnotetext{
${ }^{15}$ The GS.52 system has employed a semi-automated approach in order to calculate diagnosticity within its syndrome records. Here the physician applies a probability formula across diagnosed cases.
} 
diagnostic model through knowledge acquisition sessions with specialists. Ranking systems such as SYNDROC demonstrate a compromise solution. Whilst no great effort is spent in acquiring expert knowledge, an attempt has been made to increase the assistance available to the physician, not so much by modelling diagnostic processes, rather by replacing them with a readily available method.

The work described in this report has not attempted to acquire detailed procedural diagnostic knowledge. At the outset it was not considered feasible to derive an application using rule-based or model-based techniques. However, this work has aimed to apply relevant artificial intelligence techniques to appropriate aspects of dysmorphology. A higher order objective has been to address the learning aspect of dysmorphology. This has in turn directed the research to the interdependent problems of modelling similarity assessment, representation of syndromes and patient cases, and the architecture of the database (or case-base) in which they are stored. These problems (and the solutions provided by this research) have been mapped to research in Case-Based Reasoning. One of the basic goals in addressing the learning task of dysmorphologists is in some way to assist this process through automation, i.e., in modelling, to some degree, the learning process. To this end the work is also mapped to research involving incremental concept formation techniques, which in many ways overlap with case-based learning. The following sections give some background information on these topics.

\subsection{Case-Based Reasoning and Learning}

\subsubsection{Introduction}

Case-Based Reasoning (CBR) has, over recent years, grown in popularity as a model within artificial intelligence (AI) and knowledge engineering. There is now a wealth of designated CBR literature for both general reference $[1,60,41,46,45]$ and research $[97,39,40,98]$. For this reason detailed analysis of CBR is deemed inappropriate in this thesis. The following overview discusses the main principles of CBR that have relevance to a CBR model in dysmorphology.

A number of attractive features of the CBR model have been instrumental in its proliferation when compared to first generation expert systems [33]. This section considers a number of issues which have relevance to the designated domain of this research, and for which it is thought that CBR may provide a step forward. 
An important limitation of rule-based and model-based systems is that the knowledgebase is comprised of static knowledge. Although artificial intelligence has many definitions, a common perspective is that knowledge should be self-perpetuating, i.e., an intelligent system should be able to learn through its own mechanics. Another important factor follows from the cognitive influences on AI. Do the computerised representations of knowledge employed to date (production rules, semantic networks, frames etc.,) allow an acceptable mapping of human cognitive processes? Finding a valid cognitive representation of knowledge is an avenue pursued by some researchers in artificial intelligence. Ideas from CBR do, to some extent, address these issues. In a case-based reasoning system, knowledge is comprised literally of cases. Whatever the application domain, the knowledge stored consists of past cases, exemplars, or precedents. One assertion that is popular with the CBR community is that this representation of knowledge presents a valid representation of human memory. In CBR, problem situations are solved with reference to these stored past experiences. This is seen in every-day life, and particularly in domains such as law and medicine (often cited as the classic domains of CBR). Utilisation of experience in the guise of past cases has already been shown to be an important aspect of dysmorphology. The two major tasks of diagnosis and learning new syndromes are performed through recognition and reference to previous diagnosed or undiagnosed patients. Furthermore, one of the key features of CBR is that its knowledge, in the form of its case memory, or case-base, may be automatically updated. The design of an architecture for an evolving, or updatable case-base, by Schank (reported in his landmark book Dynamic Memory [65]) and the subsequent work by a number of his students (most notably Kolodner $[42,43])$ in many ways laid the foundations for CBR research.

Another aspect that is important to the mechanics of some case-based reasoning systems, is that suitably similar cases may become merged through generalisation. That is to say, a number of similar past cases may be automatically grouped together under a more general superclass, or prototype case. In order to achieve this, the system must therefore be able to recognise similarity. This is essentially how in the real world of dysmorphology a recognised syndrome evolves. An important facet of CBR theory is introduced here; a case memory is structured, or organised such that similar past cases are stored in proximity to each other. The key to CBR operation is recognition of (or remembering) previous examples and mapping the new case (or patient) to a previous case or generalised case (or syndrome). This mapping is achieved by confined search 
through the organised case-base.

Another problem with early knowledge-based systems is that they always work from first principles. Even if the new problem situation (or case) has been encountered previously, a rule-based or model-based system will still calculate its solution by proceeding from its designated functional starting point. An important belief amongst researchers in CBR is that past cases can, to a great extent, provide a solution or part solution in a packaged format. Thus, laborious recalculation from first principles may be avoided. The key to achieving this is to recognise the relevant past case. This characteristic is also reflected in dysmorphology, and medical practice in general. Winter and Baraitser describe one possible diagnostic process as gestalt recognition [85], when an experienced physician may immediately think of the possible diagnosis when looking at a case (triggered by a the existence of a very significant anomaly). The physician is certainly not working from first principles in this instance. However, an inexperienced physician may be forced to work from first principles. It is essentially a matter of experience (knowledge and retrieval of past cases).

A further, but by no means less important problem with early expert systems, is the knowledge acquisition bottleneck. Knowledge elicitation from a domain expert is a major task of knowledge engineering. It is possible that the knowledge engineer may force the embodyment of knowledge into a format which may not be a natural representation for the expert in a field where there is much uncertainty and impreciseness. Dysmorphology provides a good example of this dilemma. There is so much background medical knowledge required for diagnosis that much of it can be passed by if it is not specifically prompted for by the knowledge engineer. A benefit of CBR, is how it can ease knowledge acquisition. As cases are regarded as a natural representation for experiencebased knowledge, exemplar cases, or precedents, may provide a natural format by which knowledge may be transferred. However, this aspect will not completely satisfy the demands of dysmorphology. Whilst prototype syndrome cases may have a sufficient level of knowledge encoded within them for a database, a system attempting to develop a strong diagnostic model will require further background medical knowledge.

\subsubsection{A General CBR Model}

Two styles of CBR have evolved: problem-solving [3, 2, 26, 25, 47, 48, 74, 73] and classification $[4,5,56]$. The basic difference between them is that in a problem-solving domain 
a retrieved past case will be adapted to fit the new problem situation, whilst in a classification domain adaptation of past cases is not required. For example, in mathematics a problem may be solved through recognising a previous example which posed a similar set of circumstances. The solution of the previous example may be used, and transformed to fit the new problem. A simple type of transformation would be to change the parameters of an equation. Classification CBR is applicable in domains where a solution is a matter of interpretation, and previously classified examples are used only for comparison. In dysmorphology, a past case is used in this way to help classify (or diagnose) the new patient. The referent case which is retrieved from memory is not actually altered in any way. The diagnosis (or solution) is facilitated implicitly through assessment of similarity (adapting the description of the referent case would not make sense in this instance). Consequently there is a subtle difference in the way that the style of CBR affects learning. In a problem-solving system, the adapted solution is what is stored. Thus, knowledge is increased as the system can account for a greater number of problems. A classifying system merely stores the new case. However, the location at which it is stored (i.e., the proximity of the new case with respect to a syndrome prototype) effectively implies the solution. Learning then occurs through localised reorganisation of memory where the case is stored (as category information is increased with each new stored case). Thus, the organisation of the case-base is crucial in a classification system. For a problem-solving system, however, a possible solution is not necessarily defined by its location in memory. The only requirement is that a precedent may be (efficiently) retrieved given a set of indexes ${ }^{16}$.

Figure 2.5 illustrates an abstracted representation of CBR which accounts for both styles of reasoning, and the procedures of computer assisted diagnosis reviewed earlier in the chapter. The first stage of CBR is to identify salient features of the new case that may serve as indexes with which to probe case memory. This serves a similar purpose to the selection of a boolean feature set in LDDB ( a human function), or the identification of primary triggers in SDD (a system function). These operations enable a search through the database, or case-base, and the retrieval of multiple objects, i.e., syndrome prototypes or individual cases. The next phase involves refining the retrieved list of past cases. This will tend to be more important for a problem-solving system, as further processing (which

\footnotetext{
${ }^{16}$ If a problem-solving CBR systems is designed to learn through generalisation, then a hierarchically organised case-base may be required.
} 
would involve adapting a specific past case) may require a single precedent. This would be the highest ranked case retrieved from memory. Thus, refinement implies a similarity assessment phase during which the most similar past example may be isolated. For classification CBR, it is not necessary to select one specific case as no adaptation of the precedent is required. The goal of refinement in CBR is not unlike that performed by ranking systems such as SYNDROC. The difference is that SYNDROC does not have an initial selection phase, it applies its ranking formula exhaustively. El Busca and BDIS on the other hand retrieve a differential before grading the hypotheses.

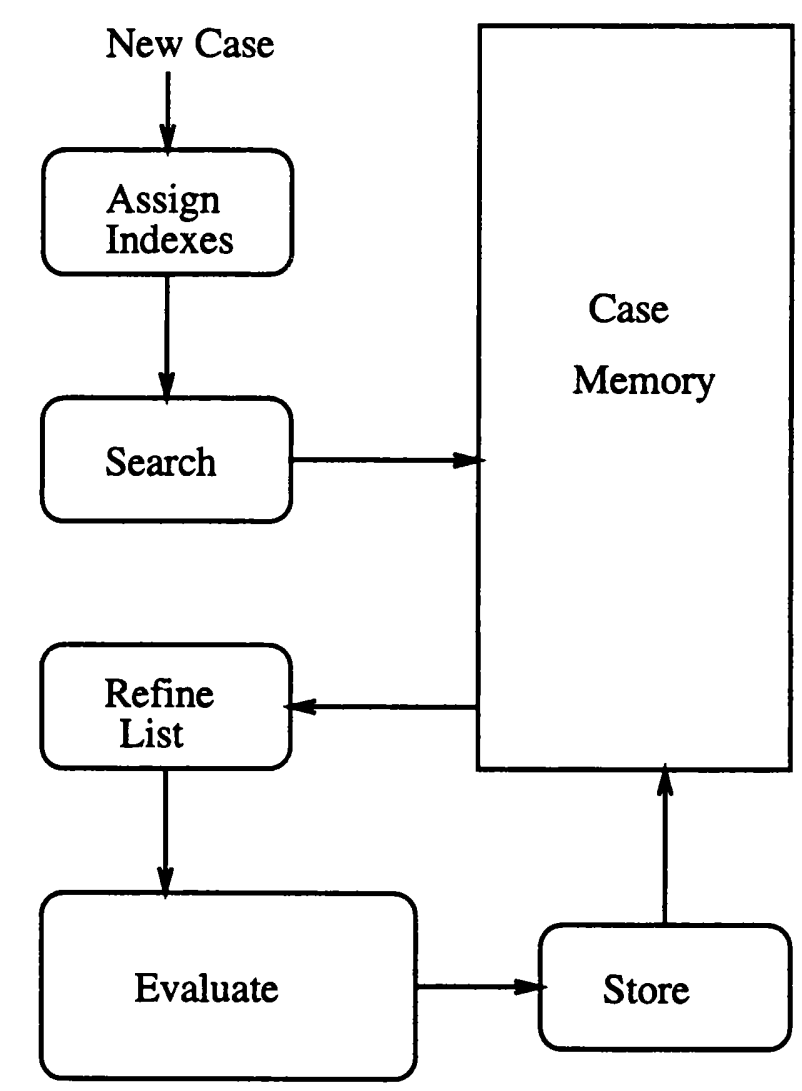

Figure 2.5: The general CBR model.

The next phase of reasoning provides a departure point for further discussion of problem-solving CBR. Following a refinement stage, a problem-solver would proceed to a number of interdependent and iterative program phases: adaptation (which would involve transforming the retrieved case to solve the current problem), test (this would check if the adapted solution works), explanation (if the proposed solution does not succeed an attempt may be made to find specific reasons for failure), repair (a second phase of 
adaptation designed to rectify problems highlighted through explanation) and storage [60]. These functional parts are enveloped by an evaluation phase in the abstracted view of CBR given in Figure 2.5. Evaluation, for the purposes of classification CBR, is essentially a decision as to whether or not one or more of the retrieved objects from case memory provides a solution. The solution, in this instance, is an indication of where in memory the new case should be stored. If the new case matches a general case (a category, or syndrome prototype) sufficiently, then the solution would be to store the case as an instance of that object (i.e., diagnosed as a specific syndrome). Alternatively the best match may be with a case previously diagnosed, which would also suggest a link to a syndrome object. If the best match is with an undiagnosed case, then the new case would be linked to the past case, but they may stand isolated within memory. In this instance, it may then be possible to abstract the common features of the two cases in order to form a new generalised case object. This procedure constitutes learning within the system, and specifically to the dysmorphology domain, may point to a new syndrome.

It has not been difficult to map aspects of dysmorphology to facets of CBR. It could be construed that a database such as LDDB is a CBR system. However, there is no automated reasoning as such. In this instance, LDDB provides the case-base whilst reasoning is performed by the physician. In LDDB, and other systems, knowledge is static. This section has highlighted the relevant aspects of the CBR model that provide focus for this work: memory organisation and similarity assessment. These topics will be discussed further in due course.

\subsection{Incremental Concept Formation}

The previous section indicated the importance of the case memory structure to a classification CBR system, specifically if it is to learn. Discussion of case-base structure and development has been deferred until this section. This is due to the fact that classification style case-based learning algorithms (CBL) have principles very much in common with another AI field, incremental concept formation. Both aim to produce an organised structure against which individual cases may be classified. For CBR systems, this structure constitutes the case memory, whilst researchers in incremental concept formation think in terms of a network of category descriptions. As with the previous section on case-based reasoning, this section highlights the key principles of such algorithms with 
reference to the dysmorphology domain.

One tenet of machine learning research is that human learning can be viewed as a gradual process of concept formation [20]. A simplistic view of this idea is that an agent (such as a physician) might observe a succession of objects or events (such as affected infants) from which a hierarchy of concepts may be induced (such as syndrome categories). The hierarchy of concepts would then summarise, and organise the agent's experience. The following high level algorithm applies:

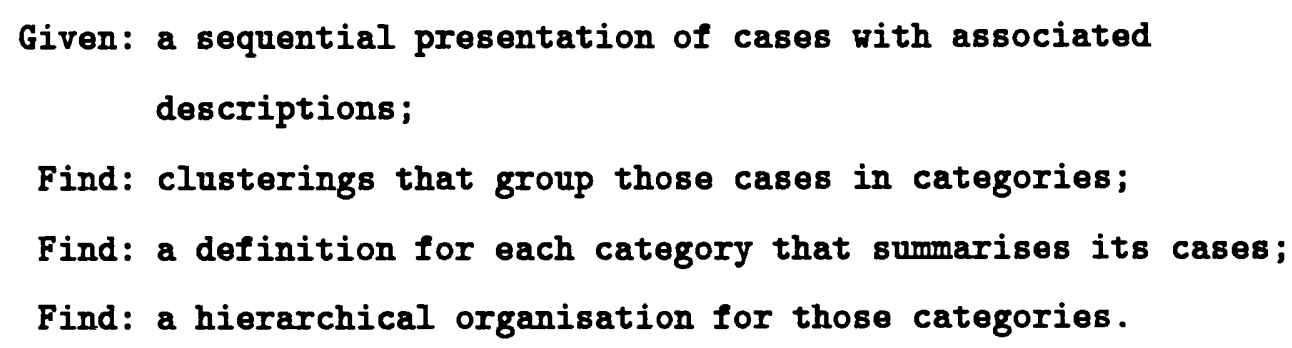

The main distinction between this type of algorithm and conceptual clustering methods $[18,52,53]$ is the constraint of incremental operation ${ }^{17}$. This constraint is seen as an important factor when viewed in the context of learning in dysmorphology which is by its very nature incremental.

The tasks of a concept formation algorithm are to help improve the understanding of the relevant domain, and subsequently enable predictions about future behaviour [20]. Understanding is enhanced by summarising case groups and generating conceptual categories. Thus, a category description infers an understanding of those cases grouped within it. Prediction is enabled by (hierarchical) category organisation and an associated search technique. When a new case enters the system, a search is initiated in order to find the best matching concept. A match may only be partial, in which case predictions may be made about missing characteristics of the new case by comparing it to the selected category. The procedures of learning in dysmorphology map well with these ideas. In dysmorphology, a prediction is effectively a diagnosis. On examination of a new patient the physician searches known syndromes (which correspond to category summaries) to find the best match. Dissimilarities between the syndrome description (which provides a list of the expected anomalies for the disorder, i.e., the predictions) and the patient may drive further examination in order to account for the hypothesis.

\footnotetext{
${ }^{17}$ Conceptual clustering covers a range of algorithmic methods including numerical techniques not unlike those employed by Preus.
} 


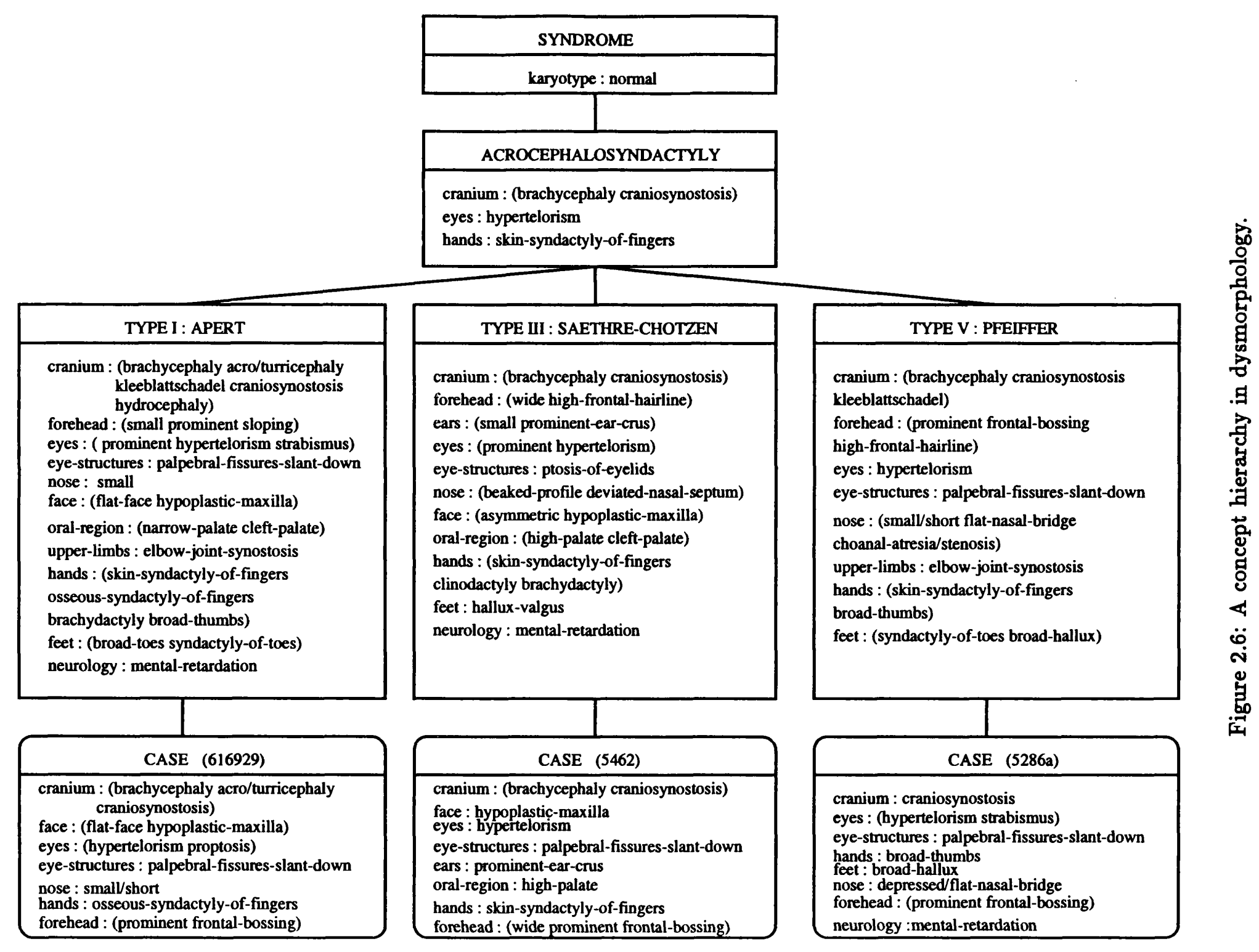


Figure 2.6 shows a concept hierarchy which is derived from the London Dysmorphology Database. This structure may be considered applicable to CBR (as a localised section of case memory) and viewed as a network of categories against which individual cases may be classified. The basic principle concerning its use is that given an incremental input of individual patients exhibiting the relevant patterns of dysmorphic syndromes a case-based learning algorithm, or incremental concept forming algorithm, would generate this type of structure. As further cases are entered the structure would evolve. That is to say, it would reorganise its structure with respect to the descriptive categories which it holds. The key aspects of such an algorithm are described below:

- An incremental concept forming algorithm creates a concept hierarchy, or a hierarchical network of concept descriptions called nodes. In dysmorphology, a concept refers to a syndrome description in terms of its pattern of dysmorphic features. A concept or node effectively forms a category against which cases or sub-categories may be classified. The node description is used to enable such classification, and is sometimes referred to as category information. There are no firm rules to determine how a concept or category information is constructed. The concept hierarchy of Figure 2.6 contains five nodes and three cases defined using an attribute-value representation.

- A concept hierarchy is ordered by generality. A concept effectively forms a category beneath which sub-categories are referenced, and above which more general parent classes are linked. The most general node of Figure 2.6 has one attribute-value pair (karyotype, normal $\vee$ abnormal) which designates whether sub-categories refer to chromosomal or non-chromosomal syndromes. The hierarchy shown consists of three non-chromosomal concepts (representing three syndromes from a known family: Apert, Saethre-Chotzen and Pfeiffer syndromes) grouped below a more general parent node (Acrocephalosyndactyly ${ }^{18}$ ). Below each of the three Acrocephalosyndactyly nodes (sub-categories are sometimes called child nodes) an instance, or specific case is stored, i.e., is classified as belonging to the relevant category (thus representing an actual patient diagnosis).

- Classification is top-down. Search through the concept hierarchy begins at the topmost general node and propagates downwards (analogous to a sorting procedure).

\footnotetext{
${ }^{13} \mathrm{~A}$ disorder characterised by a peaked head and webbed fingers and toes.
} 
Search may be complemented by a specified matching procedure in order to facilitate decisions at active nodes, i.e., an assessment would be made between the active case (an instance) and the active node (the concept at which search has arrived) in order to establish whether the case matches the node sufficiently so that search may be propagated to relevant sub-categories, or so that the case may be classified (and stored) as an instance of that category. There is some similarity here with methods for constructing decision trees [59]. However, decision trees do not store explicit descriptions on nodes as such. Also, with decision trees, the decision as to which branch of the hierarchy to search is determined by a single attribute. This constraint does not apply with incremental concept formation algorithms, which may incorporate a more general matching scheme. A further distinction is that, unlike decision tree algorithms, a case need not only be stored with respect to terminal ${ }^{19}$ nodes, i.e., individual cases may be classified, or stored, at nodes at any level in the hierarchy. This maps well onto the dysmorphology domain as cases may be linked with more general syndrome families without being diagnosed to a specific disorder.

- Concept formation algorithms are designed to operate in an unsupervised manner [20]. This is seen as a key distinction of incremental concept formation with respect to learning from example methods in which a user guides the concept formation and has control over the grouping. An important consequence is that along with deciding how to classify a new case the algorithm effectively determines the number of concept nodes.

\footnotetext{
${ }^{10}$ Terminal nodes are leaf nodes, although the concept network may be a graph structure rather than a tree.
} 


\subsection{Conclusions and Philosophy of Approach}

The major thrust of this thesis results from the mapping between aspects of the performance tasks in dysmorphology (i.e., diagnosis and research) and principles of case-based reasoning and learning. That is to say, CBR and CBL provide an intuitive architecture for a computer system dedicated to this domain. Given an organised case-base, such as that shown by Figure 2.6, diagnosis is essentially an operation of classification style casebased reasoning. It involves finding the optimum location in the case memory at which to store a new case. If this location corresponds to a generalised syndrome case (or prototype), then a (tentative) diagnosis may be inferred. If not, then storage of the new case at the best location may facilitate a memory reorganisation. Research in dysmorphology would be associated with case-based learning and the subsequent reorganisation of the case memory over time (which has similar underlying mechanics to incremental concept formation systems). Table 2.4 summarises these mappings. With these assumptions, and a desire to extend the scope of operation exhibited by previous computer systems in dysmorphology, this thesis presents a CBR architecture which is designed to incorporate such mechanisms.

\begin{tabular}{|l|l|l|}
\hline Dysmorphology & Inc. Concept Formation & Case-Based Reasoning \\
\hline $\begin{array}{l}\text { Diagnosis of a dysmorphic } \\
\text { syndrome. }\end{array}$ & $\begin{array}{l}\text { Classification of an instance } \\
\text { within the concept hierarchy. }\end{array}$ & $\begin{array}{l}\text { Location and retrieval } \\
\text { of the best matching } \\
\text { case or general case } \\
\text { in memory. }\end{array}$ \\
\hline $\begin{array}{l}\text { Establishing a new syndrome } \\
\text { by matching two or more } \\
\text { undiagnosed infants. }\end{array}$ & $\begin{array}{l}\text { Forming a new child node } \\
\text { below the root through } \\
\text { generalisation of the } \\
\text { input case with one or more } \\
\text { uncategorised instances. }\end{array}$ & $\begin{array}{l}\text { Finding that the most } \\
\text { similar precedent in } \\
\text { memory is isolated, } \\
\text { and forming a new } \\
\text { general case based on its } \\
\text { similarity with the input. }\end{array}$ \\
\hline $\begin{array}{l}\text { Establishing a revised syndrome } \\
\text { category by analysing } \\
\text { an undiagnosed infant with } \\
\text { family. }\end{array}$ & $\begin{array}{l}\text { Forming a new child node } \\
\text { below a non-terminal } \\
\text { node in the concept hierarchy } \\
\text { through generalisation of } \\
\text { the input case with one or } \\
\text { more stored instances. }\end{array}$ & $\begin{array}{l}\text { Finding that the most } \\
\text { similar precedent in } \\
\text { memory is linked to } \\
\text { a more general case, but } \\
\text { there exists sufficient } \\
\text { similarity with the } \\
\text { precedent to form a new } \\
\text { distinct prototype. }\end{array}$ \\
\hline
\end{tabular}

Table 2.4: Comparative procedures of dysmorphology, CBR and incremental concept formation.

An important consideration when establishing the thesis goals, was the real-world 
nature of the domain. Case-based reasoning research, with a reasonably stable general model, is perhaps reaching a stage at which there is movement away from the 'laboratory' towards practical applications. The dysmorphology domain promotes such a step. Whilst CBR has been described as intuitive within this medical field, the complexity, incompleteness and uncertainty of dysmorphology provide a challenge to general CBR theory. Therefore, practical considerations have, to a great extent, directed this research.

As well as recognising that complex real-world situations of any domain provide challenges to theoretical ideas from within $\mathrm{AI}$, medical fields in particular have the additional problem of scepticism from domain experts. This can only be accentuated by a field such as dysmorphology in which knowledge is uncertain. Therefore, if the aim is to propose a solution in terms of a computer model, realistic performance goals should be identified, especially with respect to learning new syndromes. An adherence to this underlying constraint has in many ways guided the Winter and Baraitser model [87]:

The recognition that several patients with a particular pattern of malformations exhibit a new syndrome is highly subjective, at least in the initial stages. It is unlikely that statistical criteria could be devised to 'prove' that a particular group of patients must have a previously unrecognised 'new' syndrome. Therefore the aim of any procedure for matching cases should be the identification of smaller subgroups of patients who share features. Once grouped, cases can be further evaluated by analysis of photographs, clinical examination, and other subjective assessments.

The experiments reported in this thesis utilise symbolic data derived from either medical records (or documentation) or computer databases. Thus, the above premise is applicable in this instance. Realistic system performance (with respect to learning new syndromes) is thus a matter of identifying possible links between previously undiagnosed cases from the data at hand. Case-based learning techniques effectively have this goal. However, groupings, or links between cases, made by a purely symbolic computer system should not be taken as proof that a new syndrome has been identified. Rather, such links provide the focus for further investigation by an expert.

One focus of this research is on the development of a case-based learning algorithm specific to dysmorphology. Chapters 4 through 6 report on a number of developmental stages with respect to a case-based learning model applied to dysmorphology. Chapter 4 
describes the development of a case-based learning algorithm taken from the literature which is utilised with experimental data specific to dysmorphic syndromes. Chapter 5 extends this program by incorporating a body of diagnostic knowledge acquired from the London Dysmorphology Database. Chapter 6 concludes the work specific to case-based learning, and analyses how a general theory of similarity assessment (taken from the psychology literature) can be introduced to further enhance the CBL model.

Whilst the learning aspect of dysmorphology and its mapping with the theoretical models of CBR and incremental concept formation provide a focus for this thesis, a background goal of the work is to develop a general CBR model dedicated to dysmorphic syndromes. In this sense, the focus on learning exists in the context of developing a default dysmorphology computer system that also addresses the diagnosis task. After all, diagnosis is the primary task when a new patient is presented to the physician. An attempt to establish a new syndrome is a secondary task, and will generally result from a failure to diagnose a case. The CBR model proposed in this thesis is one that incorporates syndromes, diagnosed cases and undiagnosed cases. It is not merely a database of unknown patients. Thus, whilst the system has an explicit focus on automated learning (unlike previous systems), it is intended also to facilitate the default task of diagnosis. The implications of this higher order goal concern the architecture of the case-base. Case-based learning and incremental concept formation rely on hierarchical memory organisation, and this is fine with respect to a localised portion of the syndrome nomenclature. However, when viewed in a broader context, the syndrome nomenclature does not readily provide a well structured hierarchy. This aspect of the domain provides a problem with regard to the more general, practical goal of the thesis. Chapter 3 addresses this problem. It describes a case-based architecture which is designed to facilitate efficient retrieval of similar entities (which could be syndrome or case records) from memory, and which provides a foundation for the learning programs described in the subsequent chapters.

Following the presentation of a dedicated CBL model, the remainder of the thesis addresses the broader view of CBR in dysmorphology. The major practical issues brought to light throughout the thesis are summarised with respect to a practical CBR model for dysmorphology. 


\subsection{Summary}

- Dysmorphology computer systems can be classified across four categories: databases, expert systems, ranking systems and visual systems. Ranking systems lie somewhere between databases and expert systems. They do not incorporate a system derived model of expert procedures, rather they utilise a standard numerical formula in order to propose a ranked differential diagnosis.

- All computer applications explicitly address diagnosis. None explicitly addresses the learning task performed by physicians.

- This work involves an explicit automation of the learning processes using techniques from artificial intelligence: case-based reasoning and learning. As well as a mapping between the learning task in dysmorphology and the mechanics of CBR that comprise learning, the diagnosis task can also be modelled by case-based reasoning.

- The mechanics of case-based learning have much in common with another machine learning field, incremental concept formation. The concept hierarchies developed by these algorithms are structurally similar to the syndrome nomenclature of dysmorphology. 


\section{Chapter 3}

\section{Case Representation and Memory Organisation}

\subsection{Introduction}

An architecture based on hierarchical categorisation can be beneficial. For the machine learning community it provides a building block for efficient, recursive algorithms. Similarly for researchers in CBR (which is a field of machine learning), hierarchical memory organisation enables a confined search strategy (i.e., search for matching entities can be selective so that not every database record is checked), and a dynamic case memory. Furthermore, in many fields hierarchical categorisation of domain entities is an intuitive representation scheme. This can be said of dysmorphology, at least to some degree. However, a problem with dysmorphology is that it is a very incomplete or weak domain. It may be that one underlying hierarchical category structure for syndromes does exist, but the current state of the field is such that this type of organisation occurs only at isolated points or with respect to confined syndrome groups. Consequently, one syndrome hierarchy would be difficult to construct (at the current time), and at the very least its structure would very broad and shallow. Such a network would not promote an efficient (confined search) retrieval mechanism for syndromes and cases.

With a hierarchical architecture like that shown by Figure 2.6 in Chapter 2, and a comprehensive set of syndromes like that stored in LDDB, the case-base would effectively have four levels: a root node, which represents the entry point to the case memory (for search to commence), a second level which stores known syndrome family prototypes, 
isolated syndromes and undiagnosed cases, a third level which stores known sibling syndromes linked to a parent prototype, and a final level which stores individual diagnosed cases (see Figure 3.1). The main feature of such a case-base is that it is very wide and shallow reflecting the mutual exclusivity of the syndrome nomenclature. Search would still be confined with the CBL algorithm, but not by very much ${ }^{1}$. The first level (for which each syndrome or case object will be matched with the new case) could potentially comprise thousands of syndrome or case objects. This is clearly an unattractive feature of the system if it is to retrieve similar objects through combined search and similarity assessment.

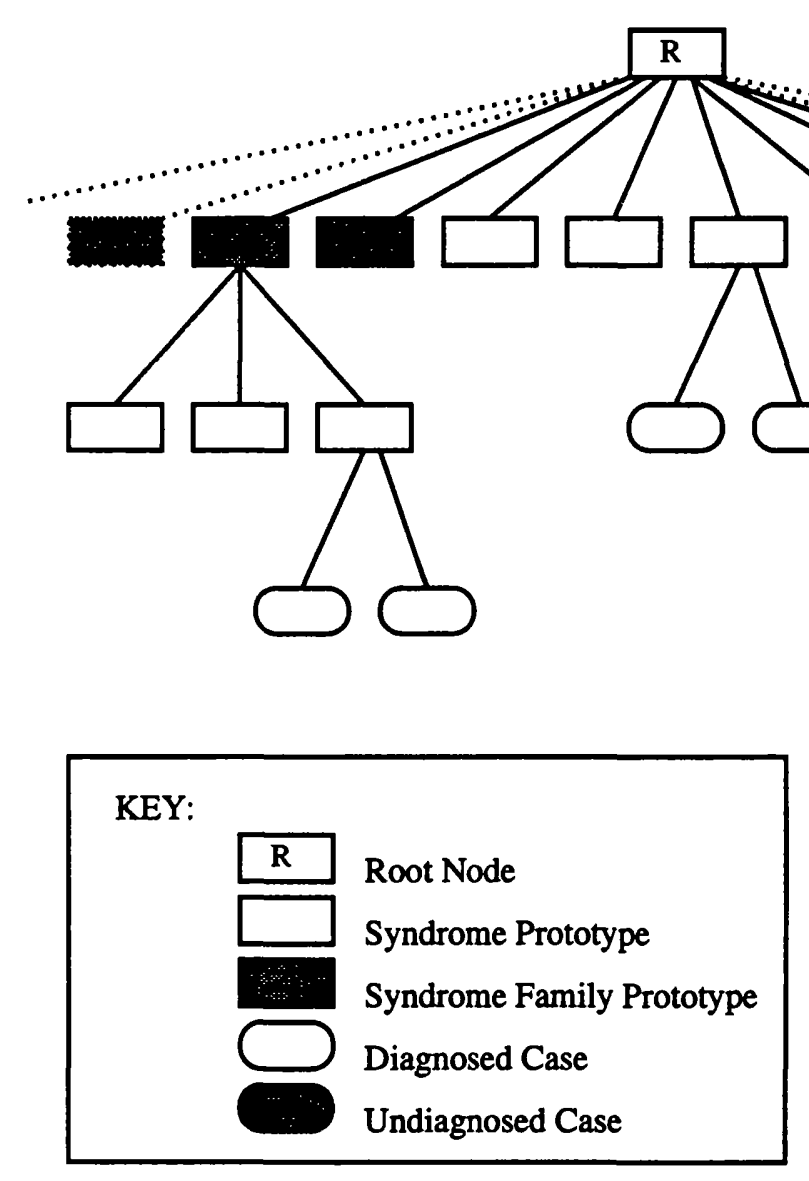

Figure 3.1: The four levels of syndrome nomenclature.

As with similarity assessment, an efficient retrieval mechanism is regarded by the CBR community as a fundamental element of a CBR system. This is one reason why hierarchical representations have been popular, they promote confined search. It is diff-

\footnotetext{
${ }^{1}$ A detailed description of how a case-based learning algorithm searches a hierarchical memory organisation is given in Chapter 4.
} 
cult to tackle this topic in isolation, however. Case representation, memory organisation and object retrieval are all important units to CBR, and are very much inter-dependent. Consequently, this chapter examines these topics together with respect to a general casebased architecture.

The motivation for the work described in this chapter is to provide a case-base architecture that can be used by a standard retrieval mechanism for diagnosis (something like LDDB), whilst at the same time providing a generic case representation and memory organisation upon which a case-based learning program can operate. A generic architecture is particularly important in this instance because the experimental data utilised originates from a number of different sources with different case representations. This is indicative of the non-uniformity of case data in dysmorphology. Thus, a generic system, in terms of allowing the relevant program functions to operate on case data from different sources with varying structure, is a valuable asset. The architecture described is, in a sense, a compromise. It is not a category hierarchy, but it does enable a confined search strategy, and it provides the underlying architecture on which the case-based learning programs described in Chapters 4, 5 and 6 operate.

\subsection{System Software}

All programs described in this thesis have been developed using Common Lisp. The software components that comprise a general CBR model (as described in this thesis) are shown in Figure 3.2. There are three basic units: a case memory which comprises a storage element and case representation, the calling procedures, i.e., the top level algorithms, and underlying utility programs which incorporate memory access routines (object retrieval and memory update functions) and matching functions. The case representation utilises the Common Lisp Object System (CLOS) and the basic storage element consists of a collection of hash tables. The utility software incorporates a separate body of program macros that have been designed to build the case memory according to the case representation. These software modules facilitate a generic system in terms of the case representation against which the calling procedures are shielded. 


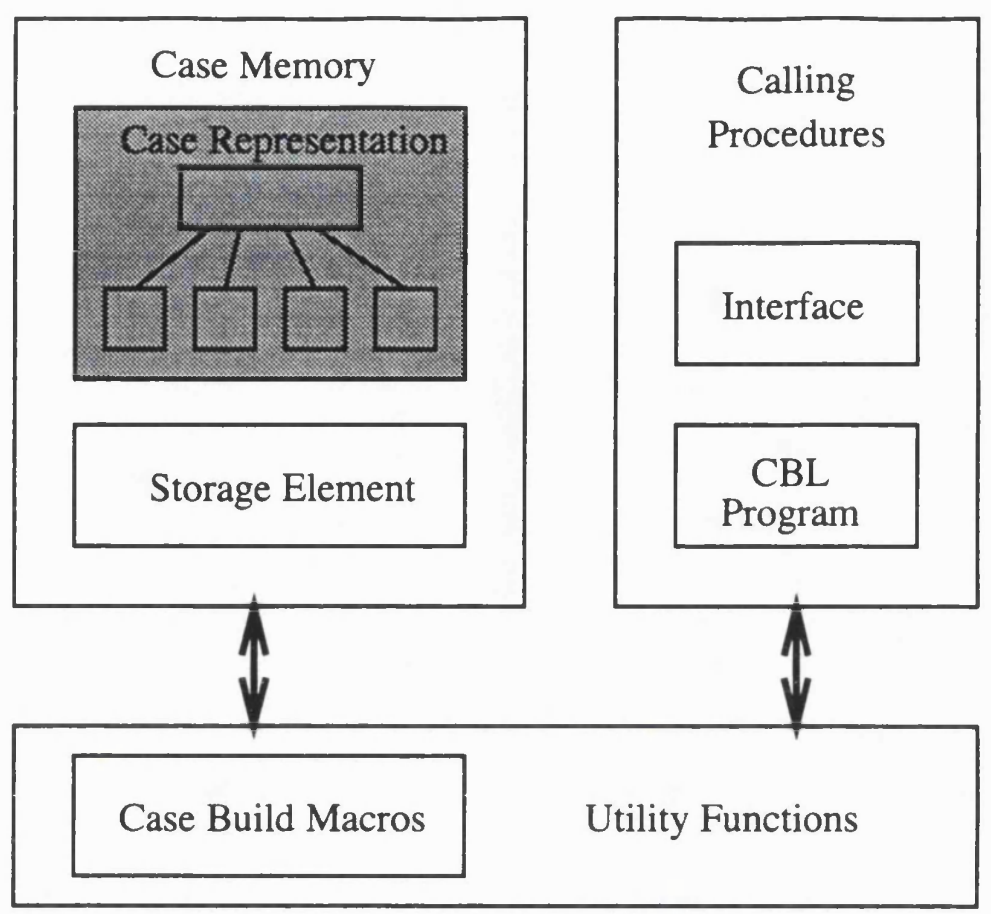

Figure 3.2: Software components of the general CBR architecture.

\subsection{Case Representation}

The Common Lisp Object System provides primitives for the straightforward development of object-oriented software $[83,71,32]$. The basic primitive is the defclass macro which enables simple declaration of object classes for use with object-oriented or framebased systems. An object class is defined in terms of a list of attributes. Every object

\section{Addendum}

A method refers to a procedure or program function that takes a specific object class as a parameter and is generic to that class. Thus, a particular class method will operate with objects of any sub-class of the aforenamed class (as its attributes will be inherited). CLOS access methods are automatically generated for each attribute of each object class that is defined. 


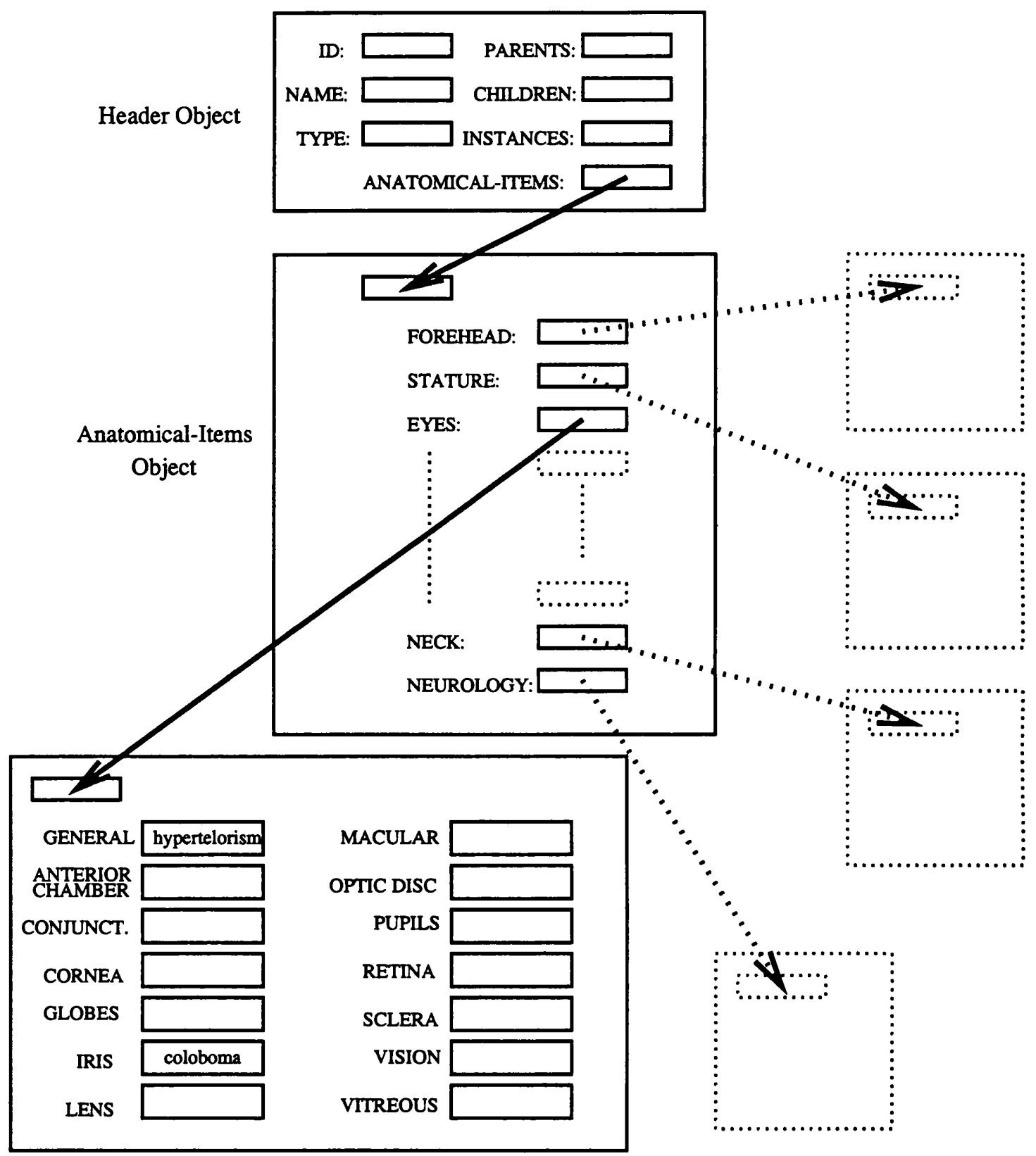

Regional Object

Figure 3.3: Object case structure. 


\subsubsection{Case Structure}

The case representation comprises a number of objects (which can also be called frames), that are linked together. Each individual case has a header, which constitutes a specific object class. On entry to the system, every case will be assigned header object instantiated by the associated defclass operator. The header object comprises a number of attributes, called slots (see Figure 3.3). One slot contains a unique identifier (which is an integer value). There is also a slot for an alphanumeric identifier, and another which states the type of record (for example, if it corresponds to a case or a syndrome). The parent, children and instances slots will contain values depending on the type of record (these are discussed in section 3.5).

The anatomical-items slot in the header object acts as a pointer to another object, the anatomical-items object. This object is the focal point of the case representation. It contains a list of slots corresponding to each anatomical component that is relevant for that particular case. Thus, for a case described by the LDDB representation, the anatomical-items object would contain a slot for each of the components listed by Table 2.1 (which shows the thirty-five clinical regions defined by the LDDB representation of syndromes and cases). Thus, it is the anatomical-items object linked to the header object which defines the representation for an individual case. Furthermore, this object points to the specific regional objects that comprise the case record. For example, the thirty-five clinical regions defined for an LDDB syndrome or case constitute thirty-five separate object classes. Each component object will contain a list of slots corresponding to each specific attribute defined for that anatomical region, which will in turn store an associated value (or list of values) corresponding to a specific abnormality. For instance, the eyes object class (as defined by LDDB) would correspond to the format shown in Table 2.2. It would contain fourteen slots corresponding to the sub-categories defined within the LDDB encoding of eye abnormalities (e.g., iris, cornea and vision). These attribute slots will contain a value if a specific anomaly exists for the case. Slots for which no abnormality is recorded will be vacant, or set to null. Along with this, a case header will only be linked (via the anatomical-items object) to regional objects for which an abnormality does exist. Thus, if a case has normal hands, there will be no corresponding object for that case, and the corresponding slot in the anatomical-items object will be null. 


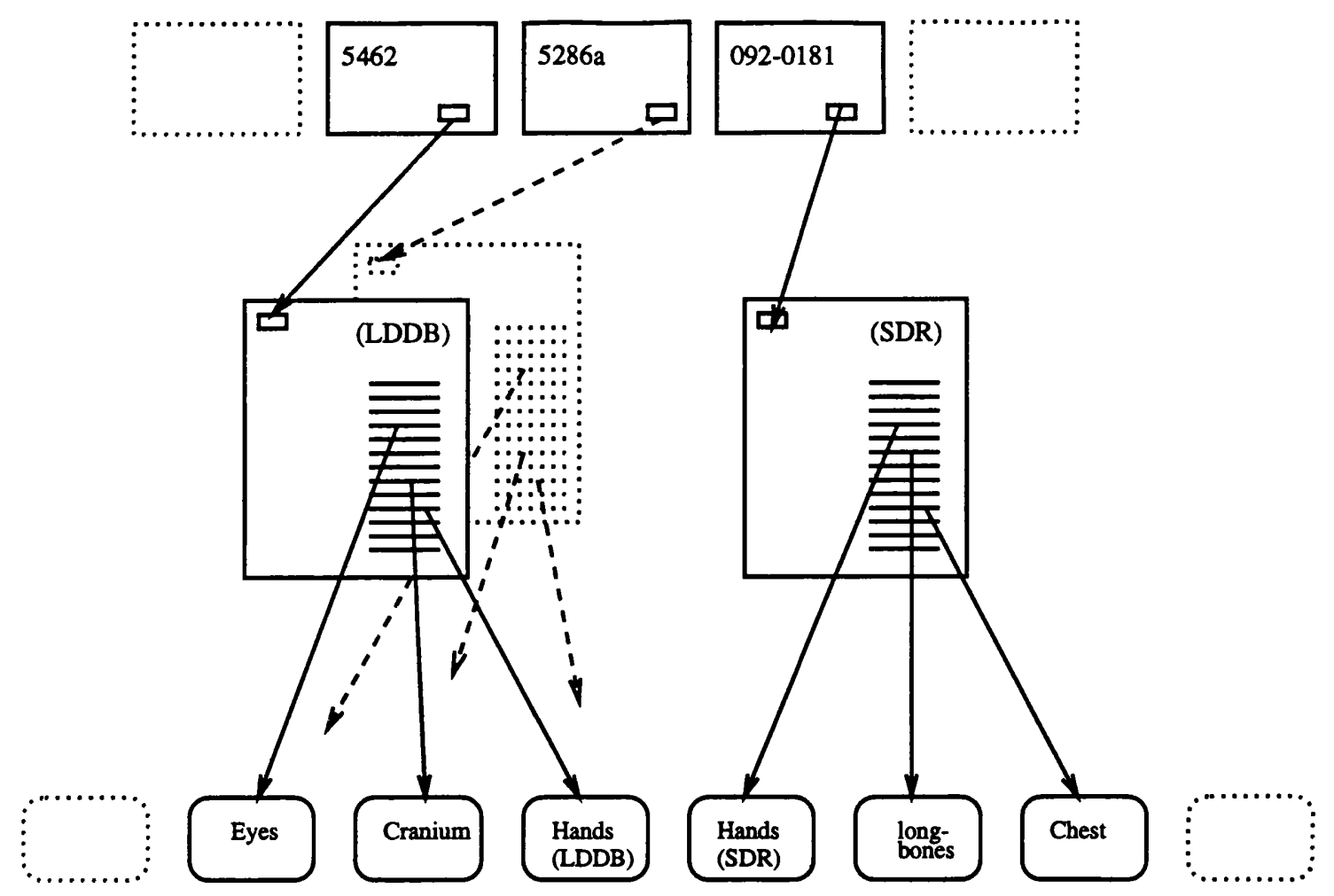

Figure 3.4: Different representations of a case co-exist.

The idea behind this structure is that different representations of a case (comprising differents set of anatomical components) can co-exist within the same case memory. Any number of clinical or radiological objects can be defined and stored within a pool of memory. The principle of this organisation is that each different case representation will have a specific anatomical-items object which only selects, or points to, the anatomical objects that are relevant for that representation. This architecture is illustrated by Figure 3.4. Furthermore, the utility programs that access case records do not require knowledge of specific object attributes, i.e., they are generic (see Section 3.3.3). Thus, any case representation can be integrated within the system provided it complies with the defining model: an anatomical-items object which defines a list of anatomical objects, and a list of attributes for each anatomical object. This condition is normally satisfied, for example, the Skeletal Dysplasias Registry (which is a source of data used in this work) comprises cases described with respect to radiological components such as spine-and-vertebrae and bone-density. There is some overlap with the thirty-five components listed by LDDB, but the SDR representation is specific to the skeleton. In the same way that each anatomical component of an LDDB case has a set of sub-categories (or attributes) and 
specific abnormalities (or values), the radiological components listed by SDR are subdivided by skeletal features and corresponding anomalies. The data from the Skeletal Dysplasia Registry is discussed in Chapter 4, and the relevant radiological components of the SDR representation are listed in Table 4.5.

\subsubsection{Case Generation and Storage}

The key to developing an environment in which alternative case representations can co-exist is in how each case object is built, or generated, and how they are accessed. Each case representation must be defined in terms of an anatomical-items class, and a class corresponding to each anatomical component. For example, the defclass construct corresponding to the anatomical-items object for the LDDB representation has thirty-five slots listed within its definition. Similarly, each of the thirty-five component object classes have slots within their definition that correspond to the relevant sub-categories (e.g., the eyes object class has fourteen slots corresponding to the iris, cornea, vision etc.,). These defclass statements are static once the initial definitions have been made. The way that actual objects are built is through the make-instance function. The make-instance function is a CLOS operator that takes an object class as a parameter. It builds an object of the specified class and initialises the object attribute values. The defclass and make-instance constructs for the LDDB representation of eye abnormalities are shown in Appendix B. A case is entered in terms of a list of dysmorphic features of the format:

\section{(item attribute value)}

where an item corresponds to the anatomical object (e.g., eyes), an attribute maps to the corresponding class object attribute (e.g., iris), and the value specifies the abnormality (e.g., coloboma), which can be a list. The listed features are passed to a macro which contains the make-instance function specific to the item class. Because the list of dysmorphic features effectively indicates which anatomical regions have an abnormality, the instantiated objects are restricted to those specified component classes. A header object and an anatomical-items object are instantiated for every case. Links between the new header, anatomical-items object, and the associated component objects for a new case are generated, resulting in a small scale object network for each case. Thus, the overall shape of a case will vary according to the abnormal regions of the body. 
For every object type that is defined by a defclass operator there exists an associated hash table with the same name ${ }^{2}$. Every object of a specified type (from all cases in the system) is stored in the associated hash table. For example, every case that has a hand abnormality will have an associated hands object which is in turn stored in the hands hash table. Thus, all objects of the same type are stored together. As component objects are only created for cases that have a related abnormality, the associated hash table will only store objects for those cases. Thus, if the system has ten cases, but only two of these have hand abnormalities, then only two hands objects would exist in the hands hash table. Each object in a hash table has a unique identifier and stores the associated case identifier that is located in the case header (this link is generated when the case is initialised).

\subsubsection{Generic Access and Utility Functions}

One of the main attractions of using CLOS for construction of the case representation and memory is that the functionality used to access cases (defined in terms of class objects) can be generic. This is desirable if the system is to integrate different case representations yet keep their respective structural idiosyncrasies hidden to the calling procedures. The key to this aspect of the system is that the underlying functionality employed to access specific abnormalities of a case has not been coded with respect to specific class objects. For example, the eyes object class has the attribute iris. Thus, to retrieve the associated value in this slot using the generic access function the call (iris eye-object) would be made, where eye-object is retrieved from the eyes hash table. This type of access routine can be readily contained within a Common Lisp macro enabling a higher level generic access function to be defined. The following Common Lisp generic utility program performs this action regardless of the class of object:

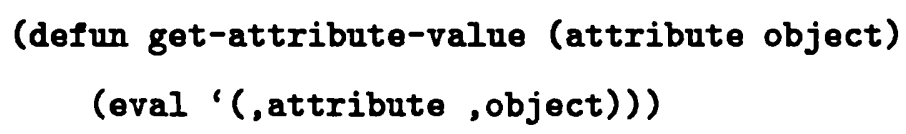

where object is any object class and attribute is any of the slots associated with that class. The text following the backquote (') defines a macro form which takes the attribute and object as parameters. The eval function then evaluates this macro form.

\footnotetext{
${ }^{2}$ Different representations may list the same component name, but with a different definition in terms of the associated attributes. To distinguish between such objects the naming convention is such that each object type must have a unique alphanumeric identifier.
} 


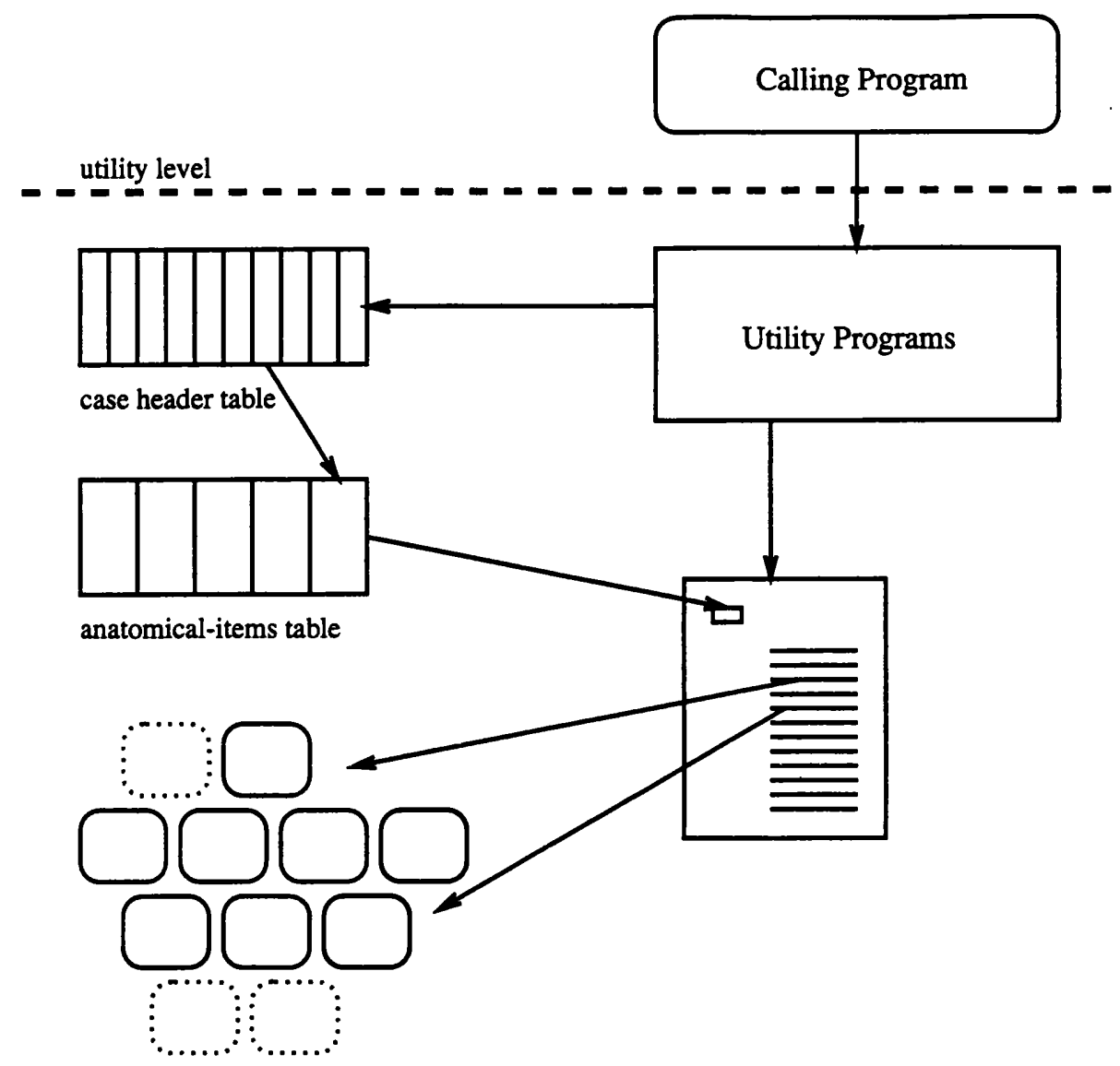

Figure 3.5: Object structure is hidden from calling procedures via the utility programs.

CLOS, and the associated Metaobject Protocol [38], provide numerous primitives to manipulate class objects. It is generally straightforward to incorporate this functionality within Lisp macros ${ }^{3}$ and create generic routines that are shielded from the representation of cases and memory. Figure 3.5 illustrates this design. When a case is under investigation, it may be necessary to list its abnormal features given its unique identifier. At this point the calling procedure, which can be either a case-based learning program or a standard retrieval algorithm, will not necessarily know which representation is the correct one for the case. The first step is to retrieve the case header object from the associated hash table. The header object will indicate the associated anatomical-items object for that case, which is retrieved accordingly. However, this could correspond to any one of many different case representations. Further processing below this point is performed by generic procedures that do not require specific details of the object structure, i.e., the

${ }^{3}$ The backquote mechanism is how macros are created in Common Lisp. 
functions that operate on the anatomical-items object, and the corresponding component objects that define abnormalities for the case, do so whatever the representation.

Technical details concerning the generic utility programs is inappropriate in the context of this thesis. However, it is enough to say that the building blocks for this aspect of the system are: the generic access functions provided by CLOS objects, a number of primitives defined by the Metaobject Protocol, and macro utilities of Common Lisp. These features allow case anomalies to be accessed by calling procedures without the need to know the specific structure of the anatomical-items object or the component objects. The consequence of this is that any representation comprising an anatomical-items definition with associated regional components can be used with the same underlying utility functions (thus, these routines do not need to be altered when a new representation is introduced).

\subsection{Memory Organisation and Indexing}

A crucial feature of the memory organisation is that all objects of the same class are stored together in the same hash table. Thus, all header objects are stored in one hash table, which only stores header objects. Similarly all anatomical-items objects (for a given representation) are stored together, as are all (LDDB) eyes objects. Every object, whatever the class, has a unique identifier. Furthermore, the anatomical-items object and all the associated component objects for a given case, store the unique case identifier as well. These identifiers are used to form the links between the different objects that form a particular case and are generated when a case is created. An individual case, therefore, does not exist within memory as a complete unit. It is distributed throughout different locations in memory. Figure 3.6 illustrates this linkage.

Another important aspect of the case memory is that syndromes are structured and stored in exactly the same manner as cases. The only distinction between a case and a syndrome is that the object-type slot in the header object is set to the symbol CASE or SYNDROME respectively. Thus, the hash tables that hold objects of a specific class can store both case and syndrome objects.

This distributed storage of cases and syndromes in memory promotes a confined search strategy without the requirement of a hierarchical organisation. Rather than commencing search at the top-level node in a concept hierarchy, with the distributed 


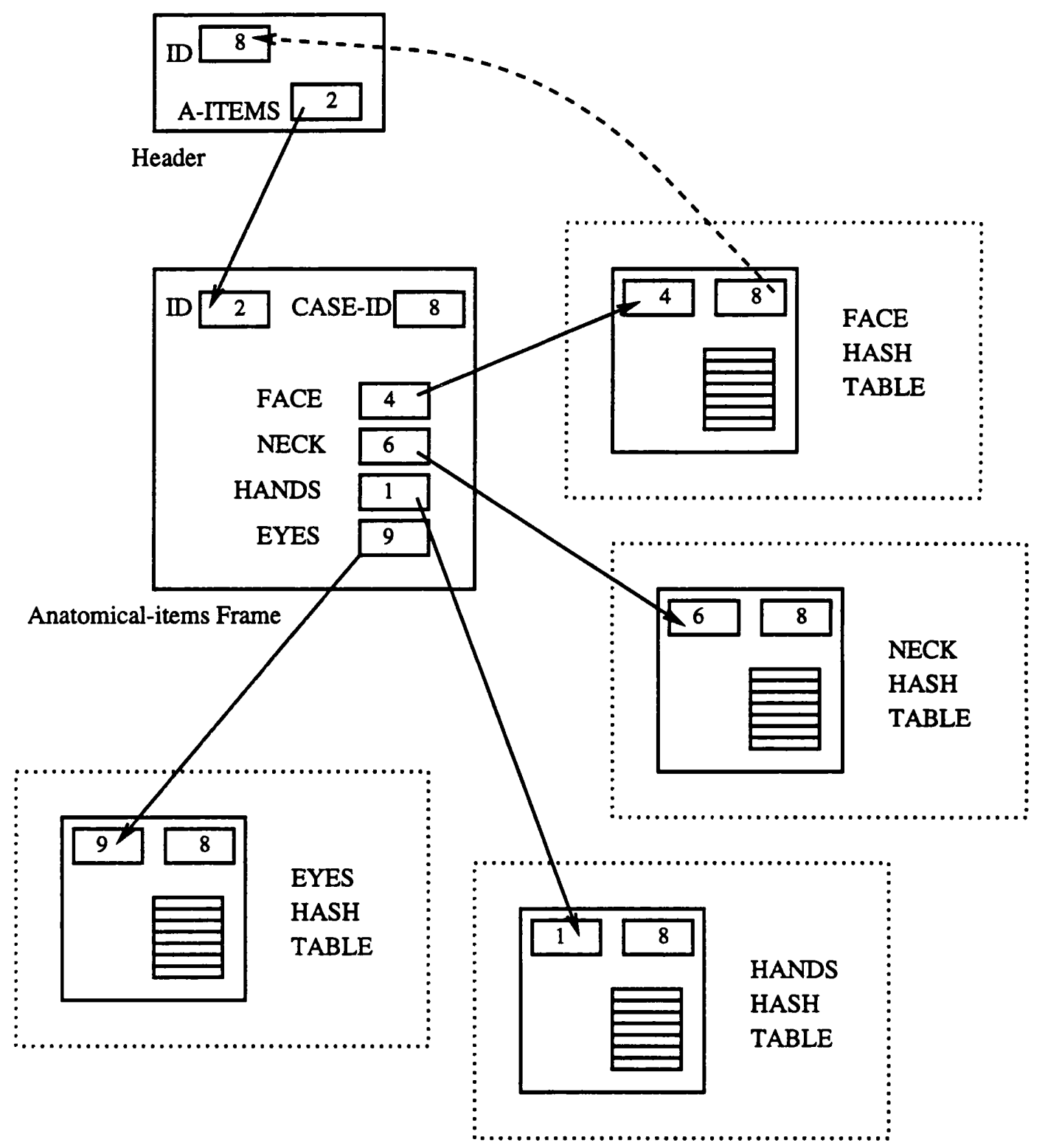

Figure 3.6: Distributed storage of a case. 
case-base organisation search may commence at a localised storage point, i.e., a specified hash table. For example, suppose a user wants to retrieve all syndromes that list the abnormality (eyes iris coloboma). The associated retrieval algorithm can immediately confine its search to the place in memory where the eyes objects are stored. It then only needs to examine the objects in that particular hash table (using the generic access function for the iris slot). The identifiers of matching header objects are simply returned to the calling procedure, which can then retrieve the relevant header objects and examine the object-type slot in order to select the corresponding syndromes. Thus, only two points in memory are accessed in this instance. The eyes hash table and the headers hash table. The first step involves accessing every object in the component table. The final step involves retrieving the header objects specified by the list of case identifiers returned by the first hashing procedure.

\subsubsection{Indexing in Distributed Case Memory: An Extended Example}

This section gives an example which demonstrates the retrieval mechanism that is possible with the distributed CBR architecture. For this example, syndrome records from the LDDB system are used. The objective here is to illustrate how this case-based structure can enable the default task of object retrieval (which maps onto the diagnosis task). Furthermore, whereas search with a flat database structure such as LDDB would involve checking each syndrome record, search within the distributed case-based organisation is confined.

In order to proceed, however, it is necessary to elaborate on how symbolic matching is performed in the CBR system, and how this compares with LDDB's equivalent process. In LDDB, individual dysmorphic features are compared in terms of their three-level numeric codes, which enables loose coupling (a matching procedure described in Chapter 2). This work has used the triplet notation (item attribute value) to represent individual abnormalities, where item, attribute and value are Lisp symbols [71]. Common Lisp provides a number of predicates designed specifically to evaluate whether or not two or more symbols match. These predicates are employed by the relevant utility functions for the purpose of matching dysmorphic features. Furthermore, a utility function performs an equivalent operation to the loose matching of LDDB. The retrieval algorithm employs this utility function, which in turn allows the symbolic triplet (item attribute abnormal) to match any feature described by item and attribute. In other words, it allows the same 


\begin{tabular}{|l|l|l|l|l|r|}
\hline build & 179 & teeth & 335 & lower limbs & 408 \\
\hline stature & 620 & voice & 106 & feet & 519 \\
\hline cranium & 775 & neck & 164 & blood vessels & 57 \\
\hline hair & 342 & back \& spine & 466 & endocrine & 137 \\
\hline forehead & 266 & thorax & 686 & haematology & 153 \\
\hline ears & 665 & abdomen & 426 & muscles & 124 \\
\hline eyes & 880 & pelvis & 124 & joints & 248 \\
\hline eye structures & 514 & genitalia & 362 & neurology & 803 \\
\hline nose & 466 & urinary system & 317 & skeletal & 408 \\
\hline face & 644 & upper limbs & 350 & skin & 547 \\
\hline mouth & 353 & hands & 844 & gestation/delivery & 0 \\
\hline oral region & 505 & nails & 205 & & \\
\hline
\end{tabular}

Table 3.1: Number of stored objects per clinical region for 1885 LDDB syndromes.

type of general matching as LDDB, albeit with symbols instead of numeric codes.

As well as the individual cases (which are used in experiments with the case-based learning programs described in the following chapters), 1885 syndrome records have been made available from LDDB. The format of LDDB syndromes is the same as for cases, and each syndrome is denoted by a list of three-level numeric codes corresponding to the feature nomenclature described by Table 2.1. In order to utilise this representation, therefore, thirty-seven different object classes are defined by the defclass construct: a header class, an anatomical-items class, and a class corresponding to each of the thirtyfive clinical regions denoted in Table 2.1. Table 3.1 shows the distribution of objects for each of the component classes when the $\mathbf{1 8 8 5}$ syndrome records are entered into the casebase. For instance, of the 1885 syndromes, 880 list at least one eyes abnormality. Thus, 880 eyes class objects are created. Accordingly, there are 1885 header and anatomicalitems objects, and the corresponding (integer) identifiers run from 1 to 1885 .

Figure 3.7 shows a suite of retrieval functions that constitute the retrieval program. The functions Retrieve and Select employ a list notation for an index, which comprises those features against which syndrome or case entities are matched. Each item in the list is a symbolic triplet of the form (item attribute value). The functions Retrieve-Objects and Select-Objects take one specific abnormality as a parameter in order to retrieve those objects of the class item that exhibit the specific anomaly denoted 
Input: A list of feature triplets I.

Output: A list of matching case identifiers from memory.

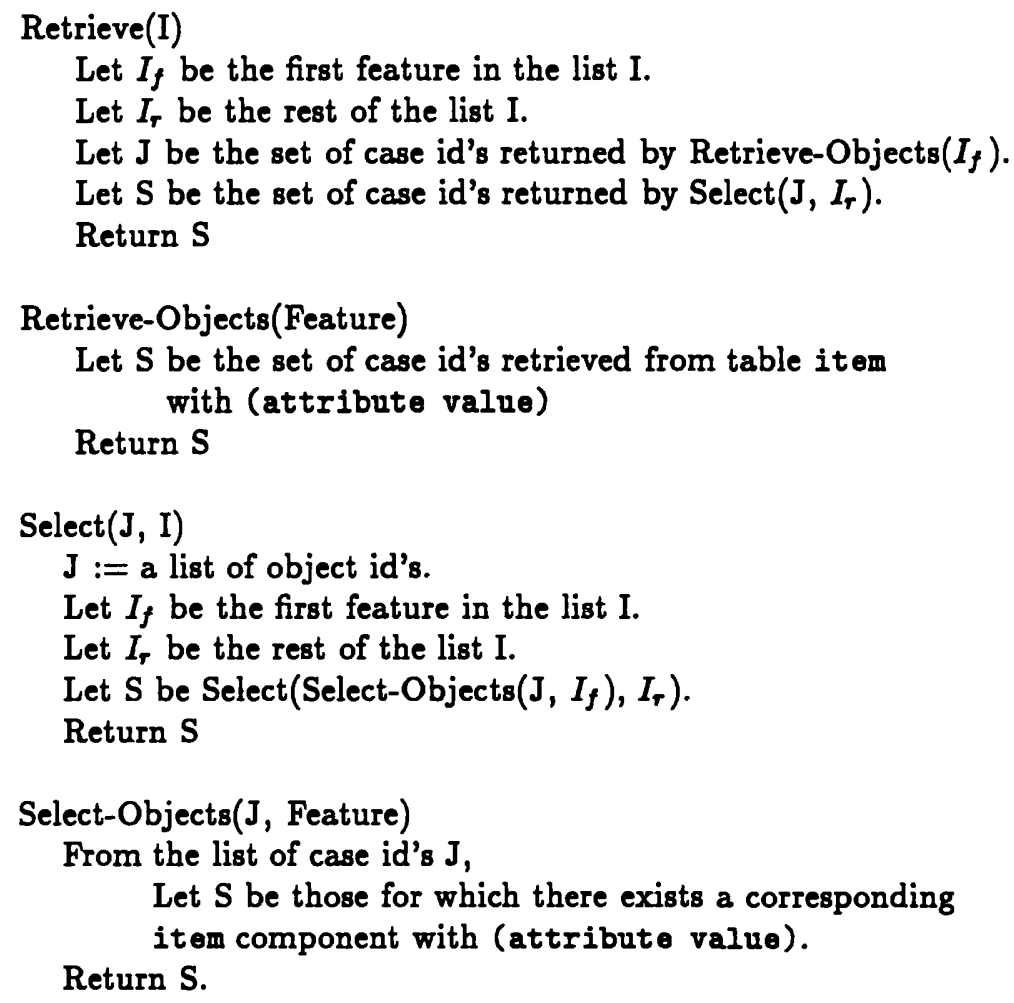

Figure 3.7: Object retrieval functions.

by the attribute and value (note that value could be the general form abnormal). The latter of these two functions is passed a specific list of case identifiers so that it can check (or select) only these from the relevant hash table. The Select function is similar, but makes recursive calls in order to process the rest of the index (those features that comprise the index without the first listed triplet).

Chapter 2 described the mode of operation of LDDB using an example index of 07.06.03, 15.01 and 32.01. The corresponding symbolic triplets produce the index:

\section{((eyes iris coloboma) (neck general abnormal) \\ (neurology general abnormal))}

As expected, when this index is used with the case-based memory, the same five syndromes are retrieved as those listed in Table 2.3. Figure 3.8 illustrates this retrieval process. The Retrieve function selects the first feature of the index, (eyes iris 


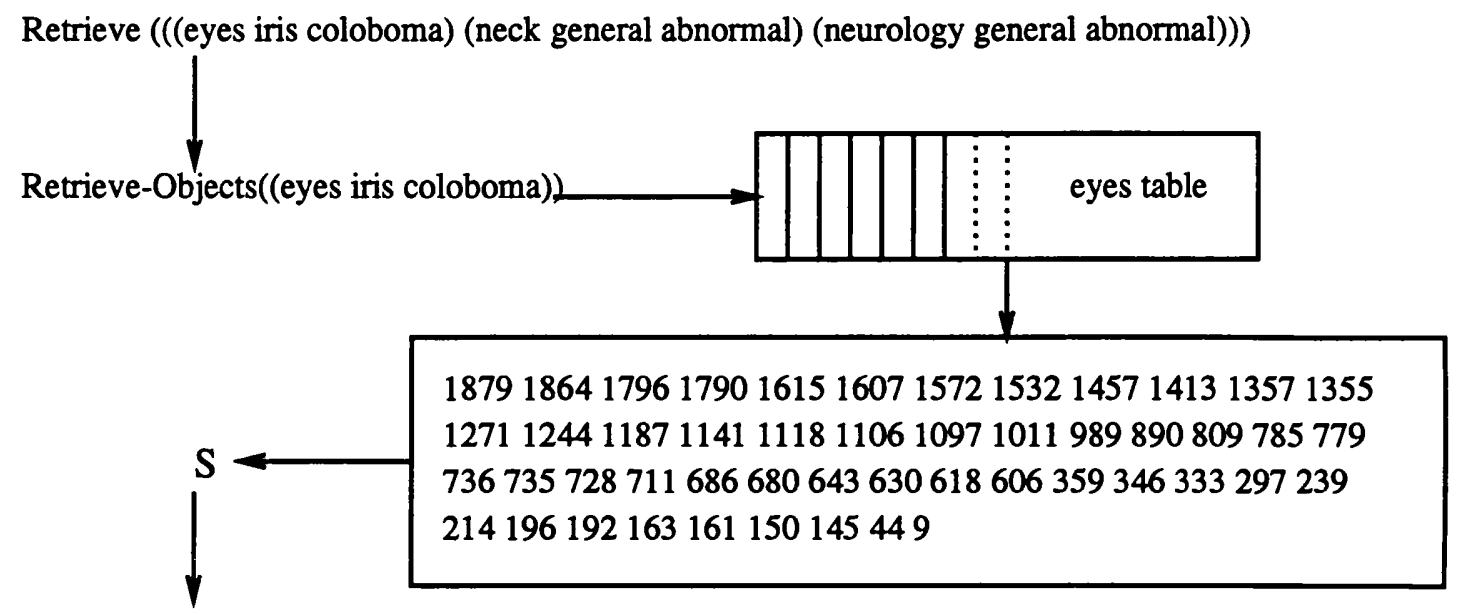

Select(S, ((neck general abnormal) (neurology general abnormal)))

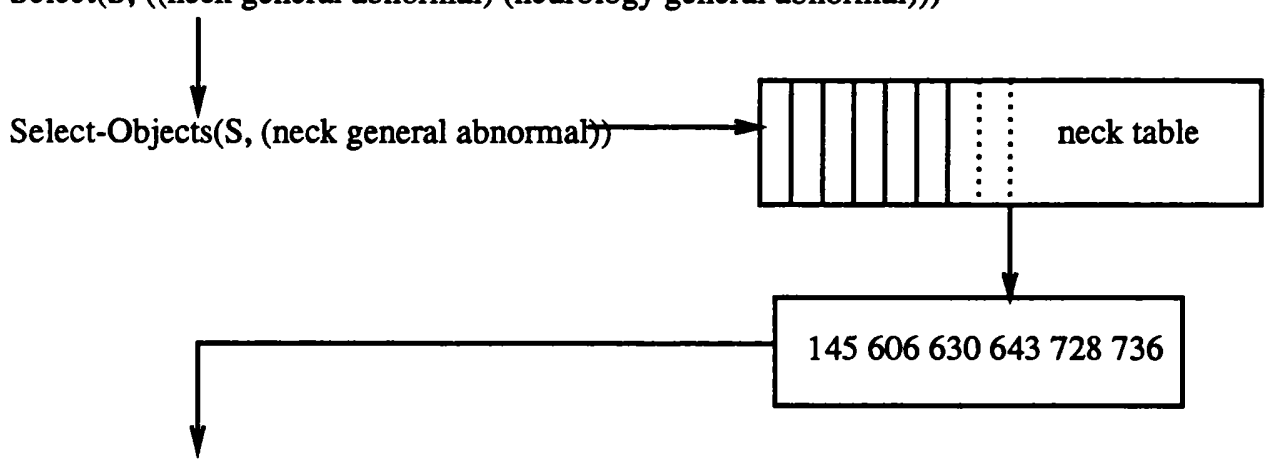

Select-Objects((145 606630643728 736), (neurology general abnormal))

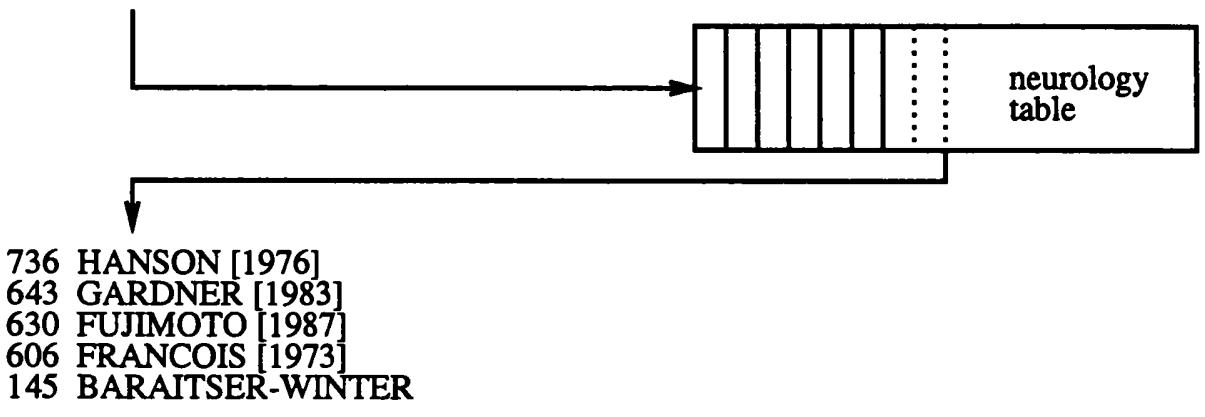

Figure 3.8: Retrieval of syndromes with (eyes iris coloboma), (neck general abnormal) and (neurology general abnormal). 
coloboma), and passes this to the Retrieve-Objects function, which matches the specified feature with each object in the eyes hash table (using the iris access function). In total, 45 of the 880 eyes objects have this anomaly listed within the iris attribute. The syndrome identifiers within the relevant slots of these 45 objects are then passed to the Select function along with the remaining parts to the index: (neck general abnormal) and (neurology general abnormal). This function then makes a call to Select-Objects, passing the list of 45 syndrome identifiers and the first item of the remainder of the index, (neck general abnormal), as parameters. The Select-Objects function checks the neck hash table with respect to the $\mathbf{4 5}$ listed syndromes, and returns 6 identifiers corresponding to those syndromes that have a general neck abnormality. This list of 6 syndrome identifiers is then passed as a parameter, along with remaining index item (neurology general abnormality), in a recursive call to the Select function. This results in a search of the neurology hash table with respect to the 6 listed syndromes. Five of these have a general neurology abnormality, and thus, match the full index. This final group correspond to those syndromes listed in Table 2.3 and is shown in Figure 3.8.

\subsubsection{Discussion}

Unlike a flat database such as LDDB, the distributed case-based architecture and the retrieval functions shown in Figure 3.8 promote a confined search for matching entities. In the above example $931(880+45+6)$ matches are performed. With a flat database comprising the 1885 syndrome records of LDDB, search would comprise 1885 comparisons. Furthermore, every retrieval procedure with a flat syndrome database would involve comparing the index against each syndrome record irrespective of how large (or small) the index is. With the distributed organisation described in this chapter, the search procedure will vary according to the size of the index and the relevant storage elements that correspond to anatomical components.

A distributed approach to database design is certainly not new. It would not be too difficult to design an equivalent relational model and utilise an associated query language to retrieve case or syndrome records ${ }^{4}$. However, standard database packages do not offer the sort of functionality that has been used to develop the generic aspect of the case-

\footnotetext{
${ }^{4}$ It is interesting to note that LDDB is written in a relational database language, but is not relational in design.
} 
based system. In a relational database system, a new case representation, resulting in a new relational design, would also necessitate new query programs. Also, the case-based architecture has the additional goal of allowing a CBL algorithm to operate upon it, and subsequently facilitate memory reorganisation.

\subsection{Case-Based Learning with Distributed Memory}

The previous sections have described two important aspects of the case-based architecture:

- The case-base is a distributed organisation in which object retrieval, and hence a diagnostic search procedure, is confined without the requirement of a category hierarchy.

- The underlying functionality of the system is generic with respect to the case representation. Thus, the calling procedures can perform their tasks without knowledge of the specific case representation.

Section 3.4.1 gave an example of the (differential) diagnostic procedure in which the calling procedure was an object retrieval program, which in turn retrieved syndrome identifiers. The program knew which case-base entities were syndromes rather than cases by checking the object-type slot in the header object of each record. Chapters 4 , 5 and 6 describe experiments in which the calling procedure is a case-based learning program. As with the object retrieval program, the CBL program operates 'on-top' of the underlying generic case-based architecture.

It has been noted that case-based learning (and incremental concept formation) procedures rely on a hierarchical category structure. Whilst the case-based organisation described in this chapter is distributed rather than hierarchical, a hierarchical network can be constructed within this architecture. This takes the form of links between the parent, children and instances slots of header objects. That is to say, one case-base entity can be linked (effectively) below another (more general) case-base record by creating a link between the respective parent and children slots of the header objects. In this way, an individual case having a particular diagnosis can be linked to the respective syndrome record via its header object. In this instance, the case would have a link from the parent slot of its header object to the children slot of the relevant syndrome header object. 
The calling procedure would know which entities are cases and which are syndromes by checking the object-type slot of each header. This set-up is specific to syndromes and diagnosed cases, however. In terms of a more general concept hierarchy (which is what is developed by the case-based learning programs described in the following chapters, and such as that shown in Figure 3.1), parent and children entities are not necessarily syndromes or cases. They could pertain to general categories such as syndrome families, or undiagnosed cases. In the former example, the parent of a syndrome record would be a general syndrome family, and in the latter example, the parent of an undiagnosed case would be the root. To enable a general concept hierarchy to be constructed, and in order to allow a case-based learning program to generate a category network with the distributed architecture, two different values for the object-type attribute of a header object are defined: NODE and ROOT. The case-based learning programs described in the following chapters do not operate in terms of syndromes as such, they work with a root, individual cases, and generalised cases called nodes. Definitions for these entities are given in Chapter 4. In this section, the objective is to demonstrate how the underlying software (which is hidden from the case-based learning program) can operate in terms of a hierarchy without physically storing objects as hierarchical units.

Figure 3.9 illustrates how a concept hierarchy is derived through links between header objects. Whilst storage is non-hierarchical, a concept hierarchy is effectively created by generating links between header objects (N.B. linkage only involves header objects). An individual case cannot have any children or instances linked below it. A generalised case, or node, can have both sub-nodes (listed in its children slot) and individual cases (listed in its instances slot) linked beneath it. Unclassified cases are linked to the instances slot of the root. The root node will only comprise a header object. It is effectively a null entity which is only defined for use with a case-based learning program (and the associated concept hierarchy). It is important to note that an individual case can be linked to more than one node (e.g., Case 12, which is linked to both Node 2 and Node 3 ). The concept networks generated by the CBL programs are designed to model the actual category structure that exists in dysmorphology, and so it is important that this aspect of category linkage is provided for. 
Header Hash Table

\begin{tabular}{|c|c|c|}
\hline \multirow{4}{*}{$\begin{array}{l}\quad \mathrm { ID } \longdiv { 1 } \\
\text { parents } \\
\text { children } \\
\text { instances }\end{array}$} & TYPE & \multirow[t]{2}{*}{ ROOT } \\
\hline & & \\
\hline & 2 & \\
\hline & 89 & \\
\hline \multirow{4}{*}{$\begin{array}{l}\quad \mathrm{ID} \lcm{2} \\
\text { parents } \\
\text { children } \\
\text { instances }\end{array}$} & TYPE & NODE \\
\hline & 1 & \\
\hline & & \\
\hline & 12 & \\
\hline \multirow{4}{*}{$\begin{array}{l}\quad \mathrm{ID} \lcm{\square} \\
\text { parents } \\
\text { children } \\
\text { instances }\end{array}$} & TYPE & NODE \\
\hline & 1 & \\
\hline & 4 & \\
\hline & 12 & \\
\hline \multirow{4}{*}{$\begin{array}{l}\quad \mathrm{ID} \lcm{\square} \\
\text { parents } \\
\text { children } \\
\text { instances }\end{array}$} & TYPE & NODE \\
\hline & 3 & \\
\hline & 7 & \\
\hline & 6 & \\
\hline \multirow{3}{*}{$\begin{array}{l}\qquad \mathrm{ID} \lcm{5} \\
\text { parents } \\
\text { children } \\
\text { instances }\end{array}$} & TYPE [ & CASE \\
\hline & $\overline{4}$ & \\
\hline & & \\
\hline \multirow{3}{*}{$\begin{array}{l}\quad \mathrm{ID} \lcm{\square} \\
\text { parents } \\
\text { children } \\
\text { instances }\end{array}$} & TYPE [ & CASE \\
\hline & 4 & \\
\hline & & \\
\hline \multirow{4}{*}{$\begin{array}{l}\quad \mathrm{ID} \lcm{\square} \\
\text { parents } \\
\text { children } \\
\text { instances }\end{array}$} & TYPE [ & NODE \\
\hline & 4 & \\
\hline & & \\
\hline & $10 \quad 11$ & \\
\hline
\end{tabular}

Header Object Linkage

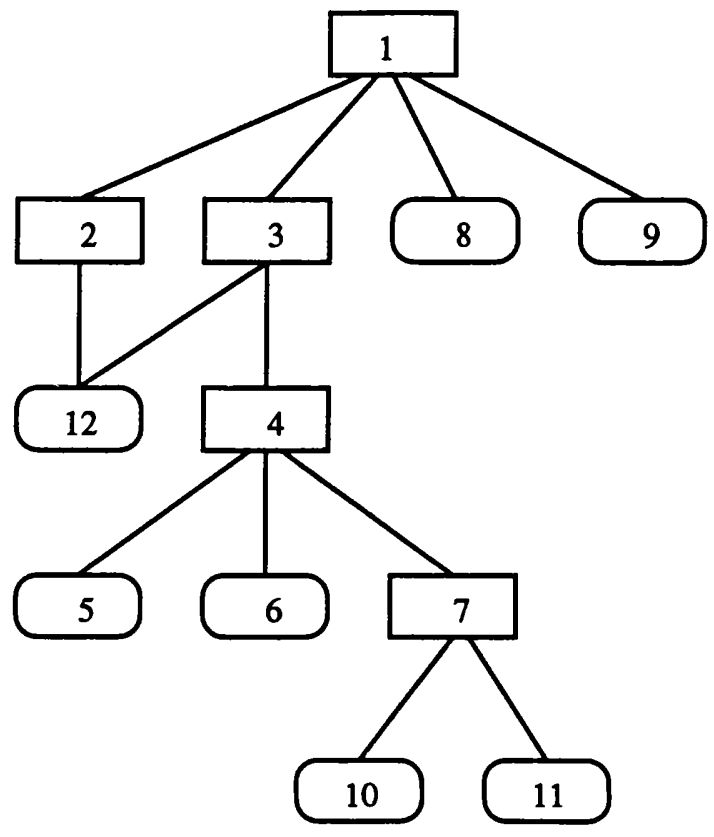

KEY:

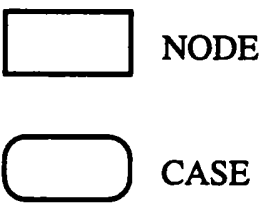

Figure 3.9: Hierarchical linkage of header objects. 


\subsection{Conclusions}

This chapter gives some technical detail of how case memory is constructed. The design involves a distribution of stored objects across the case memory such that retrieval of matching entities is facilitated by confined search. Thus, the system can achieve the default performance task of a dysmorphology database by retrieving similar database

\section{Addendum}

It should be noted that the generic design of the case representation and memory described in Chapter 3 involved considerable novelty of software design and development. Furthermore, it should be emphasised that this phase of the work provides an innovative focus of research and ultimately a significant contribution to the original research content of the thesis. The research contribution of the CBR architecture is further addressed in Chapter 8.

'I'he following chapter describes a case-based learning algorithm m detall, and reports on experiments with the CBL program which utilise real data from the dysmorphology domain (and which originates from different sources with associated differences in their case representations).

\subsection{Summary}

- A broad concept hierarchy spanning all dysmorphic syndromes is generally not accepted, and by its very nature (shallow and wide) it would not promote search that was significantly more efficient than a flat database. Efficient retrieval of casebase entities, however, is viewed with some importance by researchers in CBR. The distributed case-based architecture that is described allows search to be confined without hierarchical constraints.

- The case-base has been developed using Common Lisp and features of the Common Lisp Object System (CLOS). This software has been useful in developing generic utility programs that enable the memory organisation to be generic with respect to case representation. 
- Both syndromes and cases are constructed and stored in exactly the same way, using the same storage elements. The underlying utility functions can therefore operate on all objects irrespective of whether they belong to a syndrome or case.

- The calling procedures are hidden from specific organisational details. If the calling procedure is a case-based learning program, there is an additional requirement of enabling the generation of a concept hierarchy. This is achieved through links between the parent, children and instances slots of the header object for each case-base entity.

\section{Addendum}

It should be emphasised that although the data structures used for syndromes and cases are equivalent for use with the model of case-based memory these entities are distinct. A case refers to an actual patient, whereas a syndrome refers to the description of a recognised disorder. 


\section{Chapter 4}

\section{Experiments with a Case-Based Learning Algorithm}

\subsection{Introduction}

The goal of the work described in this thesis is to develop a case-based system that, whilst providing the kind of facility offered by a database such as LDDB (which helps establish a manageable differential) or SYNDROC (which also ranks the differential), offers explicit mechanisms for learning. In order to achieve this, the CBR system must comprise prototypical syndrome entities and a search mechanism for retrieving them. If the system is to learn, then it must also store individual cases and employ a learning algorithm to manipulate them. Case-based learning algorithms can effectively perform both performance tasks. That is to say, CBL enables the incremental concept formation approach to learning, but also provides an algorithm for retrieval of similar entities. $\mathrm{CBL}$, in this sense, provides a generic term for both incremental concept formation and classification style case-based reasoning.

The UNIMEM program developed by Lebowitz $[49,50]$ may be categorised as generic CBL and for the purposes of this research provides an algorithm with which to commence experimentation ${ }^{1}$. The UNIMEM algorithm has dynamics with much similarity to Kolodner's early case-based memory system CYRUS $[42,43]$, and has also been compared to incremental clustering systems such as Fisher's COBWEB [20]. There are other

\footnotetext{
${ }^{1}$ Lebowits has described the concept hierarchies formed by UNIMEM as 'generalisation-based memory'.
} 
important reasons why the selection of UNIMEM is a good choice. UNIMEM places cases into more than one category if sufficient similarity dictates. Thus, categories overlap and do not form disjoint partitions (a clustering approach sometimes referred to as clumping [18]). Also, when a case is compared to a conceptual category in UNTMEM, it is not required to match the concept on all listed features. That is to say, the overlap between the case and the concept does not have to be a conjunction of all the concept features in order for the match to succeed (and for the case to be classified accordingly). This type of comparison is sometimes referred to as polymorphy or polythetic matching $[28,29,18]$. Both clumping and polymorphy are characteristics seen in dysmorphology. When a firm diagnosis cannot be made, a patient can be linked to more than one syndrome. Furthermore, it has already been noted in Chapter 1 that a firm match between an infant and a syndrome is usually based on a conclusive sub-set of principal anomalies. A further attraction of UNIMEM is that its matching operators are fairly independent from the searching operation of the algorithm. With Fisher's COBWEB [20], and later variations such as CLASSIT and EXWEB [62] (which are all incremental concept formation algorithms that have utilised medical test data), program operation is very much tied to the similarity metric. The searching performed by UNIMEM does, of course, work in tandem with its similarity metric, but its matching functionality is modular, allowing the incorporation of alternative similarity metrics within the basic algorithm.

\subsection{Discrimination Networks}

UNIMEM is a discrimination network forming algorithm. A discrimination network is a concept hierarchy which consists of a set of nodes ordered by generality. Each node in a discrimination network represents either an instance (i.e., an actual case), or a concept (i.e., a class of instances or sub-classes). Terminal nodes are always instances, and nonterminal nodes are concepts. Each node has an associated description which will vary in structure according to the implementation. The UNIMEM program constructs nodes in terms of a list of features denoted by attribute-value pairs. The description associated with a non-terminal node defines a concept. The children of a node are either subnodes (sub-categories) or instances. Children are located below a parent node because they share the features listed in the parent node description. A concept hierarchy is a discrimination network when a child node is described only by the features that do not 
overlap with the parent, i.e., the features that discriminate it from the parent. Thus, when an individual case (or instance) is integrated within a discrimination network it is stored (as a terminal node) beneath a node with which it has matched (i.e., overlapped with the parent node's description), and is constructed in terms of the features that do not match the parent node. Furthermore, the parent may itself be a sub-class which has also been constructed by its discriminating features. In this sense, an instance is classified by its location in the discrimination network. That is to say, it is a member of the category defined by the parent node concept. The features that then constitute an individual case, or sub-class, are therefore the features that differ from the category under which it is classified. In this sense, the features of an instance, or sub-class, index the relevant child node, i.e, they point to its distinctive features. Figure 4.1 illustrates this structure and shows how five instances, described along four attributes (colour, shape,

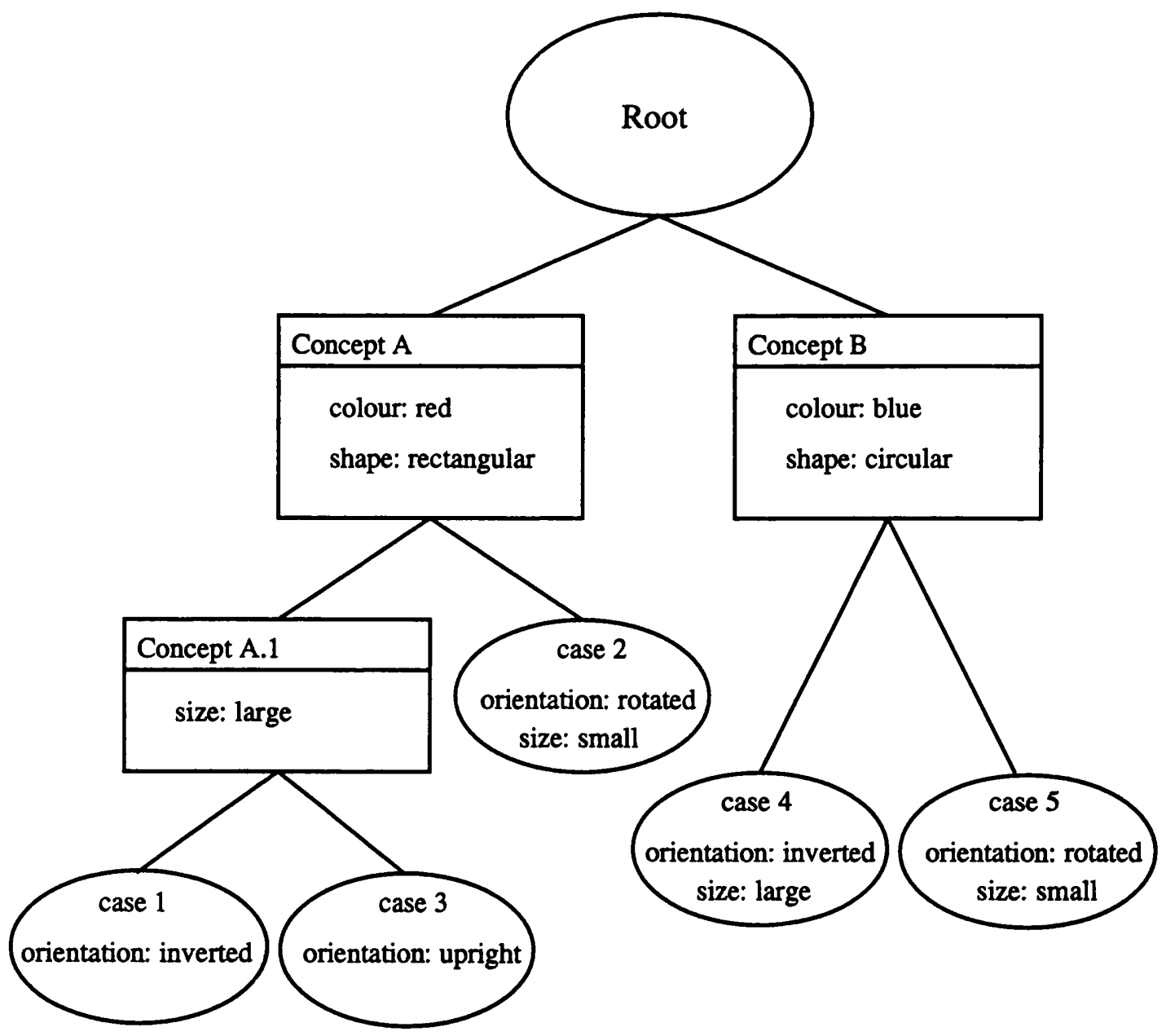

Figure 4.1: A sample discrimination hierarchy. 


\begin{tabular}{|l|l|}
\hline Instance & Features \\
\hline Case 1 & $\begin{array}{l}\text { colour: red, shape: rectangular } \\
\text { size: large, orientation: inverted }\end{array}$ \\
\hline Case 2 & $\begin{array}{l}\text { colour: red, shape: rectangular } \\
\text { size: small, orientation: rotated }\end{array}$ \\
\hline Case 3 & $\begin{array}{l}\text { colour: red, shape: rectangular } \\
\text { size: large, orientation: upright }\end{array}$ \\
\hline Case 4 & $\begin{array}{l}\text { colour: blue, shape: circular } \\
\text { size: large, orientation: inverted }\end{array}$ \\
\hline Case 5 & $\begin{array}{l}\text { colour: blue, shape: circular } \\
\text { size: small, orientation: rotated }\end{array}$ \\
\hline
\end{tabular}

Table 4.1: Five objects defined by attributes: colour, shape, size and orientation.

size and orientation), are located and constructed within a discrimination network. The instance descriptions are shown in Table 4.1 (which lists the cases in the order in which they are presented to a discrimination forming algorithm). The top node in the hierarchy is called the root. The root is the point at which search commences downwards through the network in order to find the most applicable location for a specific instance. Instances stored at the root node represent unclassified cases. Child nodes that represent concepts are always matched with the input case first followed by the instances stored at the node. For example, when the first case of Table 4.1 is entered to the network (which consists only of the root), there are no child nodes of the root with which to match the case. Thus, Case 1 is initially stored as an instance of the root and is unclassified. When Case 2 is entered, again all child concepts are checked first, but none exist so the instances stored at the root are checked (and the only instance stored at the root is Case 1). Case 1 and Case 2 match on two features: colour red and shape rectangular. Concept $A$ is thus created with an associated description consisting of these two features. Two cases are then created and stored as children of Concept $A$ in terms of the distinctive features of Case 1 and Case 2 (Case 1 is stored with the features size large and orientation inverted, and Case 2 is created with the features size small and orientation rotated). As Case 1 is now classified, it is removed as a child of the root. When Case 3 enters the network, it is compared to the one child node now stored at the root, Concept A. Case 3 matches Concept $\mathrm{A}$ on both its listed features and is subsequently matched with the children of Concept A, which at this time comprises two instances: Case 1 and Case 2. When these instances are matched with Case 3, they are compared along the distinctive features of Case 3 in relation to the parent node. That is to say, the distinctive features 
of Case 3 when it successfully matches Concept $A$ are: size large and orientation upright. These two distinctive features are compared with the attribute-value pairs listed on Cases 1 and 2. Cases 2 and 3 do not match on any features, but Case 1 matches Case 3 on the feature size large. A new child node is created, called Concept A.1, as a child of Concept $A$ with this distinguishing feature. Subsequently, Case 1 and Case 3 are stored below Concept A.1 in terms of their distinctive features (Case 1 is stored as orientation inverted and Case 3 with the feature orientation upright). Case 1 has now been re-classified and so is removed as a child of Concept A. An important result of this procedure is that the relevant cases are not stored in terms of all their features. For example, Cases 1 and 3 are only stored in terms of one attribute-value pair, although they are recorded (in Table 4.1) in terms of four features. This is a key facet of discrimination networks. The entire case description is derived through top-down search through the network. In this sense, nodes inherit the features of ancestor nodes. For example, whilst Case 1 only lists one feature, its full description is derived from the two concept nodes under which it is classified, Concept $A$ and Concept A.1.

With the same procedure as described above, when Case 4 enters the network it fails to match Concept $A$ and is stored as an instance of the root. When Case $\mathbf{5}$ is entered, it is first checked against Concept $A$, which it fails to match, and is then compared to Case 4. Cases 4 and 5 match on two features: colour blue and shape circular. Thus, a new concept called Concept $B$ is created and stored at the root, and Cases 4 and 5 are stored below it in terms of their distinctive features.

\subsection{The UNIMEM Algorithm}

UNIMEM utilises the discriminative indexing approach described in the previous section. The basic algorithm is shown in Figures 4.2 and 4.3. When a case, or instance $(\mathrm{I})$, arrives at a node $(\mathrm{N})$ the similarity between the case and $\mathrm{N}$ is evaluated in terms of the conjunction of features that match $(H)$. If this evaluation succeeds, i.e., if sufficient similarity between the object case and the node is deemed to exist, then sorting proceeds to each of the relevant sub-nodes, or children (C), and subsequent comparison is based on the features that did not match with the node (K). This idea is particularly relevant to Schank's premise that events are indexed through discriminating features (episodes are remembered, or triggered, by the failure of an expectation [65]). Kolodner's imple- 
Input: The current node $\mathrm{N}$ in the network.

An unclassified instance I.

The set of I's features F.

Variables: $\mathrm{N}$ and $\mathrm{C}$ are nodes in the network.

G, $H$ and $K$ are sets of features (attribute values).

$\mathrm{J}$ is an instance stored at a node.

$S$ is a list of nodes.

Unimem(N, I, F)

Let $\mathbf{G}$ be the set of features stored on $\mathbf{N}$.

Let $\mathrm{H}$ be the features in $\mathrm{F}$ that match features in $\mathrm{G}$.

Let $\mathrm{K}$ be the features in $\mathrm{F}$ that do not match features in $\mathrm{G}$.

If $\mathrm{N}$ is not the root node,

Then If there are too few features in $\mathrm{H}$, or Evaluate(N, H, K) returns TRUE,

Then return the empty list.

Let $\mathrm{S}$ be the empty list.

For each child $\mathrm{C}$ of node $\mathrm{N}$,

If $C$ is indexed by a feature in $K$,

Then let $\mathrm{S}$ be Union(S, Unimem( $\mathrm{C}, \mathrm{I}, \mathrm{K})$ ).

If $\mathrm{S}$ is the empty list,

Then for each instance $J$ at node $N$,

Let $\mathrm{S}$ be Union(S, Generalise(N, J, I, F)).

If $S$ is the empty list,

Then store $\mathrm{I}$ as an instance of node $\mathrm{N}$ with features $\mathrm{K}$.

Return N

Figure 4.2: The top level UNIMEM algorithm.

mentation of these ideas, CYRUS [42, 43], is also based on discriminative indexing. The resulting network structure is organised such that a node $\mathbf{N}$ is described in terms of a conjunction of features that are exhibited by instances stored below it, which are in turn defined by the features that differ to those on $\mathrm{N}$. Thus, an individual case is not constructed in terms of all the features that it exhibits ( $a$ list of features is read by UNIMEM for the new case, but the case is not actually created and stored in the network until its location is decided). The complete set of features for an individual case must, therefore, be derived through top-down search through the network.

Sorting, or search, is effectively controlled at the point at which a case is evaluated with a node. UNIMEM refers to a system parameter (an integer value) to determine if the two objects match on enough features in order to proceed to the child nodes (C) linked to N. This is an important facet of UNIMEM, particularly with respect to the 
Variables: $\mathrm{N}$ and $\mathrm{C}$ are nodes in the network.

F, G, $\mathrm{H}$ and $\mathrm{K}$ are sets of features (attribute values).

$I$ and $J$ are the names of instances.

$\mathrm{S}$ and $\mathrm{T}$ are predictability scores of nodes' features.

Generalise(N, J, I, F)

Let $\mathrm{G}$ be the features in instance $\mathrm{J}$.

Let $\mathrm{H}$ be the features in $\mathrm{F}$ that match features in $\mathrm{G}$.

Let $\mathrm{K}$ be the features in $\mathrm{F}$ that do not match features in $\mathrm{G}$.

If $\mathrm{H}$ contains enough features,

Then create a new child $\mathrm{C}$ of node $\mathrm{N}$ with features $\mathrm{H}$.

Remove $\mathrm{J}$ as an instance of $\mathrm{N}$.

Let $\mathrm{G}^{\prime}$ be the features in $\mathrm{G}$ that are not in $\mathrm{H}$.

Let $F^{\prime}$ be the features in $F$ that are not in $\mathrm{H}$.

Store $\mathrm{J}$ as an instance of $\mathrm{C}$ with features $\mathrm{G}^{\prime}$.

Store $\mathrm{I}$ as an instance of $\mathrm{C}$ with features $\mathrm{F}^{\prime}$.

Return C.

Evaluate(N, H, K)

For each nonpermanent feature $\mathrm{F}$ in $\mathrm{H}$,

Raise the predictability score $\mathrm{S}$ for $\mathrm{F}$ on $\mathrm{N}$.

If $\mathrm{S}$ is high enough,

Then make $F$ a permanent feature of $N$.

For each nonpermanent feature $\mathrm{G}$ in $\mathrm{K}$,

Lower the predictability score $\mathrm{T}$ for $\mathrm{G}$ on $\mathrm{N}$.

If $T$ is low enough,

Then remove feature $\mathrm{G}$ from $\mathrm{N}$.

If $\mathrm{N}$ has too few features,

Then remove $\mathrm{N}$ from its parent's list of children.

Return TRUE.

Return FALSE.

Figure 4.3: The main control functions in UNIMEM.

dysmorphology domain. The input case is not required to overlap on all features defined at the node $\mathrm{N}$. It is required only to match on enough features so that similarity between the two objects is deemed sufficient in order for search to proceed to sub-nodes of $\mathrm{N}$. Thus, UNIMEM adopts a polymorphic approach to matching that is compatible with the type of similarity assessment performed in dysmorphology. Search is thus confined to those nodes and sub-nodes that evaluate sufficiently with the object case. This procedure is sometimes referred to as concept refinement ${ }^{2}$. The evaluate function (see Figure 4.3) will also confine search. Each feature defined on each node has an associated score which

\footnotetext{
${ }^{2}$ This type of sorting procedure is also referred to as beam search.
} 
represents the predictability of that feature. The predictability of a feature is related to its incidence with respect to the instances stored at a particular concept node. On evaluation, the predictability of each feature listed by the concept node is incremented or decremented depending on whether there is a match or not. If the input case has a feature listed on a concept node, then the predictability of that feature is incremented (on the node). Conversely, its predictability is decremented if the new case does not have the feature. If the resulting predictability score for a feature reaches a predefined threshold (another system parameter), then that feature is made permanent on $\mathrm{N}$. That is to say, the feature is fixed to the node so that in future comparisons its predictability score will not be altered. This is an informal way of deriving the confidence of a specific feature with respect to a concept, i.e., the feature is sufficiently frequent among the class members that it may be considered predictable for the class [50]. Similarly, if the predictability score for a feature falls below a predefined threshold, it will be removed from the node description (its frequency being too low to imply predictability). Future searches will use permanent features to index nodes with respect to incoming cases. However, as the associated predictability score of a permanent feature cannot be decremented, the feature cannot ultimately be removed from concept. These operations involving predictability levels control how UNIMEM forms concepts and updates its category information. A further ramification is that if the node develops too few features (determined by a system parameter) it is effectively removed from the network (considered to be too general to form a valid concept). This procedure is sometimes referred to as pruning. In such an instance, the evaluate function confines search as its associated child nodes will not be considered (they are also effectively removed from the network).

Once the object case (I) can proceed no further, or deeper, in the current branch (i.e., it does not evaluate sufficiently with any of the child nodes of $N$ ), it is compared to each of the instances $(\mathrm{J})$ stored at $\mathrm{N}$. The generalise function shown in Figure 4.3 is invoked in order to generate a child of $\mathrm{N}$ based on the overlapping features of $\mathrm{I}$ and $\mathrm{J}$. Another system parameter (an integer count) is used to determine if there are enough matching features $(\mathrm{H})$ to do this. If so, a new child node $(\mathrm{C})$ is defined and created by the set of features $\mathrm{H}$, and the object case $\mathrm{I}$ is created and stored below $\mathrm{C}$ in terms of the features that differ to those on $C\left(F^{\prime}\right)$. Similarly, the previous instance (J) is now stored below $C$ in terms of the features on it that differ ( $\left.G^{\prime}\right)$.

Another important aspect of UNIMEM is that it forms graph networks rather than 
hierarchical trees. That is to say, an input case (I), will be stored at all the (deepest) nodes at which it has proved sufficiently similar. This is an important consideration with respect to the dysmorphology domain. An individual case may exhibit more than one disease. Furthermore, a case may not achieve a firm diagnosis, and may subsequently be (tentatively) categorised against more than one possible syndrome.

\subsection{Experiments with UNIMEM}

The UNIMEM algorithm has been applied to experimental data from three sources: the London Dysmorphology Database, the Skeletal Dysplasias Registry, and the Human Cytogenetics Database. Each data set consists of a number of individual case descriptions. These correspond to actual patients that have been examined and documented in terms of dysmorphic features, and which have in turn been translated to the relevant computer representation. The first two groups span cases that have a diagnosis across related syndromes, or syndrome families. This is intended to allow an evaluation of how the CBL algorithm performs when matching cases and in forming conceptual categories. Cases from the Human Cytogenetics Database are labelled according to a chromosomal abnormality rather than a syndrome as such. However, they too form families, or groups, based on the variation of defects categorised below a primary chromosome disorder.

\subsubsection{Acrocephalosyndactyly Data Set}

The first data set comprises twenty-eight cases from the London Dysmorphology Database (see Table 4.2). Their diagnoses correspond to three variations of Acrocephalosyndactyly $^{3}$ (Saethre-Chotzen, Apert and Pfeiffer syndromes), along with a further three related conditions: Treacher-Collins syndrome (also referred to as Mandibulofacial Dysostosis 4 , Nager Acrofacial Dysostosis and Acrofacial Dysostosis with post-axial defects, i.e., facial dysostosis in combination with a limb defect (sometimes referred to as Millar syndrome [30]). Cases are described by a list of dysmorphic features as denoted by the three-level LDDB representation described in Chapter 2. For use in these experiments, features have been translated to equivalent symbolic triplets of the form: (clinical-region attribute value). Table 4.3 shows the equivalent descriptions for

\footnotetext{
${ }^{3}$ Smith refers to these as craniosynostosis syndromes

'Defective ossification (hypoplasia) of the facial bones in particular.
} 
Case 7877 (diagnosed as Saethre-Chotzen syndrome).

\subsubsection{Skeletal Dysplasias Data Set}

The second data set originates from the Skeletal Dysplasias Registry (SDR) and comprises twenty-seven cases diagnosed across nine syndromes. These nine syndromes are further categorised into three families (see Table 4.4): the Chondrodysplasia group (Thanatophoric dysplasia, Achondroplasia and Hypochondroplasia), characterised by growth deficiencies with short limbs and spine abnormalities, the short-rib group (SRP I/III, SRP III and Asphyxiating Thoracic Dystrophy, also called Jeune Thoracic Dystrophy), and a third group linked by abnormalities of the long bones and vertebrae (SEDC, SEMD and Achondrogenesis II). The case representation utilised by SDR is not unlike that employed in LDDB although the regional components are naturally specific to radiology. The radiological regions defined within the SDR representation are shown in Table 4.5. As with the LDDB data set, for the purpose of these experiments features are described in terms of symbolic triplets consisting of the radiological region, a sub-category and an an associated value, e.g., (chest rib-shortening severe).

\subsubsection{Chromosomal Abnormality Test Cases}

Two additional data sets have been made available from the Human Cytogenetics Database (HCDB), developed by Dr Albert Schinzel ${ }^{5}$. This database has similarities to LDDB $^{6}$ and stores clinical and cytogenetic data relating to chromosome aberrations. More specifically, HCDB lists over 5000 individual cases with multiple malformations, each of which has a specific chromosomal abnormality.

There are 23 pairs of chromosomes ( 23 from each parent). The first 22 are known as the autosomes and are numbered 1-22. The remaining pair consists of the sex chromosomes. A chromosome is divided into long and short arms (called the $q$ and $p$ arms respectively) by a specialised area of the chromosome known as the centromere. Within each arm individual bands are visible through a special staining technique when a karyotype is performed. Bands are numbered from the centromere distally. Figure 4.4 shows

\footnotetext{
${ }^{5}$ Dr. Schinzel is currently Associate Professor at the Institute of Medical Genetics in Zürich, Switzerland.

'The Human Cytogenetics Database is part of a suite of databases edited by Drs. Winter and Baraitser which have the same underlying architecture.
} 


\begin{tabular}{|l|l|}
\hline SYNDROME & CASE IDENTIFIERS \\
\hline Apert, Acrocephalosyndactyly Type I & $6761,616929,618717,606067,564525$ \\
Saethre-Chotzen, Acrocephalosyndactyly Type III & $5462,7464,6150 \mathrm{a}, 7385,7877$ \\
Pfeiffer, Acrocephalosyndactyly Type V & $0001,5286 \mathrm{a}, 5286 \mathrm{~b}, 5286 \mathrm{c}, 4200$ \\
\hline Nager Acrofacial Dysostosis & $\mathbf{8 1 7 5 , 6 5 6 6 , 7 0 3 1 , 0 0 0 5 , 5 7 5 4}$ \\
Treacher Collins Syndrome & $6638,6351,8967,7312$ \\
Acrofacial Dysostosis with Post-Axial Defects & $0002,0003,0004,9605$ \\
\hline
\end{tabular}

Table 4.2: Acrocephalosyndactyly and Acrofacial Dysostoses data set.

\begin{tabular}{|ll|}
\hline LDDB Code & Description \\
\hline $03-01-06$ & (cranium general plagiocephaly/asymmetrical-skull) \\
$03-07-01$ & (cranium cranial-sutures craniosynostosis) \\
$05-01-03$ & (forehead prominent frontal-bossing) \\
$05-01-02$ & (forehead prominent high-frontal-hairline) \\
$06-05-03$ & (ears ear-crus prominent-ear-crus) \\
$07-01-05$ & (eyes general hypertelorism) \\
$07-13-08$ & (eyes vision strabismus/gaze-palsy) \\
$07-13-03$ & (eyes vision hypermetropia) \\
$10-06-02$ & (face maxilla hypoplastic-maxilla-excluding-malar-region) \\
$32-01-20$ & (neurology general mental-retardation) \\
$32-01-12$ & (neurology general hypotonia) \\
\hline
\end{tabular}

Table 4.3: Symbolic feature descriptions for Case 7877.

\begin{tabular}{|l|l|}
\hline SYNDROME & CASE IDENTIFIERS \\
\hline Achondroplasia & $091-0251,090-0197,090-0258$ \\
Thanatophoric Dysplasia without Kleeblattschadel & $091-0161,092-0054,091-0222$ \\
Hypochondroplasia & $091-0176,092-0167,089-0052$ \\
\hline Asphyxiating Thoracic Dystrophy & $090-0050,092-0080,088-0116$ \\
Short Rib Polydactyly Type I/III (Saldino-Noonan) (Naumoft) & $089-0135,090-0009,091-0250$ \\
Short Rib Polydactyly Type II (Majewski) & $090-0225$ \\
Short Rib Polydactyly Type II (Majewski) - Tentative) & $089-0180,091-0156$ \\
\hline Spondyloepiphyseal Dysplasia Congenita (SEDC) & $091-0084,091-0185,092-0181$ \\
Spondyloepimetaphyseal Dysplasia Strudwick Type (SEMD) & $092-0184,091-0162,091-0096$ \\
Achondrogenesis II/Hypochondrogenesis & $091-0215,091-0035,090-0122$ \\
\hline
\end{tabular}

Table 4.4: Skeletal dysplasias data set. 


\begin{tabular}{|lll|}
\hline bone-density & long-bone-size & fractures \\
spine-and-vertebrae & skull-and-face & pelvis \\
chest & hands & feet \\
long-bones & humerus & elbow \\
radius & ulna & femur \\
tibia & fibular-overgrowth & fibula \\
hips & knees & patella \\
lesions-in-bone & acheiropodia & \\
bone-age & bone-exostoses-and-enchondromas & \\
\hline
\end{tabular}

Table 4.5: Radiological items represented in SDR.

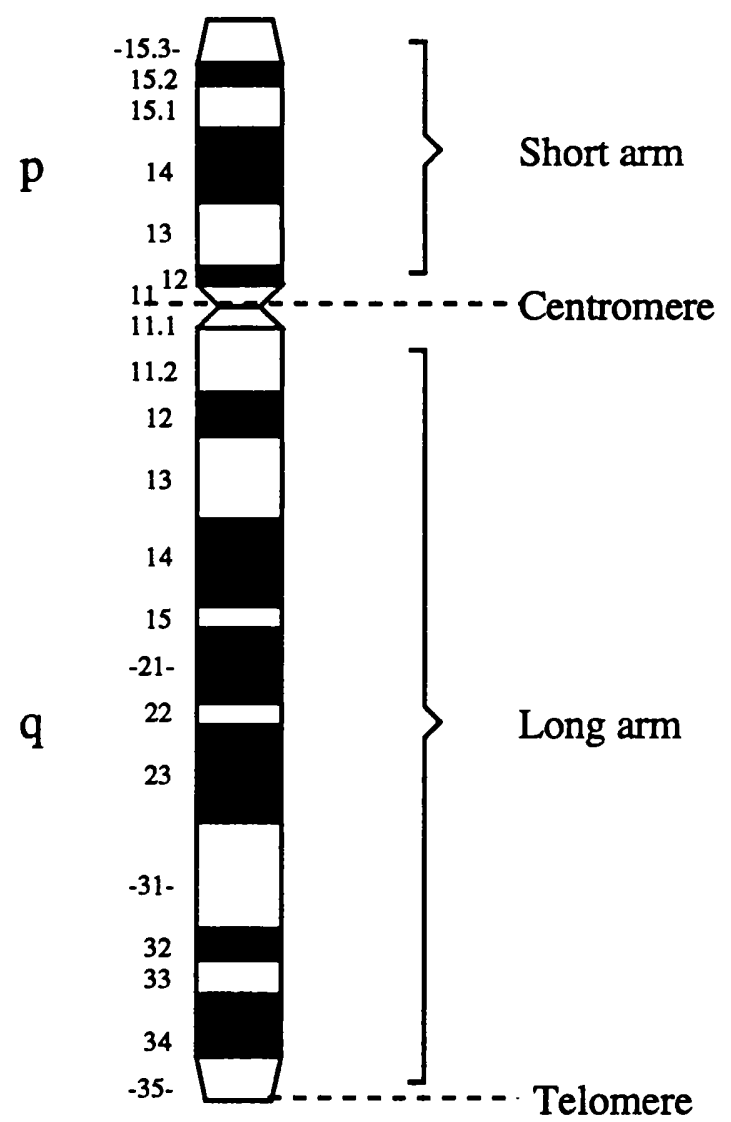

Figure 4.4: Idiogram of the number 5 chromosome. 
a schematic diagram (or idiogram) of the number 5 chromosome.

Chapter 1 associated Down syndrome with the chromosomal disorder known as trisomy of chromosome 21 . This is an example of an abnormality of chromosome number. In Down syndrome there are 47 chromosomes instead of 46 (the extra chromosome being an additional 21 chromosome). In addition to abnormalities of chromosome number, individual chromosomes can be broken and re-arranged in such a way that their structure is altered. The test cases made available in this instance demonstrate such structural abnormalities.

Several different types of structural re-arrangement are possible and these are indicated by a nomenclature based on cytogenetic shorthand. For example, part of a chromosome may be duplicated, in which case, the duplicated segment is denoted in the shorthand. The descriptor $\operatorname{dup}(5)$ ( $q 33 \rightarrow q$ ter) denotes that chromosome number 5 has a duplication in the long arm, and the duplicated segment is the section from band 33 to the telomere (the end of the arm), and the duplicate segment retains the same orientation with respect to the centromere ${ }^{7}$. Another example is del(2) (q24 $\rightarrow$ q31), which is cytogenetic shorthand for a deletion in the number 2 chromosome on the long arm between bands 24 and 31 .

The cases of multiple malformations listed in HCDB are described using the same master list of dysmorphic features that has been developed for LDDB. Furthermore, it is thought that cases with chromosomal abnormalities may also be grouped according to overlapping malformation patterns. That is to say, as with non-chromosomal syndromes, cases with the same chromosomal abnormality are expected to exhibit a similar set of dysmorphic features. Two data sets consisting of cases with structural chromosomal disorders have been used in the experiments with the UNIMEM algorithm. The first set contains 77 cases with a duplication in the number 5 chromosome. Of these cases, those with the same specific banding defect (e.g., (q33 $\rightarrow$ qter)) are expected to show some overlap in terms of their pattern of anomalies. The 77 cases comprise a number of different banding defects. In a sense, it could be said that dup(5) denotes the family, whilst the various banding defects denote distinct syndrome-like entities within it. Similarly, the second set contains 72 cases with a deletion in the number 2 chromosome.

\footnotetext{
${ }^{7}$ The full shorthand includes the total number of chromosomes and the sex chromosomes. For a male with the structural defect described, but with inverted orientation the shorthand would be $46 X Y$ inv $\operatorname{dup}(5)$ (q33 $\rightarrow q$ ter).
} 


\subsubsection{Results}

An accurate reconstruction of the UNIMEM algorithm as described in the literature [50], and shown in Figures 4.2 and 4.3, has been implemented in Common Lisp. This algorithm was utilised with each of the four instance sets from LDDB, SDD and HCDB. Individual cases were presented incrementally to the top level function. The following sections report how the algorithm performed with respect to the different data sets. The ensuing discussion assesses the strengths and weaknesses of the approach with reference to the dysmorphology domain.

\section{Acrocephalosyndactyly and Skeletal Dysplasias Data Sets}

The concept hierarchies generated by the Acrocephalosyndactyly (LDDB) data set and the skeletal dysplasias (SDR) data set are shown in Figures 4.5 and 4.6 respectively. Referring to Figure 4.5 (Network A.1), twenty-four cases (of a total of twenty-eight) have been integrated into a classification network which comprises eleven concept nodes. The remaining four uncategorised cases have been stored at the root node (R). Table 4.6 lists the descriptions that have been generated at each node for Network A.1. Three nodes list permanent features for the respective concepts: N7, N8 and N9. The existence of permanent features on a node, which are established through incidence among the stored cases, implies (albeit in an informal manner) a degree of confidence in the predictability of these features for the node, i.e., they are prototypical for the node. Node N7 has successfully grouped all cases of Acrofacial Dysostosis with post-axial defects. All six features listed as permanent on Node N7, as well as the one non-permanent feature (ectropion of the eyelids ${ }^{8}$ ) are listed as principal features of Acrofacial Dysostosis in Smith's compendium. Key features listed in Smith's book that are not evident in the concept description are: coloboma of the eyelids, hypoplastic ears, syndactyly of the digits, and accessory nipples. However, these anomalies are actually exhibited at least once amongst the cases stored at Node N7 (but not frequent enough to merit being made permanent).

Nodes N8 and N9 (including the sub-nodes N8.1 and N9.1) collectively group cases of Acrocephalosyndactyly. Craniosynostosis is listed in both these node descriptions. This would be expected of a concept that links cases of Acrocephalosyndactyly, as craniosyn-

\footnotetext{
An eversion of the eyelids.
} 


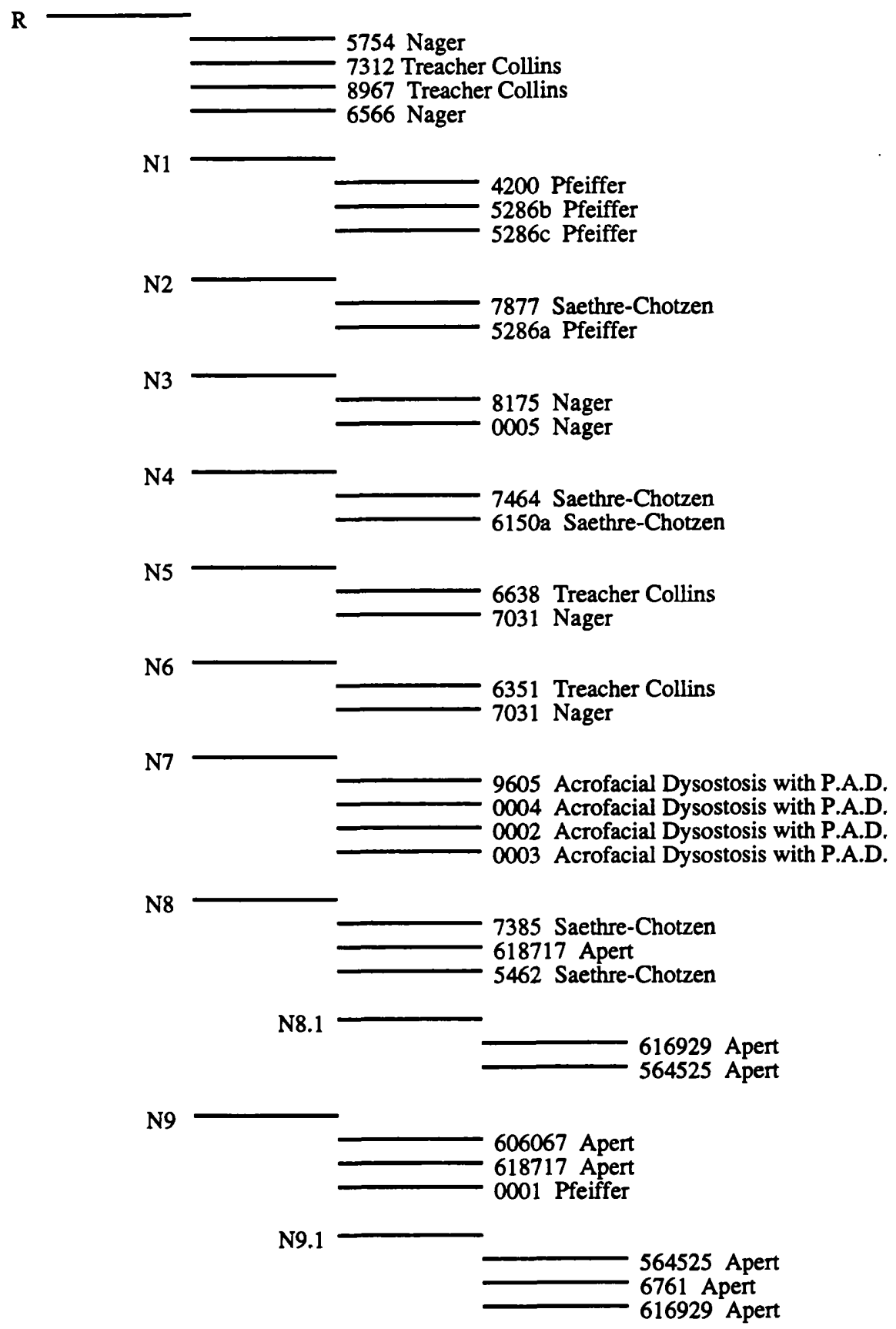

Figure 4.5: Network A.1: Concept hierarchy generated by the Acrocephalosyndactyly data set. 
$\mathbf{R}$

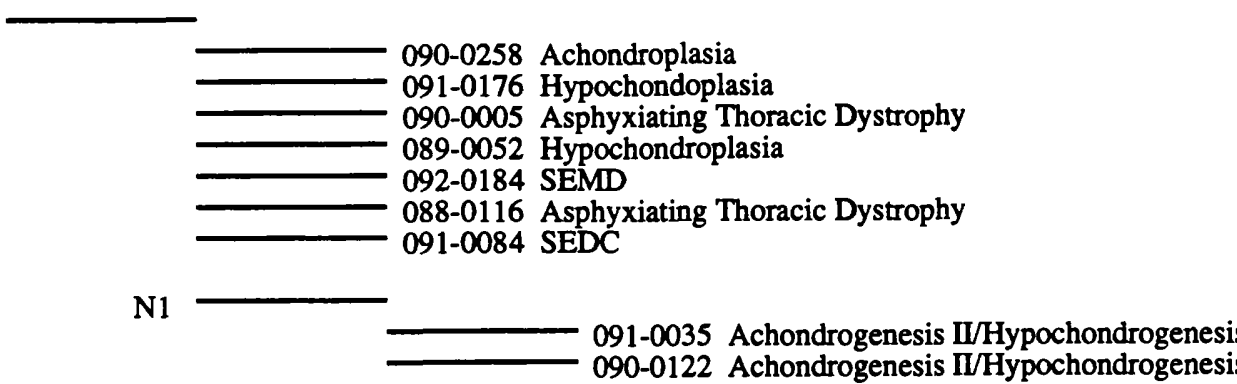

N2

090-0122 Achondrogenesis I/Hypochondrogenesis

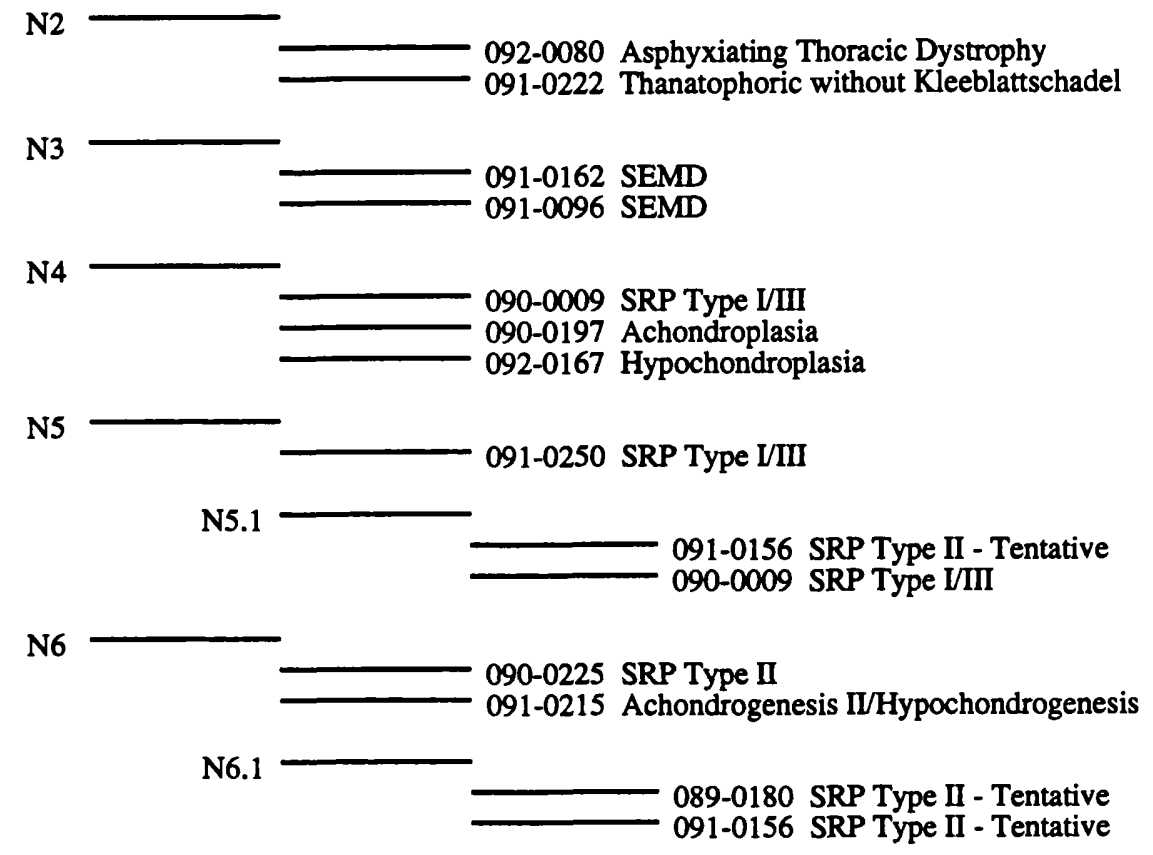

N7

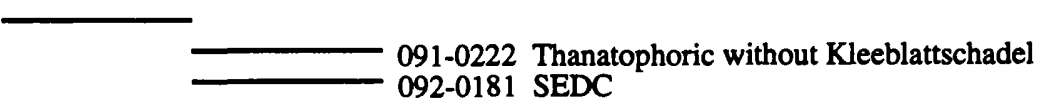

N7.1

091-0251 Achondroplasia

090-0197 Achondroplasia

N8

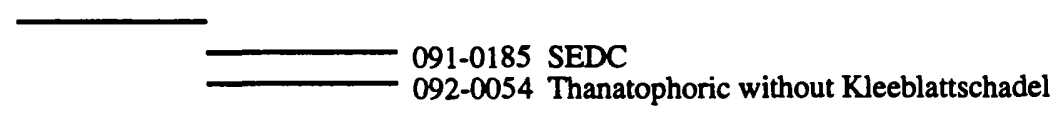

N9

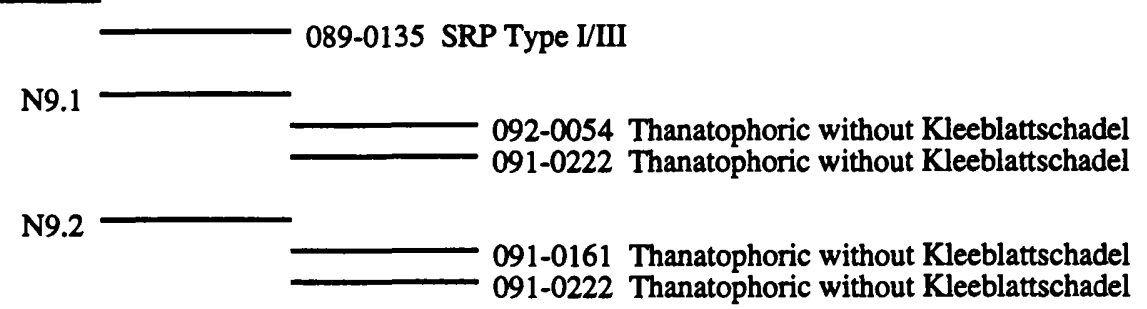

Figure 4.6: Network S.1: Concept hierarchy generated by the skeletal dysplasias data set. 


\begin{tabular}{|c|c|c|}
\hline Node & Permanent & Non-permanent \\
\hline N1 & & 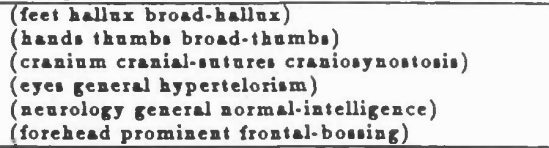 \\
\hline N2 & & 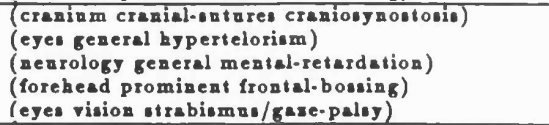 \\
\hline N3 & & 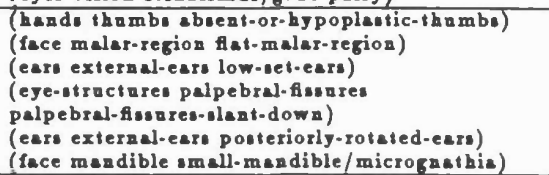 \\
\hline N4 & & 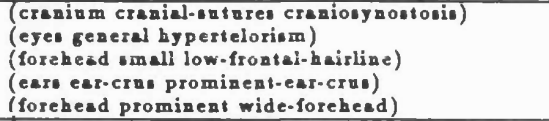 \\
\hline No & & 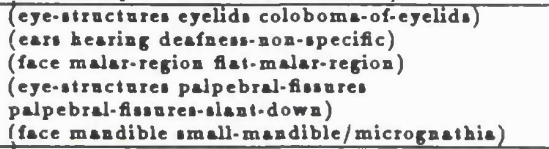 \\
\hline N6 & & 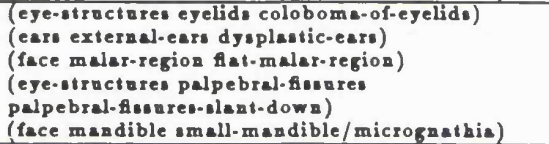 \\
\hline N 7 & $\begin{array}{l}\text { (feet toes absent-toes) } \\
\text { (handofingert absent-fingers-or-oligodactyly) } \\
\text { (upper-limbs forearm hypoplastic-or-absent-alas) } \\
\text { (oral-region palate cleft-palate) } \\
\text { (face mandible small-mandible/micrognathia) } \\
\text { (face malar-region fat-malar-segion) }\end{array}$ & (eye-biractures eyelido ectropion-of-eyelido) \\
\hline No & 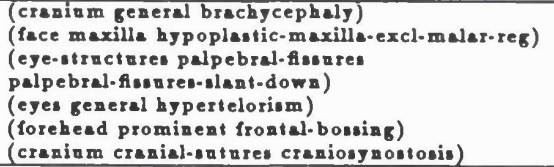 & \\
\hline N8.1 & & 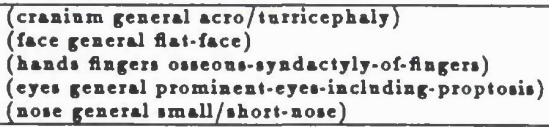 \\
\hline No & 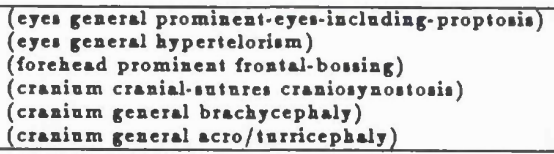 & \\
\hline No.1 & & 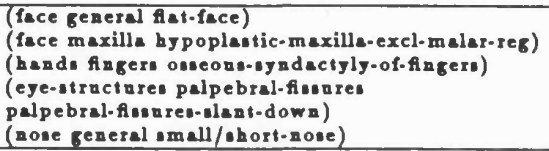 \\
\hline
\end{tabular}

Table 4.6: Node descriptions for Network A.1. 
ostosis is a key hard anomaly that is known to exist in all the variations of this syndrome family. Recalling Figure 2.6, which is derived from the London Dysmorphology Database, it can be seen that a key feature distinguishing Apert type Acrocephalosyndactyly from the other two syndromes is acro/turricephaly ${ }^{9}$. This is reflected in the relative descriptions of N8 and N9, and also helps to distinguish the two cases of Apert that have formed Node N8.1. Overall, cases that would be expected to be grouped together are (i.e., cases from the same higher order family as shown in Table 4.2). Cases of the three Acrocephalosyndactyly syndromes are grouped across Nodes N1, N2, N4, N8 and N9, whilst cases of Nager, Treacher-Collins and Acrofacial Dysostoses with post-axial defects are linked by nodes N3, N5, N6 and N7.

Referring now to Figure 4.6, overall one can interpret a weaker performance by UNIMEM with respect to the concept hierarchy that has been formed. Seven cases remain unclassified (and are stored at the root) and the remainder are grouped across fourteen concept nodes. Three nodes list permanent features in their respective concept descriptions: N6, N7 and N9. However, when comparing the actual diagnoses of cases stored at these nodes it can be seen that the grouping is inconsistent with the known family links between syndromes that are shown in Table 4.4. Whilst Network A.1 succeeds in grouping together cases of the same syndrome family, Network S.1 links cases from different dysplasia families. For example, Node N7 links an SEDC case with a case of Thanatophoric dysplasia. Referring to Table 4.4 it can be seen that these syndromes are from different higher order families.

Because the available data sets comprise cases of known diagnoses the relative performance of UNIMEM with respect to the different data sets can be represented in terms of the accuracy in which it groups cases. Figure 4.7 charts the mean group accuracy factor $(\overline{G A F})$ for each of the four data sets when used with the UNIMEM program. The group accuracy factor $(G A F)$ reflects the combined accuracy for each diagnostic pairing on a concept node that stores two or more cases. A diagnostic pairing corresponds to a comparison of the actual diagnoses between two individual cases that are stored at the same concept node. Each case at a node is compared with all other cases stored at the same node. Thus, for a node with $n$ stored cases there will be a total of $(n-1)$ ! pairings. Each diagnostic pairing $\left(P_{i j}\right)$ is assigned a value which corresponds to one of three possible conditions:

\footnotetext{
${ }^{\circ} \mathrm{A}$ high and peaked/pointed skull.
} 
1. Both cases ( $i$ and $j$ ) have the same actual diagnosis.

2. The actual diagnoses for $i$ and $j$ are different and belong to different syndrome families.

3. The actual diagnoses of $i$ and $j$ are different, but belong to the same syndrome family.

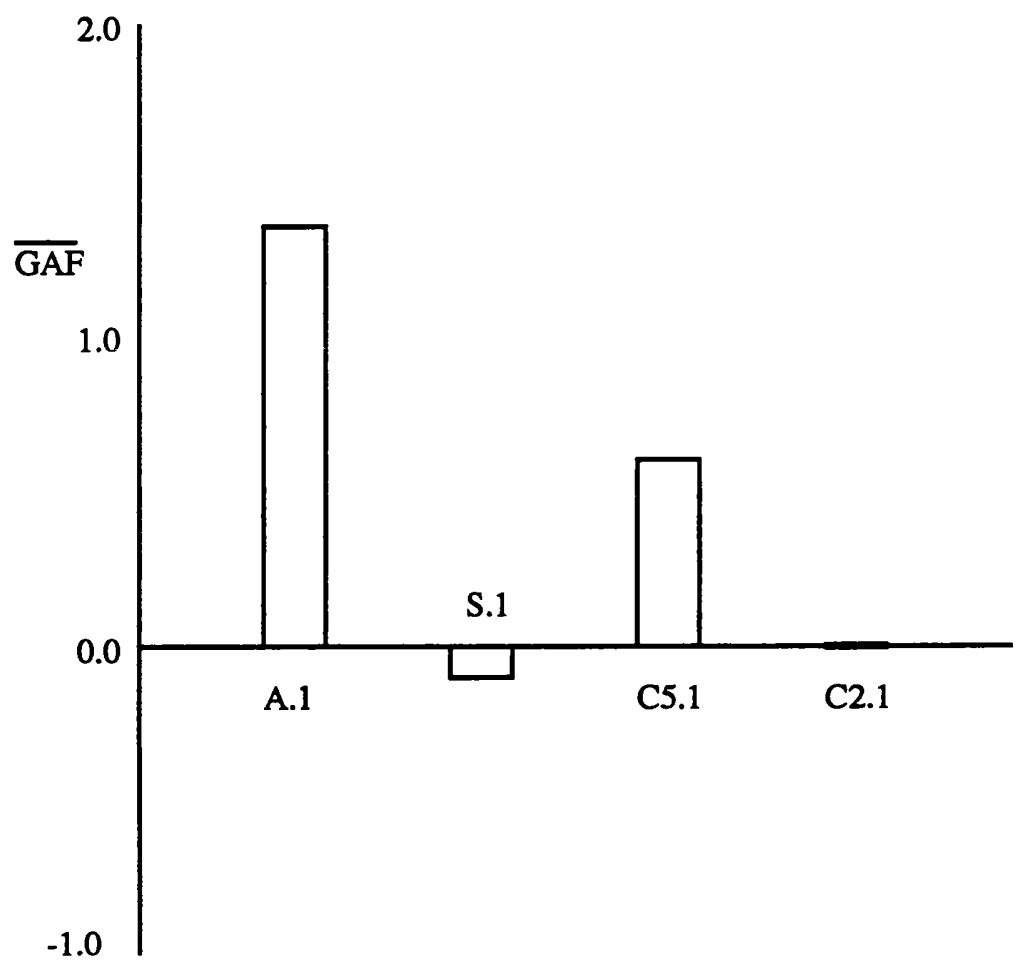

KEY:

Concept Networks Generated with Data Sets:
A.1 Acrocephalosyndactyly Data Set
(LDDB)
S.1 Skeletal Dysplasias Data Set
(SDR)
C5.1 HCDB Data Set 1
(dup5)
C2.1 HCDB Data Set 2
(del2)

Figure 4.7: Mean GAF achieved by UNIMEM.

The group accuracy factor of a node is then calculated as the sum of each diagnostic 
pairing across the node such that:

$$
G A F=\sum P_{i j} \quad P_{i j}= \begin{cases}1.0 & \text { if } i=j \\ -1.0 & \text { if } i \neq j \\ -0.25 & \text { if } i \neq j, \text { but } i \wedge j \in \mathcal{F}\end{cases}
$$

The mean group accuracy factor is then given by:

$$
\overline{G A F}=\frac{\sum_{i=1}^{n} G A F_{n}}{n}
$$

where $\mathcal{F}$ is a syndrome family, and $n$ is the number of concept nodes in the network.

Figure 4.7 confirms that UNIMEM performs better, in terms of the average group accuracy, with the LDDB data set compared to the data set from SDR. The mean GAF for concept nodes in Network A.1 is +1.38 , compared to -0.125 for Network S.1. This

\section{Addendum}

The Group Accuracy Factor (GAF) has been created for this work as a measure for specific use with learning algorithms such as UNIMEM in domains in which data sets have already been categorised. Furthermore, the GAF is designed to account for inter-class and intraclass linkages between grouped cases based on what is actually known to be their 'correct' classification.

to propagate to relevant chud nodes of $\mathbf{N}$, when determining it a concept teature 18 frequent enough across the stored cases in order to make it permanent at the relevant node, and in establishing sufficient similarity between two cases in order to create a new concept node. The corresponding parameters that control these processes are integer counts. The literature does not provide any recommendations for these levels. Rather, they are noted as empirical thresholds. This makes sense when data sets can vary in the size of the case description, especially with regard to the thresholds that determine evaluation and generalisation. A generalisation threshold (i.e., the level, or count of matching features that determines that a new concept node should be created) may be set to a level that performs well with one data set. However, if it is used with a different data set that comprises cases with much larger descriptions (i.e., larger lists of features) then generalisation, i.e., the creation of new concepts, may be far more frequent and 
result in a much larger concept hierarchy (with much greater branching). A relatively low evaluation threshold will also be detrimental to algorithmic performance with the second data set. The effect in this instance would be that evaluation would succeed too readily. This could cause a case to be linked to too many different concepts, which again increases the size of the network. One facet of incremental concept formation systems, mentioned in Chapter 2, is that they are designed to operate unsupervised. They are not guided in determining the size or number of concepts within the hierarchy. However, with UNIMEM the levels at which these three parameters are set does effectively control the size of the network, along with the construction of prototypical features on each concept. The fact that the diagnoses are known for each data set has allowed the parameters controlling evaluation, generalisation and permanency to be set at levels which reflect the data to which the algorithm is applied. For instance, the network shown in Figure 4.5 has been created with the following parameter settings:

- The Evaluation Threshold $E_{t}$ equal to 5. This count defines the least number of features with which the input case (I) must match the current node (N) in order for search to proceed to the relevant branch, i.e., the point at which the evaluate function would be called (see Figure 4.2).

- The Generalisation Threshold $G_{t}$ equal to 5. This level defines the number of matching features required in order to abstract a new node from the input case and an instance stored within the network.

- The Predictability Threshold $P_{t}$ equal to 3. This number effectively defines how many stored instances must have a particular feature in order for that feature to be made permanent on the parent node description (or concept).

Thus, by adjusting these scores the depth and breadth of the resulting network can be varied along with the concept descriptions (in terms of what features are made permanent). An important consideration, therefore, when choosing these scores is the size of the case descriptions. The mean number of case features listed for the Acrocephalosyndactyly set is 9.21 (maximum $=13$, minimum $=6$ ). This value is 29.29 for the skeletal dysplasias set and is, thus, significantly larger (maxmum $=55$, minimum $=7$ ). The network shown in Figure 4.6 is generated with the values: $E_{t}=13, G_{t}=15$, and $P_{t}=3$. The difference in the mean size of case descriptions between the LDDB set and that 
of SDR reflects a difference in approach when recording individual patient records (and converting to a computerised format). The LDDB cases are described in terms of relatively significant features, whereas the SDR cases incorporate many more occasional features. This factor does have an effect on the performance of UNIMEM which assumes that significance is (merely) proportional to frequency. As nodes are generated only by counts of matching features, without any knowledge of feature salience (diagnosticity), the formation of accurate concepts (with respect to the known diagnoses) becomes more difficult when a case description incorporates many features of low importance. This explains why cases from different syndrome families are grouped together more readily in Network S.1. Because feature diagnosticity is not involved in the evaluation of similarity between cases and concepts, links can be formed between cases based on features of low diagnostic importance. The practical implication of this is that to generate hierarchies of approximately the same breadth and depth of what is known to actually be correct, the system parameters must be adjusted to reflect the granularity of the data set under investigation. Thus, in this sense, the UNIMEM program really is supervised.

\section{Chromosomal Abnormality Data Sets}

The parameter settings for the chromosomal abnormality data sets were kept the same as those for the Acrocephalosyndactyly cases (as cases from HCDB are consistent with the LDDB format). The dup(5) data set generated a total of 31 concept nodes (including 3 sub-nodes) against which 58 individual cases are stored from the total of 77 . Six of these cases are linked to more than one node (see Figure 4.8). The del(2) data set generated a total of 35 concept nodes (including 4 sub-nodes), with 57 of the 72 cases linked to concept nodes (4 cases are located at more than one node). This network is shown by Figure 4.9.

The majority of nodes within both these concept networks have been formed by two matching cases, and so do not have permanent features listed in the concept description. Thus, it may be concluded that, overall, these concepts are generally weaker than those generated with the non-chromosomal cases. However, the networks generated with the UNIMEM algorithm suggest some linkage between cases with the same chromosome banding defect. In Network C5.1 (Figure 4.8) Node N21 stores four cases denoted by the banding defect ( $q 33 \rightarrow q$ ter), and has a child node (N21.1) formed by 2 cases, one of which is also denoted by this specific chromosomal abnormality. The description of 
$\mathbf{R}$

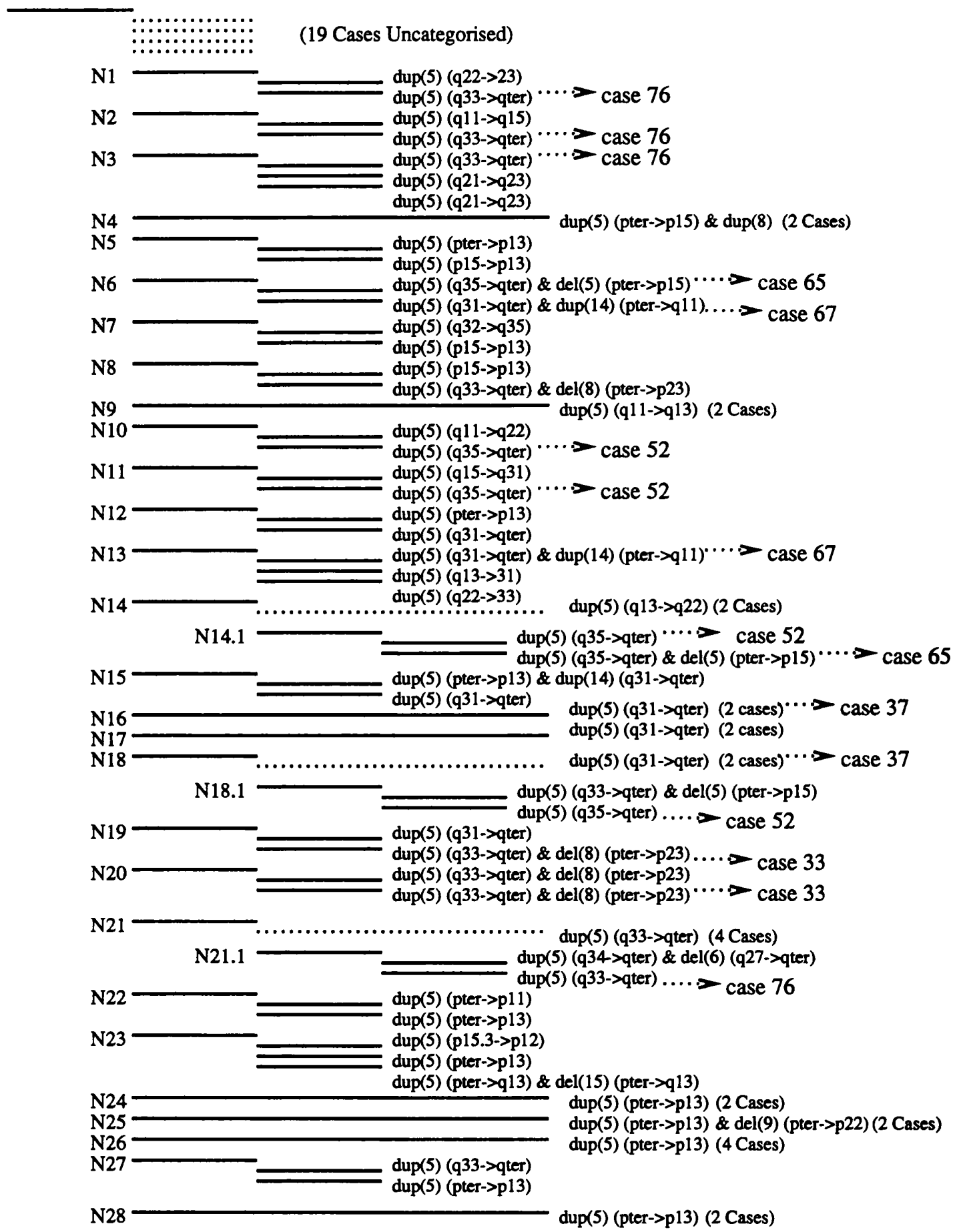

Figure 4.8: Network C5.1: Concept hierarchy generated by cases with a duplication in the number 5 chromosome ( 77 cases). 


\section{$\mathbf{R}$}

(15 Cases Uncategorised)

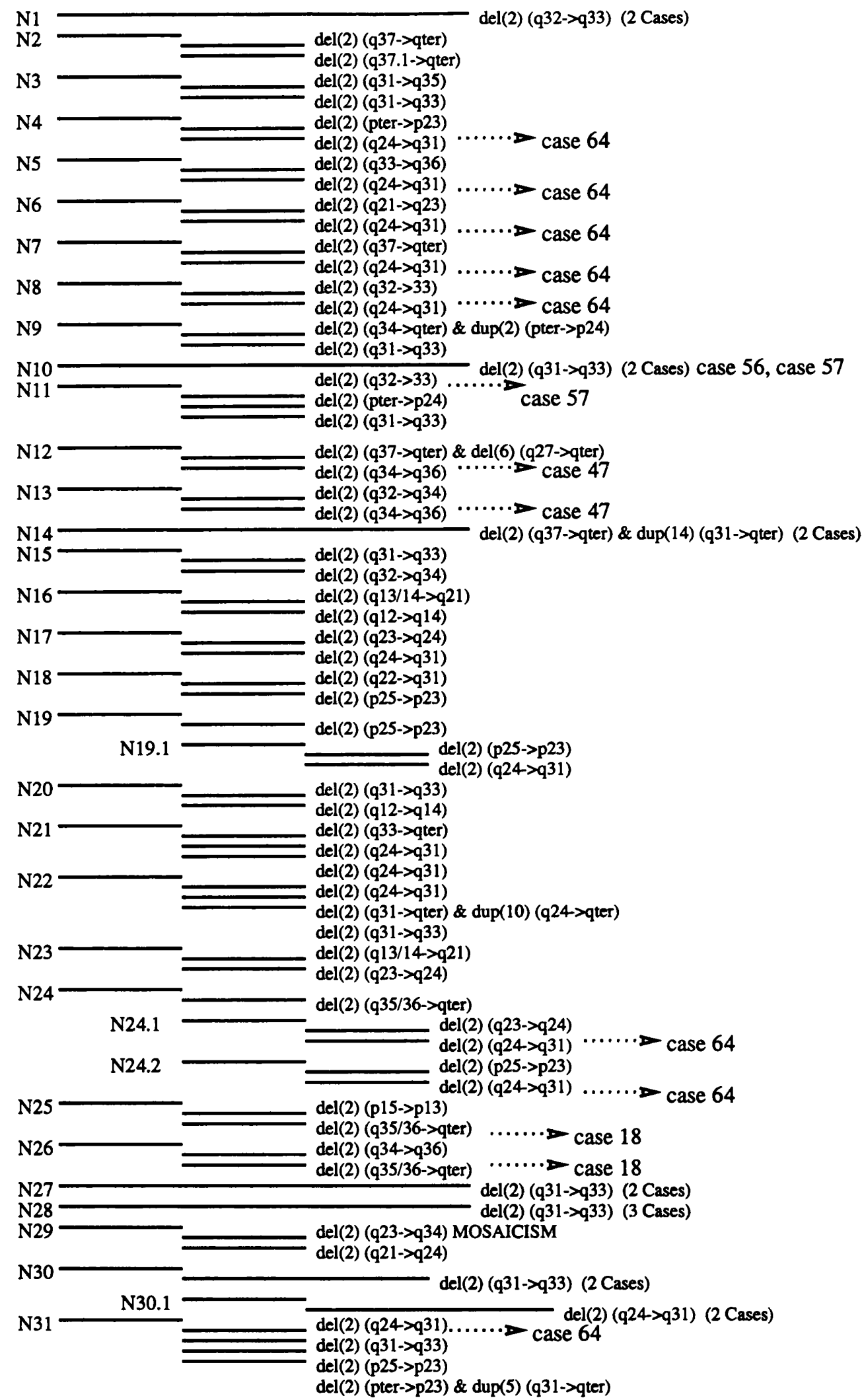

Figure 4.9: Network C2.1: Concept hierarchy generated by cases with a deletion in the number 2 chromosome (72 cases). 


\begin{tabular}{|c|c|c|}
\hline Node N21 Permanent Features & \multicolumn{2}{|c|}{ Stored Case Features } \\
\hline \multirow{4}{*}{$\begin{array}{l}\text { (neurology general mental-retardation) } \\
\text { (abdomen general inguinal-hernia) } \\
\text { (teeth general enamel-abnormalities) } \\
\text { (teeth general dental-caries) } \\
\text { (mouth philtrum simple/sbsent-philtrum) } \\
\text { (mouth philtrum long-philtrum) } \\
\text { (nose nasal-tip bulbous-nasal-tip) } \\
\text { (nose nasal-bridge high/prominent-nasal-bridge) } \\
\text { (nose alae-nasi thick-alac-nad) } \\
\text { (eyes vision strabismus/gaze-paisy) } \\
\text { (forehead small short-forehead) } \\
\text { (stature short short-stature-proportionate) }\end{array}$} & 1 & $\begin{array}{l}\text { (build build-obese truncal-obesity) } \\
\text { (build build-thin low-birth-weight-i-3rd-centile) } \\
\text { (thorax heart ecg-abnormality/conduction-defects) }\end{array}$ \\
\hline & 2 & $\begin{array}{l}\text { (ears external-ears dysplastic-ears) } \\
\text { (thorax heart ecg-abnormality/conduction-defects) } \\
\text { (lower-limbs hip perthes/dysplastic-hip) } \\
\text { (feet general small-feet) } \\
\text { (feet general wide-feet) } \\
\text { (neurology general seizures/abnormal-eeg) } \\
\text { (akin general ecrema) } \\
\text { (build build-thin low-birth-weight-i-3rd-centile) }\end{array}$ \\
\hline & $\mathbf{3}$ & (hands fingers brachydactyly) \\
\hline & 4 & $\begin{array}{l}\text { (build build-thin low-birth-weight-i-3rd-centile) } \\
\text { (cranium general microcephaly) } \\
\text { (upper-limbs elbow limited-movement-elbow) }\end{array}$ \\
\hline
\end{tabular}

Table 4.7: Description of Node N21 in Network C5.1.

Node N21 only comprises permanent features and is shown in Table 4.7, along with the individual case descriptions. In Network C2.1 three nodes list permanent features in their concept descriptions: Nodes N24, N30 and N31. However, none of these nodes demonstrate a clear grouping of cases with the same banding defect. Node N28 does link three cases with the same banding defect ( $\mathbf{3} 31 \rightarrow$ qter), but does not list any permanent features in its concept description.

Referring to Figure 4.7 it can be seen that both these networks have a mean group accuracy factor $(\overline{G A F})$ that is greater than that achieved with the SDR data set (but lower than that of the LDDB data set). The network generated by cases with a duplication in the number 5 chromosome (denoted C5.1 in Figure 4.7) has a $\overline{G A F}$ of +0.596 , whilst the network generated by cases with a deletion in the number 2 chromosome has a $\overline{G A F}$ of 0.0 . A number of factors must be considered when evaluating the performance of UNIMEM with the cases denoted by chromosomal abnormalities. These data sets are all of the same disease family. Thus, when two cases match (have the same banding defect) the contribution to the $G A F$ is +1.0 . If they do not match, then the negative contribution is only -0.25 . A negative contribution of -1.0 does not occur with the chromosomal data sets. However, these data sets are much larger, and case descriptions 
have greater variance. The maximum number of listed features for a case in the dup(5) set is 28 , and the minimum is 2 (the mean is 15.14 ). For the del(2) set the maximum number of features is also 28, the minimum is $\mathbf{5}$ and the average is $\mathbf{1 7 . 4 3}$. Furthermore, these are not prepared data sets. When the non-chromosomal cases for these experiments were being compiled, the physician specifically selected cases that were known to group according to known (or related) syndromes. The two data sets from HCDB consist of all the cases with either dup(5) or del(2) chromosome defects listed in the database. That is to say, there has been no pre-selection of desirable data. As well as having greater variation in case descriptions, these data sets carry greater environmental noise. It was noted in Chapter 1 that chromosome analysis is not perfect, and cases stored in HCDB are labelled according to what a human expert sees through a microscope. The accuracy of the recorded banding defects is not, therefore, considered perfect [88].

\subsection{Discussion}

In order to assess the relative strengths and weaknesses of a CBL model such as UNIMEM, particularly with respect to the designated domain, the first question that needs to be answered is "what the above experiments actually demonstrate?" The application of CBL as described above facilitates two essential facets of a CBR system. Firstly, the network that is generated from the input (the case data set) is essentially an organised case-base, i.e., it is a database of cases and prototypical concept nodes (or generalised cases) that map onto syndrome entities. Secondly, the algorithm that formed the concept hierarchy also constitutes a (case-based) diagnostic reasoner. That is to say, it can be utilised to retrieve similar objects from the case memory. To illustrate this, consider Network A.1 (Figure 4.5), generated from the Acrocephalosyndactyly data set with parameter settings: $E_{t}=5, G_{t}=5$ and $P_{t}=3$, and a new case comprising those features listed in Table 4.8. To process the new case, the top-level function is invoked and search commences at the root node (see Figure 4.10). The program sorts through each child node of the root evaluating with two concepts: N1 and N9. The new case matches Node N1 on five features: craniosynostosis, frontal-bossing, hypertelorism, broad thumbs and broad hallux. The remaining three features are compared with the three instances (or cases) stored at $\mathrm{N} 1$, however, there is insufficient similarity to generalise a new sub-node (as $G_{t}=5$ ). Thus, the new case is stored at $\mathrm{N} 1$, and is constructed with the discriminative features: 


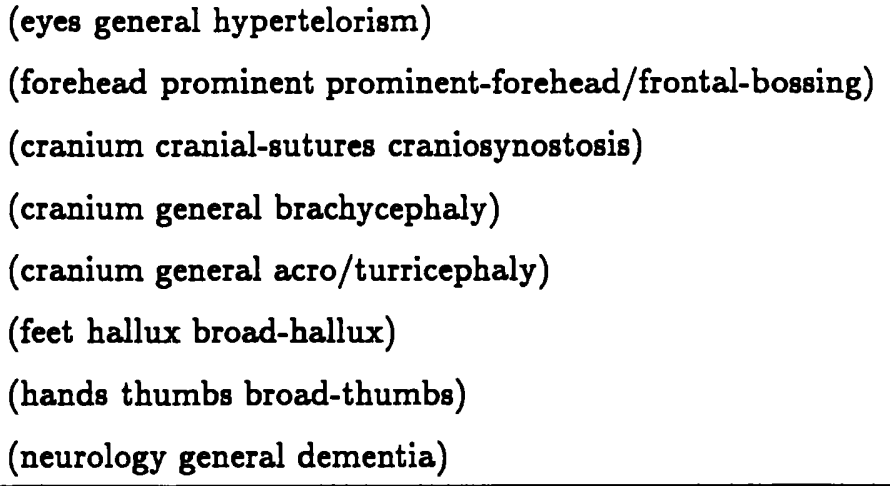

Table 4.8: Hypothetical case with craniosynostosis.

brachycephaly, acro/turricephaly and dementia. Node $\mathrm{N} 1$ is then returned to indicate where in the network the new case has been stored, which implies a classification. As the concept node N1 maps to a theoretical syndrome description, the returned location, or classification, provides a potential diagnosis. Similarly, the new case matches Node N9 on five features: craniosynostosis, acro/turricephaly, brachycephaly, frontal-bossing and hypertelorism. Again this leaves three features of the new case to compare with Node N9.1 and the cases stored at Node N9, which does not prove sufficient for generalisation to take place. Thus, the new case is also stored at Node N9, but in this instance the new case is constructed with the features: broad hallux, broad thumbs and dementia. Following comparison with all the nodes linked to the root, the input case is matched with each of the four unclassified instances (5754, 7312, 8967 and 6566). However, none of these match on enough features with the new case in order to create a new concept node.

The program, therefore, returns two concepts corresponding to two locations (or diagnoses) within memory at which the new case may be stored. Furthermore, this search has been confined by the sorting procedure (unlike LDDB which compares each syndrome record with the index). If the system is to be employed in a case-based reasoning mode only, then the locations, or the actual objects returned, are all that is required to enable a differential diagnosis. However, the algorithm described operates in a case-based learning mode so it actually stores the new case and updates the relevant category information. The revised concept description for nodes $\mathrm{N} 1$ and N9 are shown in Table 4.9. Node N9 has not changed (matching features were already permanent), however, the predictability scores for each of the non-permanent features that matched with the new case on Node 


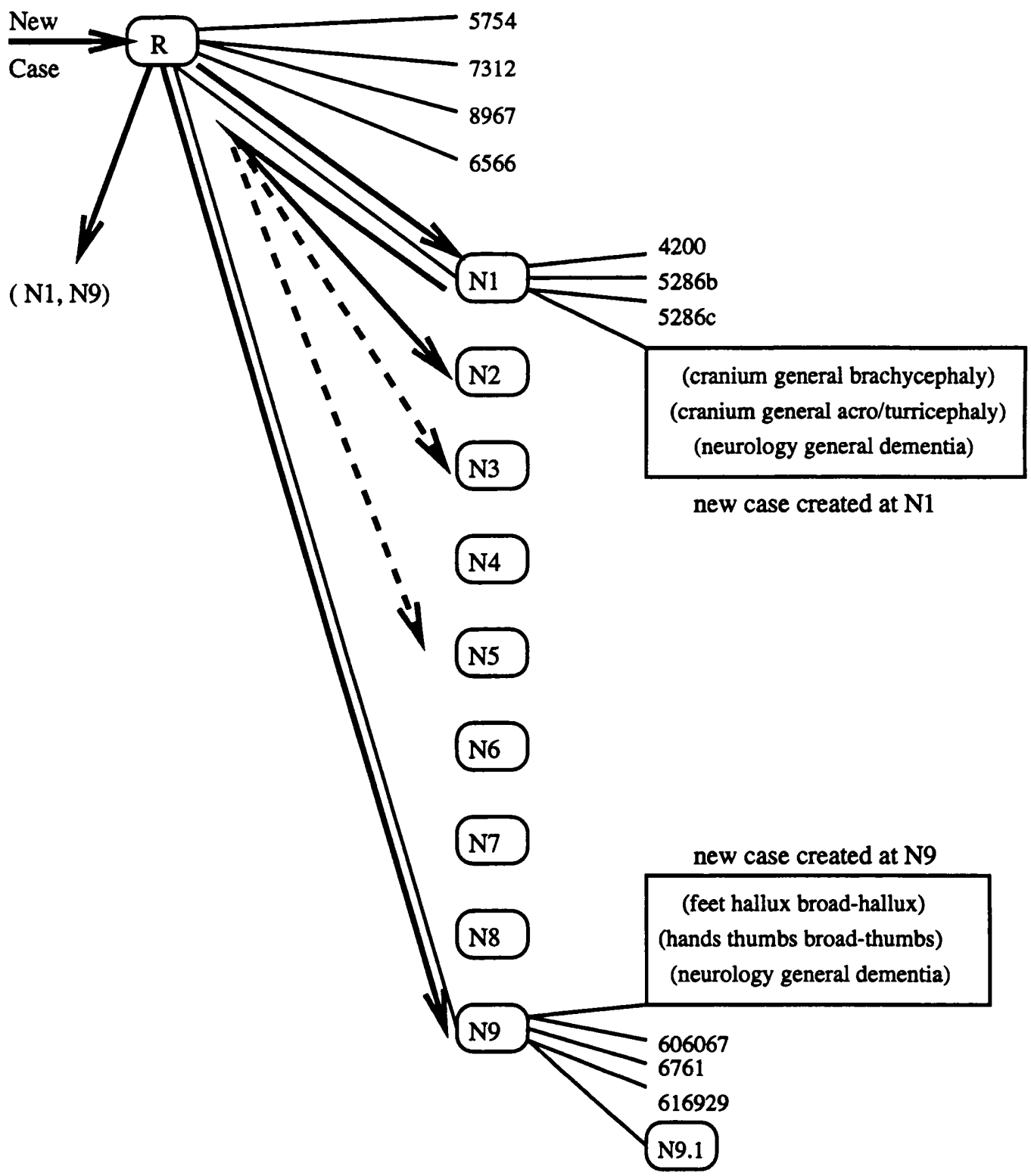

Figure 4.10: Diagnostic search with Network A.1. 


\begin{tabular}{|l|l|l|}
\hline Node & Permanent & Non-permanent \\
\hline N1 & $\begin{array}{l}\text { (feet hallux broad-hallux) } \\
\text { (hands thumbs broad-thumbs) } \\
\text { (cranium cranial-sutures craniosynostosis) } \\
\text { (eyes general hypertelorism) } \\
\text { (forehead prominent frontal-bossing) }\end{array}$ & $\begin{array}{c}\text { (neurology general } \\
\text { normal-intelligence) }\end{array}$ \\
\hline N9 & $\begin{array}{l}\text { (eyes general prominent-eyes-including-proptosis) } \\
\text { (eyes general hypertelorism) } \\
\text { (forehead prominent frontal-bossing) } \\
\text { (cranium cranial-sutures craniosynostosis) } \\
\text { (cranium general brachycephaly) } \\
\text { (cranium general acro/turricephaly) }\end{array}$ & \\
\hline
\end{tabular}

Table 4.9: Revised node descriptions for Network A.1.

N1 have been incremented, causing the predictability threshold $\left(P_{t}=3\right)$ to be reached. Thus, Node N1 has a revised concept description.

To summarise, the CBL model described above provides a basic case-based system that can perform default reasoning (through object retrieval), and can automate learning (through category revision). Furthermore, reasoning involves confined search with an associated similarity assessment, albeit simplistic. It has not been too difficult to map these processes to real world activities in dysmorphology as indicated by Table 2.4 of Chapter 2. However, by mapping the UNIMEM algorithm to the real world, weaknesses generic to this type of CBL model are exposed.

\subsection{A Critique of UNIMEM}

The non-uniformity of case descriptions has already been noted as a key problem within dysmorphology. The above experiments corroborate this fact. It was shown that the general level of detail of case descriptions can be influential in system operation. As separate, consistent data sets were employed (i.e., case descriptions were consistent within each data set), the practical implications were merely to adjust the various system parameters. However, these laboratory conditions would be unlikely in the real world where a great deal of variation in case descriptions could be expected. Furthermore, cases would be analysed by experienced specialists who would be able to distinguish abnormalities of diagnostic significance. The CBL model described so far does not have this capability.

This leads to another aspect of UNIMEM (and models of concept formation in general) which does not map well with dysmorphology. The learning process involved with 
UNIMEM has a goal of establishing its own model of diagnosticity, based on frequency. Those features made permanent on a concept, derived purely from incidence within the stored population, are intended to assign a level of certainty to a node [50]. That is to say, they imply prototypicality within the concept, which can be construed as a type of feature salience. Again, it has already been noted that in dysmorphology the relationship between diagnosticity and frequency is tenuous, and can certainly not be assumed. Furthermore, even though this informal implication of diagnosticity is derived through incidence, the salience of individual features is not involved in any assessment of similarity. This is still performed by a simple count of the overlapping features.

An important aspect of dysmorphology that UNIMEM does exhibit is that an individual case may be linked to more than one category. In a purely reasoning mode, finding more than one location within the case-base promotes a possible differential diagnosis. However, UNIMEM automatically integrates each new case and updates the relevant category information. A result of this is that one individual case may be physically stored at more than one location in memory, and is only comprised of features that do not match the parent node. Also, it may be stored in terms of different features depending on how it matches with each parent node (as the above example demonstrates). These actions are typical of a discrimination algorithm that assumes hierarchical top-down search. However, these mechanisms are not intuitive within dysmorphology. This is demonstrated in the extreme by Case 0003 (see Figure 4.5), which is stored at Node N7 in Network A.1. The actual list of dysmorphic features for Case 0003 comprises a conjunction of the permanent and non-permanent features listed for Node N7 (a total of seven features). Consequently, Case 0003 has actually been created and stored with no specific features (a link is in place to show that the case exists, but there are no further distinguishing features other than those on the node description). In the real world, physicians are not restricted by hierarchical top-down search when looking for a potential diagnosis. Gestalt recognition may prompt an expert to select a specific case directly from memory, without any (conscious) search. Furthermore, in a situation such as this, it is difficult to imagine that a case description would not be retrieved in its entirety. That is to say, it would not be analysed only in terms of those anomalies that are distinctive from the syndrome with which it is associated, and the relevant medical record would document all the dysmorphic features of the case. Also, an individual patient would be documented only once, more than likely by the examining physician. Even if the child showed similarity to more 
than one syndrome category, which would be noted, the malformation pattern would not be recorded at more than one (categorical) location in terms of different set of anomalies.

Another weak aspect of CBL models such as UNIMEM is that the order in which individual cases are presented can affect the structure of the resulting concept network ${ }^{10}$. Referring back to Figure 4.5 and Table 4.6, Node N7 does not have the feature (ears external-ears dysplastic-ears) within its concept description. However, of the four instances stored below Node $\mathrm{N} 7$, three cases do have this feature listed in their description (9605, 0004 and 0002). As the predictability threshold, $P_{t}$, is equal to 3 , this anomaly would be expected on the concept description. However, it is not, and this is due to the order in which the cases were processed. Node N7 was initially created with the overlapping features of Cases 0002 and 0003 . As Case 0003 does not have dysplastic ears this feature was not abstracted to the concept description. In the UNIMEM model, predictability is only incremented on the node description, not on the cases stored below it. Furthermore, once a node description is created, features may only be deleted (if the predictability becomes too low), they cannot be added. In the real world, when a syndrome description (which would have been abstracted from a number of individual cases) is under scrutiny due to new evidence (from a newly presented case) both the syndrome description, and those of the cases already associated with it, would be taken into consideration. That is to say, the revision of the syndrome prototype would not merely involve analysis of the new case and the syndrome description, it would also take into account previous diagnoses. Thus, the ordering of individual cases does not have an impact on syndrome descriptions.

\subsection{Conclusions}

The key process in the operation of UNIMEM is similarity assessment. There are two types of similarity assessment that control UNIMEM's performance: evaluation and generalisation. Evaluation compares a case to a node, and generalisation matches a case with a case. Both types of match are performed by counting the overlapping features between the two entities. UNIMEM does not account for the relative significance, or salience, of individual features with respect to its similarity assessment. An indication

\footnotetext{
${ }^{10}$ Lebowitz points out that ordering can result in similar, but non-identical concept hierarchies, although over time, and with sufficient data the effect is not strong [50].
} 
of prototypicality for individual features, with respect a concept description, is derived from their incidence across the cases that are grouped at the concept node. This does not, however, generate a measure of feature salience for use within future assessments of similarity.

Ultimately, the performance of the UNIMEM algorithm (as with any instance-based learning algorithm) relies on its model of similarity assessment. If the program is to accurately group cases (i.e., achieve correct grouping according to category membership), and form useful concepts (in which category information is sufficient to distinguish a concept and classify relevant cases accordingly) based purely on the frequency of individual features across its training set, then it would be desirable that the training set be sufficiently large and described in a consistent or uniform manner. Unfortunately, the dysmorphology domain does not usually provide this type of environment. Dysmorphology is inherently noisy, incomplete and recorded cases are generally not uniform in their descriptions. Furthermore, it cannot be assumed that the salience, or diagnosticity, of specific abnormalities is directly proportional to their incidence amongst the general population. Thus, the similarity assessment performed by UNIMEM, as it stands, is not adequate to achieve high levels of accuracy with cases of dysmorphic syndromes. If, however, the similarity assessment performed by the UNIMEM program could be extended in order to utilise known diagnostic significance, rather than merely attempting to derive such knowledge through incidence, then perhaps its performance could be improved with respect to cases of dysmorphic syndromes. Fortunately, domain knowledge of this type is available. The following chapter describes an enhanced version of the UNIMEM program that incorporates a domain model of diagnostic significance. This is used to extend the similarity functions of the algorithm, and ultimately improve on its performance with respect to the data sets of chromosomal and non-chromosomal syndrome cases. Consequently, the extended UNIMEM model improves on its original design. Whilst these experiments are dedicated to the field of dysmorphic syndromes, the enhanced version of the program provides a fairly generic improvement on the original version. A strength of the UNIMEM program is that its functionality is very modular, so that an extended similarity model can be incorporated without altering the main algorithm. Also, the diagnostic model (which has been acquired from the workers of LDDB) provides an independent body of knowledge. The revised similarity functions utilise this domain dependent knowledge to perform evaluation and generalisation, however, provided that a 
relevant domain model is available, then the extended version of the program could be used within any applicable field.

\subsection{Summary}

- The UNIMEM algorithm can be linked to both case-based reasoning and incremental concept formation. Certain facets of the algorithm are desirable with respect to the dysmorphology domain. Firstly, it generates and operates with graph structures and so can classify an individual case beneath more than one category. Also, its basic matching operator is polymorphic, i.e., the similarity between two entities need not be exact in order for matching to succeed.

- The UNIMEM algorithm provides a model of case-based reasoning and learning. Its operation implicitly works in a classification case-based reasoning mode by searching the case-base for the best location at which to store a new case, whilst effectively establishing which cases in the case-base (including generalised cases, or prototypes) are most similar to the input. Furthermore, it automatically reorganises the category structure of the case-base each time a new case is integrated within it. Thus, UNIMEM performs automated case-based learning.

- The performance of UNIMEM can depend on a number of factors including the accuracy and level of detail of case descriptions. Added to this, the operation of the program can be empirically guided through the selection of parameter values.

- The UNIMEM model does not map perfectly with some aspects of the dysmorphology domain. For instance, the prototypicality of features on a concept is directly proportional to frequency, cases are only stored in terms of their distinguishing features (and can actually be stored more than once with different descriptions), and a global hierarchical category structure is assumed.

- The similarity assessment performed by the UNIMEM program is limited. Matching is performed merely in terms of overlapping feature counts, and does not involve individual feature salience. The performance of UNIMEM, with respect to data from the dysmorphology domain, could be improved by extending its model of similarity assessment to include the diagnostic significance of individual abnor- 
malities. A diagnostic model has been made available (from LDDB) and provides the platform for an enhanced version of the program. 


\section{Chapter 5}

\section{Integrating Domain Knowledge within the CBL Model}

\subsection{Introduction}

It was demonstrated in the previous chapter that the UNIMEM algorithm calculates the predictability of individual features within a node description according to the incidence of the features across the set of cases stored at the node. Once a specified frequency is achieved, a feature is made permanent on the node, implying that the feature is predictable for that concept. The strength of a concept is thus implied by the existence of permanent features in its description. Thus, the permanency of a feature implies its salience with regard to the relevant concept, which can in turn be construed as an indication of diagnostic strength. Permanent features constitute strong evidence for a match. However, no additional weight is assigned to permanent features during the matching process. Permanent features are merely fixed to the relevant concept. Their predictability score does not alter and there is no specific indication of individual feature salience. There is a mapping here with the syndrome descriptions stored in LDDB. The dysmorphic features listed for a syndrome do not have an indication of their individual diagnostic significance with respect to the syndrome. This is deliberate because syndromes can be based on a small number of individual cases. Perhaps over time sufficient information will become available to allow diagnostic indicators within syndrome descriptions. However, the UNIMEM model would still not create a scale of diagnosticity across different node features even if many cases were available. Therefore, whilst UNIMEM does imply the 
strength of a concept it does not specifically generate a model of diagnostic significance.

Referring once more to the dysmorphology domain, in which diagnostic significance clearly plays an important role in similarity assessment, the UNIMEM algorithm, as it stands, has an obvious weakness. It does not distinguish between hard, soft and occasional abnormalities during evaluation and generalisation. Rather the algorithm relies purely on parameter settings (which relate to frequencies), or counts, a weakness clearly demonstrated when UNIMEM is applied to the SDR data set (described in Chapter 4). This chapter analyses an enhanced version of the UNIMEM algorithm which incorporates a domain model of diagnostic significance, and which demonstrates an improved performance with respect to the experimental data comprising non-chromosomal and chromosomal disorders.

\subsection{The LDDB Model of Diagnostic Significance}

The earlier description of LDDB noted that it has a master list of over 1200 dysmorphic features grouped by clinical regions. It was also stated that no indication of diagnosticity is associated with individual abnormalities, and that the selection of a useful boolean combination of features (in order to form an index to the database) is a function left to an experienced physician. There has been some work, however, dedicated to the assignment of 'diagnostic indicators' to the master list. These are pointers to the salience of each specific anomaly in the master list and correspond to its relative diagnostic strength across all known syndromes. This has been done in order to increase the scope of LDDB so that less experienced physicians will be able to refer to the diagnostic pointers when selecting an index. Ultimately this will assist in establishing a differential diagnosis. These diagnostic assignments have been compiled for future versions of LDDB.

The representation of feature significance in LDDB is straightforward. Each feature is assigned an integer value ranging from 1 to 4 . These integer values represent a diagnostic scale of significance. It is important to note that the actual values (i.e., 1 to 4 ) do not correspond to relative diagnostic strength. The integer values are merely a convenient representation for a scale comprising four levels (for instance, an alternative representation comprising the alphabetical characters $a, b, c, d$ could have been used). Features with an associated scale value of 4 correspond to hard anomalies that constitute good indexes in LDDB. Features with an associated scale value of 3 are secondary features 
that, alone, would not make a good index. However, one or two of these features combined with a hard anomaly may form a good index. Alternatively, a sufficiently large group of secondary features may constitute a good index. Features with a an associated scale value of 1 or 2 do not make good indexes in LDDB (these correspond to common or occasional features ${ }^{1}$ ).

The assignment of these four diagnostic levels is not a perfect representation of diagnostic significance. The tenuous correlation between incidence and diagnostic importance still exists. In LDDB's mode of operation, the ranking of features is designed with an emphasis on selecting good indexes rather than a specific coding of diagnostic power for a general assessment of similarity. Thus, the four levels of significance reflect indexing power rather than diagnostic weight (e.g., mental retardation has an associated value of 2). Generally, however, hard features (i.e., those with a scale value of 4 ) do constitute anomalies of high significance, and whilst not ideal, the assigned levels do provide a readily accessible and simple form of domain knowledge.

\subsection{The W-UNIMEM Algorithm}

With the availability of a diagnostic model, the UNIMEM functions that perform evaluation and generalisation can be modified in order to take into account the diagnostic significance of individual abnormalities rather than simply counting the number of matching features. Evaluation of whether or not a case sufficiently matches a node in order for search to propagate to the relevant sub-nodes is controlled by the parameter $E_{t}$. This count is assessed by a function that decides whether or not there are too few matching features for the evaluate function to be invoked (see Figures 4.2 and 4.3 in Chapter 4). When a new case enters the system, it will first be compared with all the top level concept nodes, i.e., the child nodes of the root, followed by a comparison with unclassified instances. In terms of dysmorphology nomenclature, these top level conceptual categories refer to either syndromes or syndrome families that are distinguished by a pattern of principal features. A successful evaluation, or a positive match between a new case and a concept node, should be one in which the new case exhibits a satisfactory number of these principal abnormalities. With respect to the domain

\footnotetext{
${ }^{1}$ This representation is not unlike that of GENDIAG in which three levels are defined. However, in GENDIAG only features of the highest level are employed as indexes.
} 
model of LDDB, principal features are those that have an associated scale value of 3 or 4. Thus, the evaluation of whether a new case matches a node in the network can be based on those features that match with an associated score of 3 or more, rather than a general count of all matching features. For example, consider Node N7 of Network A.1 shown by Figure 4.5 and described in Table 4.6 of Chapter 4. According to the domain model, two of the listed anomalies carry the highest diagnostic weight denoted by the scale value 4: hands fingers absent-fingers-or-oligodactyly and upper-limbs forearm hypoplastic-or-absent-ulna. It is feasible that a case could match Node N7 on both these features yet fail to evaluate with the concept if the evaluation threshold, $E_{t}$, is not reached. However, in the real world, any syndrome that matched a patient on these two anomalies would certainly be considered, at least in the initial stages of the investigation. Thus, with the CBL model such an evaluation should really succeed. This is achieved by modifying the evaluate function to take into account the associated diagnostic weight of individual features rather than merely counting the overlap between the case and the node.

The modified evaluation condition is defined by equation 5.1. Two new parameters are introduced: $W_{4}$, an integer value corresponding to the weight assigned to a feature listed within the set of hard abnormalities (denoted by $S_{4}$, and which corresponds to those features with an associated scale value of 4 ), and $W_{3}$, which is the equivalent weight assigned to secondary features (where $S_{3}$ is the set of secondary features, i.e., with a significance value of 3$)^{2}$. The condition defined by equation 5.1 requires that the combined associated weights assigned to matching features with scale values of 3 or 4 is greater or equal to the evaluation threshold $E_{t}$. Thus, the actual weights that are used in this calculation are those assigned to $W_{4}$ and $W_{3}$.

$$
\sum_{i \in H} W(i) \geq E_{t} \quad W(i)= \begin{cases}W_{4} & \text { if } i \in S_{4} \\ W_{3} & \text { if } i \in S_{3} \\ 0 & \text { otherwise }\end{cases}
$$

Referring to the previous example of Node N7 in Network A.1, which lists two features from the set of hard anomalies $S_{4}$, even if these two features provide the only overlap with a case description, in order to be consistent with real-world procedures, these features

\footnotetext{
${ }^{2}$ The aignificance between hard features and secondary features is far greater than the corresponding scale values 4 and 3 . It is important to note that these values are not themselves meant to suggest comparative diagnostic weight.
} 
should provide sufficient diagnostic weight for evaluation to succeed. Thus, a general guideline for the (empirical) selection of diagnostic weights, and the relevant threshold level (which in this case is $E_{t}$ ) can be inferred from this example. That is to say, the value of $W_{4}$ should be sufficient in relation to $E_{t}$ such that two hard abnormalities (or one hard anomaly plus one or two secondary dysmorphic features) provide enough diagnostic significance for evaluation to succeed. The following section provides further discussion concerning the setting of system parameters, including $W_{4}$ and $W_{3}$.

If the input case evaluates successfully with a node $N$, then it will be evaluated against the sub-nodes, or children, of $\mathrm{N}$. The top level function is recursively called with respect to each child node that is indexed by a feature exhibited by the input case that has not already been matched with the parent. In terms of dysmorphology, this process is comparable to an investigation of the similarity between a new patient and the variations of a syndrome family (once overall similarity with the syndrome group has been established) in order to arrive at a more specific diagnosis. As with the general similarity assessment between a patient and a syndrome family, which is based primarily on a pattern of principal abnormalities, the isolation of one specific disorder amongst related syndromes would also be a result of at least one significant distinguishing anomaly. It is difficult to define 'distinguishing significance' in terms of the numeric values that are encoded within the master list of dysmorphic features. These levels have been compiled in a context-free manner. That is to say, they represent a general assessment of usefulness in terms of indexes. When an investigation is focussed on a particular differential diagnosis, the significance of individual features would be much more apparent to an expert who could make an informed decision based on all the available information. The domain knowledge available in this instance only partly models that which is available to physicians. The practical conclusion of this is that, although in the context of an actual examination secondary features (belonging to the set $S_{3}$ ) may be discriminative, in terms of the algorithmic process of the modified CBL program, and in particular the functionality that decides whether or not sub-nodes are indexed, only hard features (i.e., features in the set $S_{4}$ with an associated weight $W_{4}$ ) will be considered.

In very much the same vein as the modifications to the evaluate function, the generalise function shown in Figure 4.3 can also be adapted to account for levels of feature significance. A new concept node is generated if the set of matching features $(\mathrm{H})$ is large enough (as defined by the generalisation threshold, $G_{t}$ ). As with the evaluate 
function, the generalise function has been modified to operate on the combined weight of hard and secondary features that match. The revised condition for generalisation is shown by Equation 5.2. Two new parameters account for the weight assigned to primary and secondary anomalies when generalisation is performed: $Y_{4}$ and $Y_{3}$ respectively. In the modified program, for generalisation to succeed, i.e., for sufficient similarity to exist in order to create a new sub-node, the condition defined by equation 5.2 must be satisfied.

$$
\sum_{i \in H} Y(i) \geq G_{t} \quad Y(i)= \begin{cases}Y_{4} & \text { if } i \in S_{4} \\ Y_{3} & \text { if } i \in S_{3} \\ 0 & \text { otherwise }\end{cases}
$$

It is important to note that the weights assigned for evaluation: $W_{4}$ and $W_{3}$, are not the same as those for generalisation: $Y_{4}$ and $Y_{3}$. The principle of operation is the same, however. The combined sum of the weights assigned to hard and secondary anomalies shared by both cases should provide sufficient evidence, or significance, to suggest a more general node (which is abstracted from the overlap between the two cases). However, it should be remembered that evaluation and generalisation correspond to two different types of similarity assessment in dysmorphology, and the associated significance of anomalies may vary accordingly. The two conditions defined in equations 5.1 and $\mathbf{5 . 2}$ provide the user with a degree of flexibility in selecting parameter values, such that the conditions for successful evaluation and generalisation can be varied.

The modified UNIMEM algorithm, called W-UNIMEM (or Weighted-UNIMEM) is shown in Figure 5.1. The main algorithm has been modified at two control points. A new function, Evaluation-Weight, which incorporates the condition shown by equation 5.1, is now used to establish if an evaluation should succeed. Also, when child nodes are indexed by discriminating features $(K)$, these features must be hard anomalies as defined by the significance set $S_{4}$. The Generalise function has one modification. It now uses a new function, Generalisation-Weight, which incorporates the condition shown by equation 5.2, to assess if sufficient diagnostic significance between two cases exists in order to create a new node.

It should be noted that the domain knowledge available for experimentation (i.e., feature weights) has been compiled for use with LDDB and is therefore compatible with HCDB (which uses the same master list of dysmorphic features). As described earlier, SDR utilises a case representation based on radiological components (i.e., skull, spine 
W-Unimem(N, I, F)

Let $\mathbf{G}$ be the set of features stored on $\mathbf{N}$.

Let $\mathrm{H}=\left\{h_{i} \ldots h_{n}\right\}$ be the features in $\mathrm{F}$ that match features in $\mathrm{G}$ where $h_{i} \ldots h_{n} \in S_{3} \vee S_{4}$.

Let $\mathrm{K}$ be the features in $\mathrm{F}$ that do not match features in $\mathrm{G}$.

If $\mathrm{N}$ is not the root node,

Then If Evaluation-Weight(H) is too low, or Evaluate(N, H, K) returns TRUE,

Then return the empty list.

Let $\mathrm{S}$ be the empty list.

For each child $\mathrm{C}$ of node $\mathrm{N}$,

If $\mathrm{C}$ is indexed by a feature $k$ in $\mathrm{K}$ and $k \in S_{4}$,

Then let $S$ be Union(S, Unimem(C, I, K)).

If $S$ is the empty list,

Then for each instance $J$ at node $N$,

Let $\mathrm{S}$ be Union(S, Generalise(N, J, I, F)).

If $S$ is the empty list,

Then store I as an instance of node $\mathrm{N}$ with features $\mathrm{K}$.

Return N.

Generalise(N, J, I, F)

Let $\mathbf{G}$ be the features in instance $J$.

Let $\mathrm{B}=\left\{h_{i} \ldots h_{n}\right\}$ be the features in F that match features in G where $h_{i} \ldots h_{n} \in S_{3} \vee S_{4}$.

Let $\mathrm{K}$ be the features in $\mathrm{F}$ that do not match features in $\mathrm{G}$.

If Generalisation-Weight $(H)$ is high enough,

Then create a new child $\mathrm{C}$ of node $\mathrm{N}$ with features $\mathrm{H}$.

Remove $\mathrm{J}$ as an instance of $\mathrm{N}$.

Let G' be the features in $G$ that are not in $\mathrm{H}$.

Let $F^{\prime}$ be the features in $F$ that are not in $H$.

Store $\mathrm{J}$ as an instance of $\mathrm{C}$ with features $\mathrm{G}$ '.

Store $\mathrm{I}$ as an instance of $\mathbf{C}$ with features $\mathrm{F}^{\prime}$.

Return C.

Figure 5.1: Modified (weighted) UNIMEM algorithm. 
and pelvis). A mapping has therefore been made between anomalies listed in LDDB and the corresponding features in SDR, in order to assign the relevant weights.

\subsection{Results}

The experiments reported in Chapter 4 were repeated using the W-UNIMEM algorithm. The resulting concept networks are shown in Figures 5.2, 5.3, 5.4 and 5.5. Tables 5.1 and 5.2 list the parameter settings that were used to generate the networks in Figures 5.2 and 5.3 respectively. The networks shown in Figures 5.4 and 5.5 were also generated with the parameter settings listed in Table 5.1.

As mentioned in the previous chapter, the operation of UNIMEM is effectively supervised through empirical manipulation of system parameters. The fact that the data sets comprise known diagnoses allows such guidance with respect to establishing suitable parameter scores. The modified program, W-UNIMEM, has additional parameters to those utilised with the original version of UNIMEM. Along with the three threshold parameters $\left(E_{t}, G_{t}\right.$ and $\left.P_{t}\right)$, W-UNIMEM has four weighting parameters: two for evaluation $\left(W_{3}\right.$ and $\left.W_{4}\right)$, and two for generalisation $\left(Y_{3}\right.$ and $\left.Y_{4}\right)$. These correspond to the weights assigned to dysmorphic features that are defined either as hard abnormalities (i.e, $\in S_{4}$ ) or features of secondary significance $\left(\in S_{3}\right)$, as defined by the LDDB diagnostic model. This design facilitates the assignment of different weights according to whether the similarity assessment is evaluation or generalisation, and thus, increases the scope of the empirical guidance that can be achieved. The selection of these scores has not been solely derived through repetitive empirical assessment, however. The previous section discussed a sensible guideline for the relative values assigned to $E_{t}$ and $W_{4}$, such that the significance of a hard anomaly in the real-world be accounted for by the evaluate function. Similarly, the guidelines put forward by Winter and Baraitser [89] have had some influence on the setting of parameters. In selecting an index for LDDB, Winter and Baraitser recommend one hard anomaly plus one or two lower order (or more general) abnormalities (see Chapter 2). This general rule has guided the selection of parameter settings for evaluation, i.e., $E_{t}, W_{4}$ and $W_{3}$. Table 5.1 shows that for the LDDB and HCDB data sets the evaluation threshold $\left(E_{t}\right)$ is set to 7 , the weight assigned to hard abnormalities $\left(W_{4}\right)$ is set to 5 , and the weight assigned to secondary features $\left(W_{3}\right)$ is set to 1 . Thus, if the input case and a node match on one hard anomaly plus two or more 
secondary features (or two hard anomalies) then evaluation will succeed. The equivalent parameter settings for the SDR data set, shown in Table 5.2, reflect the difference in the detail (and size) of the skeletal dysplasias case descriptions. Empirical judgement has shown that if a case and node match merely on one hard abnormality, then this is sufficient for evaluation to succeed in order to significantly improve performance over the original version of UNIMEM. Alternatively, with $E_{t}$ set to $5, W_{4}$ also set to 5 , and $W_{3}$ set to equal 1, if the input case and node match on five secondary features then evaluation succeeds (overall, the SDR cases are described in terms of fewer hard abnormalities and more secondary abnormalities than those of LDDB and HCDB).

\begin{tabular}{|l|c|}
\hline Parameter & Score \\
\hline$E_{t}$ & 7 \\
\hline$W_{4}$ & 5 \\
\hline$W_{3}$ & 1 \\
\hline$G_{t}$ & 4 \\
\hline$Y_{4}$ & 4 \\
\hline$Y_{3}$ & 1 \\
\hline$P_{t}$ & 3 \\
\hline
\end{tabular}

Table 5.1: Parameter settings for Acrocephalosyndactyly, $\operatorname{Dup}(5)$ and $\operatorname{Del}(2)$ data sets.

\begin{tabular}{|l|c|}
\hline Parameter & Score \\
\hline$E_{t}$ & 5 \\
\hline$W_{4}$ & 5 \\
\hline$W_{3}$ & 1 \\
\hline$G_{t}$ & 6 \\
\hline$Y_{4}$ & 6 \\
\hline$Y_{3}$ & 1 \\
\hline$P_{t}$ & 3 \\
\hline
\end{tabular}

Table 5.2: Parameter settings for skeletal dysplasias data set.

The emphasis on how the relevant generalisation parameters $\left(G_{t}, Y_{4}\right.$ and $\left.Y_{3}\right)$ have been set is slightly different from the equivalent evaluation parameters. Evaluation, in essence, refers to a diagnosis performance task. The input case is compared to a 
(prototype) concept, which in turn represents a syndrome, i.e., a possible diagnosis. In this operation matching is generally based on a pattern of features rather than a single anomaly. Generalisation, on the other hand, represents a learning process in which similarity assessment is performed between a subject and a referent that are both cases. The subsequent matching process does not have the constraints of evaluation (or diagnosis), and links are more readily established between cases in terms of a single (although significant) abnormality. This theme is reflected in the relevant parameter settings for all data sets. Both Table 5.1 and 5.2 show that the generalisation threshold is equal to the weight assigned to hard abnormalities. Thus, generalisation will succeed if the input case matches another case on one hard anomaly. For the LDDB and HCDB data sets, the generalisation threshold, $G_{t}$, is set to the value $4, Y_{4}$ is also set to 4 , and $Y_{3}$ is set to 1 . For the $\operatorname{SDR}$ data set the respective values are set such that $G_{t}=6$, $Y_{4}=6$, and $Y_{3}=1$. Again, the difference between the SDR settings and those for LDDB and HCDB cases reflects the variation in case descriptions overall. This aspect is particularly relevant to the relationship between $G_{t}$ and $Y_{3}$. The relative values for these parameters decide on how many secondary features alone (when no hard abnormality matches) would be sufficient for generalisation to take place. For the SDR cases, which are described in terms of a greater number of secondary and occasional features than both the LDDB and HCDB cases, this contextual factor has been taken into account with respect to the relevant parameter values. Thus, it should be noted that empirical judgements have been made in the light of general (real-world) procedures in selecting the parameter values shown by Tables 5.1 and 5.2. Empirical guidance has been achievable, in this instance, because the diagnoses of each case in each data set is known. This is an important facet of these experiments and is discussed further in section 5.5.

Comparing Network A.2, generated by W-UNIMEM (see Figure 5.2), to Network A.1, generated from the original UNIMEM algorithm (Figure 4.5), one can interpret a slight improvement in performance. Only two cases now remain unclassified: Case 8967 (diagnosed as Treacher-Collins) and Case 6351 (also Treacher-Collins), which was formerly grouped with a case of Nager syndrome. Previously Case 5754 (Nager) was unclassified, but now it is grouped with the four cases of Acrofacial Dysostosis with post-axial defects (or Millar syndrome). It was noted previously that this group is characterised by both facial dysostosis in combination with a limb defect. Case 5754 has both facial abnormalities and the matching limb defect (upper-limbs forearm hypoplastic-or-absent-ulna). 
In the first instance, evaluation was based on a count of overlapping features $\left(E_{t}>5\right)$ and failed. In the modified program, however, the aforenamed anomaly carries the highest possible significance $\left(\in S_{4}\right)$. When combined with the secondary features that match, there is now sufficient weight for the evaluation threshold to be reached, and Case $\mathbf{5 7 5 4}$ is subsequently linked to node N7. The slight increase in performance is corroborated by Figure 5.6, which shows the relative mean group accuracy factors for the networks generated by UNIMEM and the revised versions generated by W-UNIMEM. The value of the $\overline{G A F}$ for Network A.2 is +1.52 as compared with +1.38 for Network A.1.

Another variation that can be seen between Network A.1 and the revised Network, A.2, is that the two cases formerly linked together by Node N2, Cases 5286a (Pfeiffer) and 7877 (Saethre-Chotzen), are not joined in Network A.2. Case 5286a has joined three cases with Pfeiffer syndrome (Node N3), and Case 7877 has joined two cases with SaethreChotzen syndrome and one with Apert (Node N4). Cases of Apert and Saethre-Chotzen syndromes still demonstrate a general grouping pattern as in Network A.1 (Nodes N1, N4, N8 and N9). However, whilst Node N4 suggests a more distinctive group of SaethreChotzen cases that did not exist in Network A.1, concepts N9 and N9.1, which formerly spanned 5 cases of Apert and one case of Pfeiffer (thus, suggesting a fairly distinctive grouping of Apert cases) now appear slightly weakened in that less cases of the same diagnoses have been linked.

The revised network (Network S.2) generated by the skeletal dysplasias cases shown in Figure 5.3 demonstrates a marked improvement in performance by the W-UNIMEM algorithm over UNIMEM. The concept nodes of Network S.2 show a much more correct grouping of cases with respect to the known diagnoses. For example, Node N5 groups together all three cases of SEDC (091-0084, 091-0185 and 092-0181), whilst its sub-node (N5.1) links the three cases of SEMD (092-0184, 091-0162 and 091-0096) with one case of Achondrogenesis II (091-0215), which, as is shown in Table 4.4, is a related syndrome. Correct family grouping is also apparent in Node N4, which links the three cases of Asphyxiating Thoracic Dystrophy (two of which were formerly unclassified) with one case of Short Rib Polydactyly Type II (090-0225), whilst its child node (N4.1) links a further four cases of Short Rib Polydactyly. The correct family grouping achieved by the W-UNIMEM algorithm was not demonstrated by Network S.1, and the comparative mean group accuracy factors shown in Figure 5.6 reflect this. The $\overline{G A F}$ for Network S.1 was -0.125 . The revised program achieves a $\overline{G A F}$ of +1.025 . 


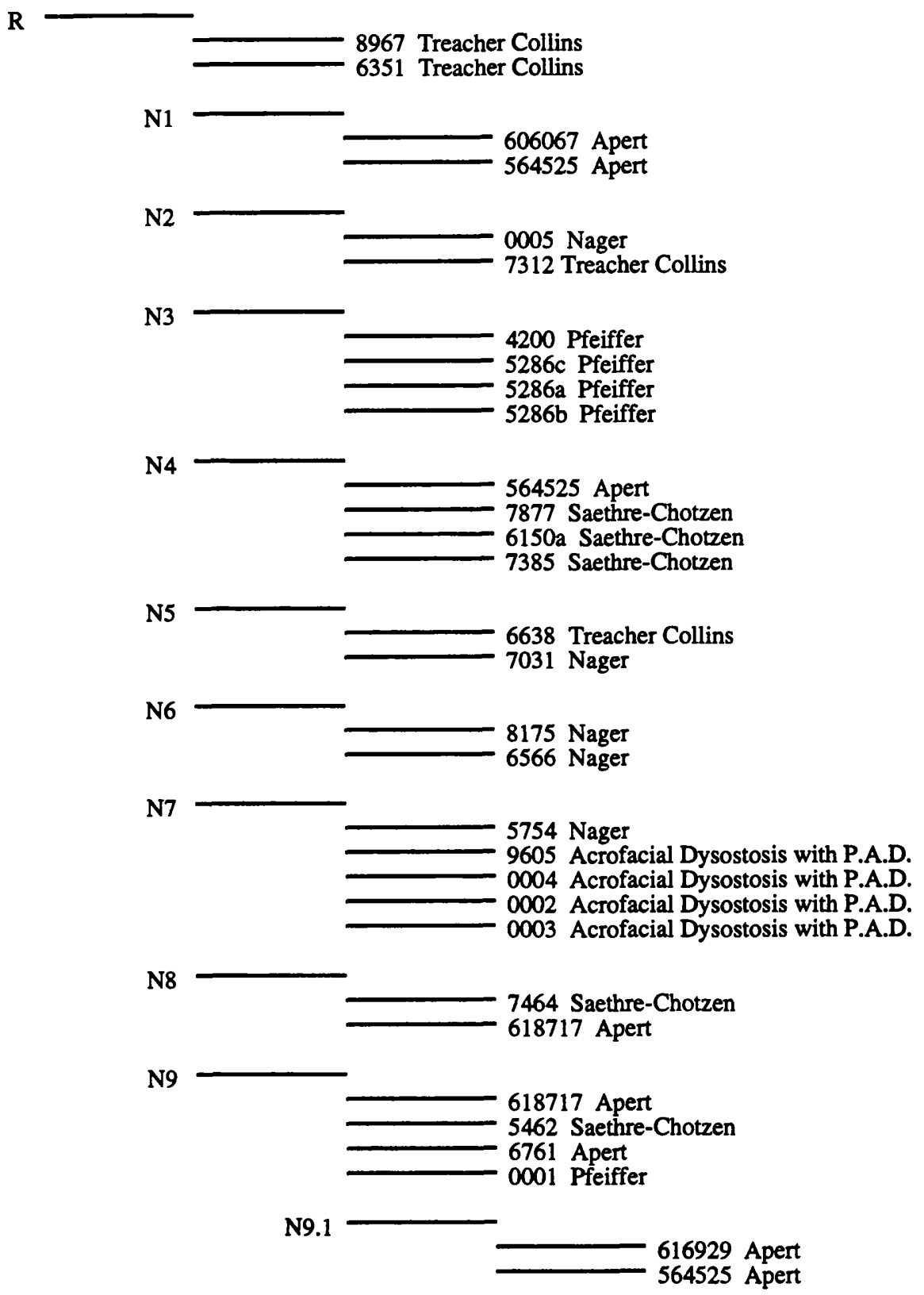

Figure 5.2: Network A.2: Revised concept hierarchy generated by the Acrocephalosyndactyly data set. 
R

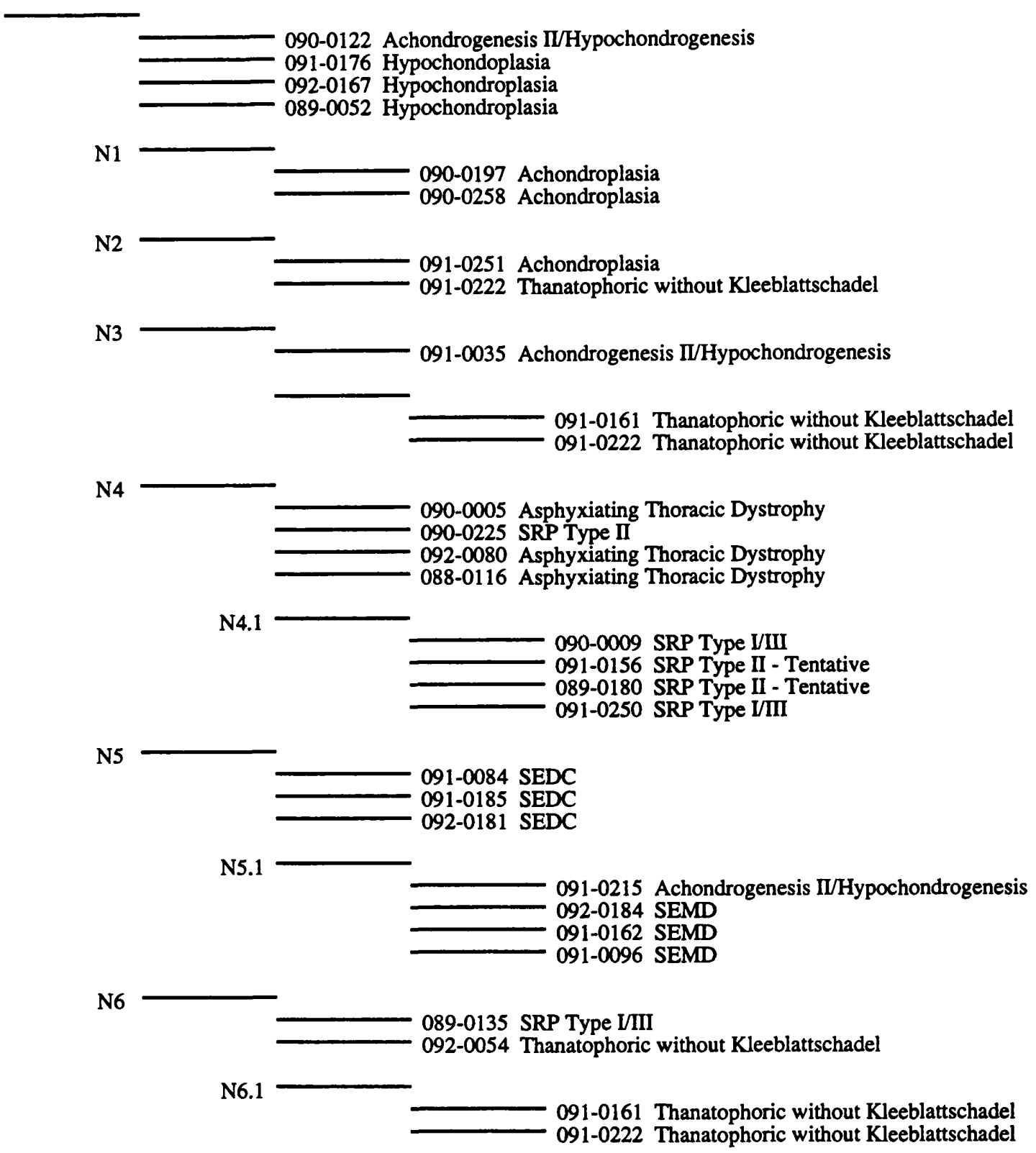

Figure 5.3: Network S.2: Revised concept hierarchy generated by the skeletal dysplasias data set. 


\section{$\mathbf{R}$}

(37 Cases Uncategorised)

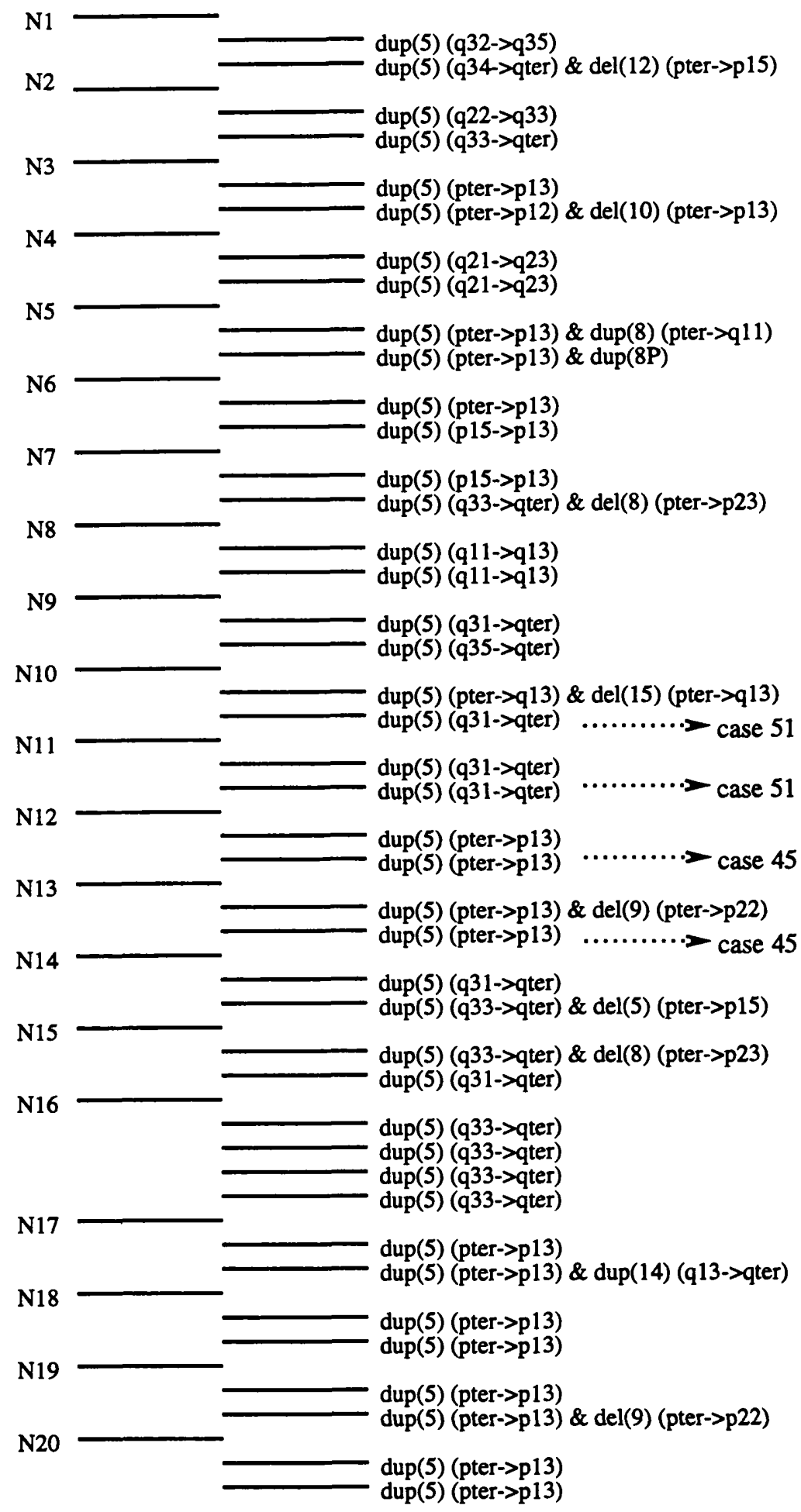

Figure 5.4: Network C5.2: Revised concept hierarchy generated by cases with a duplication in the number 5 chromosome. 
$\mathbf{R}$

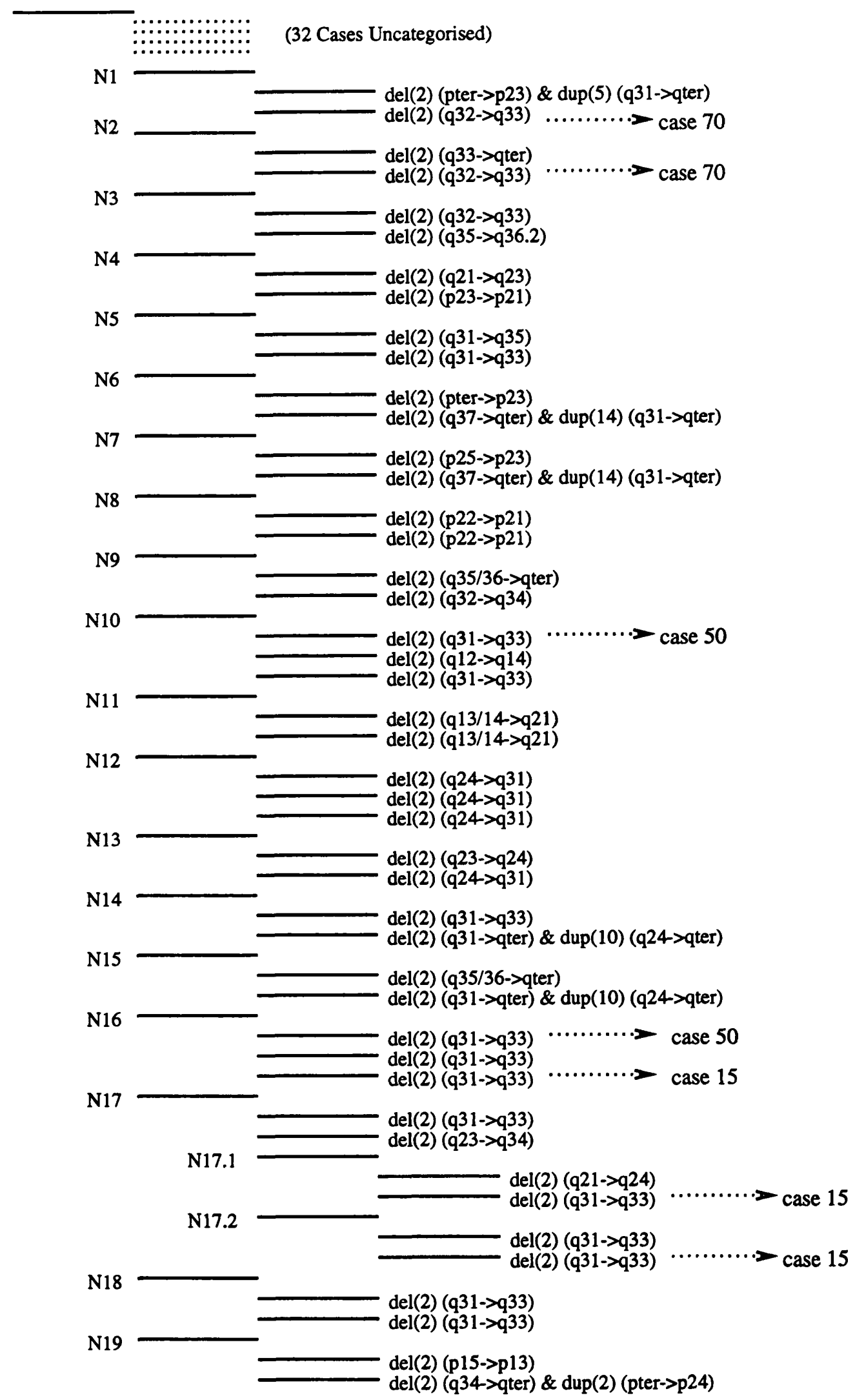

Figure 5.5: Network C2.2: Revised concept hierarchy generated by cases with a deletion in the number 2 chromosome. 


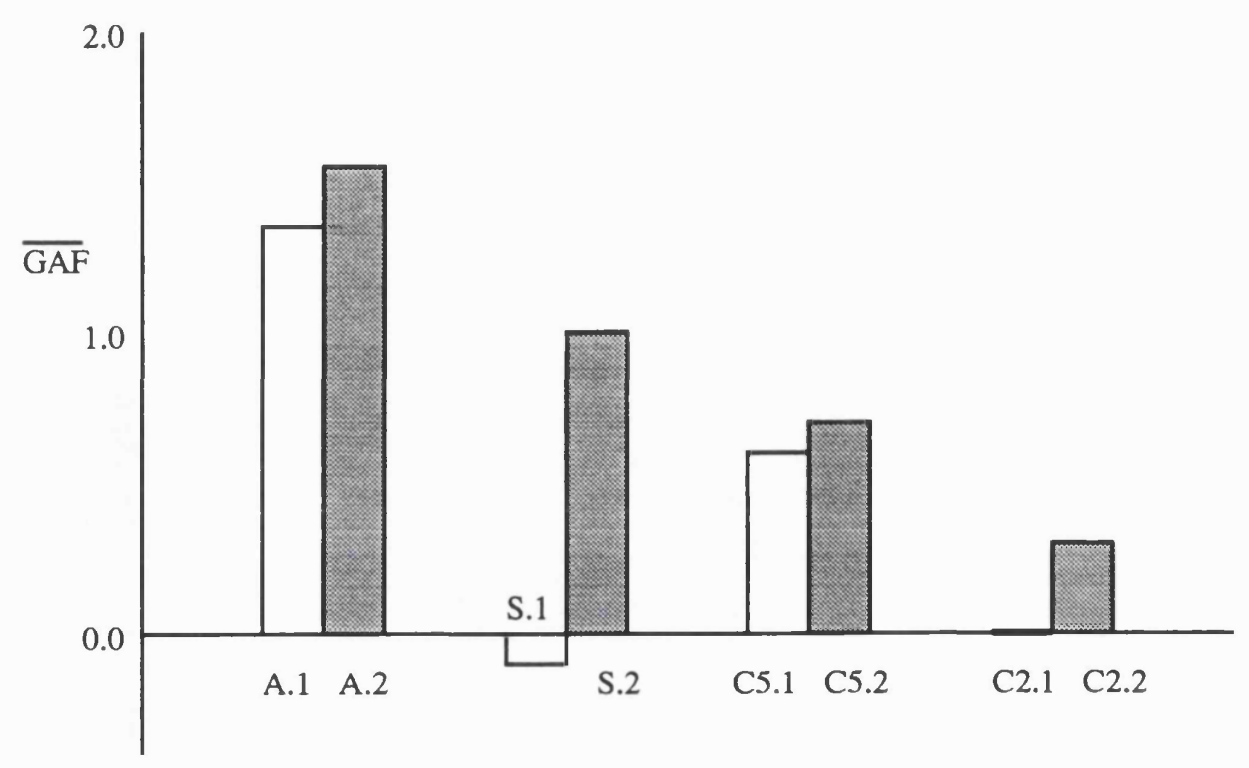

Figure 5.6: Comparative mean GAF achieved by UNIMEM and W-UNIMEM.

The W-UNIMEM program has produced a significant change to the concept networks generated from the cases with chromosomal abnormalities (see Figures 5.4 and 5.5). The effect of introducing feature salience to the evaluation and generalisation functions is to cause a filtering action in which extraneous links between cases with different banding defects are avoided. Figure 5.4 shows Network C5.2, formed by the cases with a duplication in the number 5 chromosome. There is a significant increase in the number of uncategorised cases (formerly 19, now 37), and a decrease in the number of concept nodes created (20 nodes as oppose to 31 nodes in Network C5.1). However, overall the number of concept nodes linking cases of the same structural defect is the same. In both networks (with and without the diagnostic model) 11 concept nodes have been generated which link cases with the same banding defect. The same kind of comparisons can be made between Network C2.1 and Network C2.2, generated by W-UNIMEM with the cases with a deletion in the number 2 chromosome (Figure 5.5). The number of extraneous groups has decreased whilst the number of uncategorised cases has grown (formerly 15, now 32 cases unclassified). This decrease in the number of extraneous links between cases effectively reduces the negative contribution to the mean group accuracy factors for both data sets of chromosomal abnormality cases. Figure 5.6 shows that the $\overline{G A F}$ has increased in both cases when compared to the networks generated by UNIMEM. The $\overline{G A F}$ for Network C5.2 is +0.687 , and the $\overline{G A F}$ for Network C2.2 is +0.337 . 


\subsection{Discussion}

The revised CBL algorithm now functions in a more informed manner than UNIMEM. The matching that governs evaluation and generalisation is now guided by domain knowledge rather than mere frequential appraisal. As well as improved matching, another consequence of having knowledge about feature salience is that concepts may now indicate the significance of individual features within their descriptions. This is illustrated by Table 5.3 which shows the generated descriptions of four concept nodes of Network S.2. Nodes N4 and N4.1 collectively group eight cases from the short-rib family of syndromes. The more general node description (N4) lists the permanent feature (chest rib-shortening severe), which is indicated as having high significance $\left(S_{4}\right)$, along with a secondary chest anomaly and a chest abnormality of low diagnostic power indicated by 0/C (features with a diagnostic significance regarded as occasional or common: levels 1 and 2 with respect to the LDDB listing). The sub-node (N4.1) lists a number of hand abnormalities which carry secondary significance along with some long-bone abnormalities which, individually, carry low significance. These general descriptions are consistent with those found in the literature for the short-rib family (i.e., these syndromes are characterised by severe rib-shortening, short stature and hand and/or feet abnormalities [30]). The descriptions of Nodes N5 and N5.1 are also consistent with the relative descriptions of SEDC and SEMD found in the literature. The more general node of the two (Node N5) suggests that epiphyseal abnormality is the key distinguishing feature of the higher group (a hard anomaly) which links cases of SEDC, whilst its sub-node (Node N5.1), which groups all three cases of SEMD, is characterised by metaphyseal abnormality (also ranked as a hard feature belonging to the significance set $S_{4}$ ).

An important point regarding the experiments reported in both this section and those of the previous chapter is that the diagnoses of the cases used to generate concept networks are known. The concept networks that have been created have been generated, to some extent, by empirical manipulation. Thus, the system does not form concepts independently; it is guided, or supervised, by the user. The operation of LDDB requires a similar type of empirical manipulation. To successfully use LDDB, the user (a physician) may be required to define a number feature combinations (of varying generality) before a satisfactory list of possible syndromes is returned. The key distinction between these two operations is that when using LDDB the physician does not know the diagnosis. 


\begin{tabular}{|c|c|c|c|c|}
\hline Node & Permanent & $S$ & Non-permanent & $S$ \\
\hline N4 & $\begin{array}{l}\text { (chest clavicles elongated) } \\
\text { (chest rib-shortening severe) }\end{array}$ & $\begin{array}{l}\mathrm{O} / \mathrm{C} \\
S_{4}\end{array}$ & (chest clavicles handlebar) & $S_{3}$ \\
\hline N4.1 & $\begin{array}{l}\text { (fibula diaphysis shortened) } \\
\text { (femur diaphysis shortened) } \\
\text { (humerus diaphysis shortened) } \\
\text { (humerus metaphysis-proximal } \\
\text { hypoplastic) } \\
\text { (hands length-of-metacarpals-phalanges } \\
\text { decreased-metacarpals) } \\
\text { (hands length-of-metacarpals-phalanges } \\
\text { decreased-phalanges) } \\
\text { (hands brachydactyly-phalanges } \\
\text { (proximal middle distal)) } \\
\text { (hands brachydactyly-metacarpals yes) }\end{array}$ & $\begin{array}{l}0 / \mathrm{C} \\
\mathrm{O} / \mathrm{C} \\
\mathrm{O} / \mathrm{C} \\
\mathrm{O} / \mathrm{C} \\
S_{3} \\
S_{3} \\
S_{3} \\
S_{3}\end{array}$ & & \\
\hline N5 & $\begin{array}{l}\text { (long-bones epiphyseal-abnormality } \\
\quad \text { abnormal) } \\
\text { (feet ossification-of-tarsals absent-talus) } \\
\text { (chest clavicles elongated) } \\
\text { (chest rib-post-cupping no) }\end{array}$ & $\begin{array}{c}S_{4} \\
S_{3} \\
\mathrm{O} / \mathrm{C} \\
\mathrm{O} / \mathrm{C}\end{array}$ & & \\
\hline N5.1 & $\begin{array}{c}\text { (long-bones metaphyseal-abnormality } \\
\text { abnormal) }\end{array}$ & $S_{4}$ & & \\
\hline
\end{tabular}

Table 5.3: Node N4, N4.1, N5 and N5.1 descriptions from Network S.2.

The creation of a manageable differential is based on skill and experience. Furthermore, the relative diagnostic importance of individual abnormalities may vary depending on context. That is to say, the user may adjust the index (boolean set of dysmorphic features) to the database whilst searching for a satisfactory differential diagnosis. In doing this the physician may, therefore, alter the emphasis of the index based on the returned syndromes in order to rule out syndromes, or refine the retrieved list. The physician constrains the operation of LDDB in the same way that a non-skilled user can constrain the generation of concepts with the described CBL algorithm. However, a dysmorphologist using LDDB adjusts the feature index according to a much greater breadth of specialist knowledge.

A second distinction between the supervision of LDDB and the CBL program is in the performance goal. In LDDB the goal is diagnosis. The use of a CBL system puts an emphasis on learning, i.e., the analysis of links made between previously uncategorised 
cases. Whilst the experiments with CBL have utilised diagnosed cases, the aim has been to demonstrate the mechanics of CBL when applied to dysmorphology. Because the cases are known a non-specialist has been able to achieve this aim. If the cases were undiagnosed then supervision would be required from a domain expert. However, the mechanics of operation would be the same. Thus, if a physician was operating in a learning mode, then the CBL program could be guided when operating on undiagnosed cases. In this instance, instead of altering an index the user would vary the system parameters in order to vary the emphasis, or salience, of relevant abnormality sets (i.e., $S_{3}$ and $S_{4}$ ). The aim would then be to derive sensible case groups in terms of the generated group descriptions. An experienced physician would have the required knowledge for such analysis. The node descriptions that are generated, such as those shown in Table 5.3, along with the descriptions of the cases linked to them, may thus provide a basis from which a new syndrome description may be proposed.

\subsection{Conclusions}

The previous discussion concluded that, given the depth of knowledge available, in order to operate with undiagnosed cases the CBL program should be supervised by a domain expert. The use of the CBL program would then involve some iterative adjustment of system parameters and case group analysis in order to derive a potential link between undiagnosed cases with a corresponding description. The CBL program thus provides a semi-automatic CBR system. It provides a dynamic case memory, a retrieval and storage algorithm (effectively the same function), and two matching functions (evaluation and generalisation) which are guided by a domain knowledge module and supervised by an expert. Figure 5.7 illustrates this architecture. This model represents a specialised view of the general CBR architecture that was described in Chapter 2 (Figure 2.5). In the CBL model, the retrieval algorithm specifically requires a hierarchical case memory organisation. Evaluation is then performed with every object that is retrieved through confined, recursive search. The list is not ranked or refined beforehand. The CBL model does not have an independent similarity assessment phase within its overall operation. Matching is performed in conjunction with search. The general CBR model incorporates a number of semi-independent program modules which operate in a more procedural manner. Salient indexes are defined, an object set is retrieved, this list is ranked, sim- 
ilarity assessment is performed, and the resulting evaluation decides where in memory the new case should be stored and whether any reorganisations should take place.

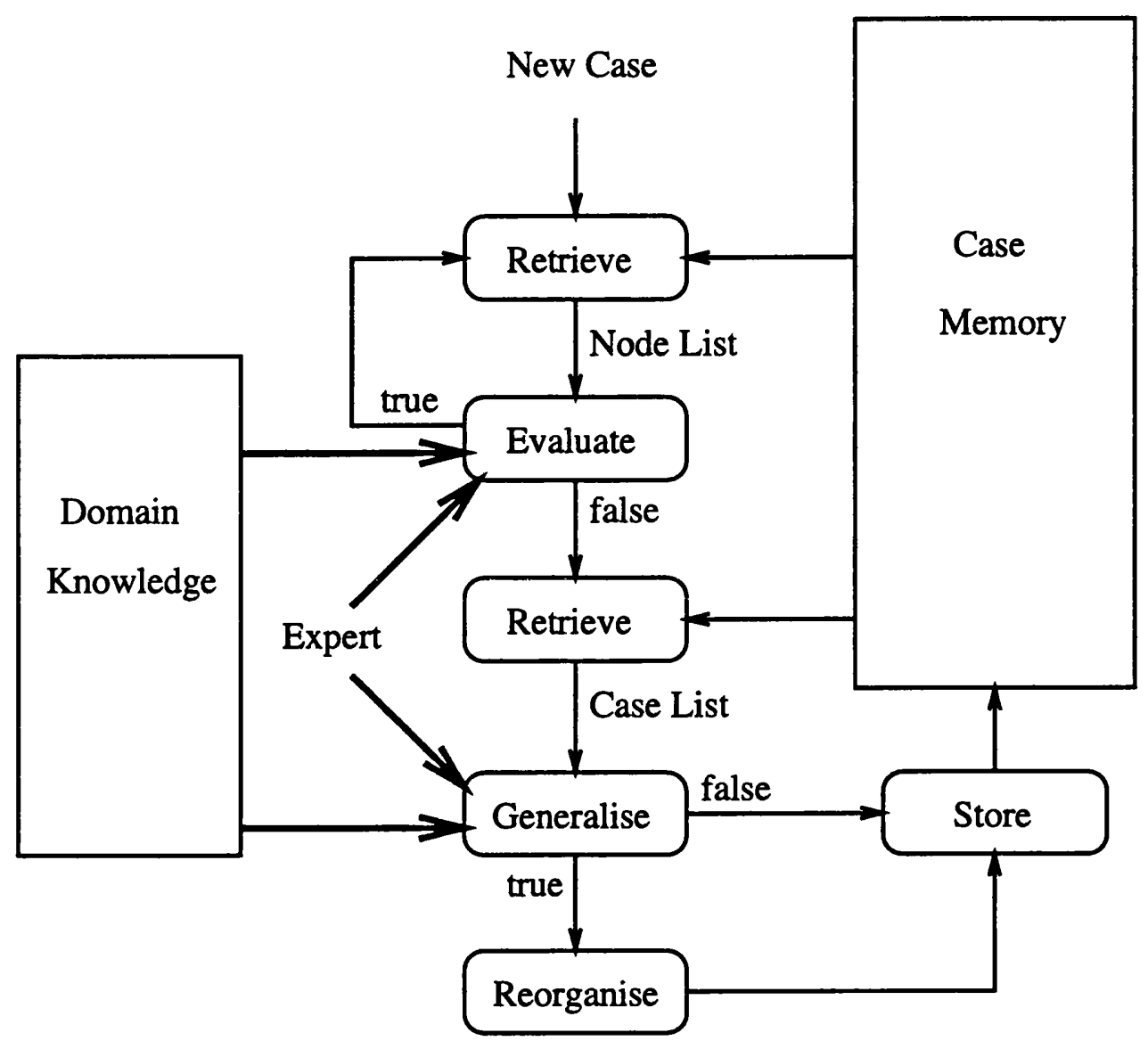

Figure 5.7: A general CBL system

The experiments performed with the CBL program have utilised a very artificial environment. Consultants are not generally presented with thirty or forty new patients at once. Whilst a physician will process new cases incrementally, cases are presented to the physician in a much larger time frame. Furthermore, the initial performance task is nearly always diagnosis. It is usual that when a diagnosis is not apparent the physician will then focus on any potential links with previously undiagnosed cases.

Whilst it has been demonstrated that the underlying dynamics of incremental concept formation are comparable to case-based learning there is a subtle distinction between the two. The emphasis of incremental concept formation systems is more concerned with the generation of conceptual categories from a training set of instances. The resulting network may then be tested against further cases whose categories are known. In terms of 
general case-based reasoning, the mechanics of learning are very similar, however, there is less overall emphasis on continual category update. Finding the best location within a structured case-base through indexing, retrieval and similarity assessment may well be sufficient. This is effectively what happens when a case is successfully diagnosed (against a known syndrome) by an expert. The syndrome description is not necessarily updated each time a new patient is diagnosed. This fact provides an argument against continual reorganisation of a dysmorphology case-base. Perhaps, therefore, in a more general CBR system dedicated to dysmorphology, in order to allow both diagnosis (finding the best location) and learning (analysis through reorganisation), it may be beneficial to make the processes of search, similarity assessment and memory update more independent. With such a model, the (expert) user could control, or guide, each independent process.

The conclusions of Chapter 4 noted certain weaknesses of the CBL algorithm with regard to similarity assessment. Matching based on frequency provides a poor model of the domain, and is particularly susceptible to the non-uniformity of case descriptions. The inclusion of domain knowledge within the system does alleviate these problems to some extent. In fact, the performance of W-UNIMEM, in terms of the accuracy in which it groups cases, is considerably better than UNIMEM. However, the matching functions still have weaknesses when viewed in the context of the actual similarity assessment that is performed by specialists. For instance, there is no contrast between anomalies that match and those that don't. In other words, dissimilar features do not contribute to the assessment of similarity between objects (i.e., no negative weight is assigned to unmatching features). For features of low significance (common and occasional) this is acceptable. However, unmatching features of higher significance can play an important role in the overall similarity between a patient and a syndrome. It was stated earlier that some abnormalities are so diagnostic to particular conditions that they are considered essential in order for a diagnosis to be confirmed. Knowledge of essential features is not available in this instance, as it is with the GENDIAG system, but the system does know which features have a relatively high diagnostic weight. As the CBL model currently stands, features listed for the objects being compared that belong to the most significant set $\left(S_{4}\right)$ or secondary set $\left(S_{3}\right)$ add support to the calculation of similarity if they match, but do not count against it if they do not. Thus, a situation could arise in which a case has two hard abnormalities, one of which matches a node description causing successful evaluation. If the other hard anomaly does not match the node description, then in real 
life this may provide strong evidence, or support, against the match. However, with the current CBL model no significance is assigned to dissimilarity, and evaluation would succeed irrespective of the hard anomaly that did not match.

Chapter 6 is concerned with similarity assessment. A model of similarity taken from the psychology literature, called the contrast model, is analysed with respect to the dysmorphology domain. The goal is to improve the performance of the CBL program by extending the functionality that assesses the similarity between two entities. The contrast model is considered for inclusion within the CBL program because it specifically takes into account both the matching and distinctive features of two entities when calculating a measure of similarity. Furthermore, it allows the matching to be biased, or asymmetrical. This maps well with an aspect of matching in dysmorphology, that the emphasis of similarity can alter depending on the performance goal, i.e., diagnosis or learning. An extended version of the contrast model is incorporated into the CBL program, which in turn facilitates an improved performance when utilised with the dysmorphology data sets.

\subsection{Summary}

- The two types of similarity assessment performed by UNIMEM, evaluation and generalisation, are improved by utilising a model of diagnostic significance from the London Dysmorphology Database.

- An improved version of the UNIMEM algorithm, called W-UNIMEM, which utilises the extended similarity functions, is designed and implemented. The W-UNIMEM algorithm incorporates additional parameters that allow the diagnostic weight of dysmorphic features to contribute to similarity assessment. The values to which these parameters are set have been derived through a combination of empirical judgement and some general rules given in the literature. Empirical judgement has been achievable by a non-specialist because the actual diagnoses of cases is known. It is concluded that operation with unknown cases should be supervised by a domain expert.

- W-UNIMEM achieves a greater mean group accuracy factor for all four dysmorphology data sets than was achieved by UNIMEM. Furthermore, the concepts that 
are generated by W-UNIMEM are also improved. Category information derived through W-UNIMEM incorporates feature sets that indicate the diagnostic significance of individual features.

- It is identified that distinctive features do not contribute to similarity assessment in the W-UNIMEM and UNIMEM models. However, this facet of matching is of considerable importance within the field of dysmorpology. 


\section{Chapter 6}

\section{A General Theory of Similarity}

\subsection{Introduction}

Earlier discussion highlighted that in complex medical domains it is not always intuitive to represent expert knowledge numerically. Physicians are generally uncomfortable with this notion. However, the employment of numerical formulae is common. Whilst not accepted as firm evidence in diagnosis, numerical computations can provide a compromise, allowing a computer system to assist a human expert. Chapter 2 described dysmorphology systems that use Bayes' formula and Dempster-Schafer theory (belief functions) to rank differential diagnoses. These two formulas are examples of statistical methods, and are widely used in medical informatics. This is despite the fact that they are dependent on certain statistical assumptions that do not necessarily hold in some fields of medicine, and certainly do not hold within dysmorphology. The work by Preus et al. employed numerical methods, albeit with a different emphasis on the performance task. This research was less concerned with diagnosis as such, and was more an investigation on the applicability of numerical methods as a clustering technique within multi-dimensional feature space. Again, as with statistical measures, the employment of dimensional metrics within the complex world of dysmorphology has its limitations, which are discussed in the following section.

Similarity assessment is a fundamental process to both diagnosis and learning in dysmorphology. A number of different methods of similarity assessment from dysmorphology computer systems were described in Chapter 2. These include ranking systems like SYNDROC, which employ a mathematical formula, and expert systems such as SDD, which 
use knowledge engineering techniques to arrive at a qualitative model of diagnosis ${ }^{1}$. The work presented here has favoured a compromise approach, and has adopted Trersky's set theoretical model of similarity assessment $[77,78]$ for inclusion within the CBR model. That is to say, the model of similarity that is employed in this research is a mathematical formula, albeit taken from the psychology literature. However, it is a theory specific to assessing similarity in contexts where dimensional comparison is not intuitive, and does not rely on statistical or probabilistic assumptions.

\section{Addendum}

The review sections 2.4.4, 2.4.5 and 2.4.6 highlight certain problems with previous applications of Bayes' rule to dysmorphology, and the use of statistical techniques in general. For example, Bayes' formula makes the assumption that all features are independent from each other and that the domain is closed in terms of the possible diseases. Both these factors cannot be assumed within dysmorphology. These problems, therefore, provide additional justification for disregarding Bayesian statistics in favour of the Tversky equation used with the C-UNIMEM program described in Chapter 6.

\subsection{Tversky's Theory of Similarity}

The theory of similarity put forward by Tversky was done so as an antithesis to previous numerical models in which objects represent points in coordinate space such that the observed dissimilarities between objects correspond to the metric distances between the respective points. A tenet of Tversky's work is that the assumption of representing similarity dimensionally, or with this type of metric, is open to question. Dimensional operators may be appropriate for certain stimuli, but not others. It may be that in certain situations a qualitative description best serves to represent objects under similarity assessment. In such circumstances, similarity would involve a comparison between features rather than a computation of metric distance between points. This would certainly

\footnotetext{
${ }^{1}$ Whilst SDD incorporates a model of similarity assessment, its operation also relies to some extent on numerical comparisons.
} 
seem to be consistent with the recognised inadequacies of applying distant metric formulae to problems of medical diagnosis. Dysmorphology provides additional problems also outlined by Tversky when mapping dimensional measures to similarity. In a dimensional model such as nearest neighbour analysis, similarity, or dissimilarity, is judged merely in terms of geometric distance $[17,27]$. Such measures are symmetric in the sense that there exists no bias in the assessment of whether two objects are the same or different. In dysmorphology, the higher order performance task can influence the type of similarity assessment. In attempting a diagnosis, the physician may try to prove that a patient has a particular syndrome by emphasising certain abnormalities that match. Alternatively, when analysing a new case with respect to a recognised syndrome family, and with a view to revising the category structure, the physician may emphasise significant dissimilar features exhibited by the patient (with reference to the relevant syndrome prototypes). In both instances, the similarity or dissimilarity between a patient and syndrome can be based merely on one very salient abnormality, sometimes regardless of the features that fail to co-occur between two cases. Dimensional distance metrics which do not account for the directional bias of the similarity process, or the contextual bias that can be attributed to individual features, thus, do not map ideally with the types of matching performed in dysmorphology. Tversky's model, on the other hand, specifically addresses the asymmetrical aspect of similarity. The contrast model does not operate in multi-dimensional space. It is an assessment between three overlapping feature sets: those features that match, those exhibited by the first case that are missing in the second case, and conversely those features exhibited by the second case that are missing in the first case. Trersky's model allows the contribution of these three feature sets to the overall similarity to be varied according to the emphasis of the match. Furthermore, the contrast model incorporates an operator to account for the contextual salience of individual features. That is to say, individual features may take on additional relevance if the context of the match dictates. The mental retardation example from dysmorphology demonstrates this idea. In the general population (of dysmorphic syndromes), mental retardation is so frequent that it carries low significance when attempting to establish an initial differential diagnosis. This task is relatively context free. However, when specifcally investigating a potential diagnosis, the fact that an infant has mental retardation can, in the context of the hypothesis, be very important. Whilst the contrast model incorporates a context sensitive operator (or function) specifically to account for this 
additional type of bias, dimensional metrics do not. The following sections present the basic principals of the contrast model. The ensuing discussion relates these ideas to the dysmorphology domain.

\subsubsection{The Contrast Model}

Let $\Delta=\{a, b, c \ldots\}$ be the domain of objects under study. Assume that each object in $\Delta$ can be represented by a set of features or attributes, and let $A, B, C$ denote the set of features associated with the object $a, b, c$ respectively. Let $s(a, b)$ be a measure of the similarity between $a$ and $b$. The theory assumes that the similarity measure $s$ is expressed as a function of three arguments: $A \cap B$, the features that are common to both $a$ and $b ; A-B$, the features that belong to $a$ but not to $b ; B-A$, the features that belong to $b$ but not to $a$. Given a similarity scale $S$, and a non-negative scale $f$, the observed similarity between two objects $a$ and $b$ is denoted by:

$$
S(a, b)=\theta f(A \cap B)-\alpha f(A-B)-\beta f(B-A)
$$

where $\theta, \alpha, \beta \geq 0$, and $f$ and $S$ are interval scales. Thus, Tversky's representation of similarity is a linear combination, or a contrast, of the measures of the common and distinctive features. For this reason it is referred to as the contrast model. The contrast model defines varying scales of similarity $(S)$ that are characterised by different values of the parameters $\theta, \alpha$ and $\beta$. For example, if $\theta=1, \alpha=\beta=0$, then $S(a, b)=f(A \cap B)$; that is, the similarity between objects is determined only by their common features. Alternatively, a match between two objects may be defined in terms of their distinctive features only by making $\theta=0, \alpha=\beta=1$, which gives a measure for dissimilarity (or negative similarity), i.e., $-S(a, b)=f(A-B)+f(B-A)$. The scale $f$ is intended to reflect the salience, or prominence, of the various features, i.e., it decides the contribution, or weight, that a particular (common or distinctive) feature provides to the similarity between objects.

\subsubsection{Asymmetry and Focus}

A major thrust of Tversky's theory is that similarity should not be assumed to be symmetric. Furthermore, similarity relationships have directionality. Assessment is between a subject and a referent, the choice of which depends on the relative salience of the objects, although, generally the referent is a prototype. Thus, the direction of comparison 
refers to whether matching is meant to determine the degree to which $a$ and $b$ are similar, or the degree to which $a$ is similar to $b$. In the latter case, the task is directional, or asymmetrical. If $s(a, b)$ is interpreted as the degree to which $a$ is similar to $b$, then $a$ is the subject and $b$ is the referent. Tversky assumes that in this type of comparison focus is on the subject, and thus, features of the subject are weighted more heavily than the features of the referent. This is represented in the contrast model by making $\alpha>\beta$. Consequently, similarity is reduced more by the distinctive features of the subject than by the distinctive features of the referent. Tversky calls this the focusing hypothesis.

\subsubsection{Similarity versus Difference}

Along with a direction of comparison, there is also a focusing distinction between the task of establishing similarity and the task of establishing dissimilarity. An increase in the measure of the common features increases similarity and decreases difference, whereas an increase in the measure of the distinctive features decreases similarity and increases difference. However, the relative weight assigned to the common and distinctive features may differ in the two tasks. In assessment of similarity, more weight is attributed to common features, i.e., the relative weight of non-matching features will be less. Thus, in the contrast model this would be represented by making $\theta$ relatively large in comparison to $\alpha$ and $\beta$. Alternatively, if the task is to prove that two objects are dissimilar then the relative weight attributed to the distinctive features may increase accordingly. In this instance $\alpha$ and $\beta$ would be relatively large compared to $\theta$.

\subsubsection{Similarity in Context}

Similarity depends on context, or frame of reference. In Tversky's model, context may be accounted for through the feature space, i.e., certain features that are relevant given the context of the similarity assessment will be assigned weight in the match, whilst those that are not will be assigned zero weight. Thus, the value $f$ denoting salience in the contrast model is a context dependent value. Tversky's model assumes that such contextual knowledge is available, and the salience function, $f$, defined by equation 6.1 , is generic to both the common and distinctive features. 


\subsubsection{The Diagnosticity Principle}

The salience, or measure of a feature can have two factors: diagnostic and intensive. The intensity factor refers to scale, e.g., severe, moderate or mild. Diagnostic factors refer to classificatory significance. The diagnosticity principle is defined as the relationship between similarity and the grouping of individual objects into clusters described by category information (concept descriptions). It states that clusters are described so as to maximise both the similarity of the objects within them and the dissimilarity of objects from different clusters, and that an alteration to a cluster will result in a change of the diagnostic value of the features that describe it.

\subsubsection{Discussion}

Tversky's initial premise that not all features within certain domains are intuitively represented by dimensional operators is certainly consistent with the imperfect compromise that is made when medical knowledge is represented numerically. In dysmorphology, this is certainly the case, and the diagnosticity principle holds in this instance. Abnormal features are described in qualitative terms and can have an intensity value (e.g., severe rib shortening). Individual cases are linked, or clustered, according to generalised (prototypical) descriptions, i.e., syndromes. Within a syndrome description individual features do take on classificatory significance (or diagnostic relevance) which can alter as more cases become diagnosed (and join the cluster). For example, in rare syndromes for which only a few cases are known, the diagnostic significance of certain abnormalities may be unclear. For syndromes which have many cases (e.g., Down syndrome) an understanding of the intra-class significance of individual features increases as more cases join the group. The diagnosticity principle does not map perfectly to the dysmorphology domain, however. The theory does not account for a relationship between the two factors mentioned: diagnosticity and intensity. In dysmorphology, there is a definite relationship between these factors. The intensity of an abnormality can increase its classificatory significance. For example, certain syndromes within the short-rib family are characterised by severe rib-shortening. This has such classificatory significance that there would be doubt about a case which only exhibited mild rib-shortening. Also, the diagnosticity principle assumes that inter-class dissimilarity is maximised by group descriptions. This goal is characteristic of clustering systems that aim to generate disjoint partitions between concepts. It 
is not evident that dysmorphology specialists make this assumption. Rather, it is noted that disorders are not necessarily mutually exclusive [87].

Asymmetrical matching is also apparent within dysmorphology. During diagnosis there is clearly a subject (the patient) and a referent (a syndrome) which is described in terms of prototypical features. In this instance, similarity assessment is directional. It determines by how much a patient is similar to a syndrome. However, if the performance task has a learning goal, i.e., when two or more undiagnosed cases are being compared with a view to establishing a new syndrome description, then the similarity assessment may be more balanced. In this case the referent would also be a patient, not a syndrome.

It has already been stated that if a syndrome is suspected then the significance of patient features may take on additional focus, or bias (cf., the focusing hypothesis). In this sense the physician attempts to prove that the subject is more similar to the referent than the referent is dissimilar to the subject. Thus, Tversky's theory that there is a distinction between the task of proving similarity and the task of proving dissimilarity is true in dysmorphology. An assessment of dissimilarity may occur when the higher order goal is learning and the physician believes that a case belongs to a general syndrome family, but suspects that the patient may represent a new variation of the disease. Emphasis may then be assigned to the distinctive features of the case. The theory also indicates that similarity is reduced more by the distinctive features of the subject than by the distinctive features of the referent. In dysmorphology, if a patient does not exhibit one of the principal features listed by a syndrome then the hypothesis is weakened, but not eliminated. However, if the patient has a significant abnormality not known to be associated with the syndrome, the match is weakened much more considerably (i.e., $\alpha>\beta)$.

It was noted earlier how the context of a match can be important in dysmorphology (the mental retardation example). Tversky's view of contextual feature salience is also consistent with the concept of occasional features. Such abnormalities represent features of low significance in the context a particular hypothesis. Trersky's theory states that not all features of the feature space will necessarily contribute to similarity depending on the context of the match, and those that don't should be attributed zero weight. Occasional features can be ignored (attributed zero weight) depending on the performance goal and the subsequent directionality of the match. For instance, if the task is diagnosis, emphasis is put on the common features exhibited by the subject and the syndrome $(A \cap B)$. 
Occasional features will support the hypothesis in this instance and are attributed weight, albeit of a relatively small value. However, distinctive features of the referent $(B-A)$ that are known to be occasional may be ignored. Thus, the context of the match can alter the bias attributed to features depending on whether they are common or distinctive.

As a summary to this discussion of Tversky's model, the following three types of matching that occur within dysmorphology are defined and described with respect to the contrast model:

- Diagnosis. This is the primary type of similarity assessment performed by specialists and involves a directional match in which the common features of the patient and syndrome are attributed greater emphasis than the distinctive, or unmatching features $(\theta>\alpha, \beta)$. In this sense the physician attempts to prove the similarity between the case and the syndrome. Because the task of similarity takes precedence over dissimilarity, when the set of distinctive features includes abnormalities of relatively high diagnostic weight the overall similarity is reduced more by the distinctive features of the subject than the referent $(\alpha>\beta)$.

- Learning in isolation. Sometimes two undiagnosed cases that stand isolated with respect to syndrome nomenclature may exhibit sufficient similarity to suggest a new condition. In this instance, both the subject and the referent involved in the match are cases. Thus, the direction of matching may be more balanced $(\theta=\alpha=\beta)$. However, when this actually occurs, a new patient is usually compared to a previous case recorded in the literature. The previous case then takes on the role of referent, and the matching, which is a task of similarity, can therefore be biased in the same way as when a diagnosis is performed, with more emphasis put on the common anomalies $(\theta>\alpha=\beta)$.

- Referential learning. Whilst the above situation of matching isolated cases does occur, new syndromes are more often established with regard to context, or frame of reference. That is to say, new conditions are more generally defined through category reorganisation within a known syndrome group. It may become apparent (with increasing evidence) that a number of cases previously thought to be distinct, are in fact examples of the same condition. A new, more general syndrome may then be described (or merged) from the relevant cases. Alternatively, cases that were previously linked together may be identified (at a later date) as separate man- 
ifestations of a related disorder, in which case corresponding syndrome descriptions may be distinguished (or split up). Thus, learning within the context of a known syndrome group can involve both the task of similarity and dissimilarity. Reorganisation of syndrome descriptions can involve proving two distinct conditions are so similar to each other that they are in fact the same syndrome. On the other hand, a dysmorphologist may attempt to prove that a recorded syndrome links cases of different diagnoses by emphasising the dissimilarity between them. With reference to the contrast model, the relevant weight attributed to the common and distinctive features will subsequently depend on the performance task, similarity or dissimilarity.

\subsection{Integrating the Contrast Model within CBL}

The matching performed by the CBL algorithm corresponds to alternative symmetries of comparison. The evaluation process, which is dependent on the system parameter $E_{t}$, matches a new case to a prototype node with the same directionality as diagnosis. Currently, unmatching features carry no weight in the comparison $(\alpha=\beta=0)$. This represents an extreme variation of the contrast model, such that, in trying to prove the similarity of the subject to the referent, the distinctive features of both are totally ignored. The generalise function, which is dependent on a separate parameter $G_{t}$, represents a more symmetrical match between two individual cases. This can be viewed as learning in which the task is similarity. When generalisation takes place between the input case and a previous case that is stored at the root node (therefore isolated from known groups) then this corresponds to learning in isolation. A generalisation that takes place between the input case and a case linked to a node corresponds to referential learning (matching is performed in terms of the features that are distinctive from the parent node). However, in this instance, the dominant process is similarity rather than dissimilarity. The CBL program learns, therefore, through a task of similarity and merging, not by calculating the dissimilarity between cases in order to make them distinct.

This section reports on a further modification to the CBL program which incorporates an extended version of the contrast model. As with Tversky's model, both common and distinctive features are accounted for during similarity assessment (in evaluation and generalisation). Also, the salience of features within the three respective sets: $A \cap B, A-B$ 
and $B-A$, contributes to the matching process. The definition of $f$ in equation 6.1 implies a generic function that can vary the salience of the common and distinctive feature sets according to context. The extended contrast model explicitly allows context to be taken into account by defining an independent salience function for each of the three common or distinctive feature sets.

\subsubsection{The Extended Contrast Model}

With the contrast model, as shown in equation 6.1, the salience of individual features with respect to overall similarity is determined by the function $f$. The $f$ function is a generic operator that requires knowledge of feature salience with respect to the application domain. The model of diagnostic significance employed by the W-UNIMEM program provides such knowledge. According to the definition, the $f$ function is generic across all three feature sets: $A \cap B, A-B$ and $B-A$. It has already been shown that the emphasis of the match can influence the contribution of these three feature sets to the overall assessment of similarity, and the relative contribution is controlled by the parameters $\theta, \alpha$ and $\beta$. It was also noted that feature salience can be dependent on context (e.g., in asymmetrical matches, occasional features of the distinctive set $B-A$ can be assigned zero weight). Furthermore, the $f$ function is meant to reflect context. Thus, if the same $f$ function is to operate with each of the three contributing feature sets, it must be informed of the matching context in order to vary the associated weights accordingly. The extended version of the contrast model allows salience to be defined according to both emphasis (directionality) and context. In the extended contrast model, the bias of similarity can, as before, be varied through the parameters $\theta, \alpha$ and $\beta$. Additionally, the contribution of feature salience according to the context of the match is facilitated through an extended definition of the salience function $f$.

In the extended contrast model shown by equation 6.2 , similarity is calculated according to the level of significance of specific anomalies (denoted by $S_{1} \ldots S_{4}$ ), the emphasis of the match (which is either evaluation or generalisation), and whether the relevant features are common or distinctive (i.e., $A \cap B, A-B$ or $B-A$ ).

$$
S(a, b)=\theta f_{i}(A \cap B)-\alpha f_{j}(A-B)-\beta f_{k}(B-A)
$$

The three salience functions: $f_{i}, f_{j}$ and $f_{k}$, represent alternative assignments of feature significance for each feature set. Thus, they allow the weight of individual features 
(denoted by $W_{1} \ldots W_{4}$ ) to be varied according to context (although weights are still defined according to the four significance sets: $\left.S_{1} \ldots S_{4}\right)$. For example, if the match is asymmetrical, i.e., the evaluation of a case to a node, then it is feasible that occasional features that belong to the set $B-A$ (the distinctive features of the referent) may be ignored. Thus, whereas occasional features add support if they are listed amongst the common features $(A \cap B)$ and the corresponding feature salience can be positive $\left(W_{i}>0\right.$, where $i \in S_{4} \cup S_{3} \cup S_{2} \cup S_{1}$ ), occasionals that are within the set of distinctive features of the referent $(B-A)$ may be ignored $\left(W_{k}=0\right.$, where $\left.k \in S_{2} \cup S_{1}\right)$.

\subsubsection{The C-UNIMEM Algorithm}

Figure 6.1 shows a new version of the CBL program, called C-UNIMEM, which incorporates the extended contrast model described above. This program varies from $W$ UNIMEM at two control points. The Evaluation-Weight function has been replaced by a new function, Evaluation-Contrast, which incorporates the extended contrast model shown in Equation 6.2 with three parameters representing the three feature sets: $A \cap B(\mathrm{H}), A-B(\mathrm{~K})$ and $B-A\left(\mathrm{~K}^{\prime}\right)$. In this instance, the input case (I) represents the subject (a) and the node $(N)$ is the referent (b). Another important difference between C-UNIMEM and W-UNIMEM, is that C-UNIMEM does not restrict its matching processes to hard abnormalities (denoted by $S_{4}$ ) and secondary features (denoted by $S_{3}$ ). W-UNIMEM achieved this by setting the weights corresponding to features of lower significance (i.e., sets $S_{1}$ and $S_{2}$ ) to nil. With C-UNIMEM, similarity is assessed as a contrast between common and distinctive features rather than a simple summation of combined weight for common features of high significance. With this model in place, occasional features and features of low significance may contribute to the overall similarity depending on the emphasis and context of the match.

The generalise function has also been modified. It now incorporates the function Generalise-Contrast in the place of the Generalise-Weight function used by WUNIMEM. This also incorporates the extended contrast model of equation 6.2, and, as with the revised evaluation function, takes three parameters corresponding to the common and distinctive feature sets of the subject and referent. 
C-Unimem(N, I, F)

Let $\mathrm{G}$ be the set of features stored on $\mathrm{N}$.

Let $\mathrm{H}$ be the features in $\mathrm{F}$ that match features in $\mathrm{G}$

Let $\mathrm{K}$ be the features in $\mathrm{F}$ that do not match features in $\mathrm{G}$.

Let $K^{\prime}$ be the features in $G$ that do not match features in $F$.

If $\mathrm{N}$ is not the root node,

Then If Evaluation-Contrast(H, K, K') returns TRUE,

or Evaluate( $N, H, K)$ returns TRUE,

Let $\mathbf{S}$ be the empty list.

Then return the empty list.

For each child $\mathrm{C}$ of node $\mathrm{N}$,

If $\mathrm{C}$ is indexed by a feature $k$ in $\mathrm{K}$ and $k \in S_{4}$,

Then let $S$ be Union(S, Unimem(C, I, K)).

If $S$ is the empty list,

Then for each instance $J$ at node $N$,

Let $S$ be Union(S, Generalise(N, J, I, F)).

If $S$ is the empty list,

Then store $\mathrm{I}$ as an instance of node $\mathrm{N}$ with features $\mathrm{K}$.

Return N.

Generalise(N, J, I, F)

Let $\mathrm{G}$ be the features in instance $\mathrm{J}$.

Let $\mathrm{H}$ be the features in $\mathrm{F}$ that match features in $\mathrm{G}$

Let $\mathrm{K}$ be the features in $\mathrm{F}$ that do not match features in $\mathrm{G}$.

Let $K^{\prime}$ be the features in $G$ that do not match features in $F$.

If Generalisation-Contrast(H, $K, K^{\prime}$ ) returns TRUE,

Then create a new child $\mathrm{C}$ of node $\mathrm{N}$ with features $\mathrm{H}$.

Remove $\mathrm{J}$ as an instance of $\mathrm{N}$.

Let $G^{\prime}$ be the features in $\mathbf{G}$ that are not in $\mathrm{H}$.

Let $F^{\prime}$ be the features in $F$ that are not in $H$.

Store $\mathrm{J}$ as an instance of $\mathrm{C}$ with features $\mathrm{G}^{\prime}$.

Store $\mathrm{I}$ as an instance of $\mathbf{C}$ with features $\mathrm{F}^{\prime}$.

Return C.

Figure 6.1: Top level C-UNIMEM algorithm and generalise function. 


\subsubsection{Results}

A consequence of the extended definition for the $f$ function is that there are now three sets of weight settings for each match rather than one. This allows contextual bias to be attributed to the relevant feature sets. The concept network shown in Figure 6.4 was generated by C-UNIMEM with the Acrocephalosyndactyly cases and the parameter settings shown in Figures 6.2 (evaluation) and 6.3 (generalisation). Figure 6.2 shows that weight is attributed to features of low significance $\left(W_{1}, W_{2}=1\right)$ if the emphasis of the match is asymmetrical (i.e., diagnosis, or evaluation) and the features are common to both the subject and the referent, but no weight $\left(W_{1}, W_{2}=0\right)$ contributes to the overall similarity if the features are distinctive. Thus, occasional features add support to the match if they are common, but do not count against the match if they are distinctive.

$$
\begin{array}{r}
\theta=1.0, \quad \alpha=0.6, \quad \beta=0.4, \quad E_{t}=9.0 \\
f_{i} \rightarrow W_{i}= \begin{cases}7 & \text { if } i \in S_{4} \\
3 & \text { if } i \in S_{3} \\
1 & \text { otherwise }\end{cases} \\
f_{j} \rightarrow W_{j}= \begin{cases}7 & \text { if } j \in S_{4} \\
3 & \text { if } j \in S_{3} \\
0 & \text { otherwise }\end{cases} \\
f_{k} \rightarrow W_{k}= \begin{cases}7 & \text { if } k \in S_{4} \\
3 & \text { if } k \in S_{3} \\
0 & \text { otherwise }\end{cases}
\end{array}
$$

Figure 6.2: Evaluation contrast parameters: Acrocephalosyndactyly group.

The parameter settings shown in Figures 6.2 and 6.3 concur with the general guidelines discussed in Chapter 5. For evaluation, the combination of one hard anomaly plus one or two secondary features will provide enough weight for the evaluation threshold to be reached. Also, as with previous experiments, for the generalisation process the generalisation threshold is set equal to the weight assigned to dysmorphic features of the highest significance $\left(S_{4}\right)$. However, with the extended contrast model, features of lower 


$$
\begin{gathered}
\theta=1.0, \quad \alpha=0.3, \quad \beta=0.3, \quad G_{t}=7.0 \\
f_{i}, f_{j}, f_{k} \rightarrow W= \begin{cases}7 & \text { if } i, j, k \in S_{4} \\
3 & \text { if } i, j, k \in S_{3} \\
1 & \text { otherwise }\end{cases}
\end{gathered}
$$

Figure 6.3: Generalisation contrast parameters: Acrocephalosyndactyly group.

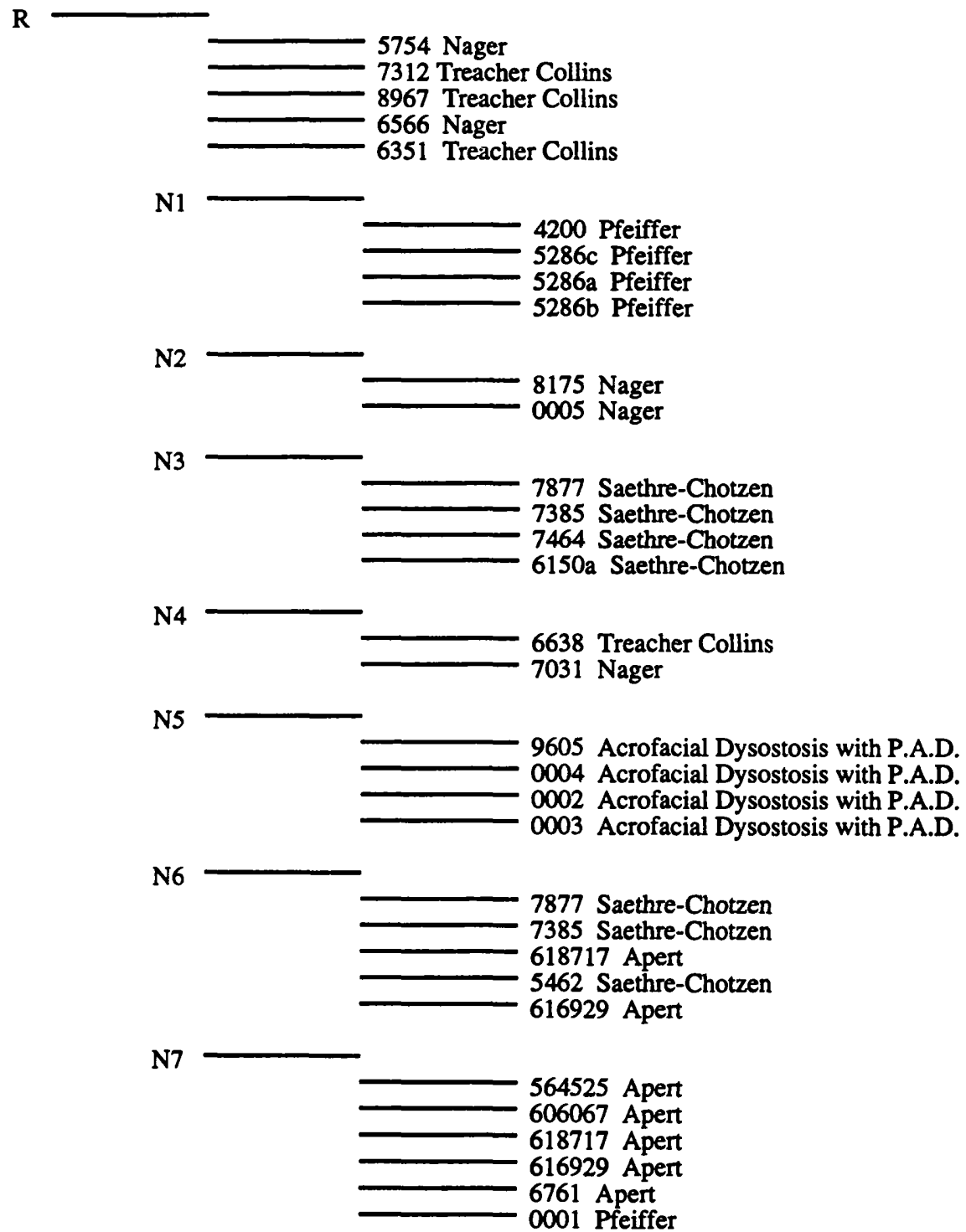

Figure 6.4: Network A.3: Concept hierarchy generated by the Acrocephalosyndactyly data set. 


\begin{tabular}{|c|c|c|c|c|c|}
\hline$A \cap B$ & $S_{i}$ & $A-B$ & $S_{j}$ & $B-A$ & $S_{k}$ \\
\hline $\begin{array}{c}\text { (face mandible } \\
\text { small-mandible/micrognathia) }\end{array}$ & $S_{2}$ & $\begin{array}{c}\text { (hands fingers } \\
\text { skin-syndactyly-of-fingers) }\end{array}$ & $S_{3}$ & $\begin{array}{c}\text { (eye-structures eyelids } \\
\text { ectropion-of-eyelids) }\end{array}$ & $S_{3}$ \\
\hline $\begin{array}{c}\text { (upper-limbs forearm } \\
\text { hypoplastic-or-sbsent-ulns) }\end{array}$ & $S_{4}$ & $\begin{array}{c}\text { (ears external-ears } \\
\text { simple-ears) }\end{array}$ & $S_{2}$ & $\begin{array}{c}\text { (face malar-region } \\
\text { flat-malar-region) }\end{array}$ & $S_{3}$ \\
\hline $\begin{array}{c}\text { (hands fingers } \\
\text { absent-fingers-or-oligodactyly) }\end{array}$ & $S_{4}$ & $\begin{array}{c}\text { (mouth general } \\
\text { microstomis) }\end{array}$ & $S_{3}$ & $\begin{array}{c}\text { (oral-region palate } \\
\text { cleft-palate) }\end{array}$ & $S_{3}$ \\
\hline $\begin{array}{c}\text { (feet toes absent-toes) } \\
\text { (ears external-ears } \\
\text { low-set-ears) }\end{array}$ & $S_{3}$ & $S_{2}$ & & \\
\hline & & $\begin{array}{c}\text { (upper-limbs elbow } \\
\text { elbow-joint-synostosis) }\end{array}$ & $S_{3}$ & & \\
\hline & & $\begin{array}{c}\text { (ears hearing } \\
\text { deafnest-conductive) }\end{array}$ & $S_{3}$ & & \\
\hline
\end{tabular}

Table 6.1: Contrast between Case 5754 and Node N5 of Network A.3.

significance (i.,e $S_{1} \cup S_{2}$ ) also contribute to similarity assessment. This contribution reflects the contextual relevance of lower order anomalies and is facilitated by the relevant $f$ functions. For evaluation, features belonging to significance sets $S_{1}$ and $S_{2}$ are attributed weight if they pertain to the common feature set, $A \cap B$. If they belong to either of the distinctive feature sets then they are attributed no weight. Whilst the parameter settings for $E_{t}, G_{t}$ and the respective values for $W_{4}$ and $W_{3}$ follow the general guidelines mentioned above, the contrast parameters: $\theta, \alpha$ and $\beta$, account for the additional bias or symmetry to each match. For evaluation, which is asymmetrical matching in which the distinctive features of the subject take precedence over the distinctive features of the referent, the relative contrast parameters are set such that $\theta>\alpha>\beta$. The evaluation threshold would normally be reached if the common features of the subject and referent included one hard anomaly plus a secondary feature, or by the combined weight of sufficient secondary anomalies, but now distinctive features can contribute negative weight to the overall similarity. The generalisation conditions shown in Figure 6.3 show a more balanced contrast between common and distinctive features, as would be expected when both the subject and the referent are cases. In this function, the emphasis of the match is facilitated by contrast parameter settings such that $\theta>\alpha=\beta$. Furthermore, the context of generalisation is learning, and thus, the contextual salience of feature sets is more balanced than that of evaluation (i.e., $f_{i}=f_{j}=f_{k}$ ).

When matching between cases is performed only as a function of common abnormali- 
ties, some matches may satisfy the necessary threshold despite the existence of significant distinctive features. This is true of both UNIMEM and W-UNIMEM. The introduction of a contribution in the match from distinctive anomalies should, overall, produce a more balanced assessment of similarity. Thus, one difference that one would expect to see between the networks formed by version 2 and version 3 of the CBL algorithm, is a further decrease in the number of extraneous links. This effect is shown by Network A.3 (Figure 6.4) with regard to Case 5754. In Network A.2 (Figure 5.2), Case 5754 (Nager) was grouped with the four cases of Acrofacial Dysostosis with post-axial defects. In Network A.3 this case is unclassified. The input ordering of the cases is such that when Case 5754 evaluates against Node N5 of Network A.3, the common and distinctive abnormalities are those listed in Table 6.1. Despite the fact that there are no distinctive abnormalities of the highest significance $\left(S_{4}\right)$, the combined weight of the distinctive secondary features is sufficient to prevent the evaluation succeeding. The resulting similarity score, $s(a, b)$, is 7.2, which fails to reach the evaluation threshold $\left(E_{t}=9.0\right)$. In fact, Case 5754 fails to evaluate against any node, or instance linked to the root, and is stored at the root (and is therefore unclassified).

$$
\begin{array}{r}
\theta=1.0, \quad \alpha=0.6, \quad \beta=0.4, \quad E_{t}=15.0 \\
f_{i} \rightarrow W_{i}= \begin{cases}12 & \text { if } i \in S_{4} \\
3 & \text { if } i \in S_{3} \\
1 & \text { otherwise }\end{cases} \\
f_{j} \rightarrow W_{j}= \begin{cases}12 & \text { if } j \in S_{4} \\
3 & \text { if } j \in S_{3} \\
0 & \text { otherwise }\end{cases} \\
f_{k} \rightarrow W_{k}= \begin{cases}12 & \text { if } k \in S_{4} \\
3 & \text { if } k \in S_{3} \\
0 & \text { otherwise }\end{cases}
\end{array}
$$

Figure 6.5: Evaluation contrast parameters: skeletal dysplasias group.

The network generated with the skeletal dysplasias cases is shown in Figure 6.7. This was formed with the parameter settings shown in Figures 6.5 and 6.6. The re- 


$$
\begin{gathered}
\theta=1.0, \quad \alpha=0.3, \quad \beta=0.3, \quad G_{t}=12.0 \\
f_{i}, f_{j}, f_{k} \rightarrow W= \begin{cases}12 & \text { if } i, j, k \in S_{4} \\
3 & \text { if } i, j, k \in S_{3} \\
1 & \text { otherwise }\end{cases}
\end{gathered}
$$

Figure 6.6: Generalisation contrast parameters: skeletal dysplasias group.

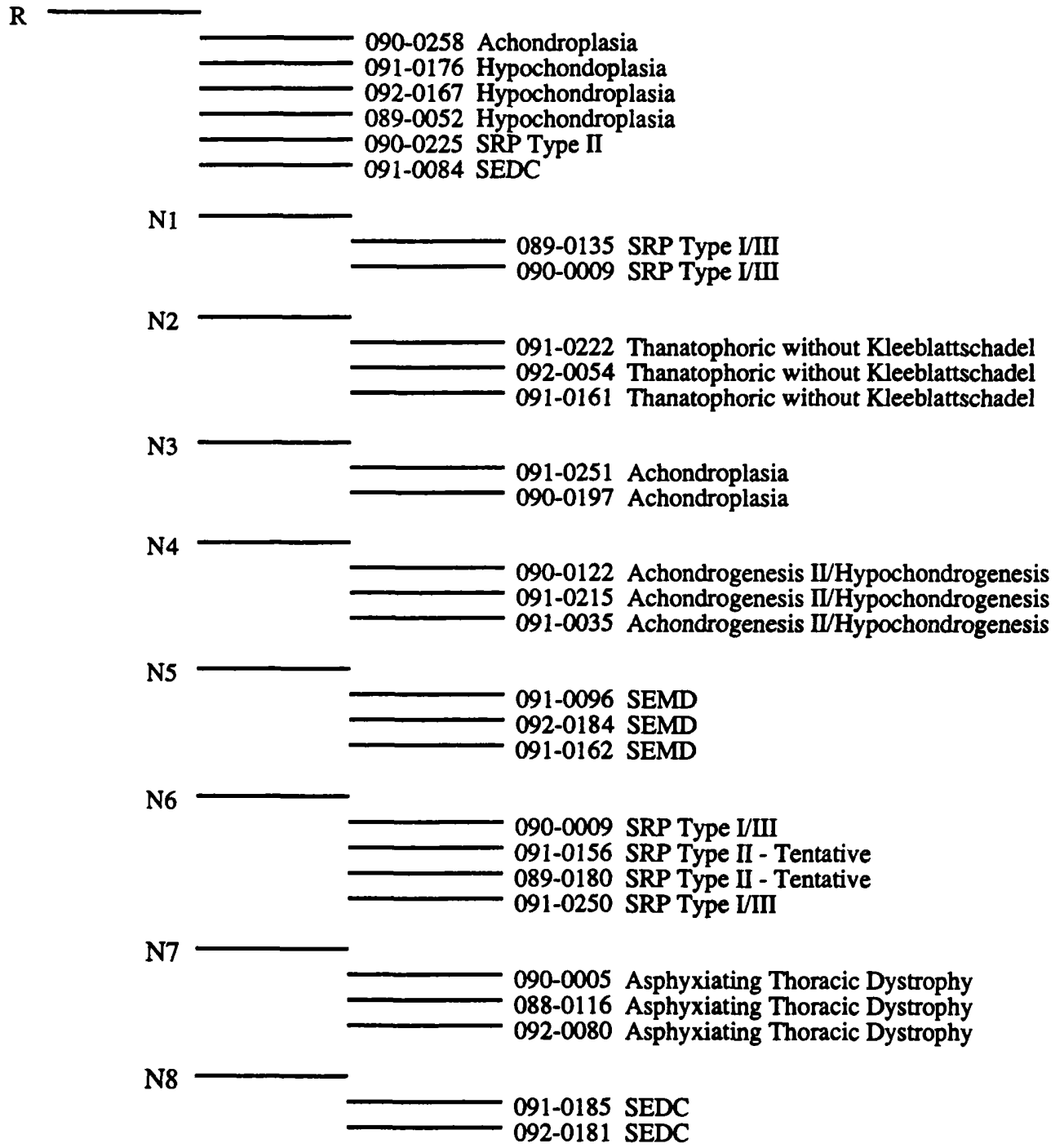

Figure 6.7: Network S.3: Concept hierarchy generated by the skeletal dysplasias data set. 


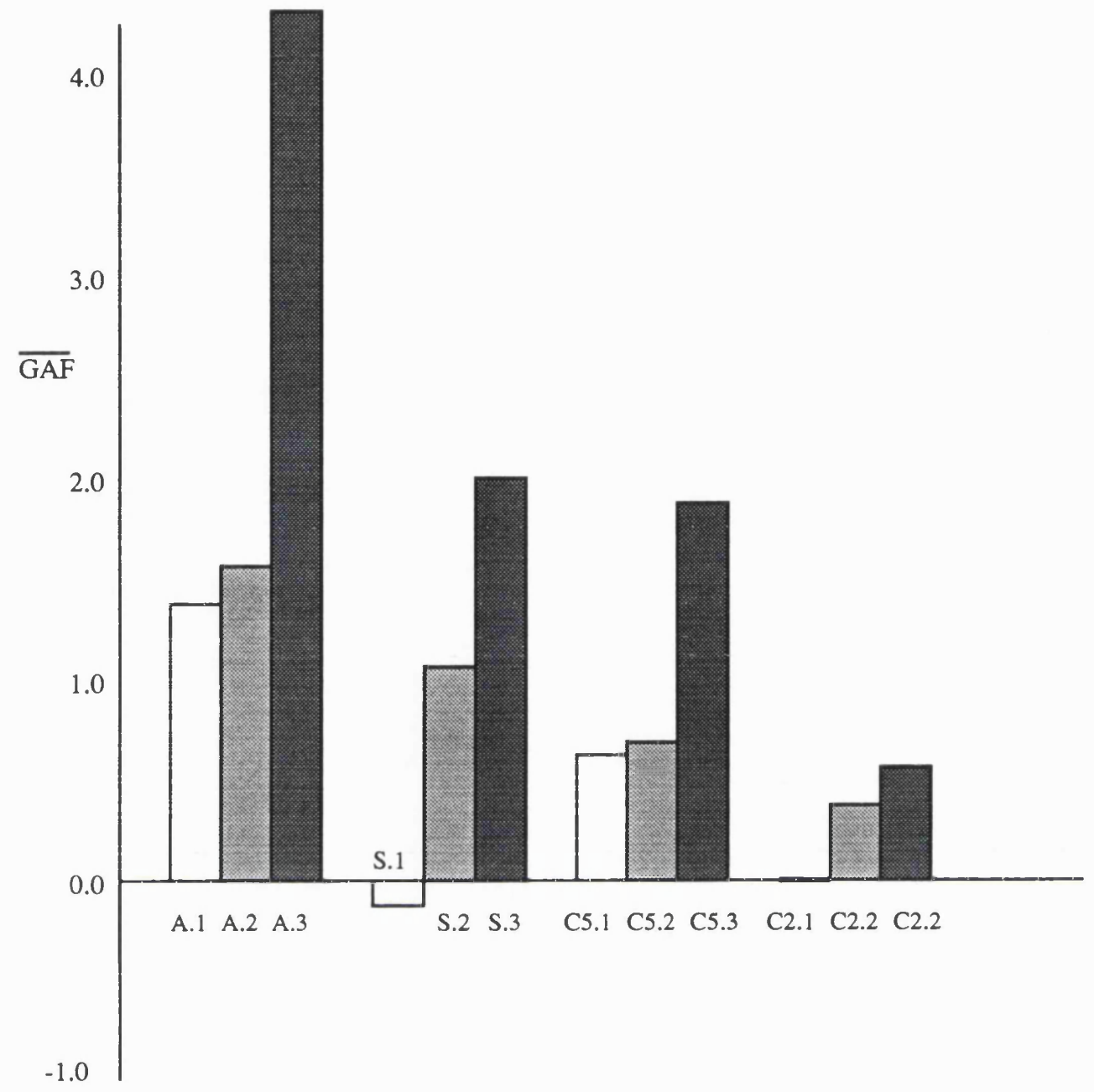

Figure 6.8: Comparative mean GAF achieved by UNIMEM, W-UNIMEM and CUNIMEM.

vised network (Network S.3) also demonstrates how previous links may be avoided with matching based on a contrast of common and distinctive features. However, this action is not necessarily an improvement. For example, in Network S.2 (Figure 5.3) Case 0910084 (SEDC) was correctly grouped with the two other cases of SEDC (091-0185 and 092-0181). However, in Network S.3 (Figure 6.7), Case 091-0084 remains unclassified. Node N8 in Network S.3 is initially created by a generalisation between the two cases of SEDC: Case 091-0185 and Case 092-0181 (this match achieves a similarity score of 22.7 and creates a concept node with 14 listed abnormalities). Both these cases are described in significantly greater detail than Case 091-0084 (all three cases of SEDC originate from different hospitals with different referring physicians). Case 091-0185 has 27 listed 
anomalies, whilst Case 092-0181 lists 32 dysmorphic features. Case 091-0084 lists only 10 abnormalities. This description does include epiphyseal abnormality of the long-bones, which is the relevant key hard abnormality. With W-UNIMEM, which bases evaluation on purely common features, this feature along with relevant secondary anomalies proved sufficient to group Case 091-0084 with the other instances of SEDC. However, with the C-UNIMEM algorithm, which utilises an asymmetrical evaluation contrast, when case 091-0084 is matched with Node N8 the similarity score only reaches $8.0\left(E_{t}=15.0\right)$ causing the case to remain uncategorised. The conclusion to be drawn from this occurrence is that whilst W-UNIMEM eased the problem of case non-uniformity by only operating with very significant, common abnormalities, the problem of non-uniform case descriptions is more evident with C-UNIMEM, which operates with all features of all levels of diagnostic significance, common and distinctive. Despite this factor, the overall performance of C-UNIMEM is greater than both UNIMEM and W-UNIMEM. Figure 6.8 shows the relative mean group accuracy factors for UNIMEM, W-UNIMEM and C-UNIMEM. The $\overline{G A F}$ for each concept network generated by C-UNIMEM is considerably higher than the corresponding $\overline{G A F}$ 's for both UNIMEM and W-UNIMEM. With the Acrocephalosyndactyly data set, C-UNIMEM achieves a $\overline{G A F}$ of +4.280 (Network A.3), and with the skeletal dysplasias data set the $\overline{G A F}$ for Network $\mathrm{S} .3$ is $\mathbf{+ 2 . 0 0}$.

The networks generated by C-UNIMEM with the cases with chromosomal abnormalities, C5.3 (Figure 6.9) and C2.3 (Figure 6.10), also have mean group accuracy factors that are greater than their respective predecessors. These networks also demonstrate the influence of the contrast model. They too display fewer extraneous concept nodes. In fact Network C5.3, which incorporates cases with a duplication in the number 5 chromosome, now only consists of correct groupings in terms of the associate banding defects. Also, it is interesting to note that no case is located at more than one node in either Network C5.3 or C2.3 (del(2) cases). Both Networks C5.3 and C2.3 were produced with the same parameter settings as those used for the Acrocephalosyndactyly cases (Tables 6.2 and 6.3). Whilst it would be possible to decrease the number of unclassified cases by altering the relevant system parameters, it is important to note that grouping as many cases as possible is not the real goal of the operation. If only a handful of cases are grouped, or classified, then this should not be seen as a failure. The research aspect of dysmorphology that constitutes conceptual learning, as demonstrated by the CBL model, is such that any new links that are established (between undiagnosed cases) through this 


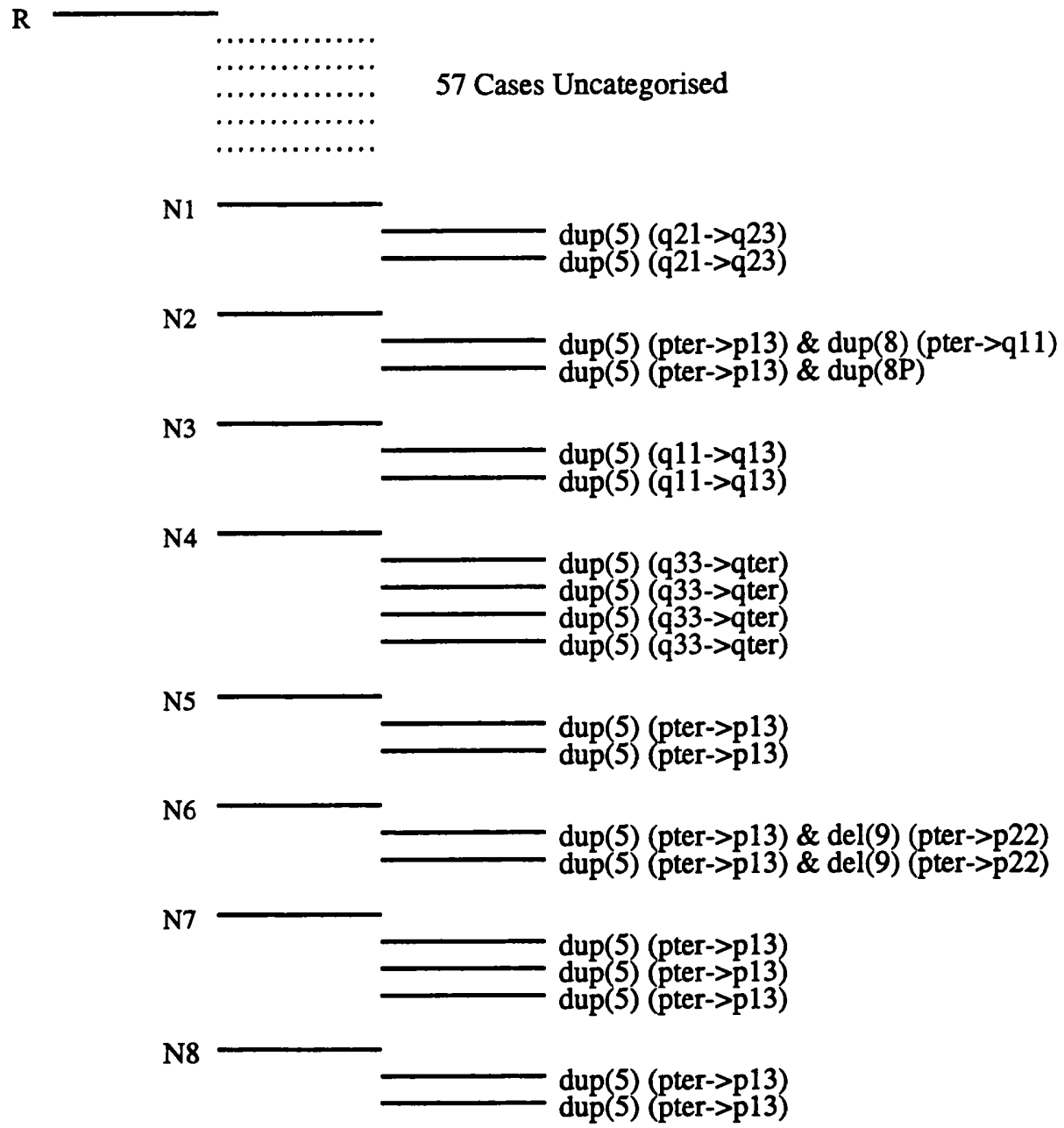

Figure 6.9: Network C5.3: Concept hierarchy generated by cases with a duplication in the number 5 chromosome.

type of analysis contribute to a new understanding of the domain. Furthermore, when creation of new concepts (or syndrome categories) is the main goal of operation, then this is with a view to using these concepts to solve problems. That is to say, the concepts should provide a useful prototype against which to diagnose future cases. Thus, the learning goal does have an explicit link with the diagnosis task. An important aspect of both the W-UNIMEM and C-UNIMEM models is that concepts are formed with a greater depth of category information than those of the UNIMEM model. That is to say, they store knowledge of relative feature salience within the concept description, which is in turn used for later assessments of similarity. The difference in performance with the non-chromosomal and chromosomal data sets is a reflection of the fact that the former data sets are a more representative collection of specific syndromes, whereas cases with 


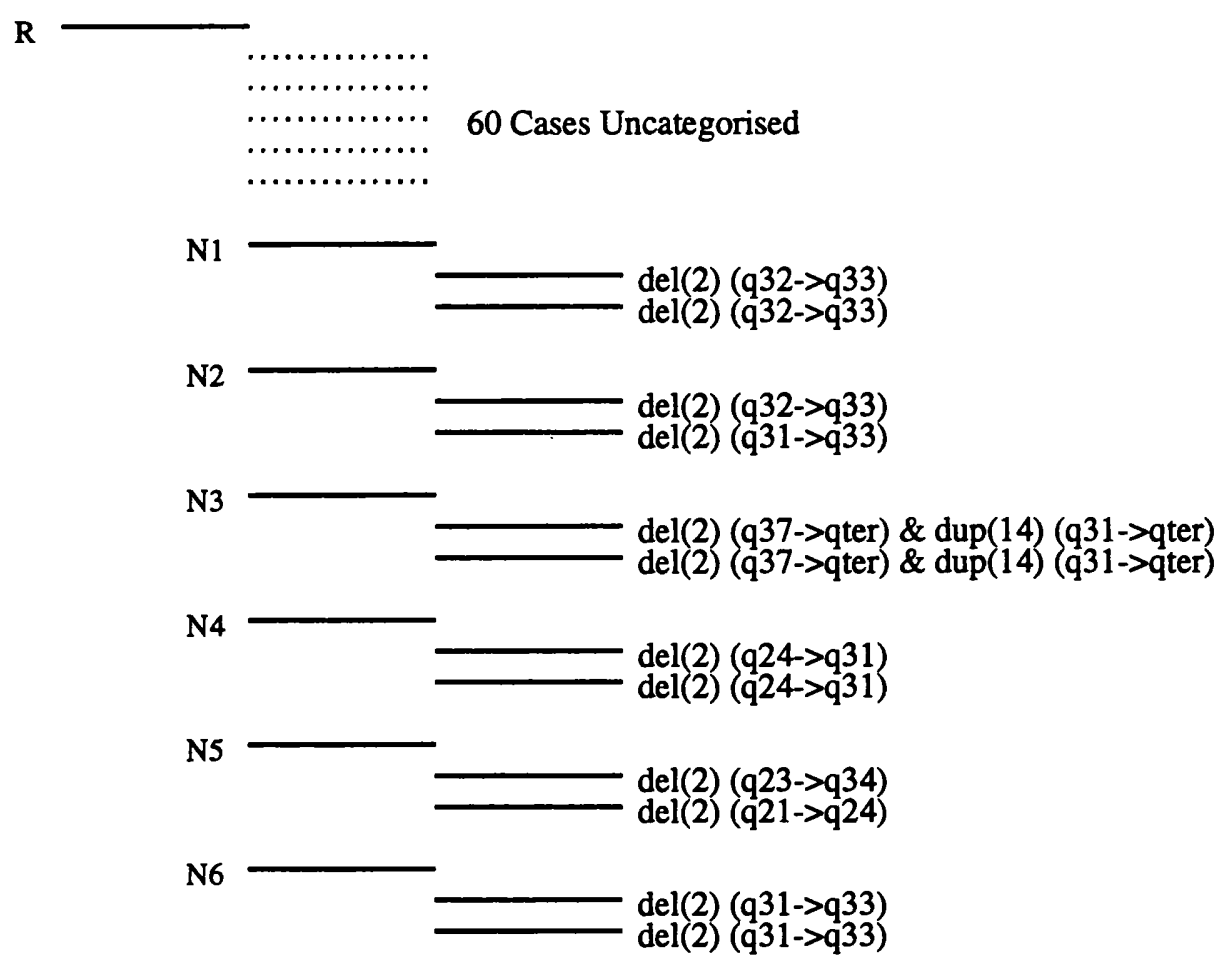

Figure 6.10: Network C2.3: Concept hierarchy generated by cases with a deletion in the number 2 chromosome.

chromosomal abnormalities (that are stored in HCDB) have no established prototype and suffer to a far greater extent from environmental noise.

The use of a contrast in matching results in a decrease in extraneous links between cases. A consequence of this is that more cases may remain unclassified, and the creation of sub-nodes through generalisation is less abundant. A facet of this type of (discriminative) algorithm is that the further a search proceeds down the hierarchy, the smaller the object description (case or node) that is encountered. However, the evaluation and generalisation thresholds remain constant. Thus, for sub-nodes to be formed there has to be sufficient weight in matches based on fewer features as search progresses downwards. In versions 1 and 2 of the algorithm, when matching was performed solely on common features, there was sufficient weight, in certain circumstances, for $G_{t}$ to be attained, resulting in the generation of child concept nodes. Now that distinctive features make a contribution in matching, the attainment of $G_{t}$ at sub-node levels is more difficult. To increase the depth of the network one could simply adjust the relevant system parameter. However, with the LDDB and SDR data sets, the parameter values listed above produce, 
in terms of depth and breadth, a close approximation to what is known to be correct.

\subsubsection{Discussion}

As with previous versions of the CBL program, the generation of alternative grouping patterns is achieved through manipulation of the system parameters. Again, if the data incorporated unknown (or undiagnosed) cases a specialist would be required in order to achieve sensible case groups. It was noted earlier that the incremental presentation of cases over a short time frame is not a true reflection of the real world. Thus, if the supervisor is an experienced specialist, then an experiment like those described above would, in this sense, provide a very artificial environment. With this fact in mind, some experiments involving incremental grouping of cases and concept formation have actually been performed with dysmorphology specialists. The main aim of these experiments was to assess the applicability of incremental concept formation, or case-based learning to dysmorphology. A tenet of this thesis is that the general theory of case-based reasoning and learning maps well with the actual processes of diagnosis and research, or learning, in dysmorphology. However, taking into account the nature of the available data (symbolic, or printed material), and the artificial time frame against which concept formation occurs in this instance, it was considered a useful exercise to test whether domain experts could successfully speed up these processes. The results of these experiments are reported at this point in the thesis in order to provide context for a critique of the CBL program and a comparison of its performance against human experts. Furthermore, the ensuing discussion provides an additional frame of reference for a general discussion of similarity assessment as an independent research topic with regard to CBR.

The experiments with dysmorphology specialists were performed using the SDR data set $^{2}$. During the tests, each case comprised a separate printed list of dysmorphic features (sub-categorised by the SDR skeletal components, see Table 4.5), without the listed diagnosis. The cases were presented incrementally to the experts, who were instructed to group the cases accordingly. The consultants were encouraged to explain why they grouped cases together (in terms of characteristic features) as they did so. When all the cases in the set had been presented, the experts were asked to describe the groups in terms of their prototypical abnormalities.

The first test was performed by two specialists in dysmorphology and clinical genetics,

\footnotetext{
${ }^{2}$ The consultants that participated in one of the tests actually compiled the LDDB data set.
} 
who, for the purposes of describing these experiments, will be referred to as Clinician $A$ and Clinician B. A second (identical) test was performed by one consultant who specialises in skeletal disorders, referred to as Clinician C. The experts were left very much to their own devices in these experiments. They were not told how many syndromes were accounted for in the data set. They were not specifically told to form a hierarchical network. The consultants were merely instructed to group the cases according to their interpretation of prototypical abnormalities. For consistency, and ease of comparison, the results from these tests are presented as concept networks (Figures 6.11 and 6.12) with associated node descriptions (Tables 6.2 and 6.3). However, it should be noted that there was no concept of a root node when groups were formed. Also, individual cases that would be regarded as unclassified with respect to the CBL program, effectively form their own group in this instance. Comments on why these cases were not linked with others is noted in the relevant description.

An important point to note is that the experts involved in these tests specialise in different aspects of dysmorphology. Clinicians A and B are experts in clinical dysmorphology, whereas Clinician C is a specialist in skeletal disorders. Therefore, the first two physicians examine human patients whilst the third consultant examines $x$-rays. Neither, in the course of examination and diagnosis, would analyse purely symbolic data such as printed medical records. An interesting result from these experiments is that, in terms of the respective group accuracy factors, the clinical dysmorphologists have performed slightly better than the radiology expert, despite the fact that the test data was across known skeletal dysplasias. This serves to emphasise the artificial nature of these experiments.

The relative performance of the human experts compared to the three CBL programs is represented graphically by Figure 6.13. This chart shows the mean group accuracy factors for the concept networks created in the tests with the respective physicians (W.1 and H.1), along with the $\overline{G A F}$ 's calculated for the networks generated by UNIMEM, WUNIMEM and C-UNIMEM with the skeletal dysplasias data set. Network W.1, created by Clinicians $\mathrm{A}$ and $\mathrm{B}$, has a $\overline{G A F}$ of +1.17 . This corresponds to a better mean group accuracy factor than the equivalent networks generated by UNIMEM and W-UNIMEM (although only a slight improvement over Network S.2 which has a $\overline{G A F}$ of +1.025 ). This score falls short, however, of the $\overline{G A F}$ managed by C-UNIMEM $(+2.00)$. Network H.1, created by Clinician $\mathrm{C}$, has a $\overline{G A F}$ of -1.65 . Thus, not only is the mean group accuracy 


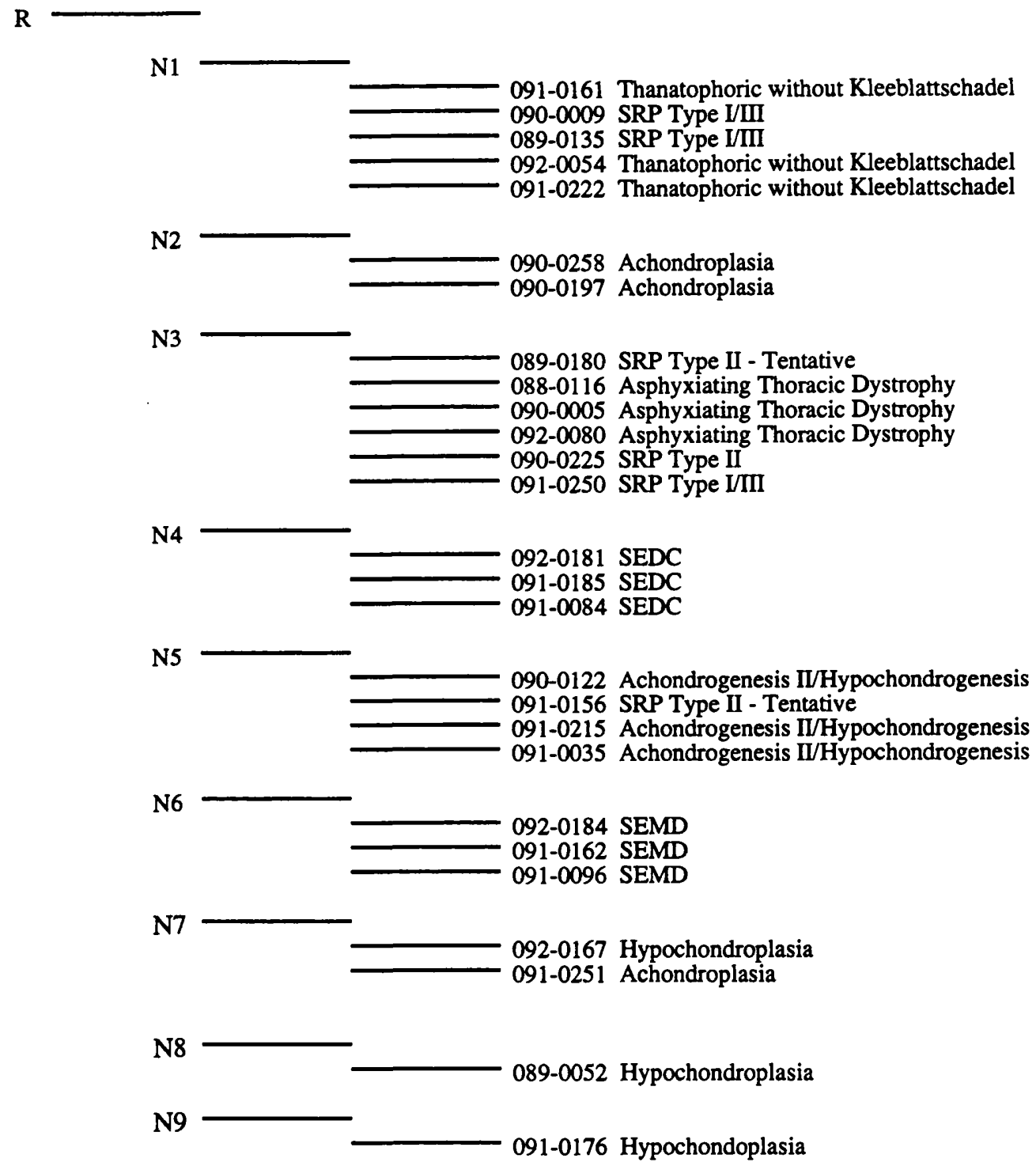

Figure 6.11: Network W.1: Concept nodes formed by Clinician A and Clinician B with SDR cases. 


\begin{tabular}{|c|c|}
\hline Node & Description \& Comments \\
\hline N1 & $\begin{array}{l}\text { Flattened vertebral bodies, } \\
\text { severe early problems (i.e., neonatal deaths/stillborns/abortions), } \\
\text { short limbs, severe to moderate rib shortening, } \\
\text { and pelvis trident notched. }\end{array}$ \\
\hline N2 & $\begin{array}{l}\text { Mild rib shortening, flattened spine, } \\
\text { and flattened acetabular roof. }\end{array}$ \\
\hline N3 & $\begin{array}{l}\text { Relatively normal spines but very short ribs. } \\
\text { Didn't compare pelvis, but indicated that this should have been done. } \\
\text { Noted that all cases except } 089-0180 \text { had (pelvis acetabular-roof trident). } \\
\text { It was noted that cases } 089-0190 \text { and } 090-0250 \text { had craniosynostosis } \\
\text { and polydactyly. }\end{array}$ \\
\hline N4 & $\begin{array}{l}\text { Relatively early onset, relatively normal rib size, } \\
\text { bell shaped thorax, short limbs, } \\
\text { unossified pubis, and absent talus. }\end{array}$ \\
\hline N5 & $\begin{array}{l}\text { Early onset, poor or unossification of the spine, } \\
\text { short limbs, and barrel shaped chest. } \\
\text { It was noted that there is similarity with group N4. } \\
\text { The consultants were unsure about case } 090-0122 \text {. }\end{array}$ \\
\hline$\overline{\text { N6 }}$ & $\begin{array}{l}\text { All cases are alive and have } \\
\text { epiphyseal/spondyloepiphyseal abnormalities. }\end{array}$ \\
\hline N7 & $\begin{array}{l}\text { Some spinal abnormalities. } \\
\text { Appeared to be (spondylo)metaphyseal problems. } \\
\text { Required more information on epiphysis and status (alive or dead). }\end{array}$ \\
\hline N8 & $\begin{array}{l}\text { Noted as difficult to group. } \\
\text { Age and sex were noted - } 15 \text { year old male. } \\
\text { Slightly short bones. } \\
\text { No details on epiphyseal or metaphyseal defects. }\end{array}$ \\
\hline N9 & $\begin{array}{l}\text { Again difficult to group. } \\
\text { Age and sex noted - } 18 \text { year old female. } \\
\text { Normal hands, but feet unknown. } \\
\text { The consultants indicated that cases } 091-0176 \text { and } 089-0052 \\
\text { required more detail. It was implied that they may be comparable } \\
\text { however the fact that case } 091-0176 \text { has normal hands prevented this. }\end{array}$ \\
\hline
\end{tabular}

Table 6.2: Clinicians A and B group descriptions of SDR cases.

achieved by this physician (a radiology specialist) less than that achieved by the clinical geneticists, but it also falls considerably short of the scores achieved by the three CBL programs. A major contributor to this phenomenon is the Node N4 in the respective concept Network H.1. This node groups seven cases whose diagnoses range across all three syndrome families that exist in the SDR data set. Thus, the negative contribution to the group accuracy factor for Node N4 is considerable (if a separate group had been formed with the three cases of Thanatophoric dysplasia the resulting $\overline{G A F}$ would have been -0.107).

The node descriptions given by the physicians reiterate that syndromes are distinguished by a pattern of anomalies. Each group of cases is described in terms of a loose association, or conjunction, of abnormalities with respect to the skeletal components. 


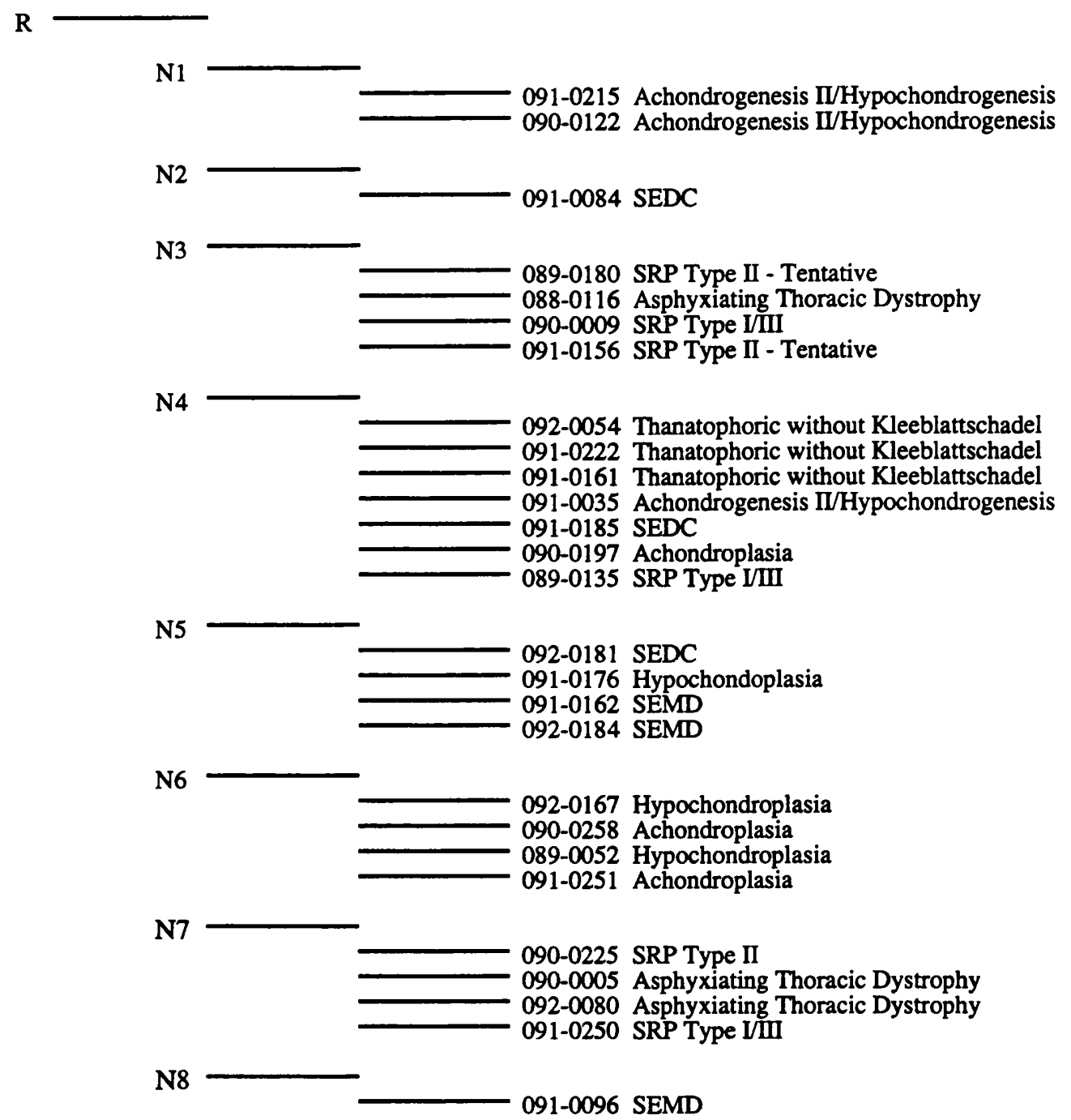

Figure 6.12: Network H.1: Concept nodes formed by Clinician C with SDR cases.

However, a couple of group descriptions contain a relationship between a normal and abnormal feature. For example, the description of Node N3 in Table 6.2 lists "relatively normal spines but very short ribs". It is the combination of these two factors that is regarded as a distinguishing feature of the group, rather than one specific dysmorphic anomaly. A feature can, therefore, refer to two inter-dependent abnormalities, or an observed combination of two anomalies, one of which could be a normal clinical or skeletal region. Normality is noted on several occasions. For instance, in Node N2 of Table 6.3, normal long-bone size is emphasised. References to normal hands are evident in both description tables. When normal regions are noted, it is usually with reference to the overall group pattern, i.e., normality is generally described in terms of other abnormal 


\begin{tabular}{|l|l|}
\hline Node & Description \& Comments \\
\hline N1 & $\begin{array}{l}\text { Both cases a fetus. } \\
\text { Short limbs, absent ossification of the spine, } \\
\text { and iliac abnormality (arched). }\end{array}$ \\
\hline N3 & $\begin{array}{l}\text { Normal long-bone size (noted as unusual in bone dysplasias), } \\
\text { rib width is thin. }\end{array}$ \\
\hline N3 & $\begin{array}{l}\text { Three are neonatal deaths (1 unknown). } \\
\text { All have severe rib shortening, limb shortening } \\
\text { and abnormal pelvis. } \\
\text { It was noted that case 088-0116 had a poor level of detail. }\end{array}$ \\
\hline N4 & $\begin{array}{l}\text { All stillborn or neonatal deaths. } \\
\text { Short limbs, flattened vertebral bodies, abnormal pelvis } \\
\text { and all have moderate rib shortening. }\end{array}$ \\
\hline N5 & $\begin{array}{l}\text { All alive. } \\
\text { All short limbs, abnormal spine, abnormal pelvis and } \\
\text { reasonably normal hands. } \\
\text { It was noted that case 092-0184 was probably in this group } \\
\text { i.e., slightly tentative about this case. }\end{array}$ \\
\hline Nwo cases are alive and two unknown. \\
All have short limbs. \\
Abnormal pelvis (flat acetabular roof) except for case 089-0052, \\
which has a normal pelvis. \\
All have abnormal hands.
\end{tabular}

Table 6.3: Clinician C group descriptions of SDR cases.

features. Normal clinical or skeletal components are not regarded as independent features in the same sense as dysmorphic abnormalities. They are relative, or contextual in nature.

Another characteristic of the group descriptions is the frequent use of generalisation with regard to individual features. The symbolic format of dysmorphic features has three levels: the skeletal region, an attribute, and a specific anomaly. Some node descriptions list distinguishing features that comprise a generalisation of the two highest levels (region, attribute), which are noted as abnormal, without a specific anomaly (e.g., abnormal spine). Furthermore, these generalisations are not constrained to include all the grouped cases. For example, the phrases "relatively normal rib size" (Node N4, Table 6.2), and "reasonably normal hands" (Node N5, Table 6.3) imply a feature exhibited by the group in general, rather than each individual case. This concurs with the polymorphic facet of syndrome descriptions.

An aspect of the domain that stands out from these results is the importance of 
age and status with regard to presented cases. The consultants clearly referred to these details, and attributed high diagnostic value to this information. Unfortunately, the SDR group is the only data set of the four used in this research that includes information about the age and sex of the infant (even so, this information is still not complete). With respect to the SDR cases, there appears to be a clear distinction between patients that belong to a 'surviving' syndrome, and cases of 'early onset'. The experts consistently divided cases

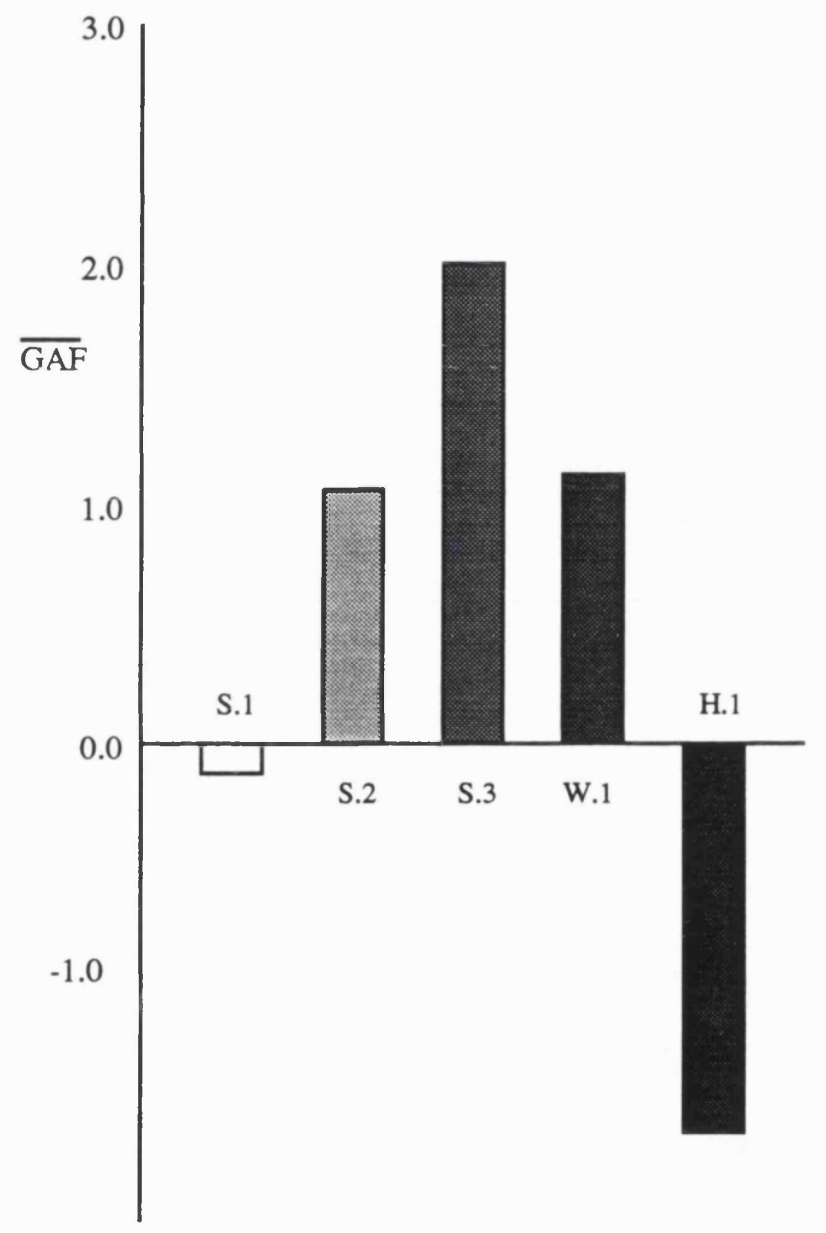

Figure 6.13: Mean GAF for Networks S.1, S.2, S.3, W.1 and H.1

with respect to this distinction. Following on from this, although the SDR set provides by far the most comprehensive case descriptions of the four data sets, even this group suffers from inconsistency, or non-uniformity. This seems particularly relevant to the cases of Hypochondroplasia. Network W.1 (Figure 6.11) shows that two of these cases stand isolated, and the associated descriptions note that Clinicians A and B commented on the lack of information for cases in groups N7, N8 and N9 (in particular, two cases of 
Hypochondroplasia listed a surviving age, but this information was missing for the third case). Whilst Clinician C did link together two cases of Hypochondroplasia, along with two cases of Achondroplasia (group N6, Figure 6.12), it was also noted that for two cases in this group the age and status details were unknown.

The reluctance of domain experts to define mutually exclusive syndromes that form category hierarchies has already been emphasised in this report. Thus, when these experiments were performed, the experts were not asked to form hierarchies, and there was no constraint determining that a case could only be linked to one group. However, both these aspects of categorisation can be interpreted from the group descriptions offered by the experts. For instance, Clinician $\mathrm{C}$ notes that Case 091-0096 could be linked to either

\section{Addendum}

It should be emphasised that the discrepancy in the results of the grouping experiments performed by the different clinicians will almost definitely reflect a difference in their approach to the task and the manner in which they work, as well as their interpretation of diagnostic value with regard to recorded patient features. The artificial nature of these experiments do not reflect true clinical practice, but are intended to demonstrate a case-based reasoning approach to diagnosis and learning. The clinicians generously co-operated with this task in mind.

ucucascs we sigumicance of uraering on tne resutant network. Another related issue is that when such re-appraisal is performed, each individual case is assessed in terms of its complete list of dysmorphic features, not just those that are distinctive from the group prototype.

As a general summary to this section, the results from the experiments involving dysmorphology specialists demonstrate that case-based learning is feasible with the available data. This conclusion can be drawn from the fact that, overall, the experts managed to form sensible groups (with respect to the known diagnoses) and establish corresponding prototypical concept descriptions. Of course, the experts have much specific medical knowledge and experience that is not readily available to the CBL programs. The simplistic diagnostic model that is available, in conjunction with the contrast model of similarity 
assessment does, however, produce accurate case groups with respect to the known diagnoses, particularly with the LDDB and SDR data sets (which comprise representative patient records). The following section summarises the merits and weaknesses of the CBL model that may be concluded from the experiments described in this, and the previous two chapters.

\subsection{A Critique of the CBL Model}

A key principle of the CBL model is that the case memory is organised into a hierarchy of increasing specificity. With this axiom in place, confined search in conjunction with a suitable matching operator locates similar entities in the case memory and facilitates reorganisation of category information. In the context of a localised group of cases, such as those used in the reported work of this and the previous two chapters, then such a scheme is acceptable.

A primary concern of specialists is that syndromes are not mutually exclusive. This is not a problem provided the relevant algorithm develops a graph network, not a tree, and concepts are polymorphic (i.e., a stored instance, or case, is not required to have a conjunction of all the prototypical features listed by the concept). The UNIMEM model satisfies both these criteria. However, UNIMEM (and the subsequent CBL programs that are based on it) operates in a discriminative manner. Thus, individual cases are only stored in terms of their discriminating features. This scheme complements a hierarchical, top-down search algorithm. The algorithm and case representation are, in this instance, inter-dependent. However, it has been demonstrated that such a scheme has inherent problems with respect to dysmorphology. Whilst hierarchies do exist in terms of the syndrome nomenclature, they are not rigid and are not interpreted strictly by physicians. When matching a new case to the known syndrome nomenclature, a physician would take into account both the relevant syndrome prototype and specific individually recorded patients. Such cases would naturally be documented in terms of all evident features (it is possible that normal regions may also be documented). Finding such cases would not necessarily be a top-down search procedure, and matching would certainly take into account the complete malformation pattern of each subject. Thus, taken in this broader context, the hierarchical architecture and associated mechanisms of the CBL model are restrictive. 
This architecture presents other related problems. A facet of the discriminative approach is that the order of presentation of cases has a direct effect on the resulting case network. Individual cases can become hidden by the higher order prototypes below which they are stored. It is unclear what effect ordering might have on actual syndromes and documented cases, but the shallow nature of syndrome nomenclature, and the fact that cases are recorded in terms of all relevant features, would limit this problem. If cases are initially diagnosed incorrectly, then this is generally with reference to a syndrome family (i.e., to the wrong variation of related syndromes). Further information (perhaps at a later date) may facilitate a correction in this instance. This kind of procedure is very similar to the localised learning that occurs when new variations of a known syndrome family are discovered (through reorganisation within the group). This type of ordering problem is different to that displayed by the CBL program. The CBL organisation actually prevents the retrospective assessment of individual cases that was demonstrated by the tests with human experts. According to Lebowitz, over time and with a large data set, the effect of case ordering is not strong [50]. This statistical argument may hold for domains in which large amounts of statistical data exist. In dysmorphology, however, this is not normally the case, and a syndrome definition can be based on only a few individual cases. Whilst this research has not put great emphasis on case ordering, due to the non-conformity of the dysmorphology field (in terms of the availability of large and comprehensive data sets) with the assumption noted above, it was considered that the question of ordering should not be overlooked. Appendix $\mathrm{C}$ describes some additional tests performed with the C-UNIMEM algorithm using the Acrocephalosyndactyly data set. A general conclusion that is drawn from these tests is that the algorithm performs consistently in terms of grouping accuracy. However, ordering does appear to have a lower level effect with regard to node descriptions due mainly to the problem with the discrimination approach outlined above, and the fact that once a node description is constructed, the cases subsequently stored beneath it (and their case descriptions) are not given further consideration with regard to that description.

Another, related, problem is that the dysmorphology domain is inherently incomplete and noisy. Case and syndrome descriptions suffer from non-uniformity and personal bias. Such variation in detail with respect to documented cases has also been shown to have a detrimental influence on system performance. This problem is accentuated in UNIMEM which employs a similarity metric that has a direct dependency on the size of 
a case description. The modified versions of the algorithm incorporate individual feature salience and consequently suffer less in the presence of non-uniform data.

It has been indicated that similarity assessment is crucial to the performance tasks of the domain. Furthermore, the contrast model that is employed by the C-UNIMEM program is seen as a compromise solution. It is certainly difficult to foresee a mathematical model that will cope totally with the incompleteness and complexity of a medical domain such as dysmorphology. However, the contrast model does offer some basic axioms that lend themselves well to the different perspectives of similarity demonstrated in dysmorphology. The only requirement that the contrast model has, in terms of domain knowledge, is a model of feature salience. Fortunately, such knowledge is readily available through LDDB's encoding of dysmorphic feature weights (although strictly speaking this represents a close approximation to diagnostic feature salience). Analysis has shown, however, that similarity involves a number of factors that have not been accounted for. Age and status information is not available in the majority of cases. The concept of 'normality' is not used, although what defines normality is a contentious point. It has become apparent through the tests with the consultants that unless a clinical region has a specific abnormality recorded then it is generally assumed to be normal. The SDR data set specifically lists some skeletal regions as normal, some as unknown, and for some there is no indication of whether it is unknown or normal. The experts were subsequently unsure whether or not to assume that certain regions were normal. A generalised condition that combines an abnormal feature to a normal region within a group description (as seen in in the tests with domain experts) is not facilitated in the CBL model (a feature of the UNIMEM system is that concepts are formed in terms of a conjunction of features; it does not cater for disjoint conditions).

Another aspect of matching demonstrated by the human experts is that individual abnormalities can be generalised when viewed in the context of a case group. That is to say, in order to describe a prototypical malformation pattern, it is sometimes useful to record the abnormal clinical region that is common to the relevant cases rather than a list of specific anomalies. This may happen if the physician sees enough evidence to suggest that the anatomical component is generally abnormal (if there was a hard, or very significant abnormality then this type of generalisation is less likely). Generalisations of another type are also made due to the non-uniform nature of patient records. One physician may describe a feature as "mild", whereas another specialist, in a separate 
situation (and with a different patient) may record the same feature as "moderate". This type of interpretation can be abstracted by a third expert with both case descriptions at hand in order to infer an equivalence between the two. The matching performed by the three CBL programs cannot cope with this situation as it is symbolically exact according to the three level encoding of dysmorphic features. The LDDB system (which was designed by experts), however, specifically allows loose matching (albeit for the purpose of indexing) in order to account for this type of user interpretation.

A natural progression of the CBL model (in terms of the three different versions) has been an increase in the supervision of the algorithm through system parameters. The final version, which incorporates the contrast model, allows the most flexible supervision in the sense that bias may be attributed to the common or distinctive features depending on the goal of the match. However, the user cannot vary the weight of specific abnormalities. Emphasis is controlled only in terms of common or distinctive sets. It has become apparent that experts can focus very specifically on one or two very significant features, and virtually discard other anomalies as environmental noise. Such a situation would

\section{Addendum}

The underlying model used within similarity assessment was based on phenotypic features and their associated level of diagnostic significance. This model assumes, therefore, that no conclusive genetic linkage exists for the patient (i.e., either the karyotype was inconclusive or not performed). It should be stressed that diagnosis will normally commence with a karyotype. If sufficient genetic information was available, this could have a great effect on the procedures involved with similarity assessment. Thus, the inclusion of a comprehensive genetic model within this work could result in an alternative model of similarity assessment.

required to exhibit a feature before it is made permanent, or prototypical. However, the nature of the domain is such that many syndromes have only a few known cases. In this situation the concept of prototypicality is less certain. The conclusion has already been made that if the system is to be supervised, then the users involvement could broaden in order to oversee case-base reorganisation along with the other key processes of CBR. 


\subsection{CBR and General Similarity Assessment}

At the second DARPA case-based reasoning workshop [39] discussion was partitioned across issues considered to be central to CBR. Along with topics such as case representation and indexing, a panel was delegated to appraise similarity assessment in the context of CBR [10]. The output from this discussion group was a list of seven questions that summarise the open issues of similarity assessment in CBR. This section is intended to provide answers to these questions in the light of the work reported in this thesis that concerns similarity assessment specific to dysmorphology.

1. Why is one situation perceived as being similar to another? This vague question is intended to summarise the essence of the discussion. The main issue is concerned with whether or not general principles for similarity assessment may be established with respect to a designated domain. In dysmorphology, the general principle that has been established is that similarity is viewed as a pattern of malformations of varying diagnosticity.

2. What is the relationship of case retrieval and similarity assessment? A distinction can be made between similarity assessment that operates in conjunction with case retrieval, and general similarity assessment which can constitute an independent process. The former is dependent on the structure of the database and the associated complexity of categorisation within the case memory. This determines whether or not effective case retrieval may be performed by simple indexing (a boolean conjunction of features), or requires a more careful evaluation of common and distinctive attributes. The flat database structure of LDDB represents the first instance where boolean indexing will suffice if guided by an expert and no ranking of database entities is required. The CBL model employs a database structure organised by category information against which case retrieval has the goal of finding the best, or most applicable locations in case memory. Thus, a ranking of candidate syndromes is implicit within the similarity assessment that is performed with search (or case retrieval).

The second type of similarity assessment will operate once the relevant hypotheses have been retrieved. In LDDB, this assessment is left to the human expert. In ranking systems, such as SYNDROC, this type of assessment is facilitated by 
mathematical formulae in order to refine competing hypotheses. In the CBL model a generalisation function determines whether a new case should merely be stored below a retrieved category, or whether sufficient similarity exists with a past case to form a new category. The CBL system actually employs the same model to perform both types of similarity assessment. The contrast model can be used in this way because it allows the emphasis of the match to be altered though its parameters.

3. Can cases be represented as sets of "simple" features? The initial answer to this question is "yes". The fact that a number of computer databases have been developed that employ a simple attribute-value representation for both syndromes and cases confirms this. However, should the question really be interpreted as "how much inferential effort should be concentrated in representing cases as opposed to problem solving procedures?" The issue is then one of finding the most suitable balance between the knowledge stored within the case representation, and that stored within program functions or the domain knowledge-bases that they access. In dysmorphology, this relates to the performance goals of the system along with the constraints of the project (as discussed in Chapter 2). In LDDB, a simple encoding does provide the case representation. Intelligent processing of cases is facilitated by a totally separate model of domain knowledge, which in this case is a human expert. In this instance, the relationship between the knowledge encoded within the case representation and the knowledge required for processing is deliberately unbalanced. In SDD, this balance is more equal. The diagnosticity of individual dysmorphic features is encoded within syndrome frames (which may be regarded as prototype cases). This representation facilitates a more informed retrieval phase. Having retrieved relevant candidates, SDD then employs several independent, but domain dependent, knowledge-bases to promote the best hypothesis. Thus, SDD shares its domain knowledge between the case representation and independent models of expert knowledge. However, whereas with LDDB one can say that complete domain knowledge is shared across these two bodies (the database and the human expert), whether or not SDD satisfies this condition will depend on the success of the knowledge engineering used to elicit relevant expertise. In this respect, SDD has the favourable goal of being dedicated to a specific group of syndromes. This makes the elicitation of domain knowledge a feasible objective. With respect to 
the broader spectrum of syndromes, it is unlikely that such knowledge elicitation is possible without a considerable phase of knowledge acquisition. The CBL model employs a simple case representation and separate models for feature diagnosticity and similarity assessment. The feature significance levels constitute an independent domain knowledge model which, as discussed above, falls short of the depth of information stored by SDD. However, this domain knowledge is readily available without the need for knowledge elicitation. The contrast model does not provide domain knowledge, but again, it is readily available, and for the reasons argued at the beginning of this chapter, can be applied to dysmorphology.

4. How is similarity assessed when cases are not uniformly described? This issue is not unrelated to the above question concerning case representation. Researchers view the solution to this issue as a choice between engineering a uniform representation and deriving an inference mechanism that can account for non-uniformity. Work to date has been shared across both these options. Most dedicated computer systems have tried to standardise the case representation with respect to dysmorphic feature nomenclature (e.g., the POSSUM system which includes images in order to ease the problem of uniformity). With no inference mechanisms in place, the user is usually required to assess the equivalence of feature descriptions. LDDB and SDD devote varying degrees of effort to inference mechanisms. The loose coupling facility of LDDB can account for the variance in feature descriptions (any inference as such is made by the expert). However, case descriptions can also vary in size. The diagnostic feature weights provided for use with LDDB (and used by the CBL model) ease this problem to an extent. SDD on the other hand, along with the diagnostic information encoded within syndrome frames and its background knowledge-base, has separate knowledge of linguistic feature equivalences (mild verses moderate etc.,) which can be accessed during the diagnostic cycle.

5. What is the role of general domain knowledge in similarity assessment? Domain knowledge is critical in dysmorphology. Dysmorphologists are specialists that have knowledge and experience specific to diagnosing syndromes, but underlying this have a general medical foundation without which the necessary expertise could not be acquired. In terms of a computer model, the requirement for such 
general medical knowledge is, again, dependent on the system goals. The contrast between LDDB and SDD demonstrate this, i.e., if the system is to perform unsupervised, comprehensively and intelligently, then such knowledge will be required.

6. How is similarity assessment influenced by problem solving contexts? In terms of the two styles of case-based reasoning, dysmorphology provides a domain for classification CBR. A problem solving context can be interpreted, however, when diagnosis (and learning) is performed within the context of a family, or group of like disorders. This research has employed the contrast model partly to account for this phenomenon. The significance of individual anomalies can vary depending on whether they are common or distinctive, and on the directionality of the match. Thus, in this sense, the context of the problem (diagnosis or learning) does influence the similarity assessment.

7. Can similarity be computed or is it simply known from past experiences? In dysmorphology, this can be a combination of both. The level of experience of the consultant is the key. Inexperienced physicians will rely more on relevant literature, syndrome compendia and computer databases to retrieve candidate hypotheses. This involves assessing the similarity of the patient to recorded syndromes, and is therefore a form of computation. More experienced specialists can sometimes skip this type of computation. In this sense, similarity assessment can be viewed more as experience than computation. If gestalt recognition promotes one hypothesis, similarity is then computed in order to confirm or reject the hypothesis. If there is a differential diagnosis, similarity is computed in order to derive the most likely hypothesis.

\subsection{Conclusions}

This and the previous two chapters have reported work specific to case-based learning in dysmorphology. A CBL model has been developed based on an algorithm taken from the literature. Two models have been used to enhance the system: a domain model, which distinguishes the salience of individual abnormalities, and the contrast model, a theoretical model of similarity which has characteristics that are particularly suitable to the domain. These extensions have resulted in two new versions of the UNIMEM 
program respectively: W-UNIMEM, which employs a model of diagnostic significance, and C-UNIMEM which employs both the the weighting model and the contrast model of similarity. Thus, the program has been augmented chiefly with regard to the functions that perform similarity assessment. The relative performance of these three programs has been examined with respect to the mean group accuracy factor $(\overline{G A F})$, a measure which reflects the accuracy of case clusters in terms of their known diagnoses. A clear improvement can be seen with regard to this measure with each successive version of the CBL model. Furthermore, the resulting $\overline{G A F}$ achieved by C-UNIMEM with the skeletal dysplasias data set compares favourably with the equivalent scores derived from concept networks generated by human experts. The artificial nature of the experiments performed by the physicians should be noted. However, it should also be noted that purely symbolic databases are employed by human experts to assist their investigations, particularly with regard to diagnosis. The higher order theme of the work reported in this thesis has been that a symbolic learning program may also provide assistance to an expert user, although in this case, explicitly with regard to analysis of syndrome categories.

Whilst learning in dysmorphology has provided the theme for the development of a CBR 'assistant', such an algorithm can also be used to assist the primary dysmorphology task of diagnosis. The CBL model can, by default, retrieve potential hypothesis (i.e., syndrome objects in the form of prototypical concept nodes) through a combination of confined search and similarity assessment. Thus, the CBL model provides a general CBR architecture that facilitates both (syndrome) category reorganisation, i.e., learning, and (differential) diagnosis.

The CBL model is not ideal, and its main shortcomings have been highlighted. This critique should be viewed in the light of the thesis aims, which are to automate the learning aspects of the domain. With this aim, the work explicitly addresses a task not catered for by previous computer applications in dysmorphology. However, another objective is to also provide a practical dysmorphology computer system. That is to say, along with automated learning the system should, by default, assist the primary task of diagnosis. For this reason some of the unsuitable characteristics of the CBL model discussed above take on additional relevance. With these objectives in mind, the following chapter presents a general CBR assistant model which encompasses the work described in Chapters 3 through 6 whilst taking the practical issues involved with developing a useful CBR dysmorphology system into consideration. 


\subsection{Summary}

- The similarity functions of W-UNIMEM have been extended further by incorporating the contrast model of similarity assessment. This model allows similarity to be calculated in terms of both common and distinctive feature sets, and allows the 'bias' of the matching process to be varied in two ways: according to the directionality of the match, and according to the context of the match. In order to account for context the contrast model has been extended slightly from its original definition.

- Asymmetrical matching corresponds to the evaluation functions which implicitly form a diagnostic comparison (i.e., a case is compared to a prototype concept node). This asymmetry is modelled through the relative setting of the parameter $\theta$ with respect to the parameters $\alpha$ and $\beta$. The former is associated with common features, and the latter two parameters vary the contribution of distinctive features to the overall similarity. Common features are, thus, attributed additional weight in asymmetrical comparisons, i.e., evaluation. Also, contextual bias is given to common features when the task is evaluation (through the salience function $f_{i}$ ). Alternatively, when the task is implicitly learning, i.e., generalisation, the various parameters are set to reflect a more balanced contribution between the common and distinctive features of two cases.

- The performance of the C-UNIMEM program, which incorporates the contrast model, is a clear improvement over both UNIMEM and W-UNIMEM in terms of the mean group accuracy factor $(\overline{G A F})$.

- Additional experiments conducted with human experts demonstrate that symbolic incremental concept formation is feasible within dysmorphology. Furthermore, the comparative accuracy of C-UNIMEM is favourable with respect to those achieved by the human experts. These experiments highlight a number of limitations with regard to the similarity model. It should be noted, however, that the utilisation of a similarity 'formula' such as the extended contrast model is viewed as a compromise with respect to the constraints of the project. The ultimate goal of the CBR system is to 'assist' rather than comprehensively model the processes performed by human specialists. 
- The work reported on case-based learning, and specifically with regard to similarity processes in dysmorphology, has allowed some insight regarding the open issues of similarity assessment defined by the CBR community. 


\section{Chapter 7}

\section{A CBR Assistant for Diagnosis and Analysis of Dysmorphic Syndromes}

\subsection{Introduction}

This chapter presents a general CBR model which encompasses the work described in Chapters 3 through 6 . Chapter 3 described a case-based architecture which provided a platform on which to develop programs for two procedural tasks: retrieval of similar entities (syndrome or case records) and (syndrome) category reorganisation. The first procedure enables confined search within the case memory in order to retrieve both syndrome and case records that match a specified set of dysmorphic features. This program constructs a differential diagnosis and is also described in Chapter 3. The second task uses a case-based learning algorithm to operate with stored cases (and syndromes) for the purpose of establishing links between patients and creating associated (generalised) concept descriptions. This procedure promotes an analysis or research task with regard to the possible recognition of new syndromes. Chapters 4 through 6 are specific to this aspect of the thesis. Both these procedures are forms of case-based reasoning. Both employ object retrieval in conjunction with a similarity assessment phase. The main distinction between the two is that a case-based learning procedure also utilises its similarity assessment module with regard to automatic reorganisation of the case-base.

By incorporating these two procedural functions within its structure, the case-based 
reasoning system described in this chapter can assist a domain expert with the two principal tasks of dysmorphology. That is to say, the CBR system can assist the physician in retrieving and constructing a differential diagnosis, which in turn may lead to a firm diagnosis; and the CBL program that operates within the general CBR model can assist in analysing potential reorganisations of the syndrome nomenclature. An important theme of this chapter is that the general CBR model is very much an 'assistant' rather than a fully automated, artificially intelligent solution. This theme follows from the thesis goal of providing a realistic, or practical, CBR model for dysmorphology. With this in mind, the CBR model described in this chapter comprises some additional, but important, aspects to its design.

One feature of the general CBR system is that it is very much an interactive model. That is to say, it is designed with an acceptance that crucial decisions must be made by the human expert. This facet of system design is particularly important if the system domain theory is weak such as it is with dysmorphology. Kolodner refers to this type of model as 'case-based decision aiding' [44]. The CBR system performs specific functions within the overall CBR procedure, either to assist in selecting the most applicable precedents, or perhaps to suggest possible solutions, but important intermediate and final decisions are left to the human expert. As the system design becomes more interactive, or more of an assistant than a solution, then the overall procedure can also become less automated. This aspect is seen with the general CBR model, particularly with regard to the case-based learning program. A conclusion put forward from the earlier work specific to case-based learning was that continual memory update (i.e., the case memory is reorganised each time a new case is entered) is over-flexible with respect to the dysmorphology domain. For this reason, the CBR assistant employs a more general version of the CBL program that does not continually update the case-base. The CBL program is employed to suggest possible links or generalisations, but specific reorganisations are left to the human expert.

The review of computer applications in dysmorphology concluded three general system categories: databases, ranking systems and expert systems. These three models vary in the scope of their performance tasks, and consequently in their level of automation with respect to diagnosis. Databases allow the construction of a differential diagnosis. They are not designed to suggest or imply a specific diagnosis. Thus, automation of the diagnostic process is minimal. Expert systems are designed to propose a specific diagnosis. A clear aim has been, in these instances, to automate the diagnostic process. This 
may involve some interaction with the user, for example, SDD requests additional information from the user during its cycling procedure, although decisions are made by the program not the human expert. Ranking systems employ a model that lies somewhere between databases and expert systems. In a sense, they suggest a specific diagnosis by displaying its relative score compared to other possibilities according to the chosen formula. With these systems, automation does not so much model the diagnostic procedure, rather it replaces it by a readily available compromise. Like databases, these systems are not designed to make specific decisions, or interact with the user. As well as employing a CBL program to suggest reorganisations to the syndrome nomenclature, the general CBR model presented in this chapter also incorporates a ranking formula which can be used to suggest the most likely hypotheses from a list of candidates. This functionality provides an independent function to the CBR system which can be utilised either as a separate analytical tool, or can be employed within the context of the overall CBR procedure. The ranking function uses the contrast model and is discussed in section 7.3.

The work to date has used the contrast model to assess the similarity between two entities, which has in turn employed the LDDB diagnostic model. Thus, the levels of diagnostic significance encoded within the diagnostic model have a direct effect on the performance of the case-based learning program. Similarly, if the contrast model is also to be used with respect to a ranking function, then the diagnostic model which it employs will also influence its performance. One criticism of the LDDB encoding of diagnostic indicators is that it is merely one interpretation of diagnostic significance. Furthermore, it is a relatively context-free assignment of diagnostic weight. It was highlighted in Chapters 5 and 6 that within the context of a decision between competing hypotheses the physician can assign additional bias to some anomalies whilst ignoring others. The contrast model allows bias to be assigned, but only to feature sets and not to individual dysmorphic features. The CBR assistant model is designed to operate in an iterative and interactive mode, and allow an expert user to account for personal bias with regard to specific anomalies with respect to the current group under study. That is to say, if the user is considering a possible diagnosis (which could involve a ranking procedure), or analysing a potential reorganisation with the CBL program, personal bias can be assigned to individual anomalies via a number of additional utilities that allow the diagnostic model to be altered. This facet of the model is intended to increase the flexibility of the system with regard to user interpretation of diagnostic significance. In a way, this 
type of flexibility is comparable to the generic nature of the case representation. As well as being able to cope with different case representations (from different sources), the CBR assistant model provides the mechanisms to account for different diagnostic interpretations (of dysmorphic features). This is achieved by allowing the expert to alter the significance level of individual abnormalities (i.e., $S_{1} \ldots S_{4}$ ). This does mean, however, that the nomenclature for dysmorphic features remains fixed to the Winter and Baraitser model.

\subsection{A CBR Assistant Model and Procedure}

Figure 7.1 illustrates the CBR assistant design. This model comprises similar functional components to the general CBR model shown by Figure 2.5 of Chapter 2, and the general CBL model illustrated by Figure 5.7 of Chapter 5, along with some additional procedural phases.

The selection of indexes with which to probe the case-base, called the elaborate phase of the CBR procedure, incorporates both human interaction and system guidance. An index comprises a list of features that are evident in the new case. An indexing model contains a small set of simple rules that suggest potentially useful index sets (i.e., a conjunction of dysmorphic feature triplets) for matching against the case-base. This is an additional utility which uses the diagnostic model to create a list of indexes from the features of the new case. The indexing model is discussed in section 7.5. The user interacts directly with the system in this phase by selecting which index to use. The expert also interacts with the elaborate stage in an indirect manner. The indexing model employs the diagnostic model to compile a list of possible indexes, which can in turn be edited by the expert to account for personal interpretation of diagnosticity. A number of utility functions allow the user to alter the suggested indexes or create new indexes. This promotes an iterative process of case-based retrieval. The user may use an index to retrieve a group of matching case or syndrome records from which some may be selected for further investigation, or may be rejected. If the expert is not satisfied with the retrieved case-base entities, the indexes may be edited or new ones created, and the retrieval program re-run. The aim is to arrive at a contextual focus in terms of a group of matching cases and/or syndromes with respect to a set of key abnormalities. That is to say, the iterative procedural phases of elaboration, retrieval, selection and revision of 


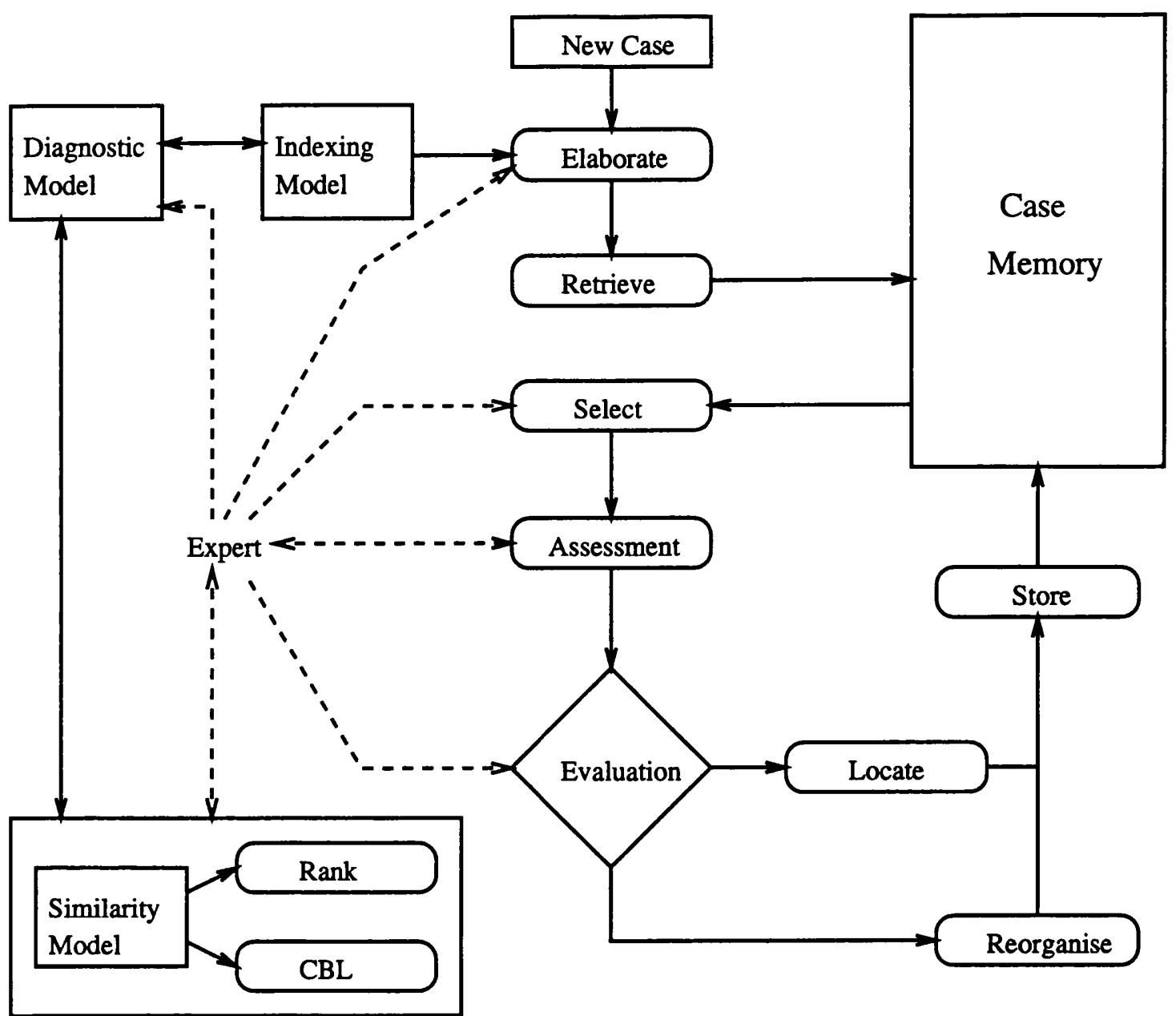

Figure 7.1: A general CBR model for dysmorphology.

indexes are designed to provide an initial list of similar case-base entities, and allow for the personal interpretation (and domain knowledge) of the user.

While constructing this list, the user can examine the retrieved case and syndrome records (through a utility function which lists the recorded features and indicates their significance level according to the diagnostic model). At this point in the CBR procedure the similarity model, incorporating the contrast model, is not yet used. The goal at this phase of the procedure is to arrive at a list of matching entities for further analysis. Thus, the user may decide to reject a case or syndrome from the retrieved list due to a hard abnormality that does not match with the input case. Alternatively, the expert can enter the next phase of operation, called the assessment phase, in which the similarity model can be employed to rank the list of matching entities. The similarity model comprises the CBL program and a ranking formula which both utilise the contrast model. These are 
discussed further in sections 7.4 and 7.3 respectively. The goal of the assessment phase is to allow the CBR assistant to suggest possible solutions through its similarity model. The suggested solution may be the diagnosis that is ranked first in the list. Additionally, the user can employ the CBL program with a localised group within the case memory in order to analyse potential (or suggested) category reorganisations.

Evaluation of possible diagnoses and case memory reorganisation is a human function. A firm diagnosis will indicate a location for the new case in memory, as an instance of a syndrome record (which is managed by linking the instances slot of the syndrome header to the parents slot of the case header). A situation may occur in which no retrieved memory entities match the new case sufficiently in order to link it with either a syndrome or another case. In this instance the new case would simply be stored in isolation. If the best match is with another isolated case, the expert can create a new generalised memory record (with the object-type slot set to NODE) beneath which the two matching cases are linked. It is the aim of the CBL program to indicate these types of links and suggest the format of the new general node. Even if the new case is linked below an existing syndrome, the user is still allowed to edit the syndrome description if it is deemed appropriate. The additional functionality provided for user construction and reorganisation of case memory is described in section 7.4.3.

The general mode of operation with the CBR assistant model is, thus, interactive. It is a form of case-based decision aiding in which intermediate and final decisions are made by the expert, but in which index elaboration, indexing and retrieval, similarity assessment (incorporating a case-based learning operation) and evaluation are all automated to some degree by the CBR assistant.

\subsection{Ranking Hypotheses with the Contrast Model}

Chapter 3 described a distributed architecture for a case-base, and gave an example of how an indexing technique could be employed to retrieve a differential diagnosis against a case-base of 1885 syndromes. This indexing procedure constitutes part of the iterative phase of elaboration, retrieval and selection illustrated by Figure 7.1. Whilst the expert can examine retrieved cases or syndromes in order to refine the selection, an additional function has been developed to enable a ranking operation similar in nature to that performed by SYNDROC and the system developed by Weiner et. al., and which can also 
be compared to the GENDIAG approach. With these systems, a differential diagnosis is effectively constructed by listing those syndromes that satisfy a numerical condition on application of the ranking formula. In a sense, the highest scoring syndrome is the suggested diagnosis. With the CBR assistant, the ranking procedure can be used in two ways, either to compare the selected syndrome and case records that have been returned through an initial indexing phase, or to actually construct the differential diagnosis by application of the ranking formula across all syndromes in the case-base (i.e., an equivalent mode to SYNDROC). Both Schorderet and Weiner applied a numeric formula exhaustively against the syndrome database in order to arrive at the most probable candidates. With the CBR assistant model, the user specifies the case-base records with which to compare the new case. This list could comprise those cases and syndromes selected via the first phase of CBR operation or, as with the Schorderet and Weiner approach, could comprise all syndrome records in the case-base.

The ranking formula utilised in this instance is a version of the contrast model. Chapter 6 has already discussed the applicability of Tversky's contrast model versus the inapplicability of probability formulae such as Bayes' rule when applied to the dysmorphology domain, and these arguments apply also with respect to the selection of the contrast model as a ranking formula. Unlike SYNDROC, the contrast model does not rely on statistical assumptions or scores that are calculated from the existing database population (creating a pseudo-Bayesian formula). Not unlike Weiner's system, which calculates scores for and against each hypothesis, Tversky's model calculates each score as a contrast between matching and distinctive features. Furthermore, as with GENDIAG, the extended contrast model utilises different levels of diagnostic significance to account for feature salience. The applicability of the contrast model as a ranking formula is further enhanced by the fact that it can be set for asymmetrical matching, i.e., it can have a diagnostic bias in its assessment of similarity. That is to say, ranking is a diagnostic procedure which relates to matching with an asymmetrical direction. Thus, the variation of the contrast model used within the ranking formula is one in which similarity takes precedence over dissimilarity, which is essentially the evaluate function described in Chapter 6. The ranking formula actually incorporates the extended contrast model shown by Equation 6.2 with parameter settings such that $\theta>\alpha>\beta$.

To demonstrate the operation of the ranking function, the following section describes an experiment similar to those performed by Schorderet $[66,68]$, in which the whole 
database of syndrome records is exhaustively compared to an input case in order to arrive at a differential diagnosis.

\subsubsection{Experiments with the Ranking Model}

The twenty-eight cases comprising the Acrocephalosyndactyly data set shown in Table 4.2 (including the cases of Nager, Treacher-Collins Syndrome and Acrofacial Dysostosis) were each matched against a case-base of the 1885 LDDB syndromes (i.e., those casebase entities denoted by SYNDROME in the object-type slot of the header). A ranking function consisting of the formula shown by Equation 6.2 was utilised. Initially, the parameters of the formula were set to the values shown in Figure 7.2. A differential diagnosis comprised those candidates that achieved a contrast-score greater or equal to the evaluation threshold, $E_{t}$. The parameter settings shown in Figure 7.2 resulted in the differential diagnoses described by Table 7.1, which shows the size of the differential list returned by the ranking function, and an indication of the position of the correct diagnosis (if included in the differential list).

$$
\begin{array}{r}
\theta=1.0, \quad \alpha=0.4, \quad \beta=0.2, \quad E_{t}=24.0 \\
f_{i} \rightarrow W_{i}= \begin{cases}12 & \text { if } i \in S_{4} \\
5 & \text { if } i \in S_{3} \\
1 & \text { otherwise }\end{cases} \\
f_{j} \rightarrow W_{j}= \begin{cases}12 & \text { if } j \in S_{4} \\
5 & \text { if } j \in S_{3} \\
0 & \text { otherwise }\end{cases} \\
f_{k} \rightarrow W_{k}= \begin{cases}12 & \text { if } k \in S_{4} \\
5 & \text { if } k \in S_{3} \\
0 & \text { otherwise }\end{cases}
\end{array}
$$

Figure 7.2: Ranking contrast parameters.

Referring to Table 7.1, the ranking procedure has performed well with the fifteen cases of Acrocephalosyndactyly (i.e., Apert, Pfeiffer and Saethre-Chotzen syndromes). 


\begin{tabular}{|l|l|l|l|}
\hline Case & Diagnosis & Differential Size & Diagnosis Rank \\
\hline 6761 & Apert & 13 & 1st \\
616929 & Apert & 11 & 1st \\
618717 & Apert & 7 & 1st \\
606067 & Apert & 8 & 1st \\
564525 & Apert & 12 & 1st \\
5462 & Saethre-Chotzen & 10 & 1st \\
7464 & Saethre-Chotzen & 1 & 1st \\
$6150 \mathrm{a}$ & Saethre-Chotzen & 5 & $1 \mathrm{st}$ \\
7385 & Saethre-Chotzen & 2 & st \\
7877 & Saethre-Chotzen & 4 & 1st \\
0001 & Pfeiffer & 19 & 2nd \\
$5286 \mathrm{a}$ & Pfeiffer & 4 & 1st \\
$5286 \mathrm{~b}$ & Pfeiffer & 3 & 1st \\
$5286 \mathrm{c}$ & Pfeiffer & 1 & 1st \\
4200 & Pfeiffer & 10 & 3rd \\
8175 & Nager & Nil & Not Listed \\
6566 & Nager & 7 & Not Listed \\
7031 & Nager & 4 & 4th \\
0005 & Nager & 2 & Not Listed \\
5754 & Nager & 21 & 1st \\
0002 & Acrofacial Dysostosis & 22 & 1st \\
0003 & Acrofacial Dysostosis & 21 & 1st \\
0004 & Acrofacial Dysostosis & 22 & 1st \\
9605 & Acrofacial Dysostosis & 23 & 2nd \\
6351 & Treacher-Collins & Nil & Not Listed \\
6638 & Treacher-Collins & Nil & Not Listed \\
8967 & Treacher-Collins & Nil & Not Listed \\
7312 & Treacher-Collins & Nil & Not Listed \\
\hline
\end{tabular}

Table 7.1: Position of correct diagnosis in ranked differential for Acrocephalosyndactyly and Acrofacial Dysostosis cases.

The correct diagnosis has been listed first for thirteen of these cases, and within the top three for the other two cases. The size of the differential list varies from only one (Cases 7464 and 5286c), for which the diagnosis is correct, to nineteen (Case 0001) for which the correct diagnosis is listed second. The ranking function has also performed particularly well with the four cases of Acrofacial Dysostosis with Post-Axial Defects, for which, with an average differential list of twenty-two syndromes, the correct diagnosis is ranked either first or second. The other members of this group have not faired so well, however (i.e., cases of Nager and Treacher-Collins syndrome). Of the four cases of Treacher-Collins syndrome, no case-base syndromes have achieved the evaluation threshold, and thus, the differential list is empty. A similar situation has occurred for one case of Nager Acrofacial Dysostosis (Case 8175). The other four cases of Nager syndrome do have differential diagnoses, but two of these (Cases 6566 and 0005) do not list the correct diagnosis 


\begin{tabular}{|l|l|l|l|}
\hline Case & Diagnosis & Differential Size & Diagnosis Rank \\
\hline 6761 & Apert & 21 & 1st \\
616929 & Apert & 20 & 1st \\
618717 & Apert & 17 & 1st \\
606067 & Apert & 16 & 1st \\
564525 & Apert & 20 & 1st \\
5462 & Saethre-Chotzen & 16 & 1 st \\
7464 & Saethre-Chotzen & 1 & 1 st \\
$6150 \mathrm{a}$ & Saethre-Chotzen & 10 & 1st \\
7385 & Saethre-Chotzen & 4 & 1st \\
7877 & Saethre-Chotzen & 5 & 1st \\
0001 & Pfeiffer & 31 & 2nd \\
$5286 \mathrm{a}$ & Pfeiffer & 10 & 1st \\
$5286 \mathrm{~b}$ & Pfeiffer & 6 & 1st \\
$5286 \mathrm{c}$ & Pfeiffer & 6 & 1st \\
4200 & Pfeiffer & 24 & 3rd \\
8175 & Nager & 3 & 2nd \\
6566 & Nager & 7 & Not Listed \\
7031 & Nager & 6 & 4th \\
0005 & Nager & 4 & 4th \\
5754 & Nager & 25 & 1st \\
0002 & Acrofacial Dysostosis & 22 & 1st \\
0003 & Acrofacial Dysostosis & 22 & 1st \\
0004 & Acrofacial Dysostosis & 22 & 1st \\
9605 & Acrofacial Dysostosis & 25 & 2nd \\
6351 & Treacher-Collins & Nil & Not Listed \\
6638 & Treacher-Collins & 1 & Not Listed \\
8967 & Treacher-Collins & Nil & Not Listed \\
7312 & Treacher-Collins & Nil & Not Listed \\
\hline
\end{tabular}

Table 7.2: Position of correct diagnosis in ranked differential for Acrocephalosyndactyly and Acrofacial Dysostosis cases with $E_{t}=20.0$.

(although it is worth noting that these differentials did include related diagnoses, i.e., syndromes related to Nager, for instance, Acrofacial Dysostosis was listed first for Case 0005). Two cases of Nager syndrome, Case 7031 and Case 5754, have the correct diagnosis ranked at fourth and first respectively.

A second test of the ranking procedure was performed with the evaluation threshold lowered to 20.0. The results from this run-through are shown in Table 7.2. In this instance, the ranking function returns a differential diagnosis for those cases of Nager syndrome for which it had previously failed (although for Case 6566 the correct diagnosis is still not listed, but Acrofacial Dysostosis is ranked second). The trade-off in lowering the evaluation threshold is that the differential diagnoses become larger overall (although the rankings do not change). As with the process of adjusting a boolean index to return a manageable differential, the adjustment of desirable system parameters is an iterative, 
empirical process. In this sense, the selection of a suitable threshold level performs a similar function to the selection of a desired tightness or looseness of an index. However, the extended contrast model has an additional advantage in that it accounts for the salience of matching and unmatching abnormalities. The additional weights that can be allocated to the four defined significance levels depending on the matching set (i.e., $A \cap B, A-B$ or $B-A$ ), allow additional bias to be attributed accordingly.

\subsubsection{Discussion}

The development of a ranking function has not provided a major focus for this thesis. However, the experiments described in section 7.3.1 are intended to demonstrate two points. Firstly, if a comprehensive database of syndromes (defined by a representation common to presented cases) is available, then the application of a ranking formula is relatively straightforward. The scope of a ranking function is really only to assist in creating a differential diagnosis, although if the user is inexperienced such a tool may prove useful by focusing the physicians attention on specific candidates within the differential list. Secondly, the experiments are designed to show the applicability of the contrast model as a ranking formula in dysmorphology. Whereas Bayes' formula and its derivatives rely on comprehensive statistical data, which is not generally available in dysmorphology, the contrast model relies on a comprehensive assessment of diagnostic salience which, in this instance, is readily available.

It is difficult to quantitatively define success and failure with regard to creating a differential diagnosis, whether ranked or not. Certainly, success should correspond at least to a differential that lists the correct diagnosis, but how large or small should a differential diagnosis be? And, if the differential diagnosis is ranked, then what position in the ranking defines success? Previous systems appear to have adopted fairly arbitrary approaches with regard to these questions. Databases such as LDDB, DYSMOR and BDIS rely on the user to retrieve a 'sensibly' sized differential as defined by the user. El Busca defines its differential diagnosis by the top twelve ranked syndromes. SYNDROC defines its differential limit as half the value of the highest achieved certainty coefficient, whereas Weiner's system includes those syndromes with a probability score of 0.85 or more. GENDIAG initially creates a differential diagnosis from those syndromes that match the case on a grade 3 anomaly (this is equivalent to indexing LDDB with one hard abnormality). The differential is then ranked by the corresponding belief function 
of each syndrome.

In the examples of section 7.3.1, those syndromes that achieve a contrast-score that reaches the evaluation threshold constitute the differential diagnosis. No attempt is made here to quantify success or failure. Provided the size of the differential is sensible (i.e., small enough such that an experienced physician is sufficiently focussed on candidate diagnoses in order to proceed with further investigations) then the ranking is not considered all that important, particularly because of the limitations of the diagnostic model and the fact that the ranked differential diagnosis is only based on symbolic data. With larger differentials (say 10 or more syndromes) then an indication of the relative similarity score could be useful to refine this list further, especially if the user is a less experienced physician.

The review of computer applications in dysmorphology in Chapter 2 noted that with previous ranking systems there has been no attempt to compare the results of the respective ranking formula with a standard boolean indexing mechanism. This type of comparison can demonstrate an important problem when a ranking approach is used solely to form the differential diagnosis, i.e., the correct syndrome may not be listed. There will be a greater chance of this occurrence if the ranking formula accounts for both similarity and dissimilarity, as it does with the contrast model and Weiner's use of a likelihood score based on probability values for and against the hypothesis [82, 81]. Whilst these formulae present a more balanced model of comparison, the non-uniform nature of case descriptions might cause the correct syndrome to be omited from the differential diagnosis due to the weight of distinctive features. The extended contrast model described in Chapter 6 is designed to allow additional weight to be attributed to the relevant key anomalies, and thus to account for this type of problem. However, to reiterate what has already been discussed in section 7.2 , it may be necessary for the expert to vary the weights according to the context of the current group under study whilst the first iterative phase of the CBR procedure takes place, i.e., the creation of the differential diagnosis.

The second experiment of section 7.3.1 demonstrates one type of empirical adjustment. The evaluation threshold was lowered because the ranking formula failed to generate a differential diagnosis which contained the correct syndrome for seven of the twenty-eight cases, specifically cases of Nager Acrofacial Dysostosis and Treacher-Collins syndrome. This adjustment proved successful for two cases of Nager: Case 8175 and 


\begin{tabular}{|l|l|l|l|}
\hline Case & Diagnosis & Differential Size & Diagnosis Rank \\
\hline 8175 & Nager & 12 & 2nd \\
6566 & Nager & 11 & Not Listed \\
7031 & Nager & 26 & 3rd \\
0005 & Nager & 7 & 4th \\
5754 & Nager & 32 & 1st \\
6351 & Treacher-Collins & 10 & 3rd \\
6638 & Treacher-Collins & 9 & 2nd \\
8967 & Treacher-Collins & Nil & Not Listed \\
7312 & Treacher-Collins & 8 & 2nd \\
\hline
\end{tabular}

Table 7.3: Position of correct diagnosis in ranked differential for Nager and TreacherCollins cases with $\theta=1.5$.

Case 0005, but still failed for the other 5 cases which included all the cases of TreacherCollins syndrome and one case of Nager Acrofacial Dysostosis. An alternative adjustment could be to increase the relative contribution of common abnormalities (those features in the set $A \cap B$ ) by increasing the value of $\theta$. By setting $\theta$ equal to 1.5 (and with the evaluation threshold at 24.0) a differential diagnosis containing the correct syndrome is generated for three more of the Treacher-Collins cases. Table 7.3 indicates how this adjustment has revised the effect of the ranking formula on cases of Nager Acrofacial Dysostosis and Treacher-Collins syndrome.

The ranking formula still fails to produce a differential diagnosis for Case 8967, which is known to be an instance of Treacher-Collins syndrome, and Case 6566, which is a case of Nager Acrofacial Dysostosis. Case 8967 comprises the dysmorphic features shown in Table 7.4, which indicates the diagnostic significance of each anomaly according to the diagnostic model. The only feature listed within this case description that belongs to the set of highest diagnosticity $\left(S_{4}\right)$ is (ears external-ears cystic-ear-pinna), but this anomaly is not specifically listed within the record for Treacher-Collins syndrome. External ear anomalies are listed in the Treacher-Collins syndrome description, so this could possibly demonstrate an error of interpretation (however, as the cystic ear pinna anomaly carries the highest level of significance one would expect careful examination of this feature before recording it within the case description). The practical implication of this is that the description of Case 8967 is misleading for a numeric formula which accounts for both common and distinctive features, and which compares a similarity score to a threshold level in order to form the differential diagnosis. An indexing approach, however, might suffer less from the variability of case descriptions. In keeping with the 


\begin{tabular}{|l|l|}
\hline Feature & Diagnostic Set \\
\hline (ears external-ears cystic-ear-pinna) & $S_{4}$ \\
(ears external-ears low-set-ears) & $S_{2}$ \\
(eye-structures palpebral-fissures & \\
palpebral-fissures-slant-down) & $S_{3}$ \\
(nose general convex/beaked-profile-of-nose) & $S_{3}$ \\
(face mandible small-mandible/micrognathia) & $S_{2}$ \\
(face maxilla hypoplastic-maxilla-excl-malar-reg) & $S_{3}$ \\
(neurology general normal-intelligence) & $S_{1}$ \\
\hline
\end{tabular}

Table 7.4: Case 8967 (Treacher-Collins syndrome).

\begin{tabular}{|l|l|}
\hline Syndrome & Contrast-Score \\
\hline SAY [1986] & 9.0 \\
ANYANE-YEBOA [1987] & 9.0 \\
THONG [1978] & 4.5 \\
TREACHER-COLLINS & 3.0 \\
PIEPKORN [1977] & 3.0 \\
MAUMENEE [1977] & 3.0 \\
KAPLAN [1988] & 3.0 \\
FUJIMOTO [1987] & 1.5 \\
ARMENDARES [1974] & 1.5 \\
\hline
\end{tabular}

Table 7.5: Ranked differential diagnosis for Case 8967.

Winter and Baraitser guidelines for indexes, the index:

$$
\begin{aligned}
& \text { ((face mandible small-mandible/micrognathia) } \\
& \text { (nose general abnormal) (ears external-ears abnormal)) }
\end{aligned}
$$

which is derived from the description of Case 8967, can be used to return a list of nine syndromes (by using the Retrieve function described in Chapter 3) which includes Treacher-Collins syndrome. Table 7.5 shows the retrieved syndromes ranked along their respective contrast-scores (with $\theta=1.5$ ), all of which fall some way below the evaluation threshold $\left(E_{t}=24.0\right)$.

The main practical difference between indexing and ranking is that a boolean index will return a matching syndrome regardless of the significance of individual features. Thus, if with a ranking procedure the correct syndrome fails to reach the respective criterion for inclusion in the differential diagnosis, with an indexing mechanism the correct syndrome may still be returned by the program. The conclusion to be drawn from this is that it may be better to use indexing first in order to create an initial list of candidates, rather than purely using a ranking function. This is the approach adopted with the 
GENDIAG system [23]. Either way, the process of selecting a satisfactory differential diagnosis (or one in which the physician is satisfied that correct syndrome is listed) might necessarily be an iterative one which incorporates empirical judgement and adjustment of system parameters.

\subsection{Case-Based Learning with the Case-Based Assistant Model}

The experiments reported in chapters 4,5 and 6 demonstrated how a case-based learning algorithm may be employed to both generate and operate a case database, or case-base, and chapter 3 described the underlying linkage which enables a localised hierarchy to be developed within the distributed architecture. In each of the case-based learning experiments, the case-base evolved through incremental input of individual cases, and memory organisation was constructed dynamically, or automatically, by the CBL program. The starting point for these experiments was an empty, or null database. This type of operation is characteristic of an incremental concept formation approach in which a set of cases is initially used to create a concept hierarchy, and the remaining test cases are used to evaluate program performance. The algorithmic functions of incremental concept formation and case-based learning are very similar. However, the emphasis of the performance task of the two approaches is different. Incremental concept formation is primarily concerned with the evaluation of generated concepts and category structure is critical to such programs. Case-based reasoning is primarily concerned with using its case database to solve a related problem (through an associated assessment of similarity). CBR systems that automate learning do rely more on case memory structure than CBR systems that don't learn. A conclusion to be drawn from this is that for a general CBR system the case-base would not necessarily be developed from an empty database, but category organisation is desirable if the system is to automate learning. This type of model is effectively what has been developed in this instance. The case-based assistant model comprises a memory architecture which allows localised hierarchies to be constructed whilst providing an efficient mechanism for standard case-based retrieval (with confined search).

Much of general CBR research has not required the creation of a case memory from a null starting point. In fact, more often than not, the case-base will be pre-compiled. 
Unless the specific goals of the project involve learning and automatic reorganisation of case memory from a null database (as is the case with incremental concept formation systems), a pre-compiled case-base will serve as a platform for the development of the system, which in turn may focus on specific research topics relating to CBR. The research focus will will depend to a great extent on the domain and style of CBR, for example, in a problem solving domain there may be an emphasis on adaptation of past cases. The research focus of this thesis is learning, but the background goal of developing a casebased system that is a practical and useful tool for a domain expert has resulted in the creation of the more general CBR model described in this chapter. This development is not retrospective. The higher order goal of practicality was the main influence on the design of the case-based architecture described in Chapter 3. A consequence of these factors is that the case-based assistant model incorporates two modes of case-based learning: a stand-alone CBL program such as C-UNIMEM, and a generalised version of CBL which operates within the context of an interactive procedure. With this model, a case memory comprising syndromes and cases will already exist. The existing case-base represents the current state of the dysmorphology domain. It has not been generated by a case-based learning program, rather it is a pre-compiled database of what is currently understood with regard to syndrome nomenclature. This does not mean that a standalone case-based learning program as described by Chapters 4 through 6 does not provide a practical tool. The ability to run a CBL program such as C-UNIMEM against a set of cases is still regarded as a valid option with regard to the case-based assistant model. However, such an experiment would take place in isolation from the main case-memory. The pre-compiled case-base comprises four record types which are organised and stored according to the distributed architecture described by Chapter 3.

- Syndromes, which are denoted by the SYNDROME string in the object-type slot of the header object.

- Diagnosed Cases, which are denoted by the CASE string in the object-type slot of the header object, and which have a link from the parents slot of their header object to the instances slot of the respective syndrome record.

- Undiagnosed Cases, which are denoted by the CASE string in the object-type slot of the header object, but do not have any links to syndrome records, and thus stand isolated within the case-base. 
- Nodes, which are denoted by the NODE string in the object-type slot of the header object, and which can refer to two types of entity. A node can represent the generalised description between two linked cases, in which case the necessary links will exist between the instances slot of the node and the parents slots of the relevant cases. A node can also represent a generalised syndrome family record, which will have links from its children slot to the parents slots of relevant syndrome header objects.

The overall structure of the case-base is thus distributed, but localised hierarchies are derived through links between header objects (see Figure 3.9 of Chapter 3 ). The localised hierarchies comprise three possible levels: a top-level node which represents a syndrome family, a second level comprising of sibling syndromes and cases that are linked to the syndrome group (but have no firm diagnosis), and a third level with diagnosed cases. Unlike the four levels of the dysmorphology syndrome nomenclature illustrated by Figure 3.1 of Chapter 3, there is no root node. Consequently, undiagnoses cases stand isolated. Cases can be linked to syndrome family nodes in order to represent undiagnosed cases that are thought to belong to the respective syndrome group, but significant dissimilarity between the case and the sibling syndromes prevents a firm diagnosis (promoting learning within the in context, or with reference to, localised syndrome families).

\subsubsection{Interactive CBL Procedure}

The case-base organisation described above does not incorporate a root node at which to commence search downwards with the CBL program. This is an important point with respect to the CBR assistant model. The case memory does not consist of one category hierarchy with one top-level starting point for search. The CBL procedure is initiated with respect to localised hierarchies. The first phase of CBR operation is designed either to derive a differential diagnosis, and subsequently a diagnosis through human decision making, or, if no diagnosis is forthcoming, to focus the user's attention on similar cases in memory. Human analysis of the selected list of case-base entities (from the first phase of CBR operation) is required to identify starting points for CBL operation. For instance, the first phase of CBR operation may result in a number of retrieved (and possibly ranked) entities such as syndromes (against which a firm diagnosis could not be made), diagnosed cases (which would indicate a possible syndrome) and undiagnosed cases. If a number of matching records pertain to a related group, i.e., a specific syndrome plus 


\begin{tabular}{|l|l|}
\hline Feature & Diagnostic Set \\
\hline (cranium cranial-sutures craniosynostosis) & $S_{4}$ \\
(cranium general brachycephaly) & $S_{3}$ \\
(cranium general acro/turricephaly) & $S_{3}$ \\
(ears external-ears posteriorly-rotated-ears) & $S_{2}$ \\
(eyes general prominent-eyes-including-proptosis) & $S_{3}$ \\
(eyes general hypertelorism) & $S_{3}$ \\
(eye-structures palpebral-fissures & \\
palpebral-fissures-slant-down) & $S_{3}$ \\
(nose general small/short-nose) & $S_{2}$ \\
(face general flat-face) & $S_{2}$ \\
(face maxilla hypoplastic-maxilla-excl-malar-reg) & $S_{3}$ \\
(hands fingers osseous-syndactyly-of-fingers) & $S_{3}$ \\
(neurology general normal-intelligence) & $S_{1}$ \\
\hline
\end{tabular}

Table 7.6: Case 564525 dysmorphic features.

one or more cases diagnosed as that syndrome, then a localised CBL procedure could be initiated with the syndrome record providing the effective top-level entry point (or root node).

The following example demonstrates this more general use of a case-based learning program within the context of the CBR assistant model. It utilises a pre-compiled casebase incorporating the 1885 LDDB syndrome prototypes and twenty-seven of the Acrocephalosyndactyly case data set with links constructed between the header objects of the 'diagnosed' cases and the respective syndrome records. For the purposes of this example, one case from the Acrocephalosyndactyly data set has not been included in the existing case-base, Case 564525, which has a known diagnosis of Apert (Acrocephalosyndactyly Type I). Case 564525 comprises the dysmorphic features shown in Table 7.6.

The indexing model creates a list of possible indexes from the description of Case 564525 based on its simple rule set (which is discussed in Section 7.5.1). One of the listed indexes comprises the feature conjunction:

\section{((cranium cranial-sutures craniosynostosis) \\ (hands fingers abnormal) (eyes general abnormal))}

which, when used with the Retrieve program, returns the case-base objects listed in Table 7.7.

Following the retrieval and selection phases of the CBR procedure, the user may choose to apply the ranking function against selected syndrome records. If the ranking function is applied with respect to Case $\mathbf{5 6 4 5 2 5}$ and the twenty-four syndromes listed in 


\begin{tabular}{|l|l|l|}
\hline TYPE & MEMORY ID & NAME \\
\hline CASE & 218 & 606067 (Apert) \\
CASE & 206 & 6150a (Saethre-Chotzen) \\
CASE & 200 & 618717 (Apert) \\
CASE & 197 & 616929 (Apert) \\
CASE & 196 & 5462 (Saethre-Chotzen) \\
CASE & 193 & 6761 (Apert) \\
SYNDROME & 188 & WOON [1980] \\
SYNDROME & 181 & WEAVER [1987] \\
SYNDROME & 156 & SHPRINTZEN [1982] \\
SYNDROME & 146 & SAKATI-NYHAN \\
SYNDROME & 145 & SAETHRE-CHOTZEN \\
SYNDROME & 129 & PFEIFFER \\
SYNDROME & 106 & LOPEZ-HERNANDEZ \\
SYNDROME & 97 & KAPLAN [1988] \\
SYNDROME & 92 & JACKSON-WEISS \\
SYNDROME & 89 & IDAHO II \\
SYNDROME & 79 & HERRMANN-PALLISTER \\
SYNDROME & 75 & HARPER [1967] \\
SYNDROME & 63 & FURLONG [1987] \\
SYNDROME & 35 & CRANIOFRONTONASAL \\
SYNDROME & 33 & CRANIOECTODERMAL DYSP \\
SYNDROME & 30 & COH \\
SYNDROME & 29 & CLEFTING-COLOBOMA-MR \\
SYNDROME & 28 & CHRISTIAN [1971] \\
SYNDROME & 26 & CARPENTER \\
SYNDROME & 14 & BALLER-GEROLD \\
SYNDROME & 11 & ARMENDARES [1974] \\
SYNDROME & 10 & APERT \\
SYNDROMEE & 9 & ANYANE-YEBOA [1987] \\
SYNDROME & 3 & ACS-WAARDENBURG \\
\hline
\end{tabular}

Table 7.7: Memory items which list craniosynostosis, abnormal fingers, and general eye abnormalities.

Table 7.7, and with the relevant parameters set to those levels shown in Figure 7.2, then Apert syndrome is ranked first with a contrast-score of 45.0 (Jackson-Weiss syndrome is ranked equal second with Saethre-Chotzen syndrome, both with a contrast-score of 32.0). Thus, further computer assisted analysis using case-based learning may not be necessary in this instance. However, in order to demonstrate the CBL procedure, let us assume that the user does desire further analysis. An important user interaction at this stage is an analysis of the retrieved objects listed in Table 7.7, which incorporates both diagnosed cases and syndromes. The user's attention should be drawn to the fact that all the listed diagnosed cases are of Apert or Saethre-Chotzen type Acrocephalosyndactyly, and both these syndromes are also listed. The conclusion to be drawn from this is that for the purposes of CBL, the Apert and Saethre-Chotzen records provide the possible 
top-level entry points to localised hierarchies.

Figure 7.3 illustrates the application of the CBL program against these two localised hierarchies. The Apert and Saethre-Chotzen records effectively provide a child list of an imaginary root node. Each syndrome has in turn a list of stored instances, i.e., diagnosed cases. Figure 7.3A shows the localised hierarchical structures that are derived through the relevant links between individual case and syndrome header objects. The CBL program evaluates with each syndrome record (with the evaluation threshold and system parameters equal to those listed in Figure 6.2 of Chapter 6, i.e., those utilised with C-UNIMEM). Each syndrome record has no associated children, but does have a list of instances (or diagnosed cases) against which to apply the generalise function. Appendix D lists a trace of the CBR assistant procedure with regard to Case 564525. When the generalise function is invoked with Case 564525 and the respective instances of the Apert syndrome record, each match is positive, resulting in four potentially new children. The dysmorphic features that constitute a new child are listed (i.e., the common features of Case 564525 and the respective instance). The interactive version of the CBL program does not automatically form the new nodes, rather it informs the user of the possible reorganisation. When the generalise function is applied to the instances of Saethre-Chotzen Acrocephalosyndactyly, two new children are proposed with respect to Cases 5462 and 7385. Figure 7.3B illustrates the reorganisations proposed by the CBL program and the common features of each positive generalisation are listed by the program trace in Appendix D.

\subsubsection{Discussion}

The example above demonstrates an effect of the over-flexible nature of stand-alone CBL programs such as UNIMEM as discussed in Chapter 6. That is to say, when the input case matches sufficiently with more than one instance of a node (or diagnosed case of a syndrome), it generalises a new node with each respective instance. With Case 564525, the suggested reorganisation is to generalise six new nodes, and in the case of Apert syndrome, to create new nodes with each diagnosed case. A stand-alone program such as UNIMEM would perform these actions automatically. Interactive processing allows a common-sense decision to be made by the expert. Clearly, Case 564525 should be either diagnosed as Apert type Acrocephalosyndactyly and linked to the Apert syndrome record accordingly (and with a possible generalisation to the Apert syndrome description), or, if 


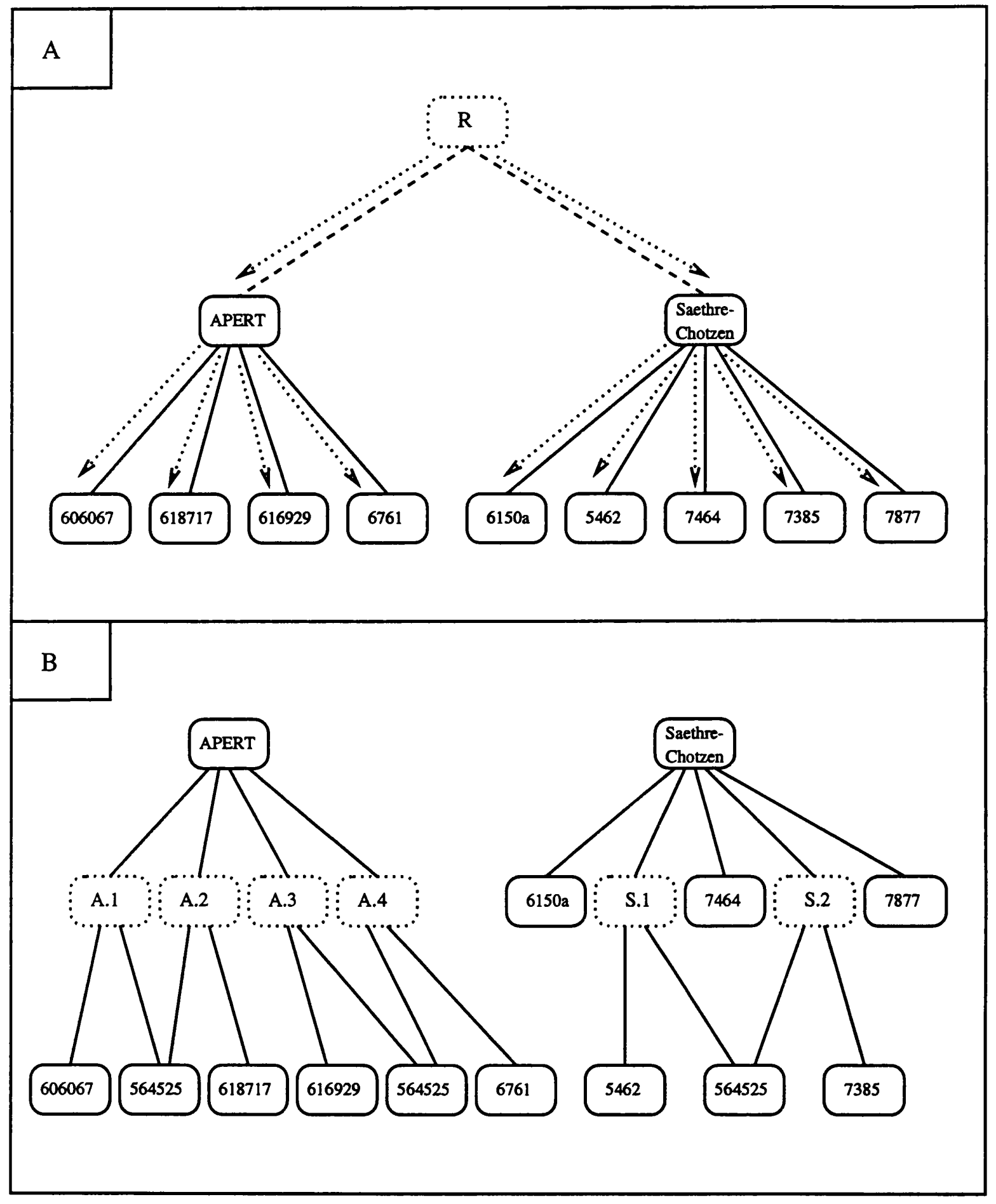

Figure 7.3: Application of CBL to localised sections of the case-base. 
on examination of the relevant matching abnormalities the expert decides to reorganise the Saethre-Chotzen group, then perhaps the creation of one new node abstracted from the entire group would provide a more practical option. The decision to reorganise category information is an important decision that can effect later processing. Furthermore, in the real world such dynamism with respect to the organisation of a syndrome and case database is relatively infrequent. Thus, whilst a CBL procedure can assist the expert in such analysis, the interactive version of the program does not perform continual memory update.

\subsubsection{Additional Functionality}

For the purposes of interactive ranking and learning with the CBR assistant model, a number of additional user functions have been developed.

- Menu System. This work has not been involved with human-computer interfaces, and has accordingly developed a simple menu interface for the CBR assistant model using Common Lisp. The interface lists the respective options for each menu, some of which link to sub-menus. The user is prompted to enter a single keystroke in order to select an option (see Appendix D).

- Memory Edit Functions. A number of editing functions are provided to alter the structure of the case-base. The user can add or delete features to a case or syndrome description (which will in turn add or delete a CLOS object if necessary), and create links between the parents, children and instances slots in header objects. New node objects can be created from a given feature set, and syndromes, cases and nodes may also be deleted. An additional function allows the user to make a specific feature general, i.e., set the lowest sub-category of the feature triplet to abnormal. The 'current' case-base structure can be saved and reloaded.

- Parameter Edit Functions. The ability to alter the relevant system parameters for the purpose of iterative analysis through the ranking and CBL procedures is provided by a number of editing functions.

- Viewing Functions. Throughout system operation, the user is able to view in detail (i.e., list specific features and header details) any selected case, syndrome or node. There are also functions that list the common and distinctive abnormalities 
between a set of two or more records. This can be of particular importance if the selected entities for analysis are undiagnosed (and hence isolated) cases. In this instance, there is no natural starting point for CBL operation and analysis of the common and distinctive features between the input case and the retrieved cases provide the means for human assessment of similarity.

\subsection{Additional Utilities}

\subsubsection{The Indexing Model}

The design of the stand-alone CBL programs described in Chapters 5 and 6 incorporates a body of domain knowledge in the form of the diagnostic model of LDDB. In this sense, these programs (W-UNIMEM and C-UNIMEM) are a form of intelligent knowledge-based system. The case-based assistant model is also a knowledge-based system, and knowledge is stored both within the case (and syndrome) representation, and within the diagnostic model. Furthermore, the CBR assistant design incorporates additional input of domain knowledge from the user through expert decisions. The indexing model is another link in the CBR system that is intended to allow the use of domain knowledge. Its design with respect to this work is very simple, and is meant only to highlight a point in the CBR model's procedure at which domain knowledge may be further incorporated into the system.

The function of the indexing model is to automate the selection of useful indexes for use with the retrieval phase of the CBR procedure. The current design follows the Winter and Baraitser suggestion of forming an index with one hard abnormality with two general features. A function separates the case description according to the four levels of the diagnostic model. Each hard anomaly (or feature with a diagnostic significance of $S_{4}$ ) is grouped with two 'generalised' features which are of different anatomical regions and which belong to the diagnostic set $S_{3}$, i.e., these features are transformed into the format (item attribute abnormal). The resulting combinations of dysmorphic features that satisfy these conditions are listed for analysis and selection by the user. Appendix D shows the indexes elaborated by the program for Case $\mathbf{5 6 4 5 2 5}$. The description of Case 564525 contains one hard anomaly, (cranium cranial-sutures craniosynostosis). The remaining $S_{3}$ features are generalised and grouped to form pairs by region and attribute, i.e., the features (hands fingers abnormal), (cranium 
general abnormal), (eyes general abnormal), and (face maxilla abnormal) produce six possible combinations, each of which forms an index with the conjunction of (cranium cranial-sutures craniosynostosis). This list is presented to the user for analysis and selection.

This simplistic version of an indexing model is not intended especially to propose a good application of domain knowledge, rather it is meant to demonstrate a specific point in the system at which another domain knowledge model may be beneficial. With a comprehensive feature nomenclature in place, an indexing model could provide a useful tool, especially to an inexperienced physician. The model described effectively uses the diagnostic model with one simple rule. A more in depth indexing model may comprise additional knowledge concerning dysmorphic features such as feature dependencies, or commonly co-occurring features. The point is that the selection of good indexes is a very important phase of diagnostic procedure when using a computer system, and may provide the optimum juncture in the program procedure at which to concentrate effort in compiling and applying domain knowledge.

\subsubsection{Editing the Diagnostic Model}

As mentioned above, the major source of domain knowledge in this work has been the comprehensive dysmorphic feature nomenclature and associated diagnostic model. Accordingly, the three main functions with respect to the CBR assistant model utilise this knowledge: the case-based learning program, the ranking program and the indexing model. Thus, the ability to edit the diagnostic model is a crucial aspect of the interactive design. By altering the diagnostic level of individual dysmorphic features, the expert can apply personal bias to the group under study, and subsequently influence the performance of CBL and ranking functions. To enable such user guidance, a number of editing functions have been developed that allow the user to promote or demote any specific feature according to the four significance levels $S_{1} \ldots S_{4}$.

\subsection{Conclusions}

Case-based reasoning has aspects to its theoretical model that are similar to other disciplines in artificial intelligence, but it is a distinct field of $\mathrm{AI}$ research. In one sense it is a field of (pure) machine learning. A case-based reasoning system can be a program 
which operates unsupervised, and which automatically updates its own knowledge-base. CBR is distinct from the 'learning by example' model, in which a teacher contributes a significant amount of guidance to the system as it develops its knowledge-base. Yet there is a common principle, that knowledge can take the form of exemplars, or precedents, and the system can learn by these. Somewhere in between these two extremes lies incremental concept formation, with a model such as UNIMEM. Here the system is given a set of examples with which to form its own knowledge, but the system does have a degree of supervision. A question arising from this comparison is, "does the autonomous nature of a system relate to its level of artificial intelligence?" In one sense the answer to this question could be "yes". One could view the stand-alone CBL program, C-UNIMEM, as a more artificially intelligent program than the CBR assistant model described in this chapter. However, the application of C-UNIMEM was tested under laboratory conditions. That is to say, whilst dysmorphology presents a weak medical domain theory, the conditions of the experiments described in Chapters 4, 5 and 6, present a relatively closed, strong domain. These conditions may be carried through to the broader, realworld domain of dysmorphology, but only with respect to localised syndrome groups. Thus, there has been some trade-off in terms of the level of artificial intelligence with the CBR assistant model, but this has been necessary in attempting to present a practical solution.

\subsection{Summary}

- The practical theme of the work has resulted in a CBR assistant model which incorporates the work discussed thus far by the thesis along with some additional utilities such as a ranking function, and greater user interaction.

- The ranking formula utilises the contrast model and can be applied exhaustively against each syndrome in order to create a differential diagnosis, or used against a selected group for comparative similarity scores. Due to the non-uniformity of case descriptions, a ranking function can sometimes fail to list the correct diagnosis within the differential. A complementary procedure involving a standard indexing mechanism can alleviate this problem. 
- Case-based learning can have two modes: as a stand-alone program such as CUNIMEM, or as an interactive program under expert supervision. The stand-alone CBL program is impractical with regard to the broad syndrome nomenclature as it continually updates the case memory. However, it still may provide a useful analytical tool. A second mode of case-based learning uses a generalised version of the program within the context of the CBR assistant model. This program stops short of actually editing the case-base, and suggests reorganisations to the expert rather than automatically performing them.

- Additional user-oriented functionality has been developed to account for the greater interactive contribution of the user with the CBR assistant model. This comprises case-base editing functions, and utilities which allow the diagnostic model to be altered according to the users own interpretation of feature salience. 


\section{Chapter 8}

\section{Conclusions and Future Work}

\subsection{Introduction}

The scope of any symbolic computer program operating in a complex medical domain should be viewed with regard to realistic limitations. That is to say, a diagnosis made by a computer should only be regarded as a suggestion that can be further explored by a physician, rather than a definite solution. A complex domain, in this sense, refers to a field which is weak, i.e., a domain in which knowledge is both incomplete and uncertain. Added to this, diagnosis and research within the dysmorphology domain relies to a great extent on non-symbolic investigation (clinical examination, diagnostic imaging, chromosome analysis, biochemical testing etc.). Nevertheless, numerous computer applications have been developed for the dysmorphology domain, and there has been wide recognition that a computer program (be it a database or expert system) can be a useful asset in diagnosis.

The aim of this work has been to take the explicit scope of a computer system in dysmorphology a step further, by addressing the task of research which involves analysis of the current syndrome nomenclature with regard to unknown disorders. This is a goal of learning in which the recognition of new syndromes (which could involve a reorganisation of what was previously thought to be correct) promotes a greater understanding of the domain. The mechanics of the learning task are modelled by case-based reasoning, a field of artificial intelligence (and more specifically machine learning). This approach has been adopted with an appreciation of the following factors:

- Case-based reasoning can be used to model both of the key performance tasks in dysmorphology: diagnosis and learning. 
- Whilst the major thrust of the thesis (i.e., the contribution to research) has a focus on case-based learning, this work has a background objective of providing a practical solution. Thus, the primary task of diagnosis is not ignored, and is considered an integral part of the system design.

Along with these objectives, the thesis addresses other important issues with respect to a computer application in dysmorphology, and to specific topics within case-based reasoning. A problematical aspect of the dysmorphology domain is the sparseness and non-uniform nature of patient data. Thus, a generic architecture which can account for such diversity is very much a beneficial aspect to a computer system in this domain. Whilst this aspect of the case-based system design has been motivated by practical issues, it has also provided an independent research focus with regard to case representation in CBR. A discussion of case representation issues with respect to CBR is given in Section 8.3.2. The work specific to the development of a case-based learning program (described in Chapters 4 through 6), which involved the application and extension of the contrast model of similarity assessment, has also provided a separate research focus. Whilst this work was driven by an objective of improving the performance of the casebased learning program, it has promoted some thought to similarity assessment as an independent research topic in CBR. The open issues of similarity assessment with respect to CBR, and with reference to the work reported in this thesis, are discussed at the end of Chapter 6.

\subsection{Relationship to other work}

\subsubsection{Computer Systems in Dysmorphology}

Chapter 2 gives a comprehensive review of computer applications within dysmorphology. There is no strict definition for what constitutes a case-based reasoning system, merely a fundamental principle that past (solved) cases, or exemplars, provide the context for solving new, similar problems. Thus, with this loose definition of CBR, all of the previous computer applications in dysmorphology could be described as an elementary form of CBR. After all, every system reviewed in Chapter 2 stores within its computer memory some form of prototypical case which is used for comparison with the presented case. However, case-based reasoning as a research field involves a number of inter-related issues such as memory organisation, (efficient) indexing methods, case representation, similarity 
assessment, case adaptation and so on. Previous computer applications in dysmorphology have not addressed most of these issues because they are not research projects in CBR (although each system implicitly addresses the topics of case representation and memory organisation). However, some dysmorphology systems do have a wider research focus with regard to computer techniques. For example, Schorderet and Weiner have focussed on Bayesian techniques for diagnosis, and more recently Keravnou et al. (SDD) and Veloso et al. (DYSMOR) have directed and reported their work as much to the AI community as to the medical world. Some of the other systems reviewed in Chapter 2 have a more practical goal. Systems such as LDDB, SDR, POSSUM, BDIS and OMIM have specifically aimed to provide useful and accessible tools for diagnosis of dysmorphic syndromes. The development of these systems has not provided a specific research focus in computer science.

In determining the relationship between this work and other dysmorphology computer systems the main issue is learning. Most other dysmorphology systems are purely diagnostic systems which store known syndrome prototypes within their computer memory. Two systems specifically have a learning goal: LDDB and SDR. Both of these databases are designed to store syndromes, diagnosed cases and undiagnosed cases. With each system, there is no computer program that analyses undiagnosed cases. Recognition of links between cases is a human function, and to assist this procedure LDDB and SDR rely to a great extent on their completeness and widespread distribution. The work described in this thesis differs from previous applications of computer science in dysmorphology in that it has specifically tried to automate the learning process (through CBR and dynamic case memory reorganisation). The practical theme of the work has driven the design of the case-based assistant model nearer towards some of the previous work, however. An indexing mode similar to LDDB has been developed (but based on a distributed architecture), and some experimentation with a ranking formula have been performed not unlike the Schorderet and Weiner approaches.

\subsubsection{CBR Models in Medicine}

A natural attraction for applying CBR techniques to medical fields is the belief that experience plays an important role in problem solving (diagnosis). More specifically experience can be represented by exemplars which can provide a guide for later diagnostic decisions. Kolodner presented a CBR framework for clinical problem solving using a self- 
organising memory and applied it to the psychiatry domain [45]. Here both the diagnostic task (classification, or problem understanding) and treatment (a planning problem) are aided by comparison with exemplars from memory. The CASEY system, developed by Koton ([48]), employs causal reasoning within its CBR model to diagnose patients with heart problems. CASEY employs a case representation in which case symptoms are explained by causal inference links between related symptoms. During the retrieval stage a causal reasoning knowledge-base is employed to explain feature differences between a new case and a retrieved case. If a positive match is justified by the reasoning process, the solution (which is a causal explanation) from the precedent is adapted to fit the new case. The MEDIC program, developed by Turner, applies a variation of CBR called schemabased reasoning to the domain of pulmonology [76]. Here the schema-memory organises diagnostic category information along with procedural knowledge, or plans, which are intended to guide the diagnostic process. Matching involves classifying a new case with respect to the category structures in memory, which in turn point to diagnostic plans which aim to provide an interpretation or explanation of a particular finding through a consultation with the user. Another problem solving CBR system is ROENTGEN, developed by Berger, which uses past plans of radiation therapy for cancer in the thorax, and transforms along certain plan variables (e.g., the angle and intensity of the beam) to adapt the plan of treatment to a current patient [11].

A common thread of these systems is that they involve a form of problem solving technique to arrive at an adapted solution. Dysmorphology, on the other hand, promotes a classification style of CBR. There is no requirement here to adapt or change previous examples to fit the current situation. Diagnosis is, in this instance, facilitated by classification. Furthermore, in non of these systems is learning is an explicit aspect of the CBR model, as it is with the model described in this thesis.

Another important distinction between this and previous applications of CBR in medicine concerns the choice of domain. The above systems represent some of the early applications of CBR to medicine (and some of the foundational work in CBR). The specific fields of medicine to which they pertain are relatively closed. That is to say, they are medical domains in which knowledge is complete, or which have a strong domain theory. Conversely, dysmorphology represents a weak theory domain. A tenet of this thesis is that the standard CBR model is now sufficiently well-defined in order to examine its applicability to such weak domains. Perhaps therefore, the system with which this work 
has most resemblance is the PROTOS system, developed by Bariess and Porter $[9,56]$. The PROTOS system has been designed specifically as a model for weak theory domains in which exact case descriptions are favoured over a generalised memory structure (which is discussed below with regard to case representation issues). It should also be noted, however, that the PROTOS system has been designed as a generic model rather than specifically to a medical field ${ }^{1}$.

\subsection{Contributions to Research}

\subsubsection{Contribution to Dysmorphology}

\section{Automated Learning}

Of the many computer systems dedicated to some aspect of dysmorphology, none has specifically attempted to automate the research oriented learning involved with the recognition of new syndromes. The work described in this thesis has addressed this aspect of dysmorphology.

The main thrust of the work with regard to the dysmorphology field has been to automate the learning role of a computer application. This goal has been achieved with respect to two views: as an independent learning program, and as a facility within the context of a general diagnosis and learning system. Chapters 4 through 6 report on the former of these two views. They describe a case-based learning program which, with sufficient data, can be used as a stand-alone experiment with regard to grouping cases with multiple malformations. This type of program will be of use to the dysmorphology community provided that a sufficiently large repository of patient data (perhaps with diagnosed as well as undiagnosed cases) is compiled. The LDDB and SDR systems have this objective. The second view of the application of CBL to dysmorphology is described in Chapter 7. Automated learning is seen as an interactive process in the context of a general CBR assistant model which comprises an architecture which can be reorganised, but under user control.

\footnotetext{
${ }^{1}$ The literature describing PROTOS refers to an application to audiology along with more abstract domains.
} 


\section{Architecture}

A generic case-based architecture has been developed which allows the integration of different representations of case data within one case-based system. This is an important feature with respect to dysmorphology due to the non-uniformity of data originating from different sources.

One of the main points to arise from the review of computer applications in dysmorphology in Chapter 2 concerned the variability, or non-uniformity, of case and syndrome descriptions from different sources. This aspect is accentuated also by the intended scope of different computer applications. For instance, whereas LDDB accounts for a wide range of syndromes and employs a general case representation comprising clinical and radiological components, systems such as SDD and SDR, which account for a much smaller, specific syndrome group, employ a more specific case representation (i.e., based primarily on the skeleton). Another influential aspect of previous computer systems in dysmorphology regarding this matter has been the isolated way in which they have been developed. This has only added to the non-uniformity of descriptions, or representation. At the current time it is unclear whether or not this problem will be resolved by a standardisation of the nomenclature (and perhaps by one of the more recent and comprehensive systems such as LDDB, SDR or POSSUM becoming an established world-wide repository for case records). The work presented here has addressed this issue through the design of a generic architecture with regard to case nomenclature. The architectural design described in Chapter 3 is such that cases and syndrome with comprising different anatomical representations can utilise the same underlying functionality, and hence the same top-level procedural programs (i.e., the CBL and object retrieval algorithms).

\section{Similarity Assessment}

Practical considerations with respect to the goals of the research have resulted in the adoption of a compromise approach to similarity assessment which involves the utilisation of a mathematical formula. Previous work has relied on comprehensive statistical data. This work is original in employing the 'contrast model' with a model of feature salience.

The computer applications described as ranking systems in Chapter 2 have attempted a compromise to automated intelligence with regard to diagnosis. They have employed a readily available mathematical model in order to approximate the diagnostic process in 
terms of a comparison of dysmorphic features. The trend has been, in these instances, to use a statistical model, specifically Bayes's formula or a derivative there of. Both Chapters 2 and 6 highlighted the unsatisfactory aspects of the Bayes' model to the incomplete medical domain of dysmorphology. A key problem is its reliance on sufficient statistical information. The major ramification of this is that Bayesian formulae can only be employed with the more common syndromes.

This work has introduced the application of Tversky's contrast model for matching dysmorphic syndromes with cases of multiple malformations. The advantage that the contrast model has over Bayesian formulae is that instead of relying on statistical information across the syndrome group, it relies on the existence of a comprehensive nomenclature for dysmorphic features and an associated indication of individual feature salience. Fortunately, these two requirements are met by the LDDB feature listing used throughout this work. However, it should be noted that the diagnostic levels which have been used in this instance as an indication of salience are not exact definitions of diagnosticity, and have been derived in order to allow efficient indexing with LDDB.

\subsubsection{Contribution to Case-Based Reasoning}

The major functions of a case-based reasoning system interact and it is sometimes difficult, therefore, to isolate each functional unit as an independent research topic. This facet of CBR is certainly true with regard to the work described in this thesis. The research focus of case-based learning naturally requires the topics of similarity assessment and case memory organisation to be addressed, and the organisation of the case memory can in turn influence the case representation. With this in mind, this section presents the main contributions of this work with respect to specific areas of CBR research.

\section{Case-Based Learning}

$A$ major feature of the work, with regards to $C B R$, has been the development of a casebased learning function designed to operate with real data from the dysmorphology domain, This also provides a generic model of CBL for domains which rely on feature salience rather than a statistical measure of similarity.

Chapter 6 concludes with a model of case-based learning which initially started life as a discriminative, indexing algorithm, i.e., UNIMEM. The main extension to the UNIMEM 
model has been with regard to its matching operators. Whereas UNIMEM compares entities merely by counting the number of overlapping features, the W-UNIMEM and C-UNIMEM programs do specifically incorporate a model of similarity assessment, the former still based on a feature count, and the latter consisting of a slightly extended version of the contrast model. A key aspect to the UNIMEM model with regard to the dysmorphology domain is that its matching is polymorphic, i.e., two entities do not have to match on all features. The W-UNIMEM and C-UNIMEM models both retain polymorphic matching and the C-UNIMEM program extends this effect by accounting for discriminative features. Furthermore, the contrast model employed by the C-UNIMEM program allows user bias to play a part in the matching performed by the algorithm.

Whilst the application of the contrast model is of particular relevance to the dysmorphology domain, its initial definition is as a generic model for similarity assessment [77]. Its incorporation within the similarity phase of a generic case-based reasoning model is feasible provided a feature nomenclature exists (which is comprehensive across case descriptions) with an associated model of feature salience. A full critique of the case-based learning model, C-UNIMEM, is given in section 6.4 of Chapter 6.

\section{Case-Based Architecture}

Whilst the development of a generic system has been due partly to the non-uniform nature of available data which originates from different sources, this aspect of the work should prove a valuable contribution to research in $C B R$, particularly when applied to domains in which the representation of cases can vary according to interpretation.

It has already been noted that the generic case-based architecture described in Chapter 3 has beneficial implications with specific regard to the dysmorphology domain. This aspect of the case-based system design also presents a new focus to general CBR theory. It is only over the past few years that CBR research has moved away from the laboratory to real-world application domains (although much research still utilises laboratory data). This perhaps explains why there has been no issue as such with regard to a generic CBR architecture that can operate on cases with different representations. Data has been limited to cases with well defined representations from within one specific domain. This is to be expected as much of the emphasis on research has been with regard to the general CBR model and its associated elements. Furthermore, one of the distinctions of CBR 
from the broader AI field of reasoning by analogy is that it is fixed within one contextual field [31]. Nevertheless, interpretation and descriptions of cases can vary within one contextual field (as it does in dysmorphology).

Another key issue with regard to the case-based architecture described in this thesis concerns dynamic learning. Memory organisation is crucial to a dynamic memory system that reorganises its category structure, and for the most part a hierarchical architecture has been adopted. However, with this work it has been necessary to design a general architecture that can account for non-hierarchical operations as well as enabling localised hierarchical structures for CBL. This is due to the weak nature of the field, which at a higher, more abstract level is hierarchical (i.e., the general syndrome nomenclature), but the uncertainties and sparseness of data within the field (along with the fact that many syndromes stand isolated, or at one category level, thus causing a very wide and shallow hierarchy) make it difficult to develop an efficient, practical memory structure based on one hierarchy.

\section{Case Representation}

Along with specific developmental contributions to research in dysmorphology and CBR, this research has allowed an insight into open issues of case-based reasoning, particularly with regard to similarity assessment and case representation.

As with similarity assessment, case representation is considered by the CBR community as one of the fundamental components of a case-based system. Consequently, as with similarity assessment, case representation also has a number of associated open issues with regard to its place as an independent research topic. A number of open questions were put forward by the discussion group on case representation at the second $D A R P A$ case-based reasoning workshop [24]. A couple of these are relevant to the work that is reported in this thesis. Firstly, to what extent should cases be generalised as they are stored? And what arguments are there for maintaining the distinctness of cases that are apparently very similar? There are two extremities in approach with respect to this issue. A discrimination algorithm such as UNIMEM demonstrates one of these. With UNIMEM the generalisation process is total and continual. It is total in the sense that individual cases are not stored in terms of their overall description, only their distinctive features are stored. In this instance, memory consists 
only of generalised cases, or prototypes, and sets of distinctive features that provide exceptions to the general case, not actual cases. The PROTOS system $[9,56]$ demonstrates the other extremity in approach. The PROTOS system is designed specifically for weak domains in which knowledge is incomplete and poorly understood such that definitive rules are difficult to derive. The main tenet of the work involved with the design of PROTOS is that in weak domains 'exact' matching should be favoured over the use of generalised cases. That is to say, it is better to store one very prototypical, actual case, rather than a generalised amalgam of a related group. With respect to these two views on generalisation in memory, dysmorphology promotes an architecture that lies somewhere in between. Certainly dysmorphology is a weak theory domain, however, the whole field revolves around generalised prototypes. At the same time, it has been shown that the discriminative approach, in which cases may actually be stored more than once and in terms of different feature sets (which is not a complete case description) is not intuitively correct. Comparison with actual past cases will always be necessitated in learning new syndromes. The distributed architecture for case memory described in Chapter 3 is a compromise. It stores both generalised prototypes and actual cases together. The fact that they are both stored in terms of the same representation is important. It dictates that a large proportion of the utility software can be applied regardless of the type of object.

A second question concerns the physical storage of cases. Are cases monolithic structures that are applied individually, or are they loosely connected sets of events that are reconstructed at retrieval time? The solution to this will depend on the relative importance to the CBR system of retrieval and indexing procedures. If the CBR system adopts a flat database as its memory structure, such that search will not be confined, then a monolithic representation will suffice. Both LDDB and SDD employ a monolithic representation without confined search. However, if search is to be confined through a hierarchical memory organisation, then a monolithic case representation can cause problems if there is non-uniformity between cases. This problem is evident in dysmorphology. Individual cases may be recorded in terms of many, or very few features. If a case is recorded in terms of only a couple of significant abnormalities, and search is a matter of a sorting downwards through memory in conjunction with a similarity assessment function, then a case with few features might not achieve the necessary similarity to be retrieved (this was demonstrated by the example of Case $\mathbf{5 7 5 4}$ 
in Section 5.4 of Chapter 5). Thus, when cases are very non-uniform a weighting model such as that used by W-UNIMEM and C-UNIMEM will be necessary. However, there is also the additional problem that has been noted regarding the ordering of cases. In hierarchical memory, a case is stored below a general prototype. If search is only topdown, then the case can be effectively hidden by the prototype, i.e., in order to reach the individual case the search probe must first evaluate successfully with the prototype. If, however, the individual case has a particularly distinctive feature that is not contained within the generalised prototype description, then search may fail to retrieve this case even if the distinctive feature is part of the search probe (or index). The distributed case representation, memory organisation and the indexing method that has been described in this thesis address these two problems. Because search is not necessarily top down, and can be initiated at a relevant localised storage point, search will always find matching cases. Furthermore, search is confined.

The dysmorphology domain promotes another issue regarding case representation which has not been specifically noted in the CBR literature. That is the necessity to account for different representations of a case within one case-based architecture. The problem goes beyond the variation in interpretation of specific dysmorphic anomalies. In dysmorphology, the actual structure of case representations vary according to the higher order category as well as the interpretation of the physicians that compile the case records. The representation schema will depend very much on the scope of the syndrome database. The difference between the SDR and LDDB representations have already been shown to correspond to a skeletal specific representation against a more general clinical overview. SDD also has a skeletal specific representation, but this differs to that of SDR. An important point about these applications, which became apparent when researching them, is that they have been developed very much in isolation. There has been some collaboration between researchers of LDDB, POSSUM and GENDIAG in exchanging data. However, there has been no significant effort attributed to a unifying design for a syndrome database. It is unclear whether or not this would actually happen. More likely, a few systems will gradually become the established syndrome databases (LDDB, POSSUM and perhaps SDR; these are the most comprehensive at the current time). If this happens there will probably be an adherence to the nomenclatures that have been developed for these systems. 


\subsection{Critical Analysis}

\subsubsection{Case-Based Learning}

The case-based learning experiments reported in Chapters 4 through 6 utilise four data sets of cases that correspond to known disorders. Furthermore, each set comprises cases of a number of different but related conditions. It was noted in Chapter 6 that such data provides a very artificial environment with regard to the presentation of individual cases over such a short time period and the fact that each case in the set belongs to a higher order group. The structure of each data set has been specifically constructed in order to test the performance of the respective learning program. If each data set comprised a number of non-related cases along with related cases, the only difference to the resulting category structures would be a larger number of unclassified instances (stored at the root).

For the work involving case-based learning it was necessary to attain known case data in order to assess program performance. It is important to note, therefore, that the availability of data within dysmorphology in general is restricted, and there are a couple of reasons for this. There is sometimes a reluctance within the field to exchange case data, particularly concerning rare, or undiagnosed cases. It should be remembered that dysmorphology is a field of medical research as well as clinical practice. For this reason the recognition of a new condition carries an additional importance to individual researchers in that there is an inevitable source of publication material. This factor introduces an unfortunate element of competition within the field. Another problem is that much of the specific case data is not stored in a computerised form. For instance, with LDDB diagnosed cases are not comprehensively stored within the system. LDDB stores syndrome prototypes and rare isolated cases. Diagnosed cases are not stored because they are not considered necessary when the system holds syndrome prototypes (for the main task of diagnosis). The developers of LDDB actively encourage the storage of undiagnosed cases in order to promote possible learning with respect to the undiagnosed cases as the system is updated and re-distributed. Diagnosed case data does, of course, exist with respect to the LDDB system, but not necessarily in the LDDB computer format ${ }^{2}$

As a consequence of the artificial nature of experimental data, an important ques-

\footnotetext{
${ }^{2}$ The Acrocephalosyndactyly data set was transferred from medical records to the LDDB format especially for this research by Dr J. A. Maat-Kient.
} 
tion with regard to the work specific to case-based learning is "how do we know it will work with other data sets?" The CBL mechanism (most notably C-UNIMEM) has been demonstrated to work with symbolic data with a consistent feature nomenclature and an associated indication of individual feature salience. This thesis has made the assumption that cases of similar etiology, whether known or unknown, are similar phenotypes, i.e., they express a similar pattern of malformation. Thus, as the pattern of malformation is what is represented symbolically, the CBL program will work with data sets of cases of unknown etiology. However, similar phenotypes can have different etiologies, although they may initially be thought to be the same. In this situation the CBL program would fail, as would any computer program which utilised merely a symbolic representation of dysmorphic features. Further critical analysis of the work specific to case-based learning is documented in Chapters 4 through 6.

\subsubsection{The CBR Assistant Model}

A question posed at the end of Chapter 7 concerned the degree to which the general CBR assistant model is artificially intelligent. Compared to stand-alone programs, or non-interactive CBR systems which make all the relevant decisions, then such a model is presumably less intelligent. This weakening of the automated, or artificial, intelligence of the system has been necessary due to the limitations of data and the practical mapping of the CBR theory to the domain despite its intuitive appeal. The CBR assistant model is presented with regard to a broad spectrum of syndromes, and uses diagnostic and similarity models which also relate to the syndrome nomenclature as a whole. The intelligence of the CBR assistant may be increased (in the form of elicited and compiled domain knowledge), but with some trade-offs. The indexing model is meant to provide one generic point in the system that can facilitate domain knowledge interaction with the system. As for the diagnostic model and the inter-dependent similarity model, the more focussed the system is on a smaller group of syndromes then the greater scope for knowledge elicitation with respect to specific domain knowledge.

The evolution of 'case-based decision aiding' as a topic within general CBR research may represent the movement of CBR theory from the laboratory to the real-world. In domains where decisions can be crucial, and have major impact on later processing, then perhaps human decisions will always be necessary. This factor is accentuated when the computer system utilises purely symbolic data within a domain in which the relevant 
performance tasks also require analysis of additional information.

\subsection{Future Work}

The performance of any computer application involving artificial intelligence techniques in dysmorphology will be dependent on the depth of domain knowledge available to it. The fact that complete knowledge is actually unavailable to human experts dictates that there should be realistic aims with regard to computer systems in dysmorphology. The work described in this thesis, and in particular the functionality that involves calculating of a measure of similarity, has only used knowledge about dysmorphic features. For instance, it has not utilised information about chromosomes, family histories or biochemical data. An advantage of this approach is that a similarity model may be developed to operate across a broad spectrum of dysmorphic syndromes. The disadvantage is that the 'depth' of the similarity model will be restricted. There is certainly scope for additional factors such as chromosomal and biochemical data to be incorporated within a general similarity model, and this may improve the accuracy of matching. Another possibility, with regard to similarity assessment, is the inclusion of 'normal' features, which have not been utilised in this work, but certainly do participate in matching. Unfortunately, the acquisition of such detailed information appears as if it will remain a difficult task, particularly with regard to patient data.

An associated problem, with regard to developing a model of similarity, is that the range of disorders is great. Consequently, the acquisition of matching knowledge to cover all chromosomal and non-chromosomal syndromes, skeletal dysplasias and all the subsequent sub-categories of disorders, with respect to comprehensive details of each condition, is a major exercise in knowledge acquisition. In one sense, CBR can ease the knowledge acquisition problem, because knowledge can be transferred in terms of prototypical cases. This is useful if the design of the system dictates that knowledge is compiled within the representation of cases. In this way, there is further scope for compiling a greater depth of matching knowledge within the case representation. Along with biochemical and chromosomal data, perhaps knowledge of feature inter-dependencies (including normal and abnormal feature interactions), sex, status and temporal information ${ }^{3}$ could be linked

\footnotetext{
${ }^{3}$ The SDD project has involved considerable work in temporal matching, which is particularly relevant to disorders of the skeleton. With regard to the broader spectrum of syndromes, however, temporal development of organs and bones is not diagnostically significant for many syndromes.
} 
into the case and syndrome representation.

With regard to the models of case-based reasoning and learning that have been developed in this research, the inclusion of a more accurate similarity model will clearly have benefit. One of the features of the similarity model utilised in this work is that it essentially operates on two entities: a subject and a referent. One criticism of the discriminative case-based learning approach is that the ordering of cases affects the creation of category descriptions, and that individual cases can become hidden from the learning procedure (and the associated similarity function) behind the group prototype. Thus, when a category description is updated (through the evaluation procedure) the descriptions of individual cases do not participate in the assessment of similarity (which is only between the new case and the node description). This process seems intuitively incorrect, especially in a field in which syndromes can be based on a small cluster of individual cases. In such an instance, updating a syndrome description would involve matching the new case with both the syndrome description and the relevant diagnosed cases (retrospectively). Thus, in the real world the ordering of cases has a less detrimental effect on category information. A promising line of investigation is to incorporate a more general similarity model that operates with a group of entities rather than just one subject and referent. Such a model could provide a stand-alone function within the general CBR model, or take the form of a look-ahead, or retrospective, matching operator within the hierarchical case-based learning program. 


\section{Appendix A}

\section{Terms and Procedures in}

\section{Dysmorphology}

\section{A.1 Terms of Anatomical Development}

\section{A.1.1 Individual Alterations of Form or Structure}

Malformation: A malformation is a morphologic defect of an organ, part of an organ, or larger region of the body resulting from an intrinsically abnormal development process [70]. This definition has two important specifications: a malformation is an abnormality of an organ and it is intrinsic. Abnormalities of tissue development and their anatomic results are not malformations; they are dysplasias. Intrinsic means that the developmental potential of the organ, its anlage ${ }^{1}$, was abnormal. From fertilization the organ never had a chance to develop normal form or structure. The fact that an abnormality may not be recognizable at an early stage of development does not mean that the anlage is normal. If the anlage was normal and the defect resulted from a later, extrinsic interference of an initially normal developmental process the result is called a disruption. The term malformation is used for both the process and its morphologic result. Most, if not all malformations are field defects. A morphogenetic (or developmental) field is a region or part of an embryo which responds as a coordinated unit to embryonic interaction and results in complex or multiple anatomic structures. Embryonic interaction refers to the reciprocal (chemical or physical) influence of one developing tissue on another (or

\footnotetext{
${ }^{1}$ The first accumulation of cells in an embryo that constitutes the beginning of a future tissue, organ, or part.
} 
more than one other) during embryogenesis. An intrinsic, nondisruptive disturbance of a developmental field will lead to a field defect manifested as multiple anomalies.

Disruption: A disruption is a morphologic defect of an organ, part of an organ, or larger region of the body resulting from the extrinsic breakdown, or an interference with, an originally normal developmental process. In contrast to a malformation, the developmental potential of the involved organ was originally normal. Secondarily, an extrinsic factor such as an infection, teratogen ${ }^{2}$, or trauma interfered with the development which thereafter proceeded abnormally.

Deformation: A deformation is an abnormal form, shape, or position of a part of the body caused by mechanical forces. As with the term malformation, the term deformation is used for both the deforming process and its result. The use of the term deformation is restricted to aberrations of form considered a normal response (of the affected tissue) to unusual mechanical forces. These forces may be extrinsic to the fetus or intrinsic. For example, a prenatal deformation is equinovarus foot ${ }^{3}$, which may result from extrinsic compression of the fetus caused by oligohydramnios ${ }^{4}$. A similar foot condition may also result from an intrinsic abnormality, such as meningomyelocele ${ }^{5}$, with a resulting deficit of foot movement and hence foot deformation. Correction or release of the deforming mechanical force would lead to normalisation of the developmental process.

Dysplasia: A dysplasia is an abnormal organisation of cells into tissue(s) and its morphologic result(s). In other words: a dysplasia is the process (and consequence) of dyshistogenesis ${ }^{6}$. The term dysplasia applies to all abnormalities of histogenesis. In contrast to malformations, disruptions and deformities, dysplasias are not confined to single organs. Dysplasias can be localised, in which case the abnormal tissue elements occupy part of an organ, and generalised, which can effect multiple organs.

\footnotetext{
${ }^{2}$ A teratogen refers to anything that causes teratogenesis, the development of abnormal structures in an embryo resulting in a severely deformed fetus.

${ }^{3} \mathrm{~A}$ form of clubfoot.

"Abnormally small amount of amniotic fluid

${ }^{5} \mathrm{~A}$ hernia of the spinal cord and membranes through a defect in the vertebral column.

${ }^{6}$ Errors in histogenesis, the development of differentiated tissues of the germ layer, i.e., the origin and development of tissue.
} 


\section{A.1.2 General Terminology}

In addition to the above terms, which refer to qualitative changes of morphogenesis, a number of terms are used to define more quantitative alterations. Thus, hypoplasia and hyperplasia refer to underdevelopment and overdevelopment of an organism, organ, or tissue resulting from a decreased or increased number of cells, respectively. Hypotrophy and hypertrophy refer to a decrease and increase in size of cells, tissue, or organ, respectively. The term agenesis connotes the absence of a part of the body caused by absent anlage, whereas in aplasia the absence of a part of the body results from a failure of the anlage to develop. The term atrophy is to be used when a normally developed mass of tissue(s) or organ(s) decrease because of a decrease in cell size and/or cell number.

\section{A.1.3 Patterns of Morphologic Defects}

Multiple anomalies in a given patient may be causally or pathogenetically related, or occur together on a statistical basis or by chance alone. Different terms are used to express the type of relationship, each refiecting the level of knowledge on the causation and genesis of the pattern.

Polytopic fleld defect: A polytopic field defect is a pattern of anomalies derived from the disturbance of a single developmental field. The disturbance of a developmental field may result in a localised anomaly (monotopic) or in a pattern of anomalies (polytopic). Dysmorphogenetically, these anomalies are derivatives of a single malformative or disruptive process.

Sequence: A sequence is a pattern of multiple anomalies derived from a single known or presumed prior anomaly or mechanical factor. A malformation, a disruption, or a mechanical factor may give rise to a cascade of secondary problems in subsequent morphogenesis. Thus, a myelomeningocele ${ }^{7}$ may lead to lower limb paralysis, muscle wasting, club feet, incontinence, urinary tract infection and renal damage, constipation, etc. This example is called the myelomeningocele sequence.

Syndrome: A syndrome is a pattern of multiple anomalies thought to be pathogenetically related and not known to represent a single sequence or a polytopic field defect. The use of the term syndrome implies a lower level of understanding of a given pattern of anomalies than polytopic field defect or sequence. In the former it is presumed that

\footnotetext{
${ }^{7}$ Spina bifida with portion of cord and membranes protruding.
} 


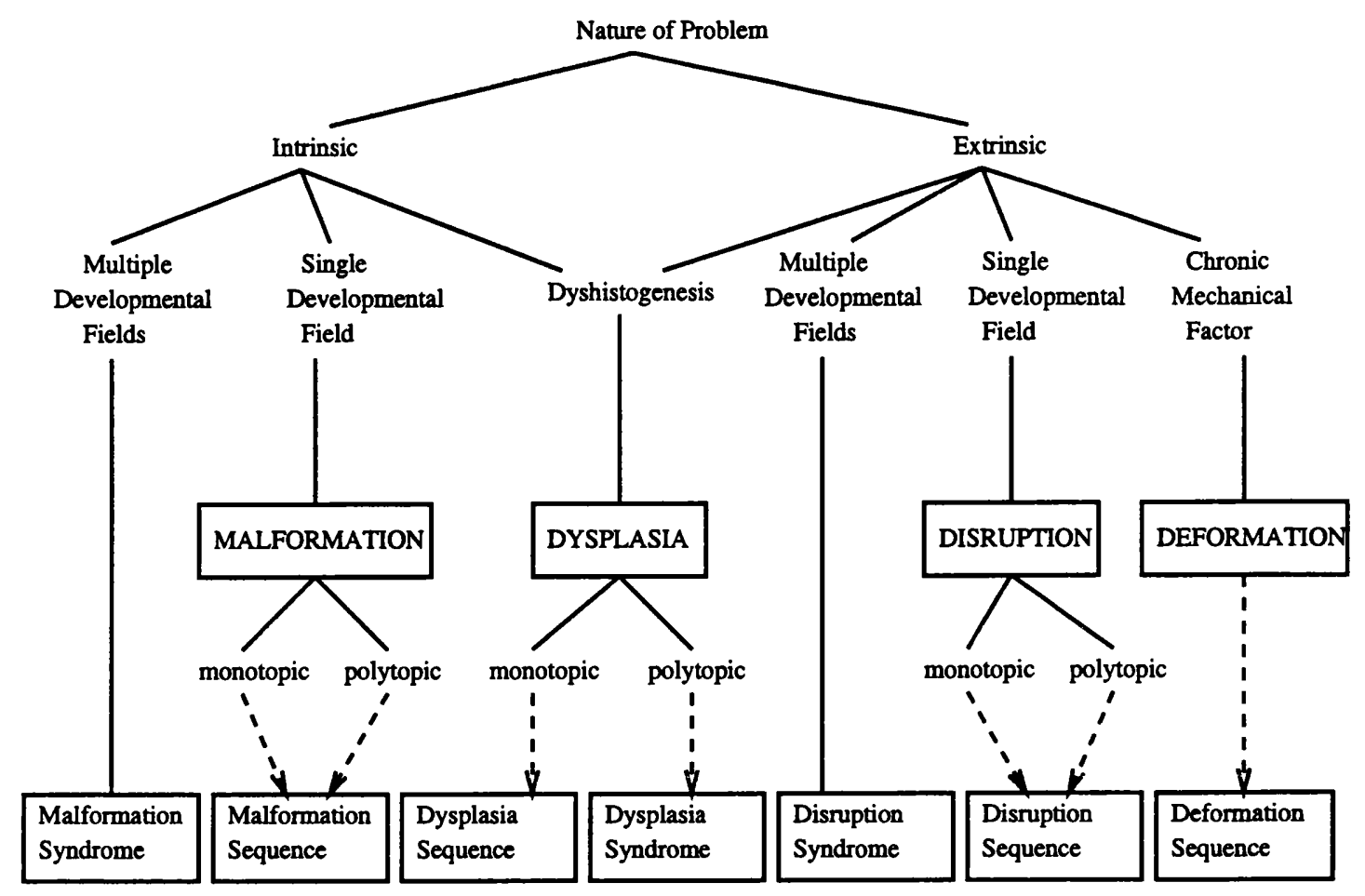

Figure A.1: Phenotype analysis in dysmorphology.

the anomalies are part of one and the same developmental field defect; in the latter the initiating event and the ensuing cascade of secondary effects are known. This knowledge is not available when the term syndrome is used. With increasing knowledge, a syndrome may turn out to represent a polytopic field defect or a sequence. Syndrome frequently implies a single cause, such as Down syndrome, but, as with sequence, the term is only used when the components of the given pattern of anomalies are known or thought to be pathogenetically related. The chance concurrence of two or more abnormalities and (statistical) associations do not constitute syndromes.

Association: An association is a nonrandom occurrence in two or more individuals of multiple anomalies not known to be a polytopic field defect, sequence, or syndrome. An association refers solely to statistically, not pathogenetically or causally related anomalies. With increasing knowledge a given association will ideally come to be broken apart into one or more sequences, syndromes or field defects. 


\section{A.2 General Principles of Dysmorphology}

Smith itemises the following important general principles and information that should be appreciated in the evaluation of a patient with multiple malformations [30].

\section{A.2.1 Nonspecificity of Individual Defects}

With rare exceptions, a clinical diagnosis of a pattern of malformation cannot be made on the basis of a single defect. Even a rare defect may be a feature in several syndromes of variant etiology. A specific diagnosis is usually dependent on recognition of the overall pattern of anomalies, and the detection of minor defects may be as helpful as that of major anomalies in this regard.

\section{A.2.2 Variance in Expression}

Variance in extent of abnormality among individuals with the same etiologic syndrome is a usual phenomenon. Except for such nonspecific general features such as mental deficiency (which occurs in over 600 syndromes) and small stature, it is unusual to find a given anomaly in 100 per cent of patients with the same etiologic syndrome. For example, in 21 trisomy Down syndrome, only mental deficiency is ubiquitous; hypotonia is a frequent feature, but most other individual clinical features are found in less than $\mathbf{8 0}$ per cent of such patients. A specific diagnosis of Down syndrome is generally based on the overall pattern of anomalies.

It is especially important to appreciate that the environmentally determined disorders occur in all gradations of severity. Also, intraindividual variability in expression is frequent, with variance in the degree of abnormality on the left versus the right side of the individual.

\section{A.2.3 Heterogeneity}

Similar phenotypes (overall physical similarity) may result from different etiologies. Only by finer discrimination of the phenotype or mode of etiology can such similar entities be distinguished. For example, Achondroplasia ${ }^{8}$ is frequently misdiagnosed among individ-

\footnotetext{
"A syndrome characterised by multiple exostoses (which are bony growths at the surface of a bone) of the epiphyses (or ossification centres of the bone), especially of the long bones, metacarpals and phalanges.
} 
uals who have chondrodystrophies ${ }^{9}$ that only superficially resemble true Achondroplasia [30]. A diagnosis should be rendered only when there is close resemblance in the overall pattern of malformation between the patient and the disorder under consideration.

\section{A.2.4 Etiology}

About a half of all known disorders are known to have a genetic basis [85]. Besides these, about forty per cent of individuals with multiple malformations have conditions that have not been recognised as specific disorders. A small number of such patients (approximately eight per cent) have a structural chromosomal abnormality. Genetic counseling is possible in these cases and is dependent on the hereditary nature of the chromosomal defect. In the absence of an evident chromosomal abnormality or familial data suggesting a particular mode of etiology, it is generally impossible to state any accurate risk of recurrence for unknown patterns of multiple malformation [30].

\section{A.3 Diagnostic Procedures and Investigations}

Winter and Baraitser outline the following investigations and procedures that are required for the diagnosis of dysmorphic syndromes [85]. This aggrees, for the most part, with a general overview given by Smith [30].

\section{A.3.1 History}

At least a three generation family history is necessary. In particular, information regarding consanguinity (blood relations), previous family members with similar conditions, causes of death in direct relatives, and details of any stillbirths or neonatal deaths should be sought. Following this, a pregnancy history should be taken. Smith agrees with these basic investigations, and puts a little more emphasis on fetal activity: vigor of fetal activity, getstational timing, indications of uterine contstraint, mode of delivery, size at birth etc.

\section{A.3.2 Basic Investigations}

- Chromosome Analysis is essential. The diagnostic process stops if an abnormality is found, but the dysmorphologist might not be satisfied with a normal result.

\footnotetext{
${ }^{9}$ Defects in the formation of cartilage at the epiphyses of long bones.
} 
Current chromosome analysis techniques may not be adequate and small deletions in specific chromosomes almost certainly go undetected. Clinicians should be naturally suspicious with regard to a normal karyotype. Knowledge of a sibling or parent chromosomal abnormality will direct the physician to specify a particular chromosome abnormality for careful investigation. The difficult task of syndrome recognition begins once a normal karyotype is established and accepted by the dysmorphologist.

- Biochemical tests can be important. Storage disorders are known to cause dysmorphic features, but disorders of amino acid and organic acid metabolism should also be considered.

- Diagnostic imaging is not routinely performed, although a number of dysmorphic syndromes cannot be diagnosed without a CT brain scan. Such a procedure usually results when the dysmorphologist already suspects a condition, in which a brain scan is performed as an additional investigation.

- Consultation with other specialists is an essential aspect of investigation. Many diagnostic features may be outside the competence of individual dysmorphologists to evaluate. For example, Aicardi syndrome does, to a large extent, depend on the recognition of punched-out areas in the retina and this might need an ophthalmological opinion.

- Clinical photographs are essential for clinical documentation, publication of important cases and consultation with colleagues. Photography is an important diagnostic procedure.

\section{A.3.3 The Diagnostic Procedure}

There are several ways in which a clinical diagnosis can be made. The first possibility is what Winter and Baraitser call gestalt recognition. There are some conditions that are instantly recognisable to an experienced physician, and do not require a detailed analysis of the dysmorphic features. For example, Williams syndrome (which comprises a number of facial defects regarding the eyes, nose and mouth and teeth) is considered a gestalt recognition syndrome [85] as the visual picture is distinct and a diagnosis might be instananeously made. However, all gestalt diagnoses must be checked, and features 
against the diagnosis should be considered.

If immediate recognition fails, the next step is to identify and define the specific dysmorphic features. Accurate assessment of features is essential. Smith puts forward the following three questions with regard to the interpretation of patient anomalies:

1. Which is the earliest anomaly?

2. Can all the anomalies in the patient be explained on the basis of a single problem that leads to a cascade of subsequent defects, i.e., a sequence?

3. Does the patient have multiple structural defects that cannot be based on a single initiating defect?

Where possible dysmorphic features should be quantified, for example, has the child got hypertelorism (eyes which are truly too widely spaced) or telecanthus (eyes which appear to be too widely spaced because of the laterally displaced inner canthal margins)? Are ears low-set, or are they posteriorly rotated giving the impression of being low-set [85]? Some specific anomalies might not be important with respect to a particular patient. The significance of an individual dysmorphic feature can change if the parents reveal that the specific anomaly is known to exist within the family, but for otherwise healthy family members. For example, if a child is mentally handicapped and has syndactyly involving toes 2 and 3 . The fact that the child has syndactyly may not be important if it is known that other members of the family with normal intelligence have this same feature. Another problem which should be considered is whether a dysmorphic feature in a parent occurring in isolation is a heterozygote ${ }^{10}$ manifestation or even an indication of somatic mosaicism ${ }^{11}$.

The diagnostic procedure, as described by Winter and Baraitser, involves the recognition and use of diagnostic handles. Handles refer to specific dysmorphic features that carry a certain level of diagnostic significance with respect to the different syndromes. That is to say, a good handle is a specific (diagnostically significant) dysmorphic feature that will point the physician to possible diagnoses for further investigation. In order to select a manageable list of candidates, good handles are not too frequent across dysmorphic

\footnotetext{
${ }^{10} \mathrm{~A}$ heterozygote refers to an individual who has different alleles for a given characteristic, where an allele refers to the form of a particular gene.

${ }^{11}$ Somatic mosaicism refers to a chromosome that has mutated during inheritance. More specifically, it concerns the fusion of two alleles from different chromosomes into one.
} 
syndrome. Thus, there is a trade off between establishing very significant features and features that are of relatively low incidence. This procedure is relevant to the use of the Winter and Baraitser computer system (the London Dysmorphology Database) and their syndrome compendium [85]. The following list is intended to guide the dysmorphologist in selecting good handles.

- Good handles are features that are easily recognisable as being abnormal and do not merge with normal variation.

- The severity of the malformation might turn a poor handle into a good one. For example, mild hypertelorism might not be a good handle whereas an interpupillary distance well above the normal centiles could be an important feature of the condition under consideration.

- A good handle is not common to many syndromes. Pre-axial polydactyly is a good handle as it does not occur in a large number of syndromes. For the same reason, mental retardation is not good handle as there are at least 600 different syndromes in which this occurs. However, mental retardation is such an important feature that it may be essential to the diagnosis of a specific condition.

- Good handles can change with age. For example, prominence of the lips is an important feature in the Coffin-Lowry syndrome, but this anomaly might not be present in the first 6 months of life and it might, therefore, be necessary to follow up patients to assess the evolving phenotype.

- Dysmorphic features may be primary or secondary phenomena. Consequently, when selecting good handles it may be necessary to investigate whether or not they are secondary phenomenon which might not constitute a main feature. 


\section{Appendix B}

\section{CLOS Constructs for LDDB}

\section{Representation of Eye Anomalies}

\section{B.1 Description}

The defclass construct defines the eyes object class according to the LDDB categories shown in Table 2.2. Each object has a unique identifier (id), and the specific case identifier to which the object belongs (case-id). Each attribute, or slot, has an associated generic access function, which in this instance is always the same alphanumeric string as the slot name. Also, each attribute can be initialised to a specific value through the initform argument.

When a new eyes object is created the relevant eye abnormalites are passed to the function make-eyes-frame. The list of specific eye anomalies is expanded to the required CLOS format and passed to a make-instance construct held within a Lisp macro form (the backquote mechanism is used to create the macro). For example, the list ((general hypertelorism) (iris coloboma)) will be expanded to (:general 'hypertelorism :iris 'coloboma). This revised structure is then spliced into the make-instance macro (the @ character denotes a splice operator). The macro is then evaluated in order to form the new eyes object. 


\section{B.2 Eyes Object Declaration}

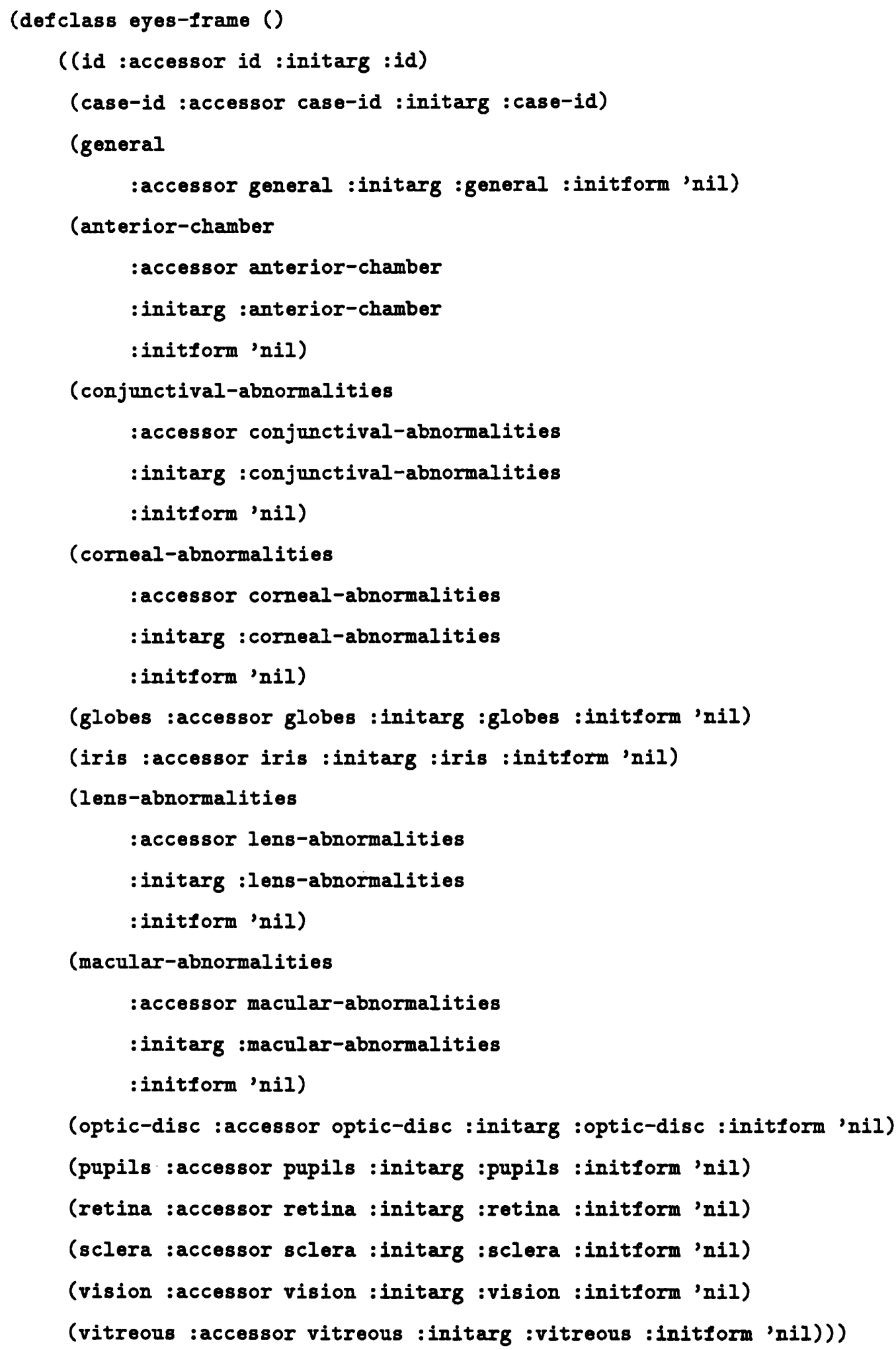




\section{B.3 Eyes Object Generation Macro}

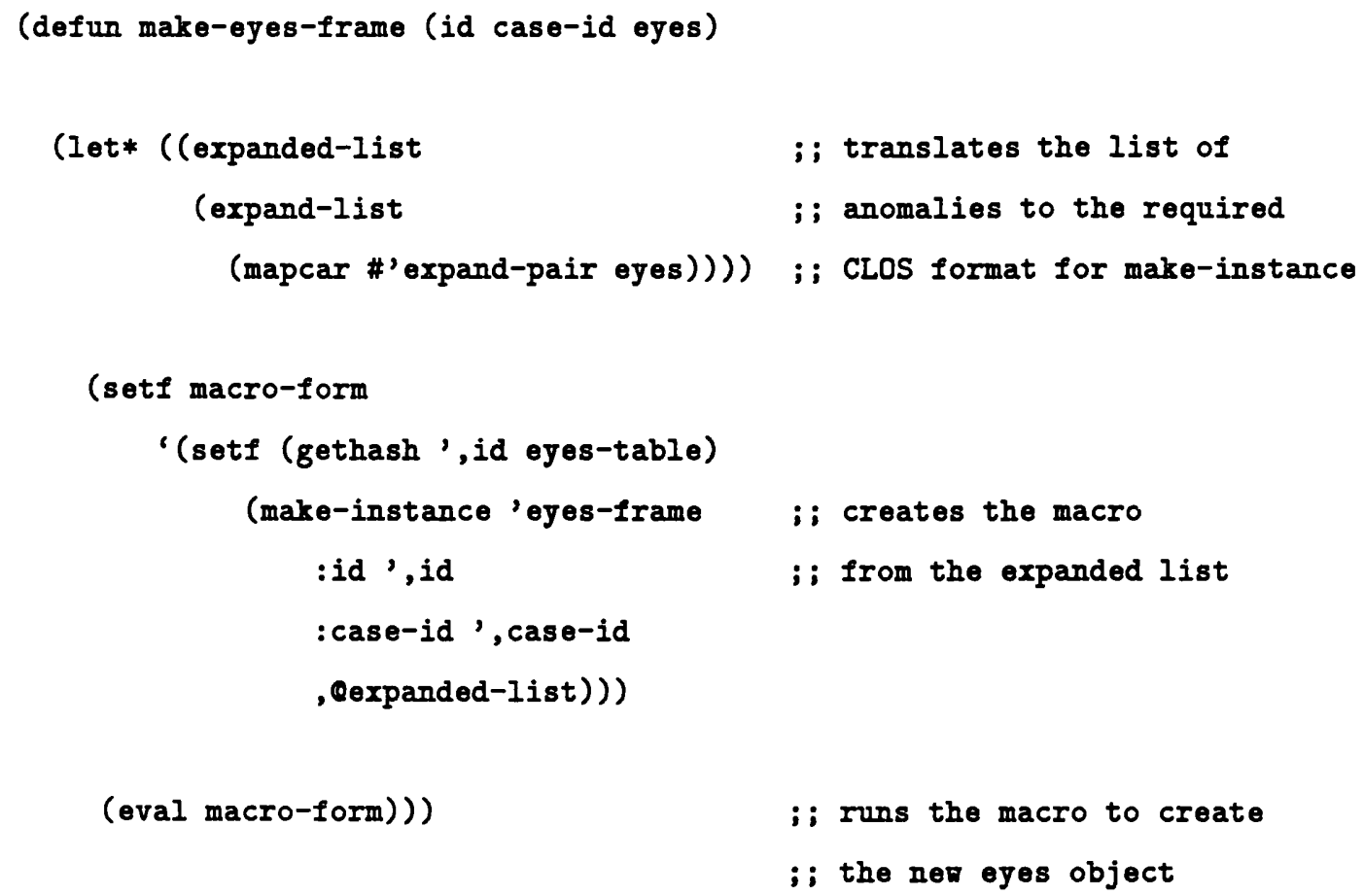




\section{Appendix C}

\section{Case Ordering Considerations for the C-UNIMEM Algorithm}

\section{C.1 Introduction}

For the purpose of analysing the performance of C-UNIMEM with alternative case orderings (as presented incrementally to the algorithm), three further experiments were performed with the Acrocephalosyndactyly data set. Table C.1 lists the original order of cases that was utilised in the experiments reported in Chapters 4 through 6 along with three further (random) case orderings that produced the three networks R.1, R.2 and R.3 shown by Figures C.1, C.2 and C.3 respectively. All parameter settings were kept the same as those for Network A.3 described in Chapter 6. Tables C.2, C.3 and C.4 list the node descriptions for each of the three Networks R.1, R.2 and R3 respectively.

\section{C.2 Discussion}

One consistent feature of all the networks generated by C-UNIMEM with the Acrocephalosyndactyly data set is that cases are always grouped according to the same syndrome family. This fact is reflected in the quantitative analysis provided by the mean group accuracy factor. Figure C.4 illustrates the comparative $\overline{G A F}$ 's for Networks A.3, R.1, R.2 and R.3. None of the new networks quite manage the $\overline{G A F}$ achieved in Network A.3 (+4.28), but certainly approach this score. Network R.1 achieves a $\overline{G A F}$ of +2.90, whilst Network R.2 has a $\overline{G A F}$ of +2.67 , and Network R.3 has the highest $\overline{G A F}$ of +3.40 . Clearly, the more case orderings that are utilised and compared with respect 


\begin{tabular}{|c|c|c|c|c|}
\hline Case Order & Networks A.1/A.2/A.3 & Network R.1 & Network R.2 & Network R.3 \\
\hline 1 & 8175 & 7312 & $5286 \mathrm{~b}$ & 0004 \\
\hline 2 & 6761 & 0004 & 6761 & 606067 \\
\hline 3 & 6638 & $5286 \mathrm{~b}$ & 5754 & 6351 \\
\hline 4 & 0001 & $5286 c$ & 0005 & 5754 \\
\hline 5 & 5462 & 5754 & 0001 & 564525 \\
\hline 6 & 616929 & 0005 & $5286 \mathrm{c}$ & 6566 \\
\hline 7 & 7464 & $5286 a$ & $5286 \mathrm{a}$ & 8967 \\
\hline 8 & 6351 & 8175 & 7385 & 9605 \\
\hline 9 & 618717 & $6150 a$ & 7312 & 6761 \\
\hline 10 & 0002 & 7385 & 7877 & 0005 \\
\hline 11 & 0003 & 8967 & 7464 & 7312 \\
\hline 12 & 0004 & 0003 & $6150 a$ & 7877 \\
\hline 13 & 6566 & 7877 & 606067 & 616929 \\
\hline 14 & 7031 & 7464 & 6638 & 0002 \\
\hline 15 & $6150 a$ & 606067 & 5462 & 7464 \\
\hline 16 & 7385 & 6638 & 6566 & 7385 \\
\hline 17 & 7877 & 0001 & 0002 & 4200 \\
\hline 18 & 0005 & 5462 & 0003 & 8175 \\
\hline 19 & 9605 & 6566 & 616929 & $6150 a$ \\
\hline 20 & $5286 a$ & 0002 & 9605 & $5286 \mathrm{c}$ \\
\hline 21 & $5286 \mathrm{~b}$ & 7031 & 8175 & 6638 \\
\hline 22 & $5286 c$ & 616929 & 564525 & $5286 \mathrm{~b}$ \\
\hline 23 & 4200 & 9605 & 6351 & 0001 \\
\hline 24 & 8967 & 564525 & 7031 & $5286 a$ \\
\hline 25 & 7312 & 6351 & 8967 & 7031 \\
\hline 26 & 5754 & 6761 & 618717 & 0003 \\
\hline 27 & 606067 & 618717 & 4200 & 5462 \\
\hline 28 & 564525 & 4200 & 0004 & 618717 \\
\hline
\end{tabular}

Table C.1: Four case orderings for Acrocephalosyndactyly data set.

to mean group accuracy factors would allow a more conclusive quantitative analysis, however, the additional three networks suggest a consistent level of group accuracy with regard to C-UNIMEM, the Acrocephalosyndactyly data set, and case ordering.

Figures C.1, C.2, C.3 and the associated description tables allow a more qualitative analysis with regard to case ordering. The general view appears to show some consistency with regards to the groups formed. Certainly, each network at least groups cases of the same syndrome family, and each network contains accurate groups pertaining to specific diagnoses. For instance, Node N8 of Network R.1 groups all five cases of Pfeiffer syndrome, as does Node N8 of Network R.2 and Node N4 of Network R.3. The associated node descriptions for these three groups listed in Tables C.2, C.3 and C.4 are 


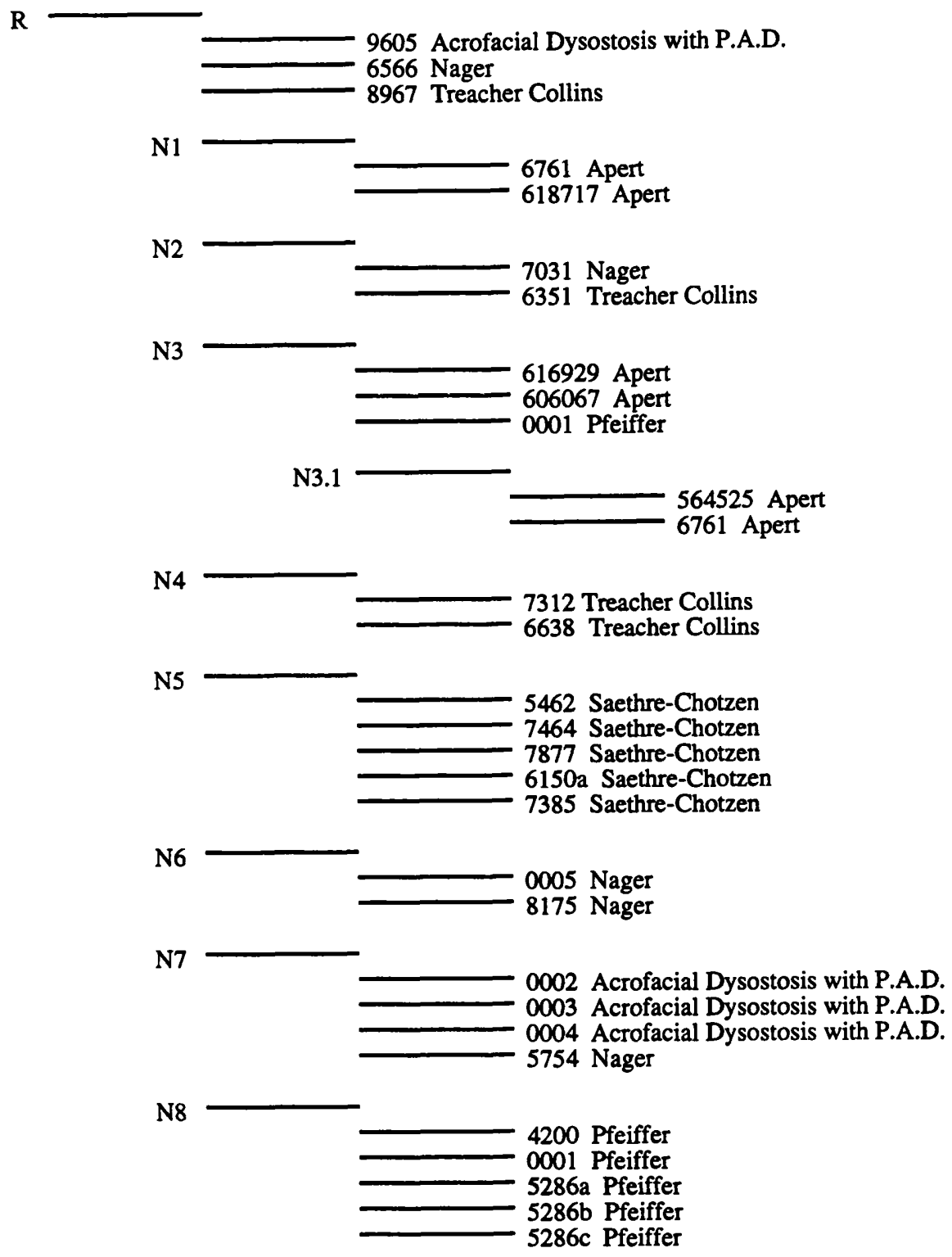

Figure C.1: Network R.1 


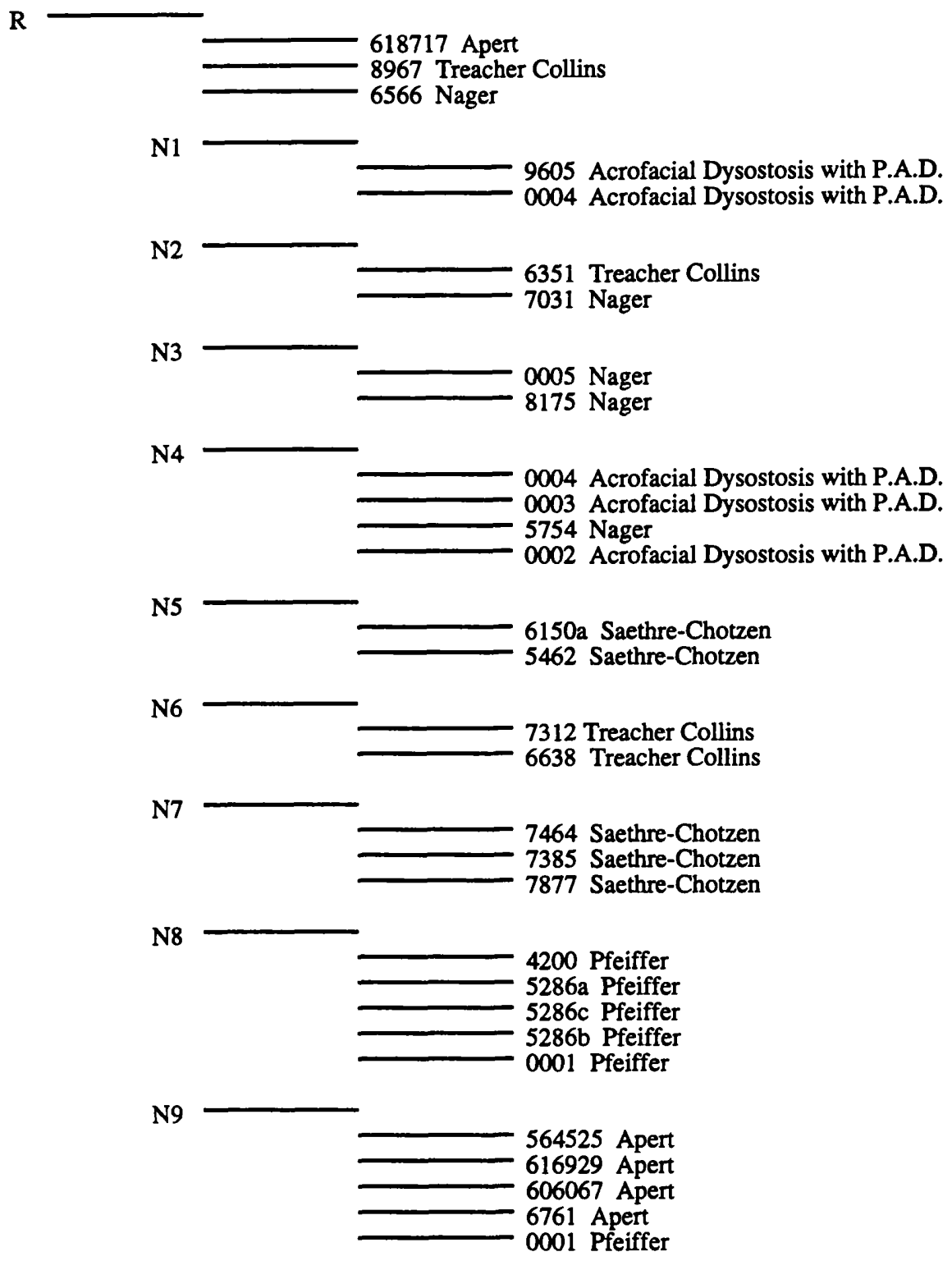

Figure C.2: Network R.2 


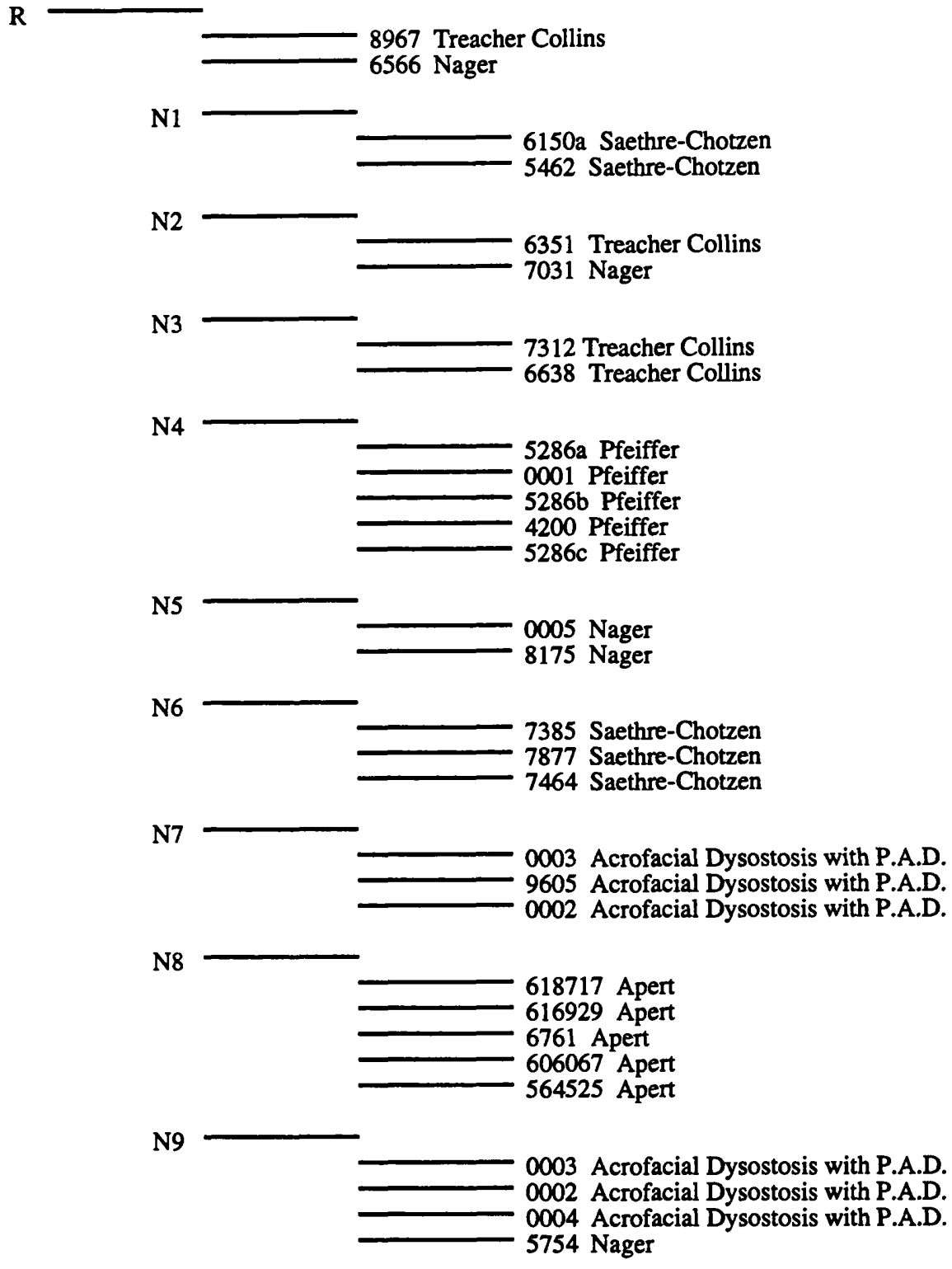

Figure C.3: Network R.3 


\begin{tabular}{|c|c|c|}
\hline Node & Permanent & Non-permanent \\
\hline N1 & & 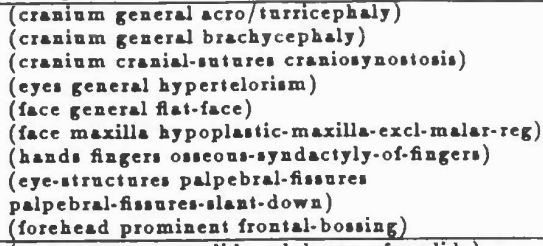 \\
\hline N2 & & $\begin{array}{l}\text { (eye-atractures eyelidi coloboma-of-eyelido) } \\
\text { (ears external-eari dyoplantic-earu) } \\
\text { (face malar-region flas-malar-region) } \\
\text { (eye-strnctures palpebral-fissures } \\
\text { palpebral-fissures-alant-down) } \\
\text { (face mandible small-mandible/microgathia) }\end{array}$ \\
\hline N3 & $\begin{array}{l}\text { (cranium cranial-sutures cranionynontonis) } \\
\text { (ejes general bypertelorism) } \\
\text { (ejes general prominent-eyen-including-proptosis) } \\
\text { (craning general acro/torricephaly) } \\
\end{array}$ & (forehead prominent frontal-boung) \\
\hline N3.1 & & 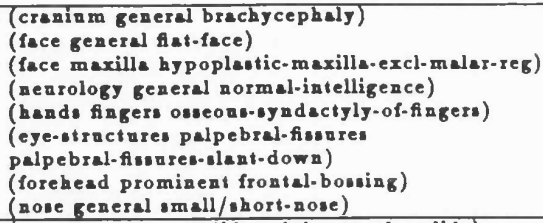 \\
\hline N4 & & $\begin{array}{l}\text { (eye-structures eyelids coloboma-of-eyelido) } \\
\text { (ears bearing deafness-non-opecific) } \\
\text { (nerrology general normal-intelligence) } \\
\text { (eye-structures palpebral-finares } \\
\text { palpebral-fisurea-olant-down) } \\
\text { (ears external-ears mall-earn) } \\
\text { (face mandible amall-mandible/micrognathia) }\end{array}$ \\
\hline N5 & $\begin{array}{l}\text { (ejes general hypertelorism) } \\
\text { (ears ear-crus prominent-ear-crus) } \\
\text { (cranium cranial-untures cranionyostosis) }\end{array}$ & (face maxilla hypoplatic-maxilla-exch-malar-reg) \\
\hline N6 & & 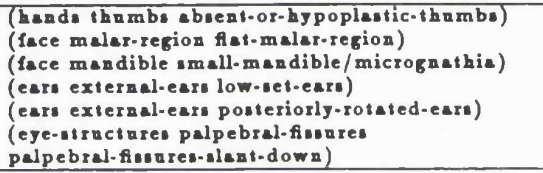 \\
\hline N7 & $\begin{array}{l}\text { (feet toes absent-toes) } \\
\text { (face mandible imall-mandible/micrognathia) } \\
\text { (hands fingers absent-fingers-or-oligodactyly) } \\
\text { (upper-limbs forearm hypoplastic-or-abient-ulna) }\end{array}$ & \\
\hline N8 & $\begin{array}{l}\text { (feet hallux broad-hallax) } \\
\text { (handi thambs broad-thambs) } \\
\text { (ejes general hypertelorism) } \\
\text { (forehead prominent frontal-bosaing) } \\
\text { (crenium cranial-sutures craniosynoutonis) }\end{array}$ & \\
\hline
\end{tabular}

Table C.2: Node descriptions for Network R.1.

identical ${ }^{1}$. Similarly, Node N5 of Network R.1, Node N7 of Network R.2 and Node N6 of Network R.3 all group cases of Saethre-Chotzen type Acrocephalosyndactyly and list the same dysmorphic features in their respective node descriptions. Cases of Acrofacial Dysostosis with Post-Axial Defects are also generally grouped together, with similar node descriptions (Node N7 of Network R.1, Node N4 of Network R.2, and Nodes N7 and N9 in Network R.3).

The specific effects of ordering on the C-UNIMEM algorithm require a more detailed scrutiny of the node descriptions. Such analysis has already been addressed, to some

\footnotetext{
${ }^{1}$ As would be expected, groups formed by the same two cases across the three networks have identical node descriptions.
} 


\begin{tabular}{|c|c|c|}
\hline Node & Permanent & Non-permanent \\
\hline N1 & & $\begin{array}{l}\text { (handi fingersebsent-fingers-or-oligodactgly) } \\
\text { (1eet toes abient-toes) } \\
\text { (oral-region palate cleft-palate) } \\
\text { (ears external-ears dysplastic-eara) } \\
\text { (face malar-region fat-malar-region) } \\
\text { (opper-limbs forearm hypoplastic-or-absent-nina) } \\
\text { (face mandible emall-mandible/microgathia) }\end{array}$ \\
\hline N2 & & $\begin{array}{l}\text { (ege-structures eyelids coloboma-of-eyelids) } \\
\text { (ears external-ears dysplastic-earu) } \\
\text { (face malar-region flat-malar-region) } \\
\text { (eye-structures palpebral-fisures } \\
\text { palpebral-fisures-blant-down) } \\
\text { (face madible small-mandible/microganthia) }\end{array}$ \\
\hline N3 & & 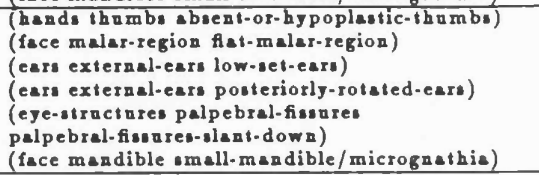 \\
\hline N4 & $\begin{array}{l}\text { (ieet toes absent-toet) } \\
\text { (handi fingers absent-fingers-or-oligodactyly) } \\
\text { (apper-limbs forearm hypoplastic-or-absent-ulna) } \\
\text { (face mandible omall-mandible/micrognathia) }\end{array}$ & \\
\hline N5 & & 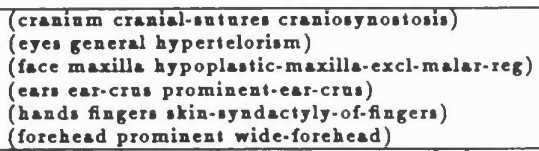 \\
\hline N6 & & 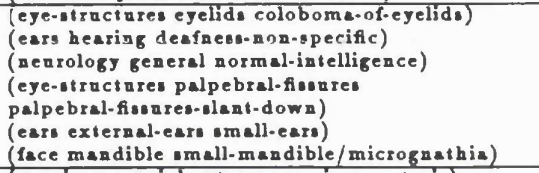 \\
\hline N7 & & $\begin{array}{l}\text { (craningm cranial-gutures craniosynostosis) } \\
\text { (eyes general hypertelorism) } \\
\text { (ears ear-crus prominent-ear-crus) }\end{array}$ \\
\hline N8 & 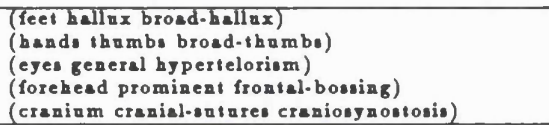 & \\
\hline N9 & 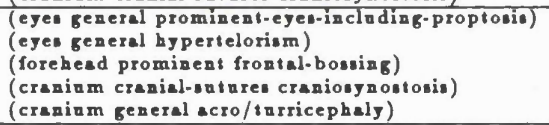 & \\
\hline
\end{tabular}

Table C.3: Node descriptions for Network R.2.

extent, with regard to the experimental results of the UNIMEM algorithm described in Chapter 4. The fact is that the overlap of features of the two cases that initially form a group provides the node description, and it is this node description that is updated with regard to later (matching) cases. Therefore, the initial node description formed by the first two overlapping cases determines to a great extent how the node evolves. A case presented later in the order which evaluates positively against the node may also have common features with one or more of the cases stored at that group (which were not included on the node description), but unless there is sufficient overlap to form a new sub-group, these features are not accounted for in the (parent) node description. A further ramification, therefore, is that the creation of sub-nodes can depend on the order of presented cases. This effect is demonstrated by Nodes N3 and N3.1 of Network R.1 as compared to Node N9 of Network R.2. The same five cases (four cases of Apert 


\begin{tabular}{|c|c|c|}
\hline Node & Permanent & Non-permanent \\
\hline N1 & & $\begin{array}{l}\text { (cranium cranial-outureb craniosynostosis) } \\
\text { (ejes general bypertelorism) } \\
\text { (face maxilla hypoplastic-maxilla-excl-malar-reg) } \\
\text { (earb ear-cras prominent-ear-cras) } \\
\text { (hands fingers skin-byndactyly-of-fingero) } \\
\text { (forehead prominent wide-forehead) }\end{array}$ \\
\hline N2 & & $\begin{array}{l}\text { (eye-siructures eyelido coloboms-of-ejelids) } \\
\text { (earo external-ears dyiplastic-ears) } \\
\text { (face malar-region fat-malar-region) } \\
\text { (eye-structures palpebral-fisurea } \\
\text { palpebral-fianres-slant-down) } \\
\text { (face mandible mall-mandible/micrognathia) }\end{array}$ \\
\hline N3 & & 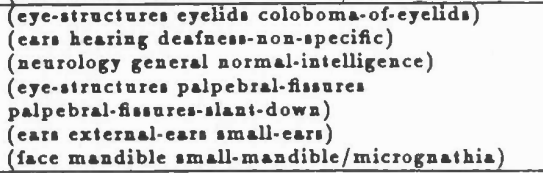 \\
\hline N4 & $\begin{array}{l}\text { (feet hallux broad-hallax) } \\
\text { (hando thumbs broad-thumbs) } \\
\text { (eyes general hypertelorism) } \\
\text { (forehead prominent frontal-bosing) } \\
\text { (craninm cranial-sutures craniosyostonis) }\end{array}$ & \\
\hline No & & 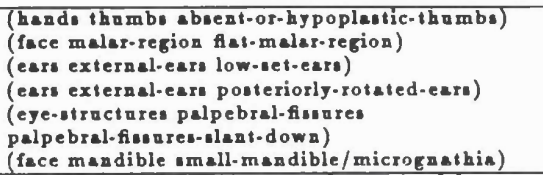 \\
\hline N6 & & $\begin{array}{l}\text { (craninm cranial-antaren crapiongnotonis) } \\
\text { (ejes general hypertelosiom) } \\
\text { (ears ear-crus prominent-ear-crus) }\end{array}$ \\
\hline N7 & & $\begin{array}{l}\text { (hands fingers absent-fingers-or-oligodactgly) } \\
\text { (feet toes absent-toes) } \\
\text { (oral-region palate clefi-palate) } \\
\text { (face malar-region fiat-malar-region) } \\
\text { (apper-limbs forearm bypoplastic-or-absent-nlna) } \\
\text { (face mandible amall-mandible/microgathia) }\end{array}$ \\
\hline N8 & 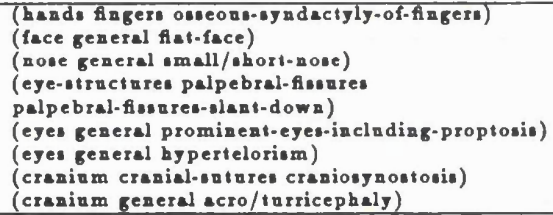 & \\
\hline No & $\begin{array}{l}\text { (feet loes absent-toes) } \\
\text { (hands fingers absent-fingera-or-oligodactyly) } \\
\text { (apper-limbs forearm hypoplastic-or-absent-alna) } \\
\text { (face mandible imall-mandible/microgasthis) }\end{array}$ & \\
\hline
\end{tabular}

Table C.4: Node descriptions for Network R.3.

syndrome and one case of Pfeiffer syndrome) are collectively grouped together in both these networks. However, whereas in Network R.1 a sub-node has been formed with Cases 564525 and 6761 (both Apert syndrome), in Network R.2 no such child node exists. Referring to Table C.1, it can be seen that N3 of Network R.1 is initially created by the overlap of Cases 0001 and 606067. In this instance, Cases 6761 and 564525 are the final two cases to join the group. Case 564525 is therefore stored with enough features that do not match Node N3 such that when Case 6761 evaluates with Node N3, its distinctive features overlap with Case 564525 (which is stored as an instance of Node N3) sufficiently to create a new child node. In Network R.2, Node N9 is initially constructed by the match between Case 6761 and Case 0001. Thus, the overlap between these two cases defines both the initial description of Node N9 and the list of distinctive 
features by which Case 6761 is stored (beneath Node N9). When Case 564525 evaluates with Node N9, its distinctive features do not provide sufficient overlap with Case 6761 in order to form a new sub-node.

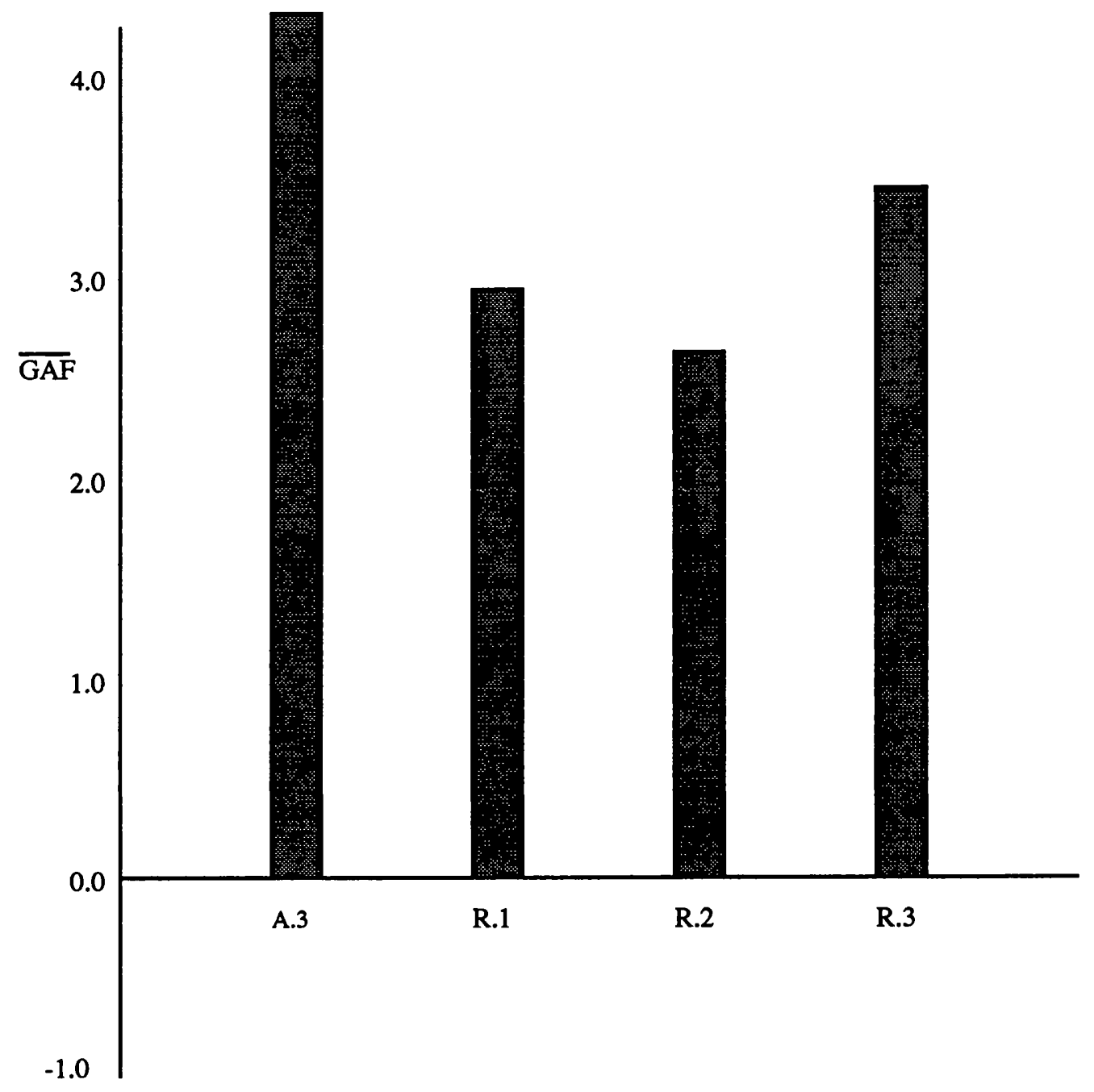

Figure C.4: Mean GAF for Networks A.3, R.1, R.2 and R.3

The acceptance of the assumption by Lebowitz, that over time (and with sufficient data) the effect of ordering is not strong, really depends on the importance attributed to the specific (qualitative) concept descriptions in terms of individual features. In terms of the general quantitative results (i.e., the mean group accuracy factors), the effect of ordering does not appear strong. However, when matching is performed merely with respect to two objects (either a case and node, or two cases) rather than in more general terms of the subject case, the node, and the cases stored at the node, then ordering will affect the formation of nodes and their associated descriptions. 


\section{Appendix D}

\section{Trace of Ranking and CBL}

\section{Procedure with Case $\mathbf{5 6 4 5 2 5}$}

TOP LEVEL MENU

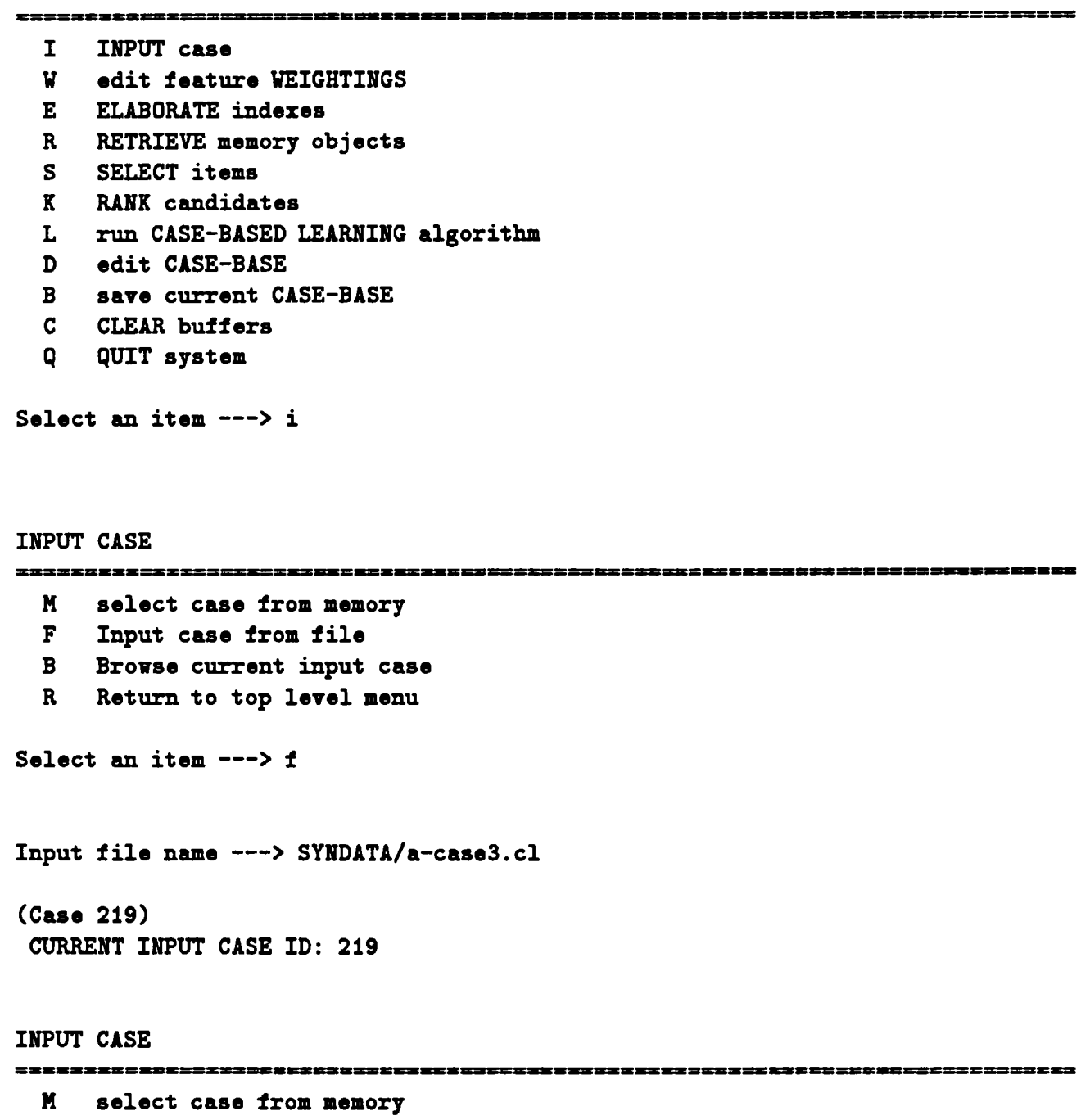




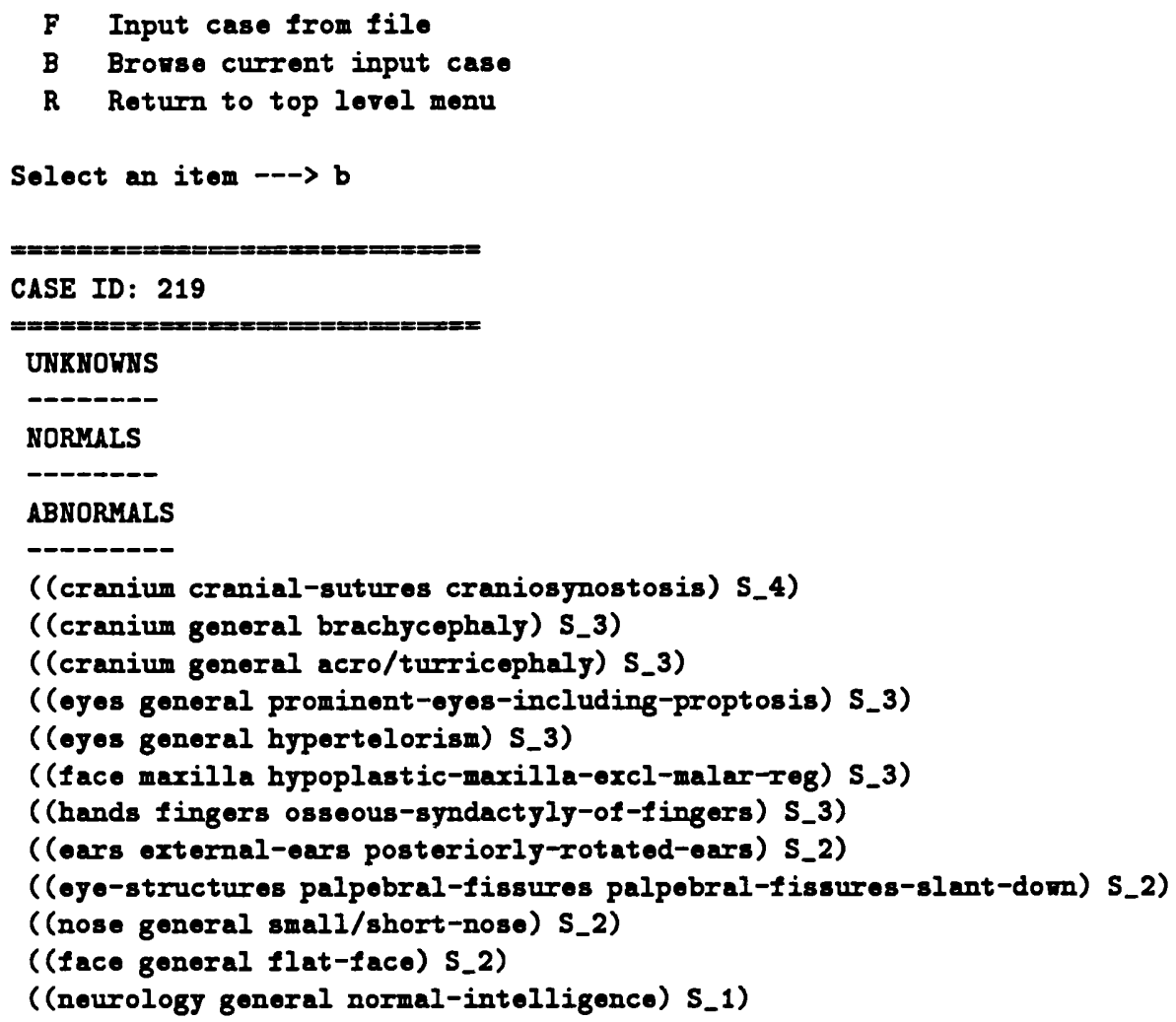




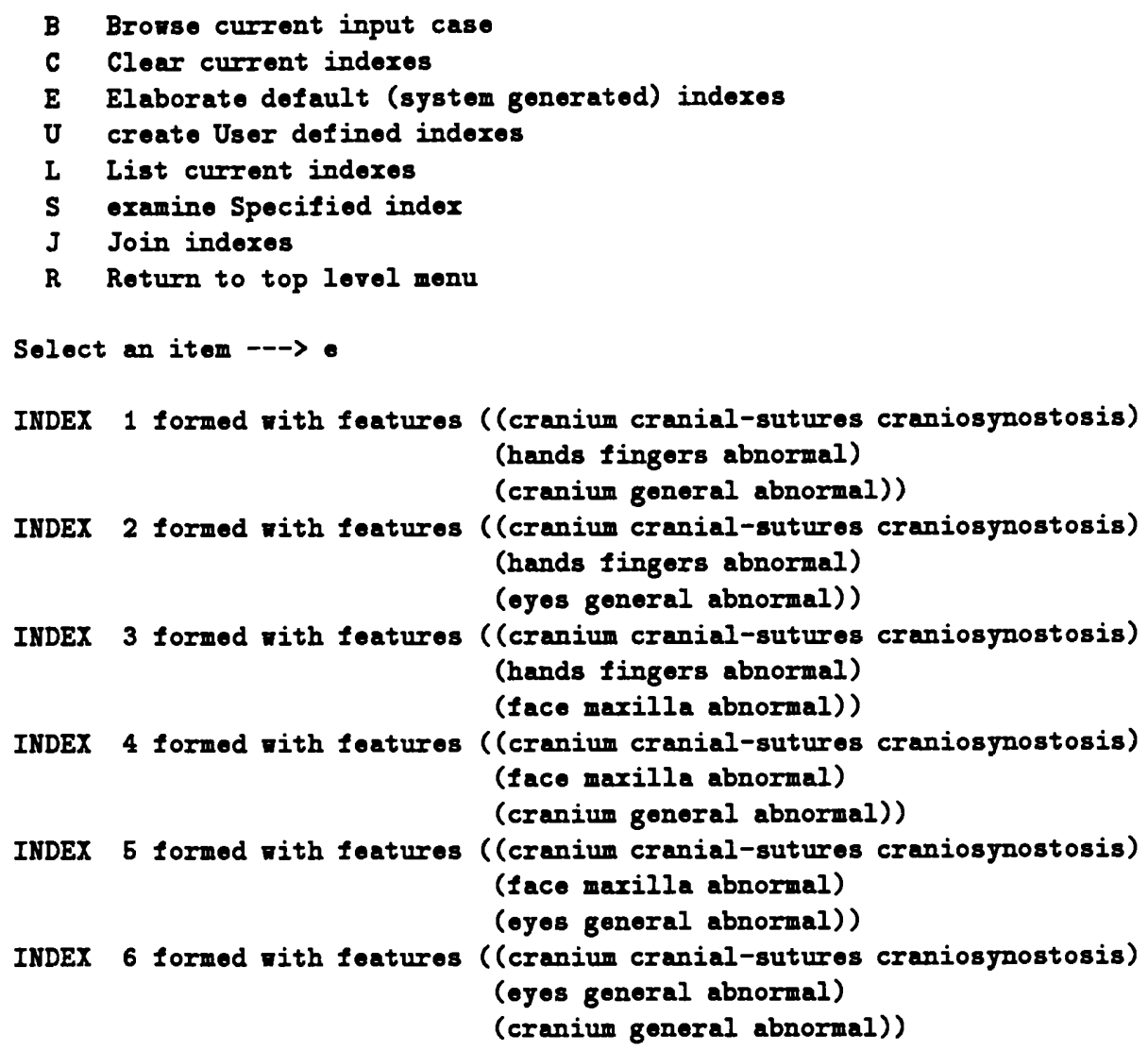


RETRIEVE (MATCH) MEMORY OBJECTS

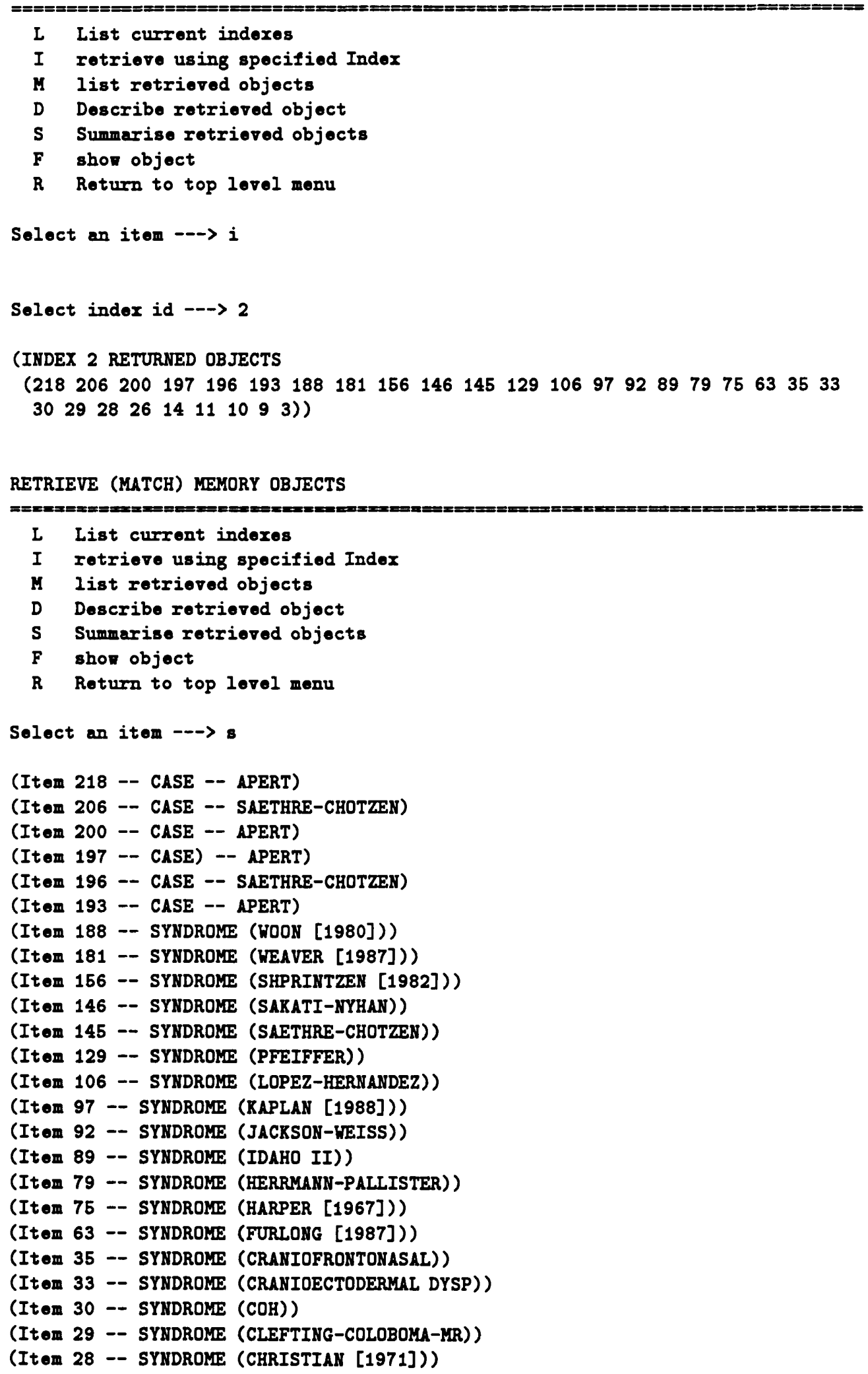


S select Subject

I select referent List

A rank with All syndromes

P Perform ranking with selected syndromes

$V$ Vien ranking 108

R Return to top level menu

Select an item $-->1$

Input selected objects --> (188 1811561461451291069792897975

$\left.\begin{array}{llllllllllll}63 & 35 & 33 & 30 & 29 & 28 & 26 & 14 & 11 & 10 & 9 & 3\end{array}\right)$

RANK Candidates

\begin{tabular}{|c|c|}
\hline $\boldsymbol{M}$ & Set parameters \\
\hline $\mathbf{S}$ & select Subject \\
\hline $\mathbf{L}$ & select referent List \\
\hline $\boldsymbol{A}$ & rank with All syndromes \\
\hline $\mathbf{P}$ & Perform ranking with selected syndromes \\
\hline $\mathbf{V}$ & Vien ranking $\log _{\mathrm{g}}$ \\
\hline $\mathbf{R}$ & Return to top level menu \\
\hline
\end{tabular}

Select an item $--\rightarrow p$

RANKED DIFFERENTIAL

(10 (APERT) 45.0)

(92 (JACKSON-WEISS) 32.0)

(145 (SAETHRE-CHOTZEN) 32.0 )

(156 (SHPRIHTZEN [1982]) 29.0)

(9 (ANYANE-YEBOA [1987]) 28.0)

(97 (KAPLAN [1988]) 28.0)

(188 (WOON [1980]) 28.0)

(35 (CRANIOFRONTONASAL) 24.0)

(129 (PFEIFFER) 24.0)

\section{RAIK Candidates}

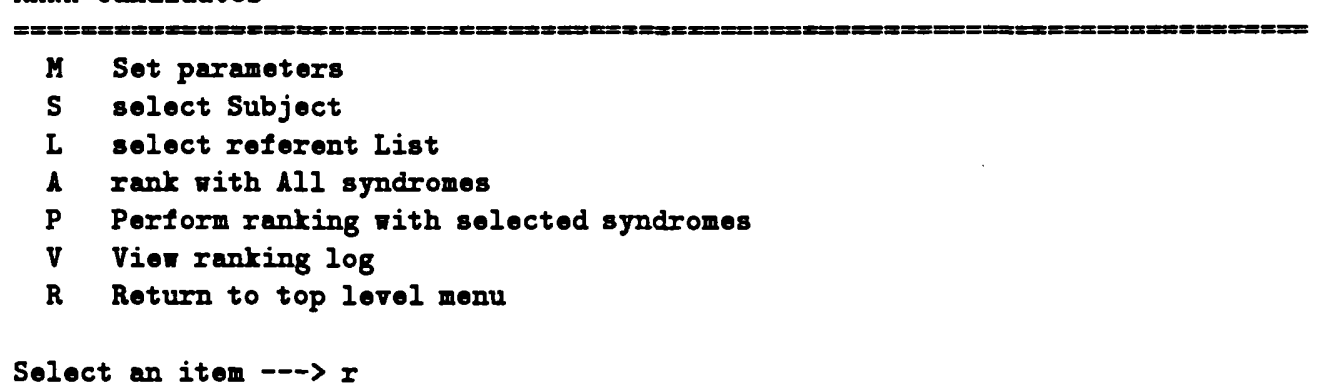

TOP LEVEL MENU

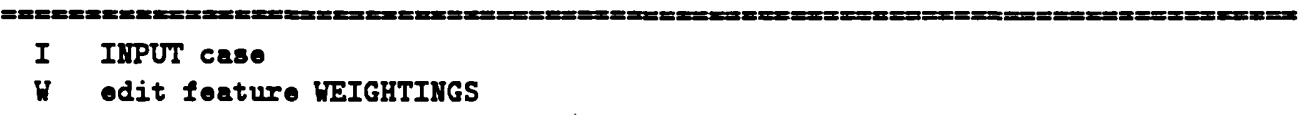


E ELABORATE inderes

R RETRIEVE memory objects

$S$ SELECT itoms

R RANK candidates

I run CASE-BASED LEARNING algorithm

D odit CASE-BASE

B savo curront CASE-BASE

C CLEAR buffers

Q QUIT system

Soloct an item ---> 1

Case-Based Loarning Model

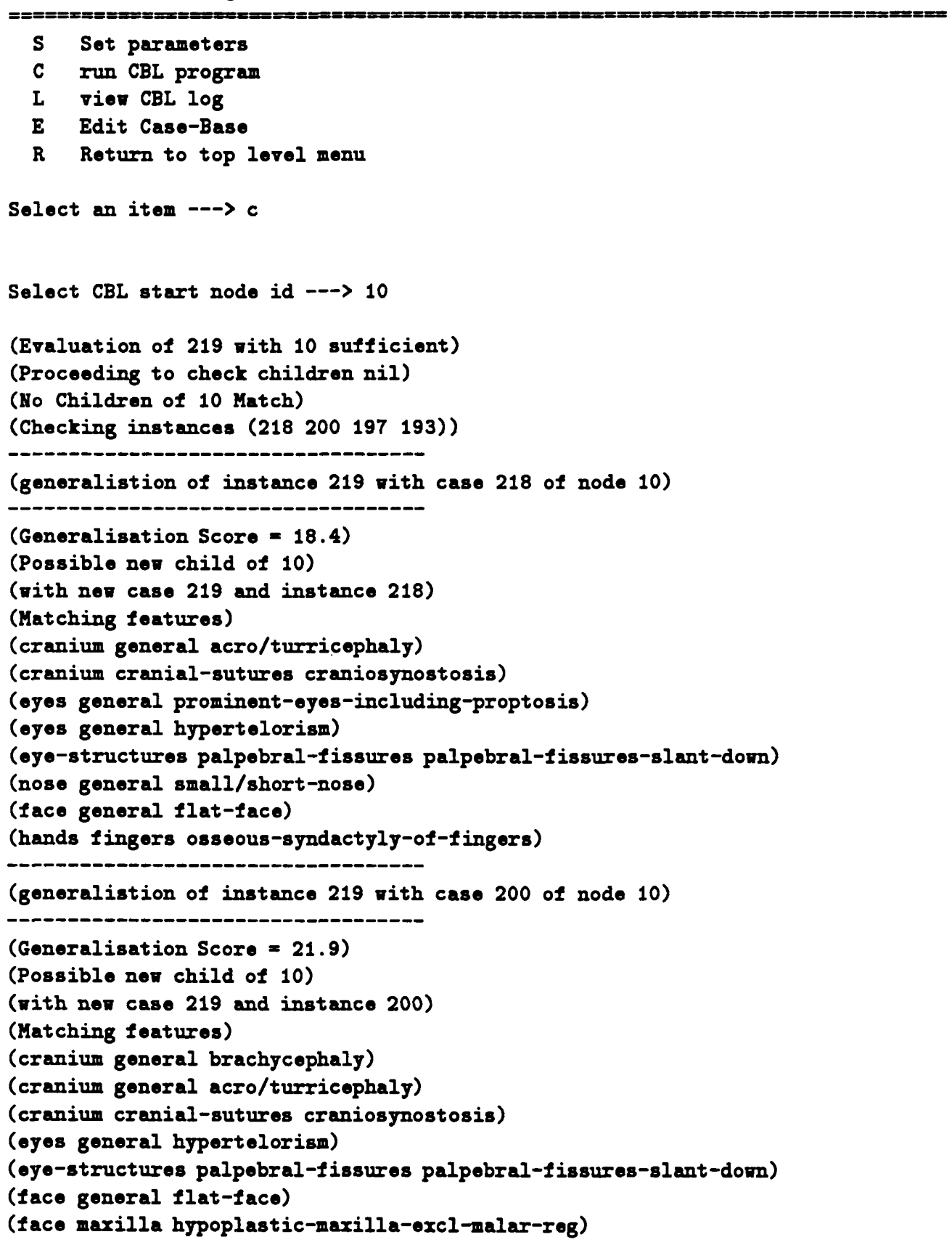


(hands fingers osseous-syndactyly-of-fingers)

(generalistion of instance 219 with case 197 of node 10)

(Generalisation Score $=27.1$ )

(Possible nor child of 10 )

(rith now case 219 and instance 197)

(Matching foatures)

(cranium general brachycephaly)

(cranium genoral acro/turricophaly)

(cranium cranial-sutures craniosynostosis)

(oyes general prominent-oyes-including-proptosis)

(oyes general hypertelorism)

(oye-structures palpebral-fissures palpobral-fissures-slant-domn)

(nose general small/short-nose)

(face genoral flat-face)

(face maxilla hypoplastic-maxilla-oxcl-malar-rog)

(hands fingers osseous-syndactyly-of-fingers)

(generalistion of instance 219 with case 193 of node 10)

(Goneralisation Score $=27.5$ )

(Possible ner child of 10 )

(with nor case 219 and instance 193)

(Matching features)

(cranium general brachycophaly)

(cranium general acro/turricophaly)

(cranium cranial-sutures craniosynostosis)

(oyes general prominent-eyes-including-proptosis)

(eyes general hypertelorism)

(ojo-structures palpobral-fissures palpobral-fissures-slant-domn)

(nose general small/short-nose)

(face goneral flat-face)

(face maxilla hypoplastic-maxilla-oxcl-malar-rog)

(hands fingers osseous-syndactyly-of-fingers)

(neurology goneral normal-intelligence)

Case-Based Loarning Model

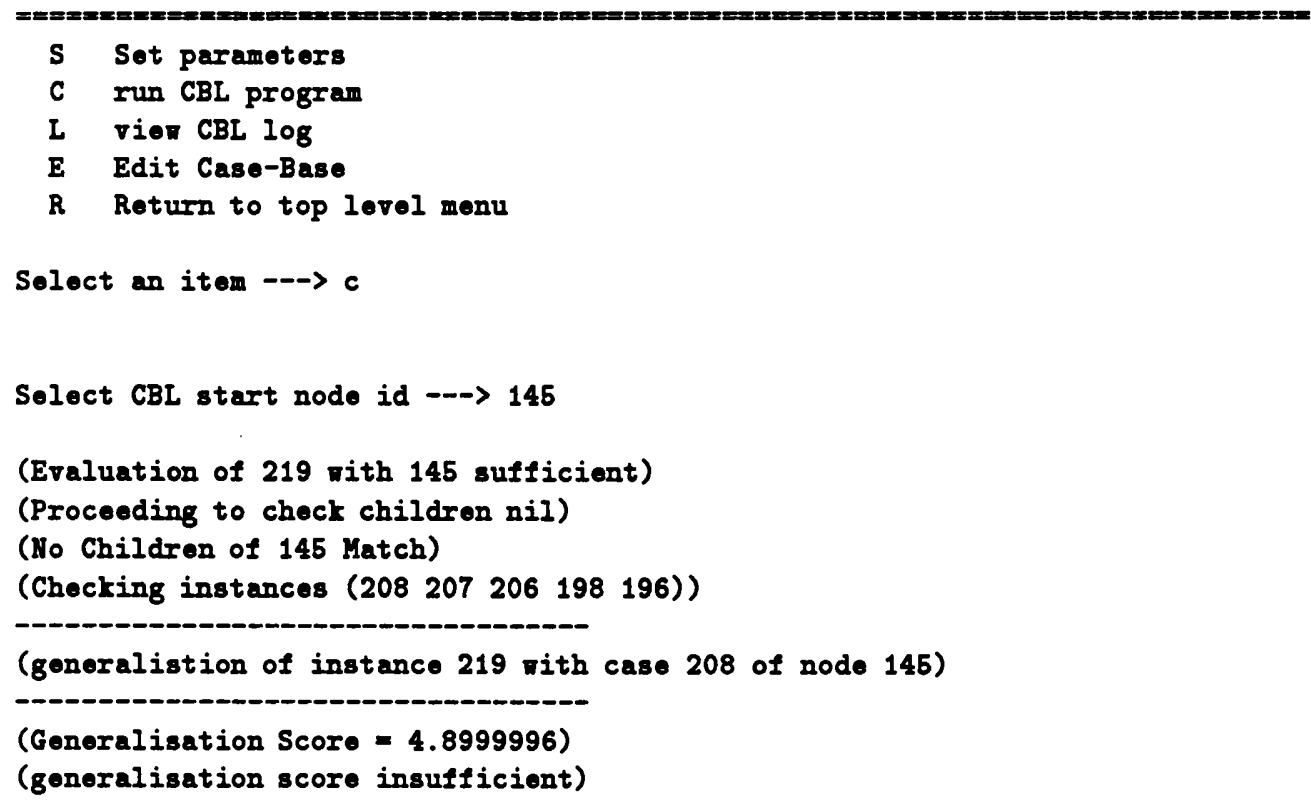


(generalistion of instance 219 with case 207 of node 145)

(Generalisation Score $=7.0999994)$

(Possible ner child of 145)

(rith nor case 219 and instance 207)

(Matching foaturos)

(cranium cranial-suturos craniosynostosis)

(oyes general hypertelorism)

(oye-structures palpebral-fissures palpebral-fissures-slant-domn)

(face maxilla hypoplastic-maxilla-oxcl-malar-rog)

-

(generalistion pf instance 219 vith case 206 of node 145)

(Generalisation Score $=4.7$ )

(generalisation score insufficient)

(generalistion of instance 219 with case 198 of node 145)

(Generalisation Score $=1.3000002$ )

(generalisation score insufficient)

(generalistion of instance 219 with case 196 of node 145)

(Generalisation Score $=9.799999)$

(Possiblo nor child of 145)

(rith ner case 219 and instance 196)

(Matching features)

(cranium goneral brachycophaly)

(cranium cranial-sutures craniosynostosis)

(oyos general hypertelorism)

(oyo-structures palpobral-fissures palpobral-fissures-slant-down)

(face maxilla hypoplastic-maxilla-excl-malar-rog)

Case-Based Loarning Model

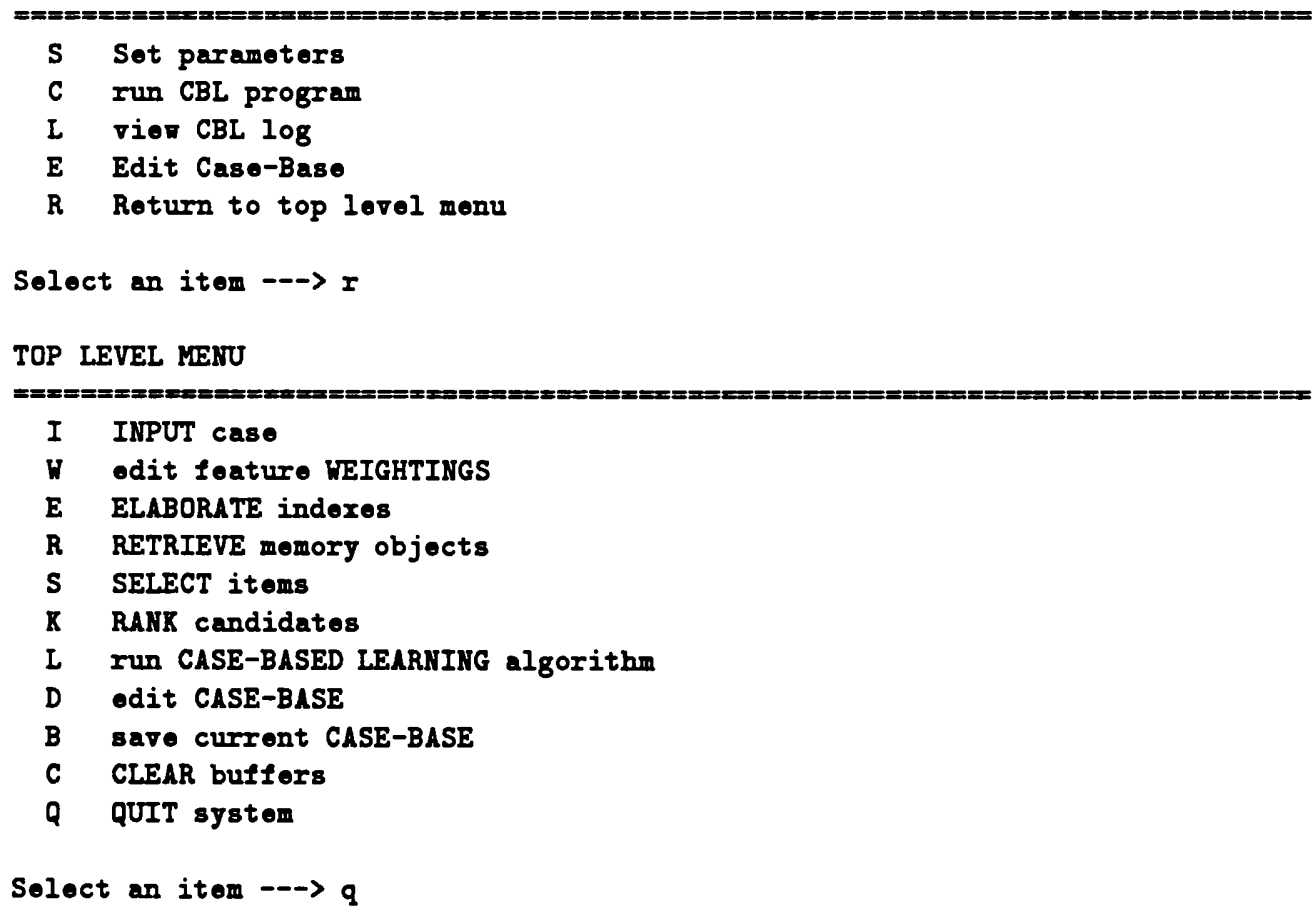




\section{Bibliography}

[1] Agnar Aamodt and Enric Plaza. Case-based reasoning: Foundational issues, methodological variations, and system approaches. AI Communications, 7(1):39$59,1994$.

[2] Richard Alterman. An adaptive planner. In $A A A I, 1986$.

[3] Richard Alterman. Adaptive planning. Cognitive Science, 12, 1988.

[4] Kevin D. Ashley. Arguing by analogy in law: A case-based model. In David H. Helman, editor, Analogical Reasoning, pages 205-224. Kluwer Academic Publishers, 1988.

[5] Kevin D. Ashley. Reasoning with cases and hypotheticals in HYPO. International Journal of Man-Machine Studies, 35(1), 1991.

[6] Agnes Bankier and C. Gregory Keith. POSSUM: The Microcomputer LaserVideodisk Syndrome Information System. Ophthalmic Paediatrics and Genetics, 10(1):51-52, 1989.

[7] Agnes Bankier and J. Marquet. Possum: A computer/laser videodisk system for syndrome diagnosis and learning about syndromes. The American Journal of Human Genetics Supplement, 49(4):28, 1991. Proceedings of the 8th International Congress of Human Genetics - Abstracts.

[8] Agnes Bankier, James J. McGill, Jennifer J. Danks, Jennifer A. McGill, and David M. Danks. Dysmorphology: Problems in Nomenclature. Dysmorphology and Clinical Genetics, 2:24-50, 1988.

[9] E.R. Bareiss. Exemplar-Based Knowledge Acquisition. Academic Press, New York, 1989.

[10] Ray Bareiss and James A. King. Similarity assessment in case-based reasoning. In Proceedings of the DARPA Case-Based Reasoning Workshop. Morgan Kaufmann, 1989.

[11] Jeff Berger. ROENTGEN a case-based approach to radiation therapy planning. In Proceedings of the DARPA Case-Based Reasoning Workshop. Morgan Kaufmann, 1989.

[12] G.W. Brown. Bayes' Formula. American Journal of Disease in Childhood, 135:1125$1129,1981$.

[13] M.L. Buyse. Center for Birth Defects Information Services. Birth Defects: Original Article Series, XVI(5):83-91, 1980. 
[14] M.L. Buyse and C.N. Edwards. The Birth Defects Information System. A computerbased information resource for diagnostic support, education, and research. American Journal of Perinatology, 4(1):8-11, 1987.

[15] R.M. Dawood, C.M. Hall, D. Shaw E.T. Keravnou, and J. Washbrook. A ModelBased Diagnostic Expert System for Skeletal Dysplasias. In J. Hunter, J. Cookson, and J. Wyatt, editors, AIME 89: Second European Conference on Artificial Intelligence in Medicine, pages 47-56. Springer-Verlag, 1989.

[16] John H. DiLiberti. Use of Computers in Dysmorphology. Journal of Medical Genetics, 25:445-453, 1988.

[17] B. S. Everitt. Statistical Methods for Medical Investigations. Oxford University Press, 1989.

[18] Douglas Fisher and Pat Langley. Approaches to conceptual clustering. In IJCAI, 1985.

[19] D.B. Flannery and S.L. Peterson. Practice analysis of dysmorphology diagnostic database. The American Journal of Human Genetics Supplement, 41:A59, 1987. Abstract.

[20] J.H. Gennari, P. Langley, and D. Fisher. Models of Incremental Concept Formation. Artificial Intelligence, 40(1-3), 1989.

[21] L. Gierl and S. Stengel-Rutkowski. Integrating consultation and semi-automatic knowledge acquisition in a prototype-based architecture: Experiences with dysmorphic syndromes. Artificial Intelligence in Medicine, 6(1):29-51, 1994.

[22] R.M. Goodman and R.J. Gorlin. The Malformed Infant and Child. Oxford University Press, 1983.

[23] J. Gouvernet, M. Caraboeuf, and S. Ayme. GENDIAG: A Computer-assisted Facility in Medical Genetics Based on Belief Functions. Methods of Information in Medicine, 24(4):177-180, 1985.

[24] K. Hammond and T. Converse. Panel discussion on case representation. In Proceedings of the DARPA Case-Based Reasoning Workshop. Morgan Kaufmann, 1989. Richard Alterman (Chair).

[25] Kristian J. Hammond. Chef: A model of case-based planning. In AAAI, 1986.

[26] Kristian J. Hammond. Case-based planning: A framework for planning from experience. Cognitive Science, 14(3), 1990.

[27] D. J. Hand. Discrimination and Classification. John Wiley \& Sons, 1986.

[28] Stephen Jose Hanson. Conceptual clustering and categorization: Bridging the gap between induction and causal models. In Ryszard S. Michalski and Yves Kodratoff, editors, Machine Learning: An Artificial Intelligence Approach, Volume III, pages 235-268. Morgan Kaufmann, 1990.

[29] Stephen Jose Hanson and Malcolm Bauer. Conceptual Clustering, Categorization and Polymorphy. Machine Learning, 3:343-372, 1989. 
[30] Kenneth Lyons Jones. Smith's Recognizable Patterns of Human Malformation. W. B. Saunders Company, fourth edition, 1988.

[31] Mark T. Keane. Analogical Problem Solving. Halsted Press, 1988.

[32] Sonya E. Keene. Object Oriented Programming with CLOS. Digital Press, 2nd edition, 1990.

[33] E.T. Keravnou and J. Washbrook. What is a deep expert system? An analysis of the architectural requirements of second-generation expert systems. The Knowledge Engineering Review, 4(3):205-233, 1989.

[34] E.T. Keravnou and J. Washbrook. A temporal reasoning framework used in the diagnosis of skeletal dysplasias. Artificial Intelligence in Medicine 2, pages 239-265, 1990.

[35] E.T. Keravnou, J. Washbrook, F. Dams, R.M. Dawood, C.M. Hall, and D. Shaw. Modelling Diagnostic Skills: Progress Report on the Skeletal Dysplasias Diagnostician. To be published.

[36] E.T. Keravnou, J. Washbrook, F. Dams, R.M. Dawood, C.M. Hall, and D. Shaw. A Model-Based Diagnostic Expert System for Diagnosis in Paediatric Skeletal Radiology. Radiology, 177:208, 1990.

[37] E.T. Keravnou, J. Washbrook, F. Dams, R.M. Dawood, C.M. Hall, and D. Shaw. Background knowledge in diagnosis. Artificial Intelligence in Medicine, 4(3):263279, 1992.

[38] Gregor Kiczales, Jim des Rivières, and Daniel G. Bobrow. The Art of the Metaobject Protocol. The MIT Press, 1st edition, 1991.

[39] Janet Kolodner, editor. Proceedings of the DARPA Case-Based Reasoning Workshop. Morgan Kaufmann, 1989.

[40] Janet Kolodner, editor. Proceedings of the DARPA Case-Based Reasoning Workshop. Morgan Kaufmann, 1991.

[41] Janet Kolodner. Case-Based Reasoning. Morgan Kaufmann Publishers, Inc., 1993.

[42] Janet L. Kolodner. Maintaining organisation in a dynamic long-term memory. Cognitive Science, 7, 1983.

[43] Janet L. Kolodner. Reconstructive memory: A computer model. Cognitive Science, $7,1983$.

[44] Janet L. Kolodner. Improving human decision making through case-based decision aiding. AI Magazine, pages 52-68, Summer 1991.

[45] Janet L. Kolodner and Robert M. Kolodner. Using experience in clinical problem solving: Introduction and framework. IEEE Transactions on Systems, Man, and Cybernetics, 17(3), 1987.

[46] Janet L. Kolodner and Robert L. Simpson. The mediator: Analysis of an early case-based problem solver. Cognitive Science, 13, 1989. 
[47] Phyllis Koton. Reasoning about evidence in causal explanations. In $A A A I, 1988$.

[48] Phyllis Koton. Reasoning about evidence in causal explanations. In Proceedings of the DARPA Case-Based Reasoning Workshop. Morgan Kaufmann, 1988.

[49] Michael Lebowitz. Categorizing numeric information for generalisation. Cognitive Science, 9(3):285-308, 1985.

[50] Michael Lebowitz. Concept Learning in a Rich Input Domain: GeneralizationBased Memory. In Machine Learning: An Artificial Intelligence Approach, Volume II, pages 193-214. Morgan Kaufmann, 1986.

[51] V. A. McKusick. Mendelian Inheritance in Man. Catalogs of Autosomal Dominant, Autosomal Recessive, and X-linked Phenotypes. Baltimore and London: The Johns Hopkins University Press, 8th edition, 1988.

[52] Ryszard S. Michalski and Robert E. Stepp. Learning from observation: Conceptual clustering. In Ryszard S. Michalski, Jaime G. Carbonnel, and Tom M. Mitchel, editors, Machine Learning: An Artificial Intelligence Approach, Volume I, pages 331-363. Morgan Kaufmann, 1983.

[53] Ryszard S. Michalski and Robert E. Stepp. Conceptual clustering: Inventing goaloriented classifications of structured objects. In Machine Learning: An Artificial Intelligence Approach, Volume II, pages 471-498. Morgan Kaufmann, 1986.

[54] Oxford Electronic Publishing, Oxford University Press. London Dysmorphology Database. Marketing Information Sheet.

[55] Michael A. Patton. A Computerized Approach to Dysmorphology. M.D. Computing, 4(2), 1987.

[56] Bruce W. Porter, Ray Bareiss, and Robert C. Holte. Concept learning and heuristic classification in weak theory domains. Artificial Intelligence, 45, 1990.

[57] Marilyn Preus. The Numerical Verses Intuitive Approach to Syndrome Nosology. Birth Defects: Original Article Series, XVI(5):93-104, 1980.

[58] Marilyn Preus and Sègoléne Aymé. Formal analysis of dysmorphism: Objective methods of syndrome definition. Clinical Genetics, 23:1-16, 1983.

[59] J. R. Quinlan. Induction of decision trees. Machine Learning, 1:81-106, 1986.

[60] Christopher K. Riesbeck and Roger C. Schank. Inside Case-Based Reasoning. Lawrence Erlbaum Associates, 1989.

[61] D.L. Rimoin, October 1992. Personal communication.

[62] Richard Roiger. An exemplar-based approach to concept learning. AISB Quarterly, Special Issue on AI in Medicine, Winter 1992.

[63] E. Rosch and C.B. Mervis. Family Resemblance: Studies in the Internal Structures of Categories. Cognitive Psychology, 7:573-605, 1975.

[64] L.J. Salgado, J.S Lopez-Camelo, and E.E. Castilla. EL BUSCA and the value of signals in the diagnosis of dysmorphic syndromes: good and bad handles in computer assisted differential diagnosis. Journal of Medical Genetics, 27:446-450, 1990. 
[65] Roger C. Schank. Dynamic Memory. Cambridge University Press, 1982.

[66] Daniel F. Schorderet. Diagnosing Human Malformation Patterns With a Microcomputer: Evaluation of Two Different Algorithms. American Journal of Medical Genetics, 28:337-344, 1987.

[67] Daniel F. Schorderet. Using OMIM as an Expert System in Medical Genetics. American Journal of Medical Genetics, 39:278-284, 1991.

[68] Daniel F. Schorderet and P. Aebischer. SYNDROC: microcomputer based differential diagnosis of malformation patterns. Archives of Disease in Children, 60:248-251, 1985.

[69] J. Spranger. International classification of ostechondrodysplasias. European Journal of Pediatrics, 151:407-415, 1992.

[70] J. Spranger, K. Benirschke, J.G. Hall, W. Lenz, R.B. Lowry, J.M. Opitz, L. Pinsky, H.G. Schwarzacher, and D.W. Smith. Errors of morphogenesis: Concepts and terms. The Journal of Pediatrics, 100(1):160-165, 1982.

[71] Guy L. Steele. Common Lisp. Digital Press, 3rd edition, 1989.

[72] P. Stromme. The Diagnosis of Syndromes by Use of a Dysmorphology Database. Acta Paediatr. Scand., 80:106-109, 1991.

[73] Katia Sycara. Resolving goal conflicts via negotiation. In $A A A I, 1988$.

[74] Katia Sycara. Using case-based reasoning for plan adaptation and repair. In Proceedings of the DARPA Case-Based Reasoning Workshop. Morgan Kaufmann, 1988.

[75] Hooshang Taybi and Ralph S. Lachman. Radiology of Syndromes, Metabolic Disorders, and Skeletal Dysplasias, 3rd Edition. Year Book Medical Publishers, Inc, 1990.

[76] Roy M. Turner. Organizing and using schematic knowledge for medical diagnosis. In Proceedings of the DARPA Case-Based Reasoning Workshop. Morgan Kaufmann, 1988.

[77] Amos Tversky. Features of similarity. Psychological Review, 84(4):327-352, 1977.

[78] Amos Tversky. Studies of similarity. In Eleanor Rosch and Barbara B. Lloyd, editors, Cognition and Categorization, chapter 4, pages 79-98. Lawrence Erlbaum Associates, 1978.

[79] M.L. Veloso and M.J. Feijóo. DYSMOR: Computer Based Differential Diagnosis of Dysmorphic Syndromes. In R. Salamon, B. Blum, and M. Jorgensen, editors, Proceedings of Medinfo 1986, pages 216-218. Elsevier Science Publishers, 1986.

[80] M.L. Veloso and M.J. Feijóo. Microcomputer based differential diagnosis of dysmorphic syndromes. Ophthalmic Paediatrics and Genetics, 10(1):47-50, 1989.

[81] Fred Weiner and Göran Annerén. Pc-based system for classifying dysmorphic syndromes in children. Computer Methods and Programs in Biomedicine, 28:111-117, 1989. 
[82] Fred Weiner, Merav Gabbai, and Michael Jaffe. Computerized classification of congenital malformations using a modified bayesian approach. Comput. Biol. Med., 17(4):259-267, 1987.

[83] Patrick Henry Winston and Berthold Klaus Paul Horn. Lisp. Addison-Wesley, 2nd edition, 1990.

[84] R. M. Winter and M. Baraitser. Malformation syndromes - a diagnostic approach. Archives of Diseases in Childhood, 59:294-295, 1984.

[85] R. M. Winter and M. Baraitser. Multiple Congenital Anomalies. Chapman and Hall Medical, 1991.

[86] R. M. Winter, M. Baraitser, and J. M. Douglas. A computerised data base for the diagnosis of rare dysmorphic syndromes. Journal of Medical Genetics, 21:121-123, 1984.

[87] R. M. Winter, R. D Clark, K. Ashley, and G. Gibbs. A combinatorial method for grouping cases with multiple malformations. Journal of Medical Genetics, 25:118$121,1988$.

[88] R.M. Winter, December 1993. Personal communication.

[89] R.M. Winter and M. Baraitser. Syndrome Program. Technical Report and User Guide, 1986.

[90] K. Yamamoto and M. Sudo. A design of medical consultation system using standard MUMPS. In Proceedings of 4th JCMI, pages 580-583, 1984.

[91] K. Yamamoto, M. Sudo, Y. Shigematsu, M. Fukui, and J. Masukawa. The development of personal computer-based medical consultation system for diagnosis of congenital malformation syndromes using MUMPS. Medical Informatics, 15:355$362,1990$.

[92] The American Journal of Human Genetics. The University of Chicago Press.

[93] Clinical Dysmorphology. Chapman \& Hall, London.

[94] Human Genetics. Springer-Verlag, Berlin.

[95] Journal of Medical Genetics. BMJ Publishing Group, London.

[96] American Journal of Medical Genetics. Wiley-Liss, Inc. New York.

[97] Proceedings of the DARPA Case-Based Reasoning Workshop. Morgan Kaufmann, 1988.

[98] First European Workshop on Case-Based Reasoning (EWCBR '93). Springer-Verlag, 1993. 